\begin{tabular}{|c|c|c|c|}
\hline \multirow{2}{*}{\multicolumn{2}{|c|}{ JUL 1 1997\% ENGINEERING DATA TRANSMITTAL }} & \multicolumn{2}{|c|}{ Page 1 of 1} \\
\hline & & 1. EDT & 601047 \\
\hline
\end{tabular}

2. To: (Receiving organization) Distribution

5. Proj./Prog./Dept./Div.:

N4H2B/Characterization

Equipment Design

8. Originator Remarks:

This Test Report is submitted for review and approval.

Engineering Testing

Laboratory (8C921)

6. Design Authority/ Design Agent/Cog. Engr::

E.J. WALDO
3. From: (originating Organization)

4. Related EDT No.:

618744

7. Purchase Order No.:

$$
\text { N/A }
$$

9. Equip./Component No.:

$\mathrm{N} / \mathrm{A}$

10. System/Bldg./Facility:

$N / A$

11. Receiver Remarks: 11A. Design Basel ine Document?

12. Major Assm. Dwg. No.:

$N / A$
13. Permit/Permit Application No.: $\mathrm{N} / \mathrm{A}$

14. Required Response Date: ASAP

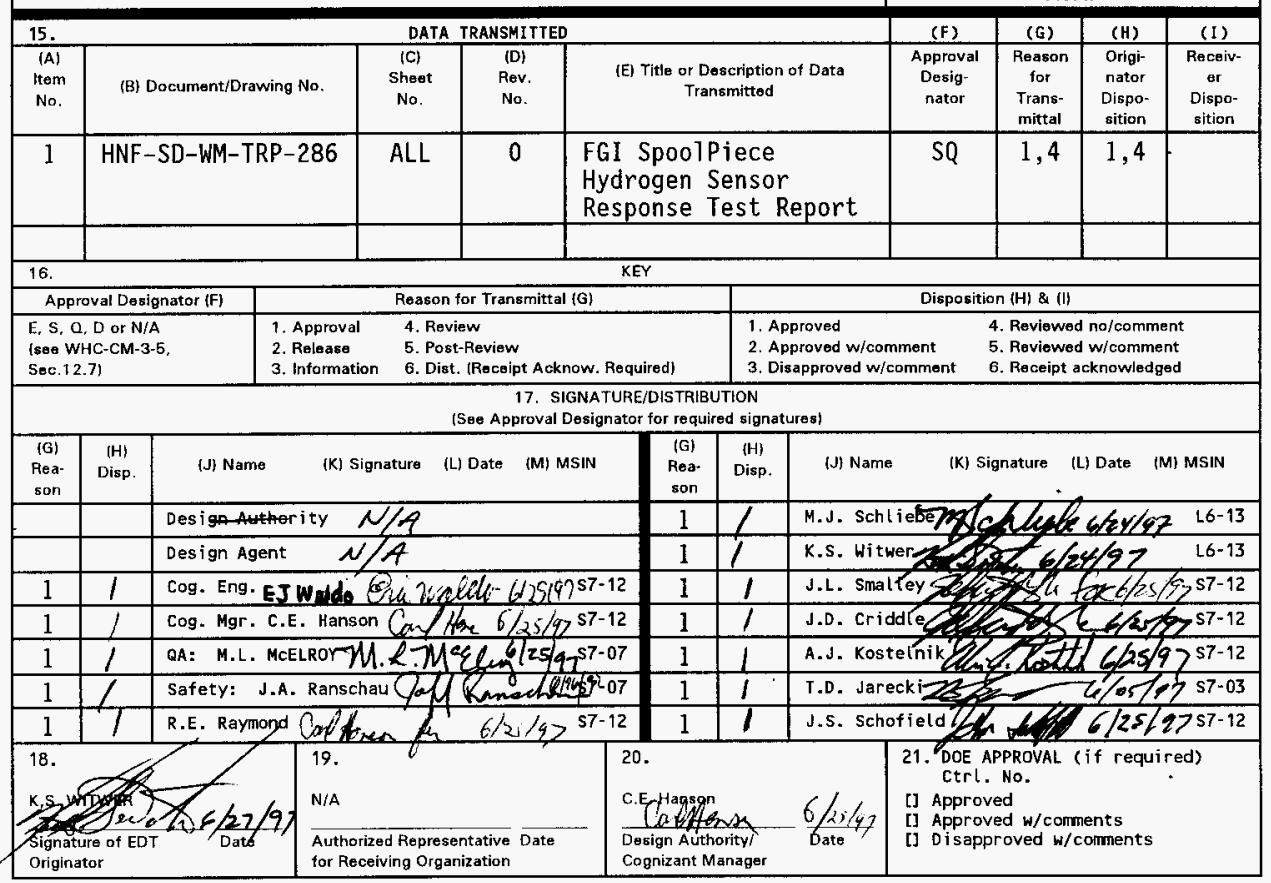




\section{FGI SPOOLPIECE HYDROGEN SENSOR RESPONSE TEST REPORT}

Keith S. Witwer

Engineering Testing Laboratory, Numatec Hanford Co., Richland, WA 99352 U.S. Department of Energy Contract DE-AC06-96RL13200

EDT/ECN: 601047

Org Code: $8 \mathrm{C} 921$

B\&R Code: EW3120072
UC: 2030

Charge Code: $\mathrm{N} 4 \mathrm{H} 2 \mathrm{~B}$

Total Pages: 263

Key Words: FGI, SpoolPiece, SMC, Detronics, ETL, Whittaker, Flammable Gas, Hydrogen, Exhauster

Abstract: This report documents the results of testing of three flammable gas sensors used on the Rotary Mode Core Sampling System (RMCSS) Flammable Gas Interlock (FGI). These sensors, a Detector Electronics Inc. (Detronics), a Sierra Monitor Corp. (SMC), and a Whittaker Electronics Corp. (WEC) can detect flammable gases and terminate the core sampling activity at a predetermined gas

concentration. Testing showed that each of the units can adequately detect a hydrogen gas mixture within a specified time frame and be accurate within manufacturers specifications.

TRADEMARK DISCLAIMER. Reference herein to any specific cormercial product, process, or service by trade name, trademark, manufacturer, or otherwise, does not necessarily constitute or imply its endorsement, recommendation, or favoring by the United States Government or any agency thereof or $i$ ts contractors or subcontractors.

Printed in the United states of America. To obtain copies of this document, contact: Document Control Services, P.0. Box 950, Mailstop H6-08, Richland WA 99352, Phone (509) 372-2420; Fax (509) 376-4989.
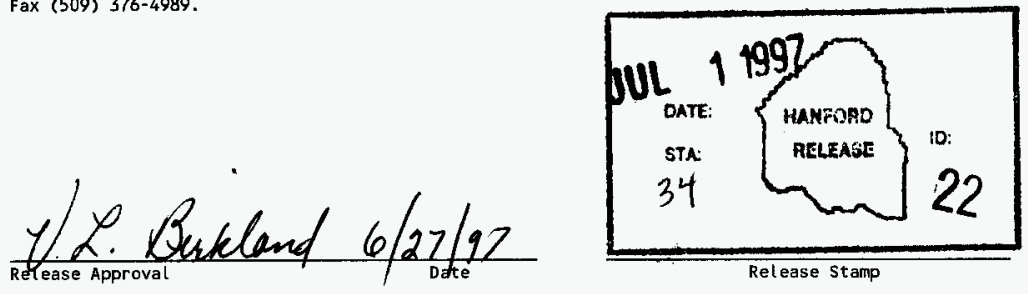

Release Stamp 


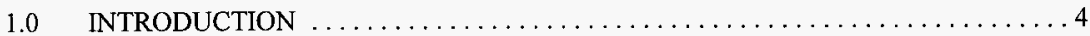

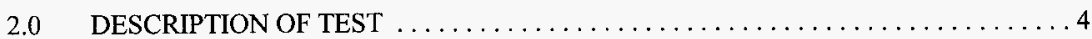

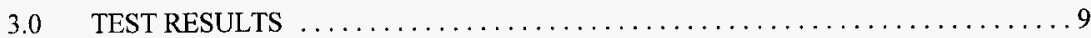

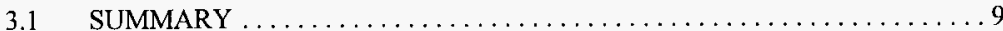

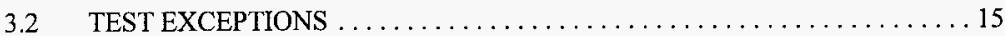

4.0 CONCLUSIONS AND RECOMMENDATIONS $\ldots \ldots \ldots \ldots \ldots \ldots \ldots \ldots \ldots, \ldots \ldots$

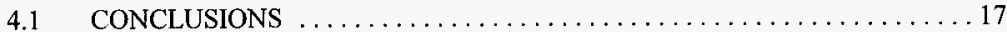

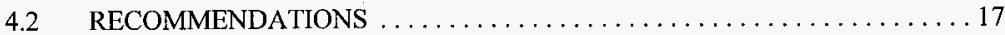

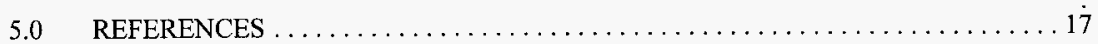

APPENDIX A - MEASUREMENT AND TEST EQUIPMENT DATA SHEET $\ldots \ldots \ldots \ldots 18$

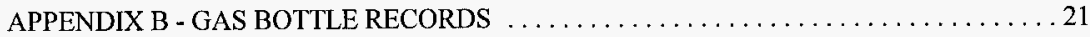

APPENDIX C - MANUALLY ENTERED DATA SHEETS

AND SENSOR OUTPUT GRAPHS $\ldots \ldots \ldots \ldots \ldots \ldots \ldots \ldots 23$

APPENDIX D - EXCEPTION RECORDS $\ldots \ldots \ldots \ldots \ldots \ldots \ldots \ldots \ldots \ldots \ldots \ldots \ldots \ldots \ldots \ldots \ldots$

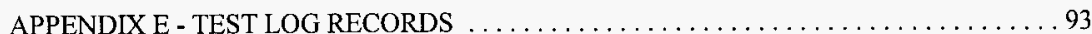

APPENDIX F - DETRONICS FLAMMABLE GAS SENSOR STARTUP AND

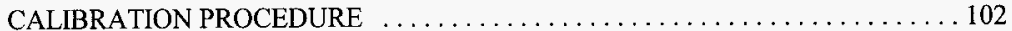

APPENDIX G - DATA ACQUISITION SYSTEM SPREADSHEET FILES $\ldots \ldots \ldots \ldots \ldots$ 


\section{LIST OF PHOTOS}

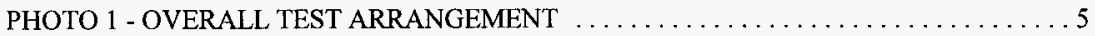

PHOTO 2 - WHITTAKER SENSOR MOUNTING $\ldots \ldots \ldots \ldots \ldots \ldots \ldots \ldots \ldots \ldots \ldots \ldots \ldots \ldots \ldots$

PHOTO 3 - DETRONICS SENSOR MOUNT $\ldots \ldots \ldots \ldots \ldots \ldots \ldots \ldots \ldots \ldots \ldots \ldots \ldots \ldots \ldots \ldots$

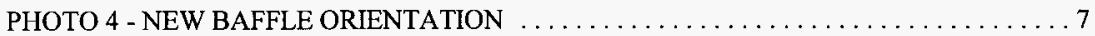

\section{LIST OF FIGURES}

FIGURE 1 - TEST CONFIGURATION P\&ID $\ldots \ldots \ldots \ldots \ldots \ldots \ldots \ldots \ldots \ldots$

\section{LIST OF TABLES}

TABLE 1 - CONSOLIDATED TEST RESULTS

USING SMC AND WHITTAKER SENSORS $\ldots \ldots \ldots \ldots \ldots \ldots \ldots, 10$

TABLE 2 - CONSOLIDATED TEST RESULTS

USING DETRONICS AND WHITTAKER SENSORS $\ldots \ldots \ldots \ldots \ldots \ldots 12$

TABLE 3 - SENSOR RESPONSE COMPARISON

SHOWING TIME TO REACH 90\% OF FINAL (15 MINUTE) VALUE $\ldots \ldots 14$ 
HNF-SD-WM-TRP-286

Rev. 0

Page 4

\section{$1.0 \quad$ INTRODUCTION}

The purpose of this test report is to document the testing performed under the guidance of Test Plan HNF-SD-WM-TC-074, Flammable Gas Sensors and SpoolPiece Flow Response Test Plan and Procedure ${ }^{I}$. This testing was performed for Lockheed Martin Hanford Characterization Projects Operations (CPO) in support of Rotary Mode Core Sampling by the Numatec Hanford Company Engineering Testing Laboratory (ETL). The testing was conducted in the 305 Building, ETL facility. The key personnel identified for the performance of this task are as follows:

- Test responsible engineering manager: C. E. Hanson

- 305 ETL responsible manager: M. J. Schliebe

- Cognizant RMCSS Exhauster engineer: E. J. Waldo

- Cognizant 305 ETL engineer: K. S. Witwer

Other support personnel were provided, as necessary, from the $305 \mathrm{ETL}$.

The testing was done on the flammable Gas Interlock (FGI) system Spool-Piece, which is required to support Rotary Mode Core Sampling (RMCSS) of single shell flammable gas watch list tanks. It took place between 5-5-97 and 5-23-97.

\subsection{DESCRIPTION OF TEST}

There were two primary purposes for this testing. One was to verify the gas delivery characteristics of the mechanical SpoolPiece system by determining typical sensor response times as installed in their deployed configuration. Earlier testing by Schneider ${ }^{2}$ showed the adequacy of the existing system. A change to the SpoolPiece baffle however required the earlier testing be repeated. Secondly, the existing Sierra Monitor Corporation (SMC) catalytic bead hydrogen sensor has since been identified as not rated for a Class 1, Division 1, Group B environment by the NFPA. Another vendor, Detector Electronics Corporation, has a suitably rated sensor (Detronics) of a similar type. The Detronics sensor was installed on the SpoolPiece and also tested.

The main components of the test setup included metallic and flexible conductive ducting, a blower and flow control valve capable of providing the required duct flow, an accurate flow measuring system, an accurate hydrogen injection gas system, a mechanically equivalent FGI SpoolPiece with sensors, a gas chromatograph (GC) system to validate the duct gas concentrations, a data logger and associated process temperature and pressure instruments. Photo 1 shows an overall view of the test setup. The piping and instrumentation diagram (P\&ID) of Figure 1 depicts the test hardware configuration. A listing of the measurement and test equipment used and pertinent calibration records are included in Appendix A. 


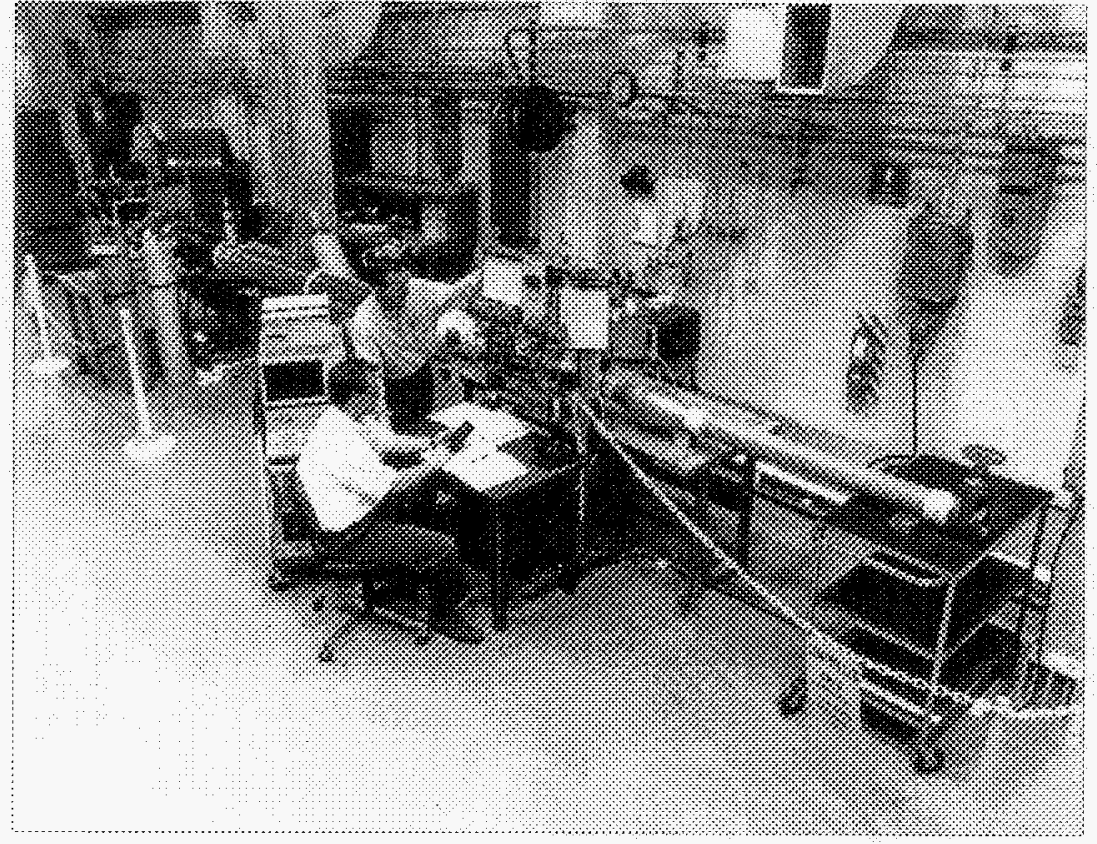

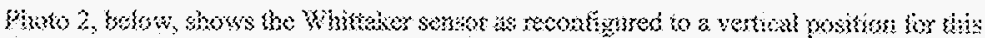

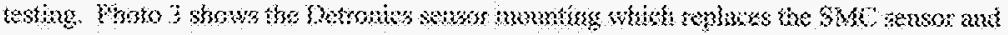

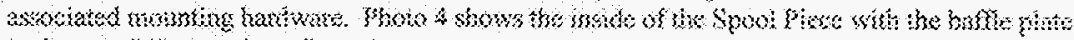

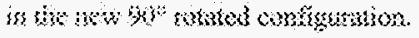




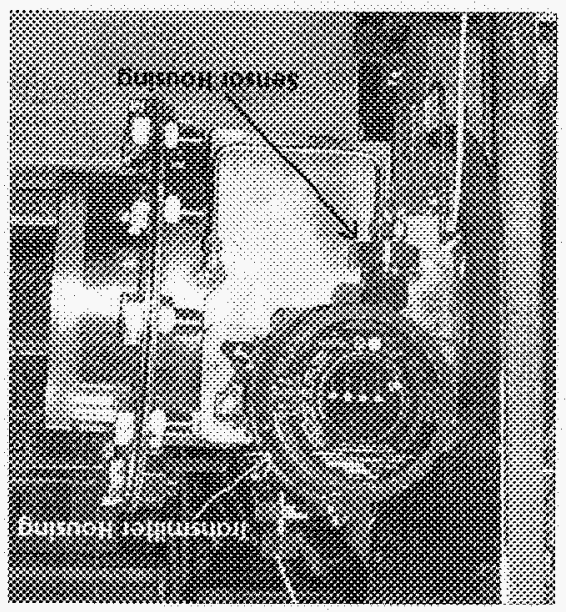

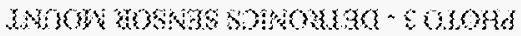

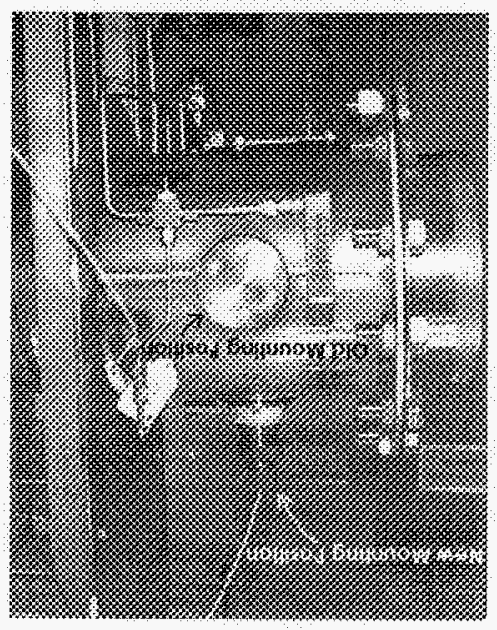

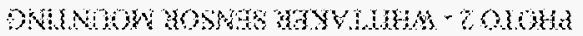

9 sing

a mas

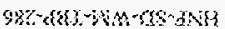




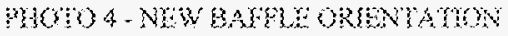

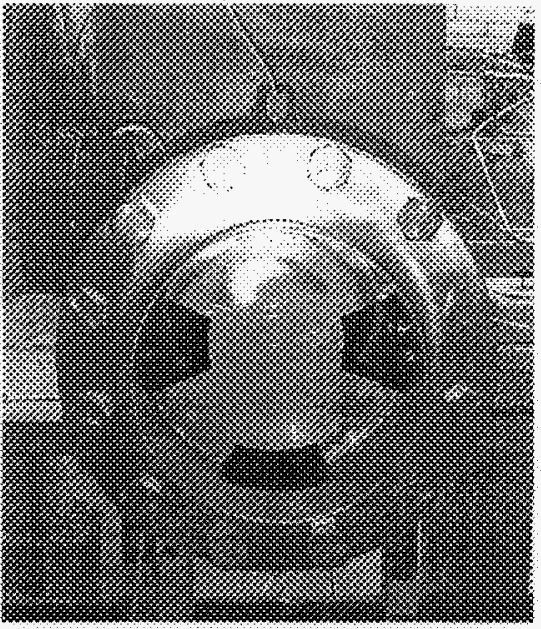

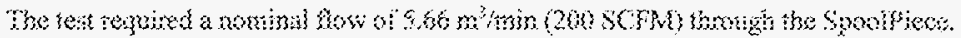

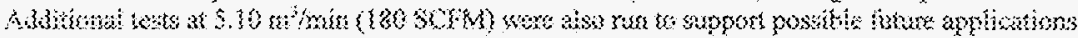

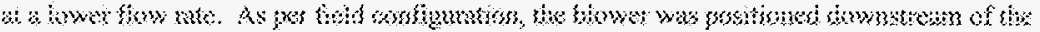

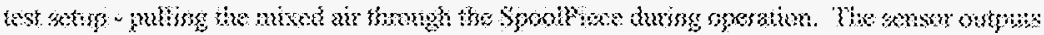

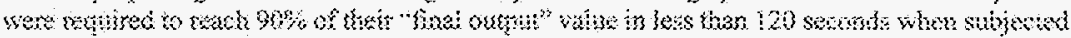

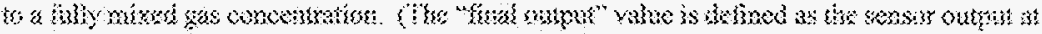

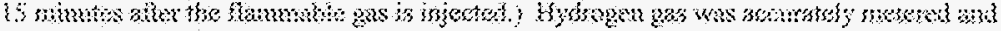

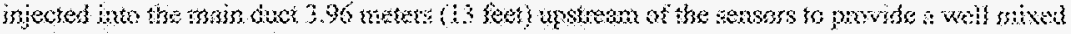

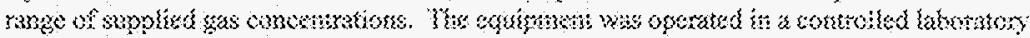

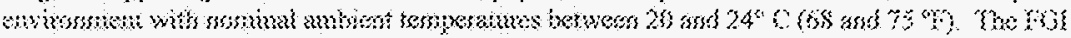

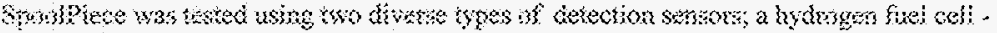

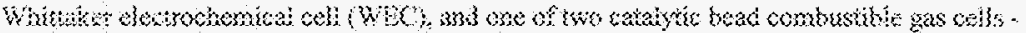

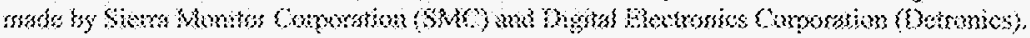




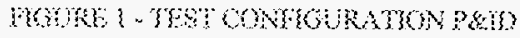

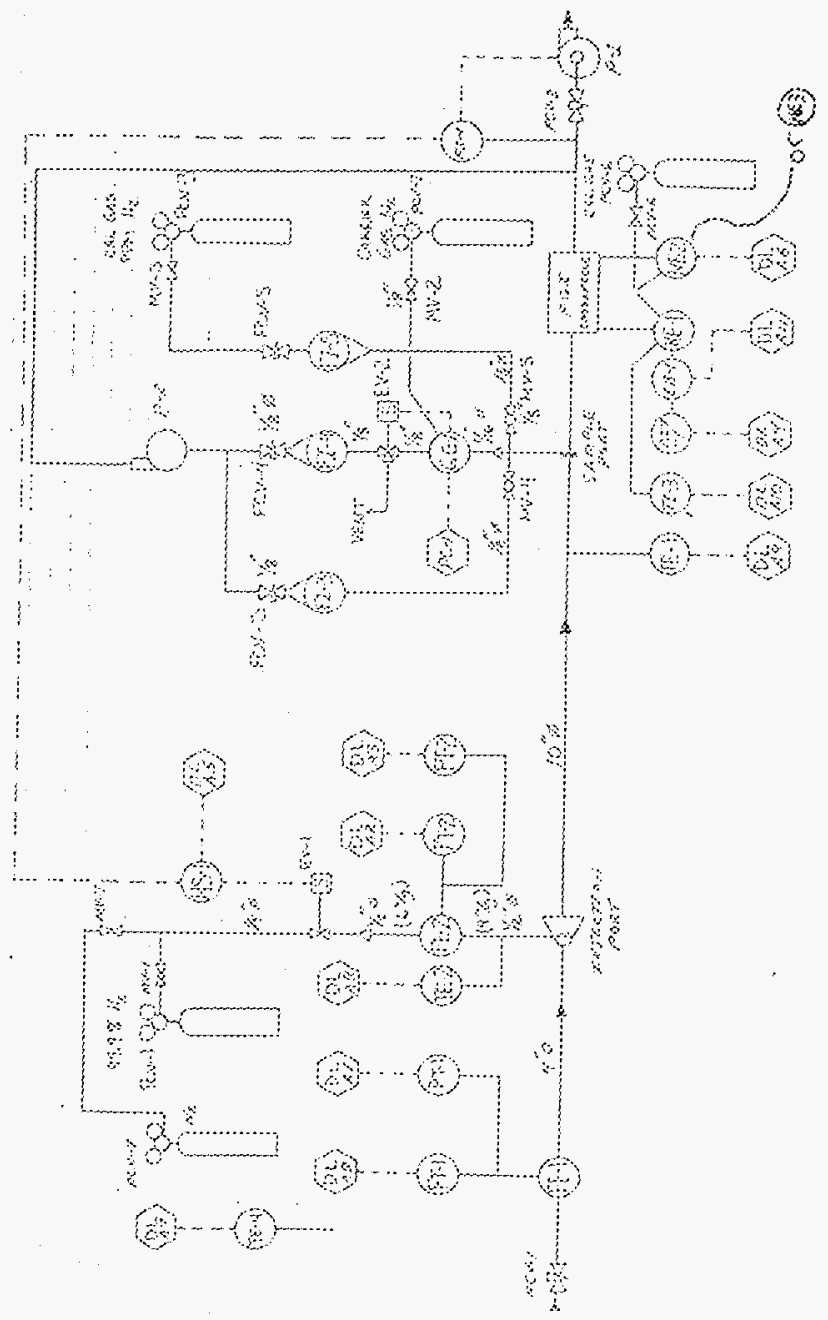




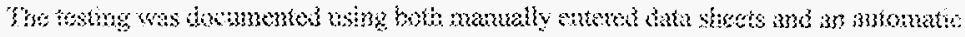

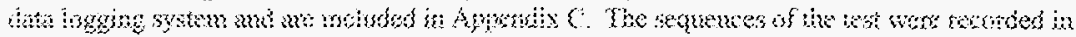

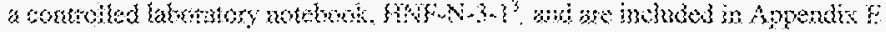

\section{0 meressing}

\section{3.: Sunthary}

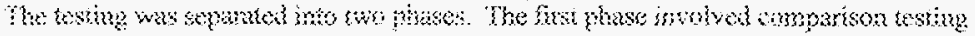

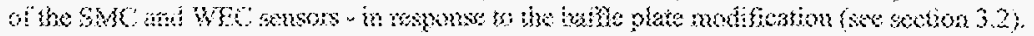

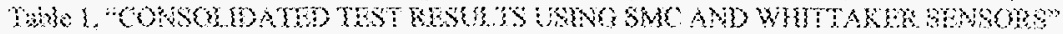

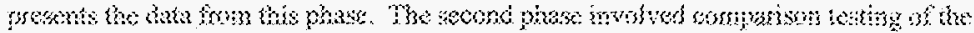

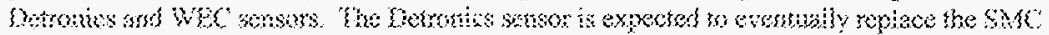

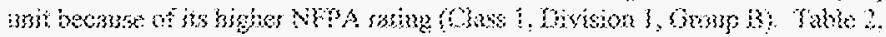

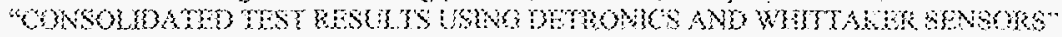

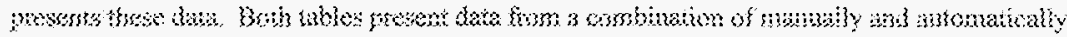

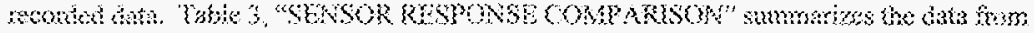
bot finges for comprom purposs.

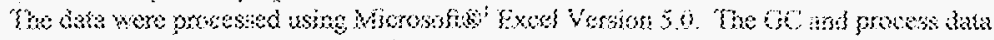

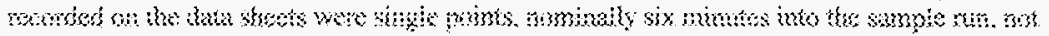

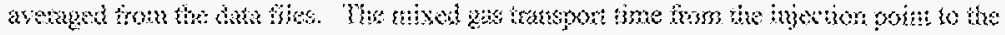

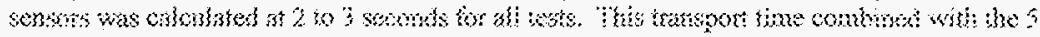

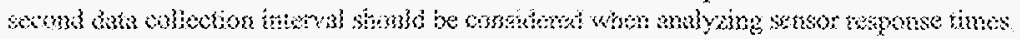

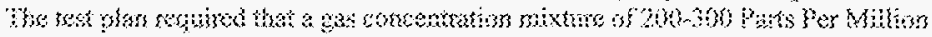

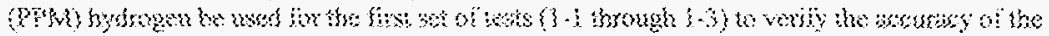

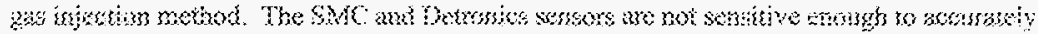

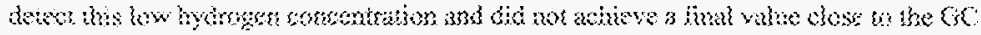

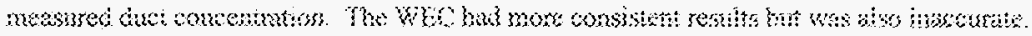

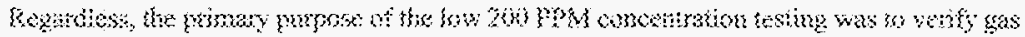

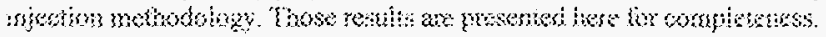

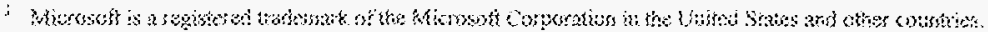




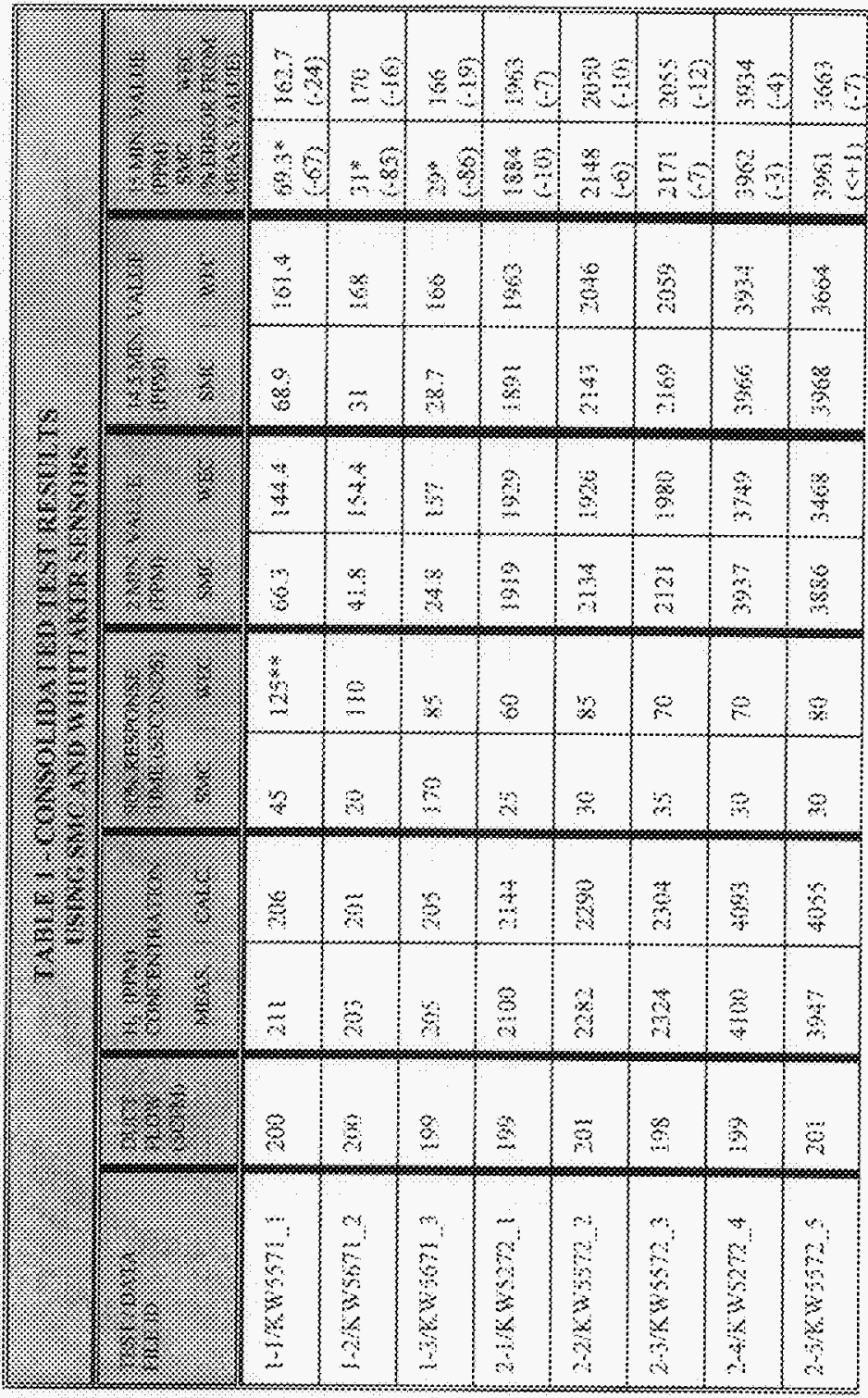




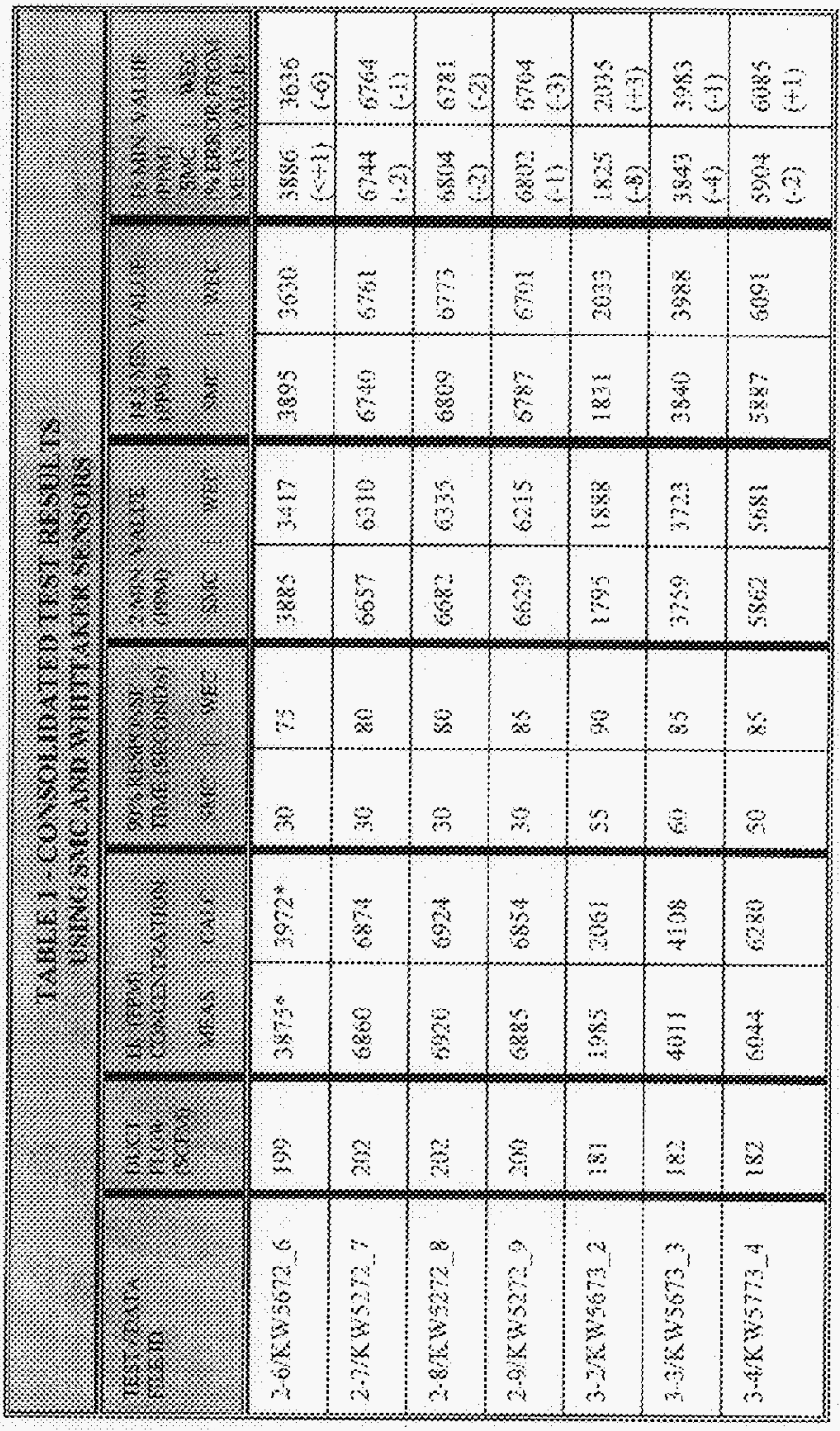

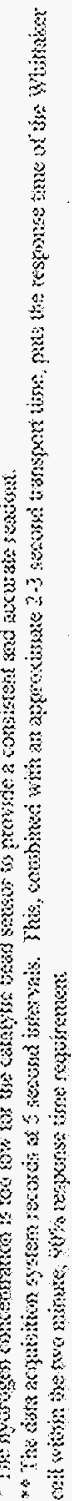




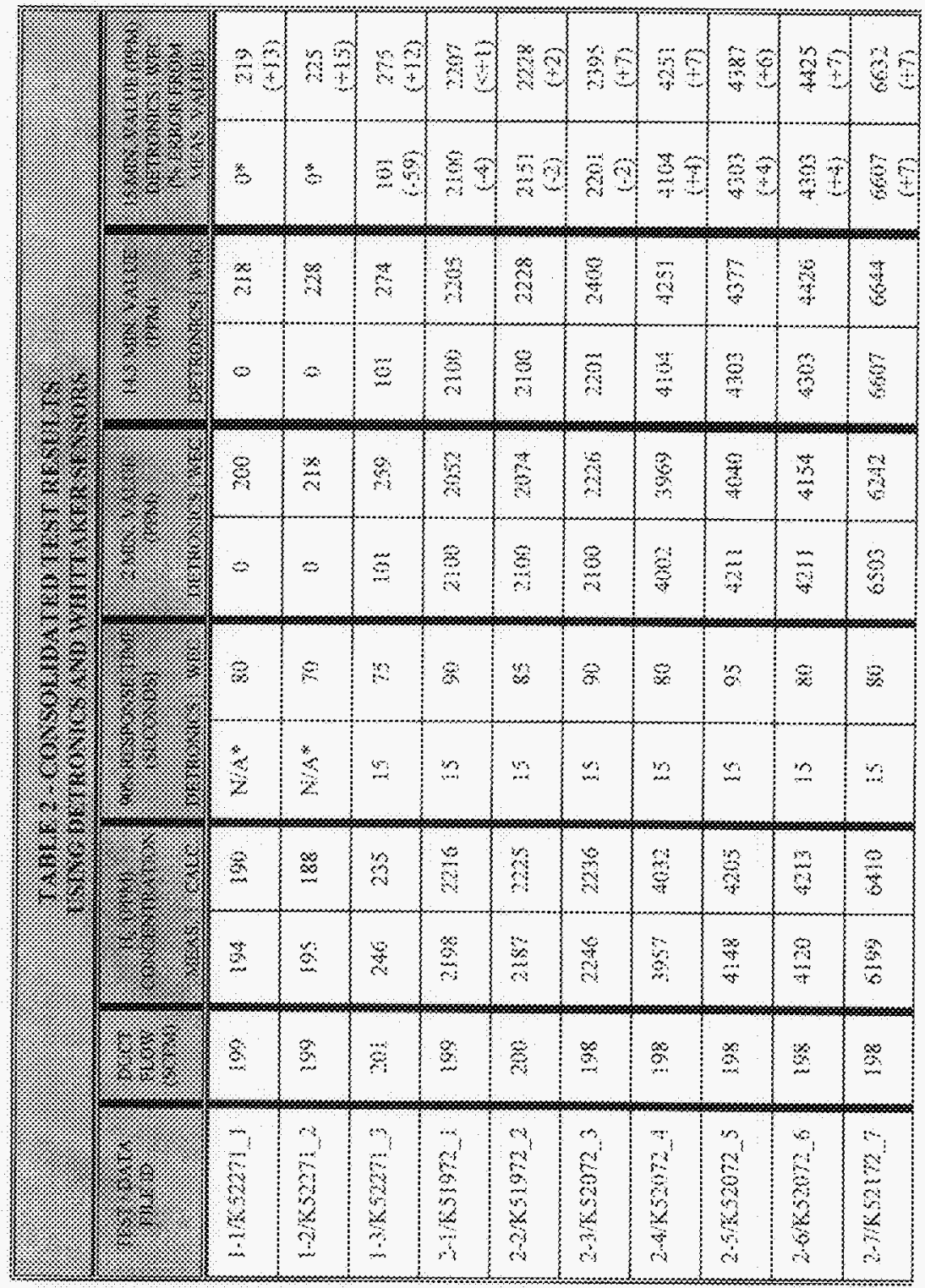



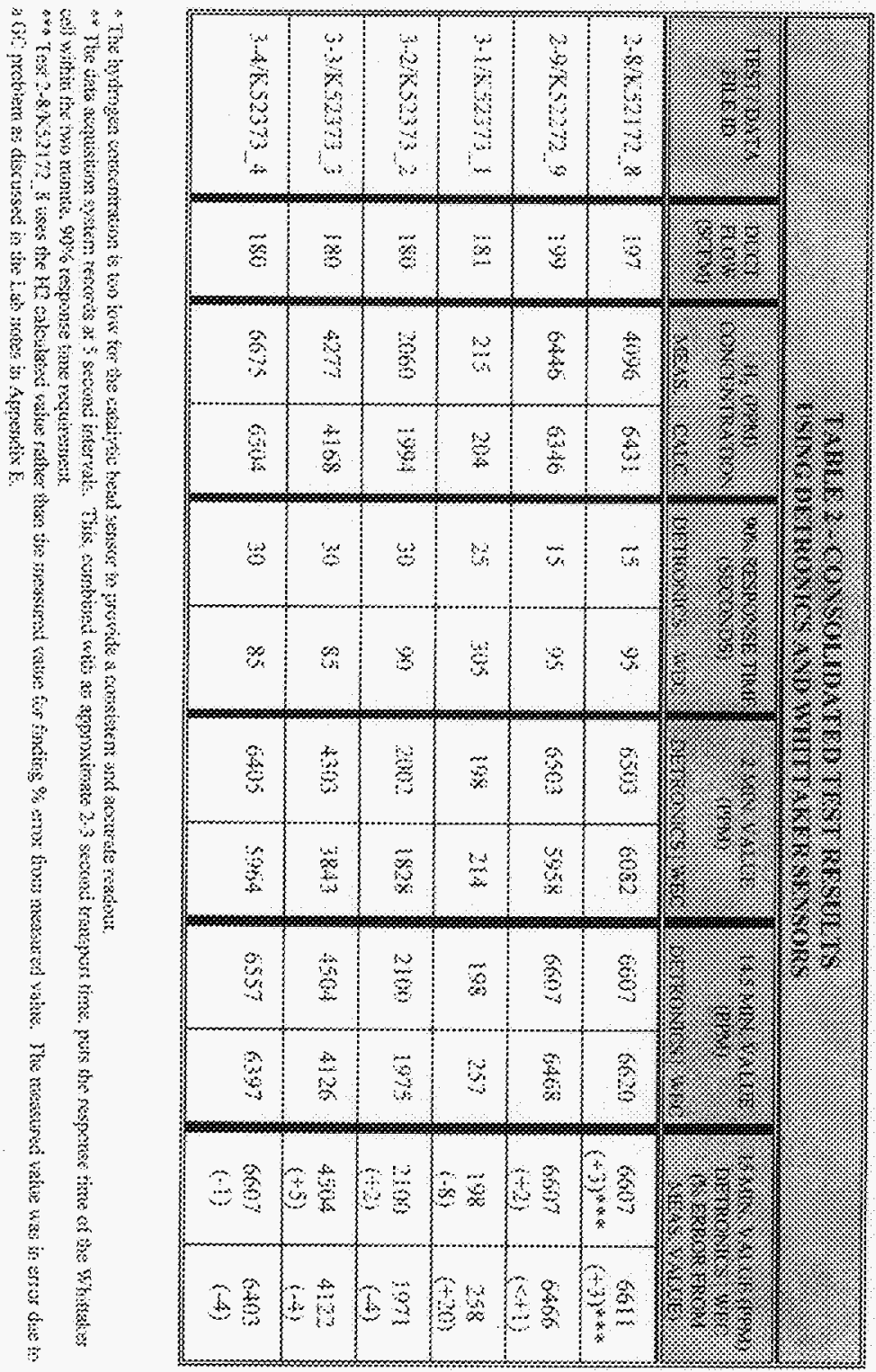


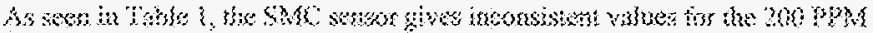

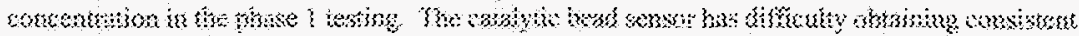

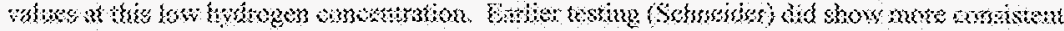

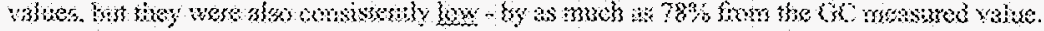

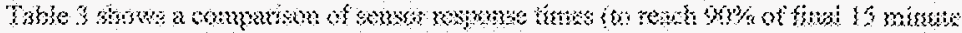

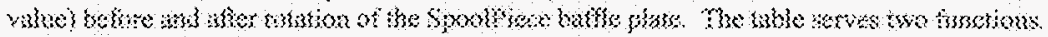

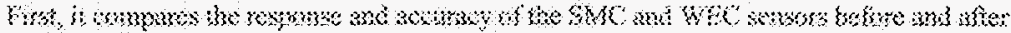

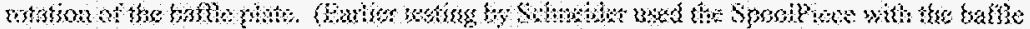

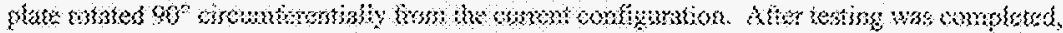

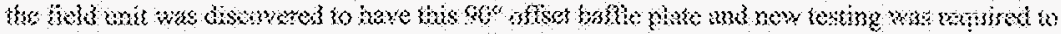

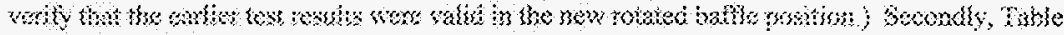

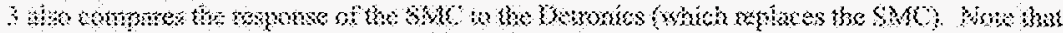

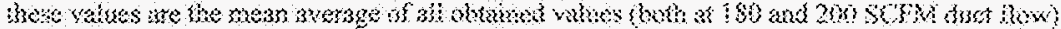

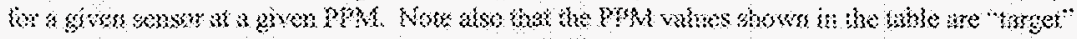

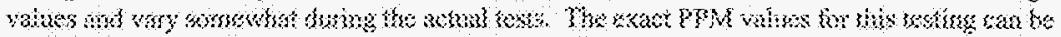

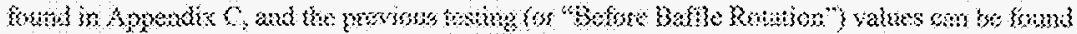

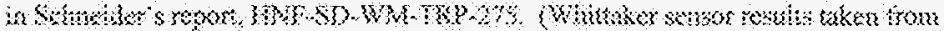

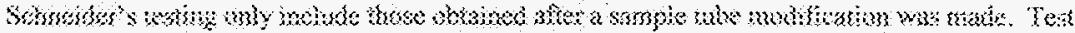

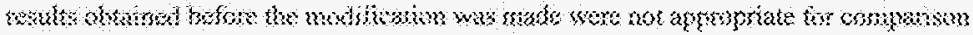
foposise;

\begin{tabular}{|c|c|c|c|c|c|}
\hline & \% & 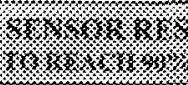 & $\frac{1}{x-m}$ & 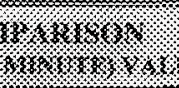 & \\
\hline \multirow{2}{*}{ 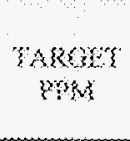 } & \multicolumn{2}{|c|}{ 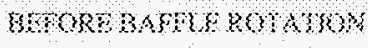 } & \multicolumn{3}{|c|}{ 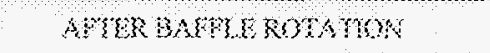 } \\
\hline & $\frac{\text { Sto }}{\text { (xesomsis) }}$ & 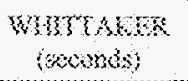 & $\begin{array}{c}S k x \\
\text { gecosis? }\end{array}$ & 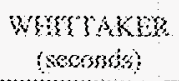 & $\begin{array}{c}\text { Ptraghe } \\
\text { (sommin) }\end{array}$ \\
\hline 230 & 398 & $8 \times 3$ & 783 & 908 & 20 \\
\hline 2030 & 383 & 553 & $36=$ & 825 & IS.S \\
\hline 3000 & 285 & $3 \% 4$ & $3 \%$ & 312 & ISSS \\
\hline 6000 & 30 & 53.3 & 35 & 85,6 & $18 s$ \\
\hline
\end{tabular}

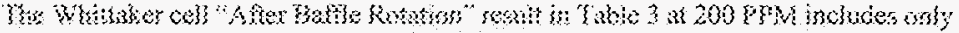

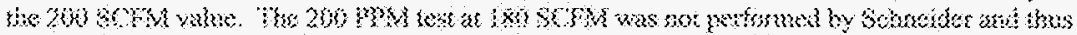

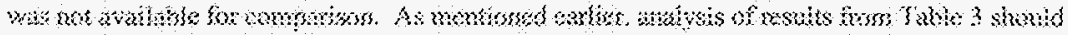

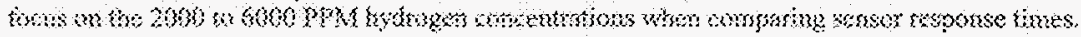




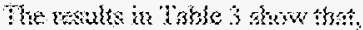

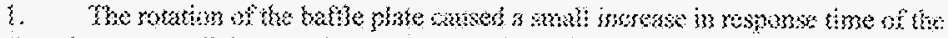

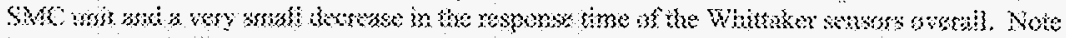

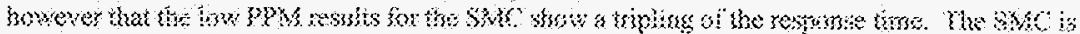

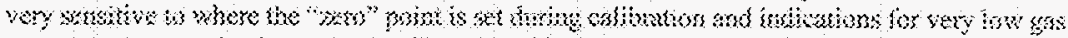

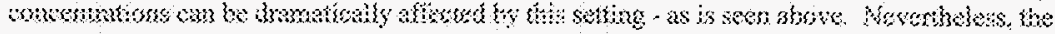

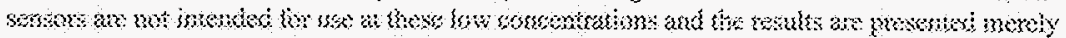

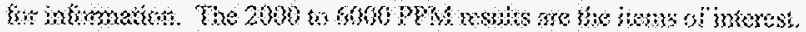

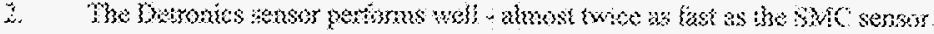

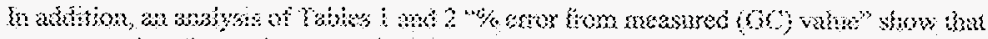

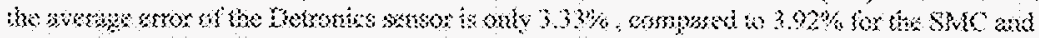

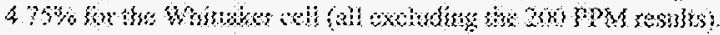

\section{THS XXophos}

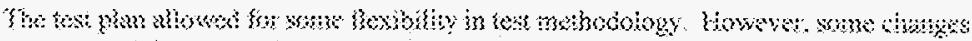

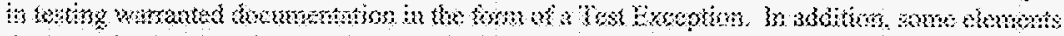

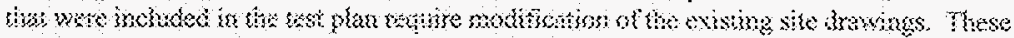

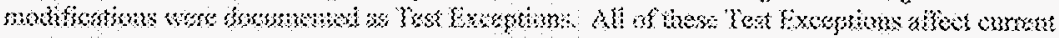

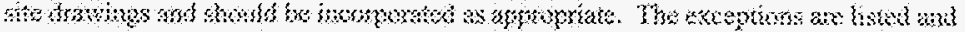

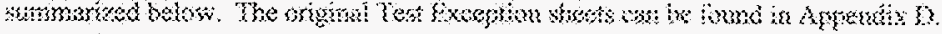

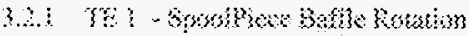

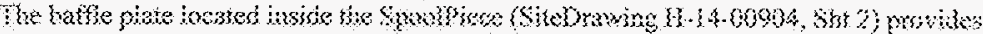

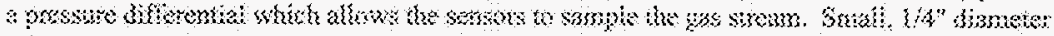

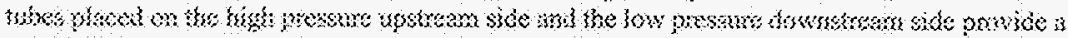

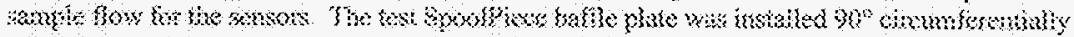

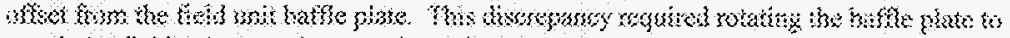

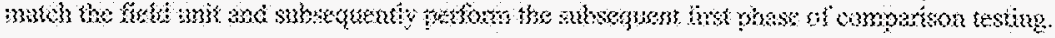

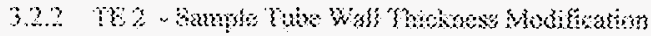

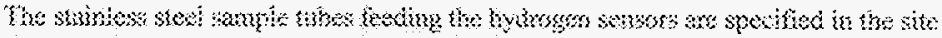

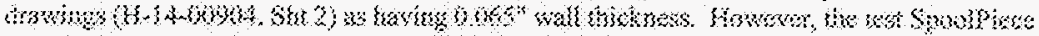

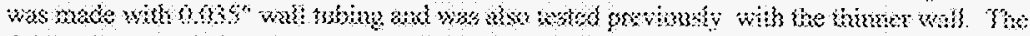

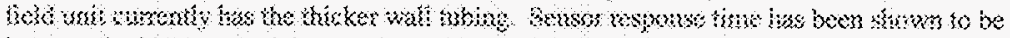

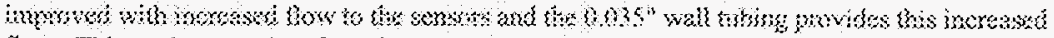

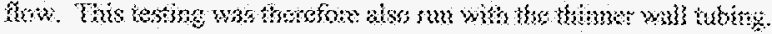

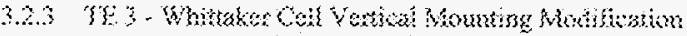

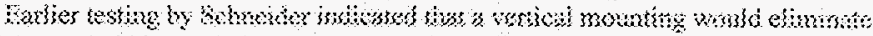

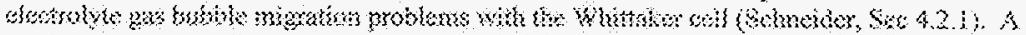

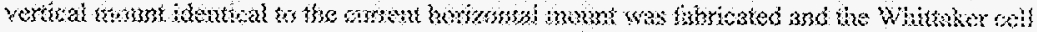




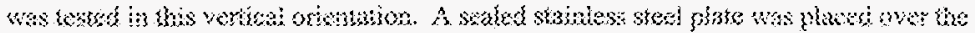
borkmon! mout.

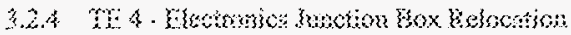

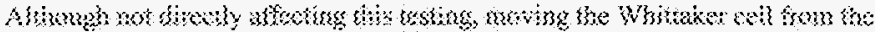

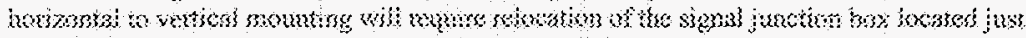

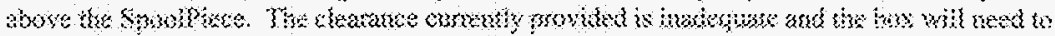

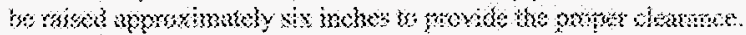

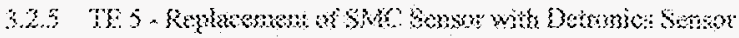

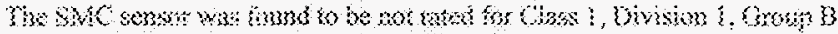

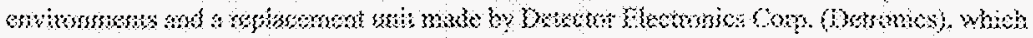

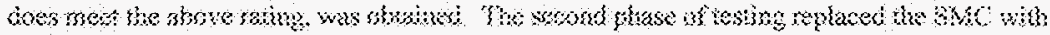
the Qestomice urit.

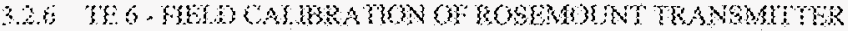

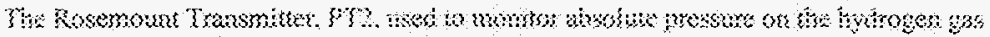

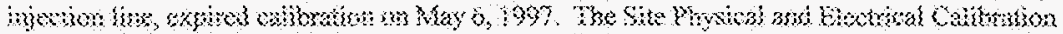

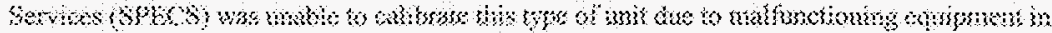

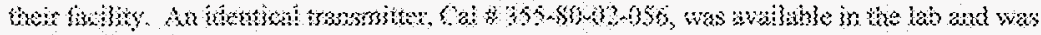

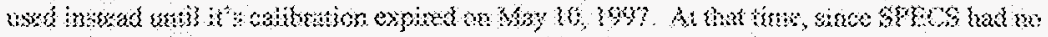

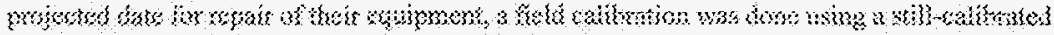

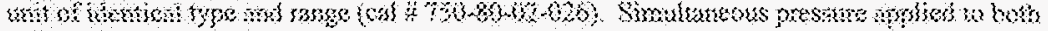

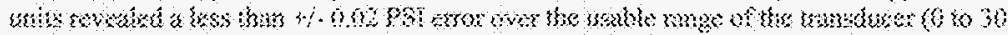

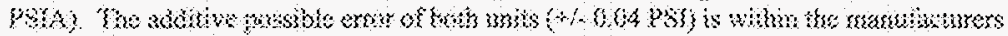

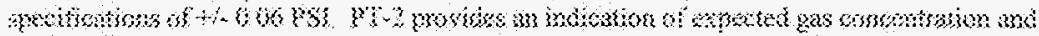

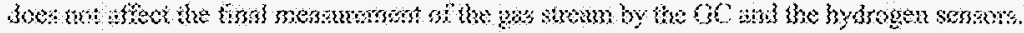

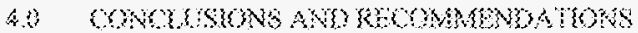

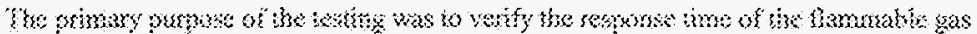

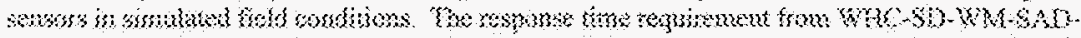

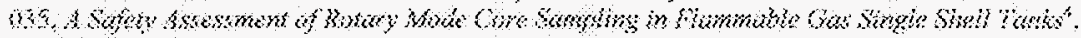

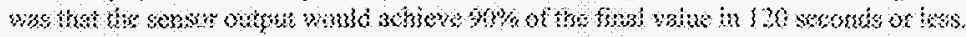

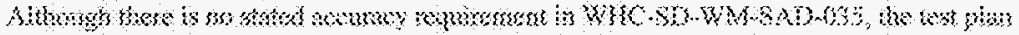

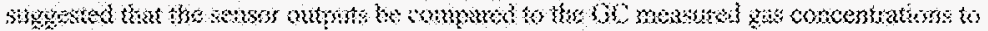

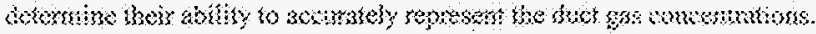




\section{8 कKOY}

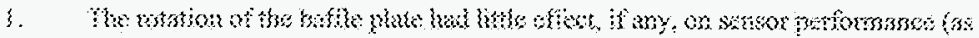

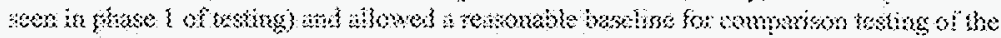
whors be phase 2

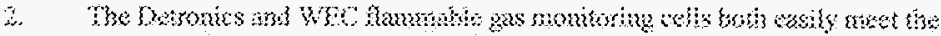

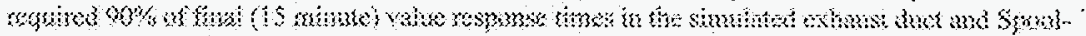
piese tho wh pressue ostitions.

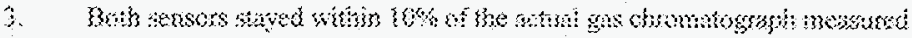

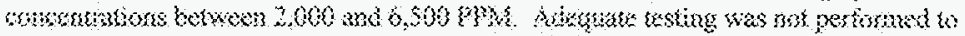

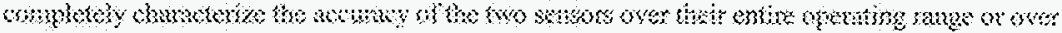

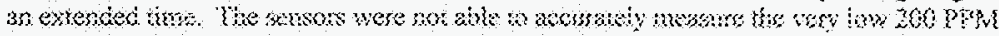

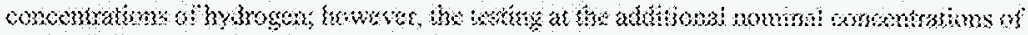

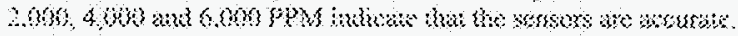

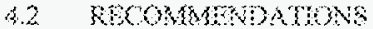

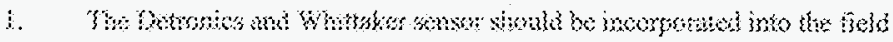
Spowbees asmby an med

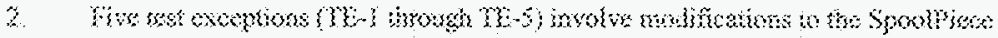

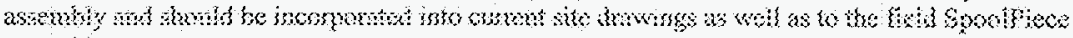
asonuto

\section{C\& BEFमEAOS}

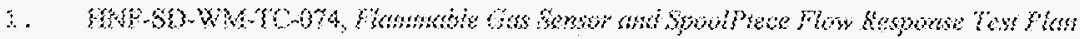

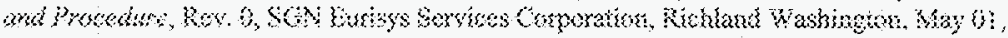
$19 \% \%$

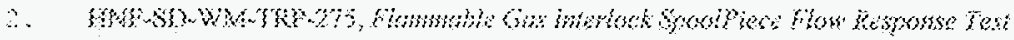

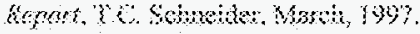

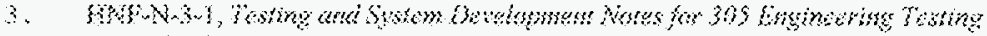

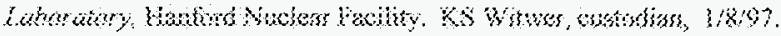

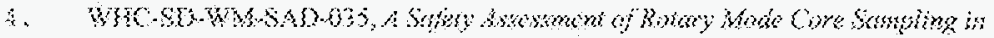

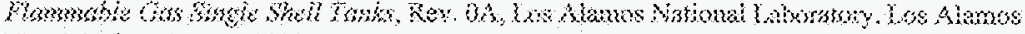

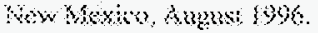



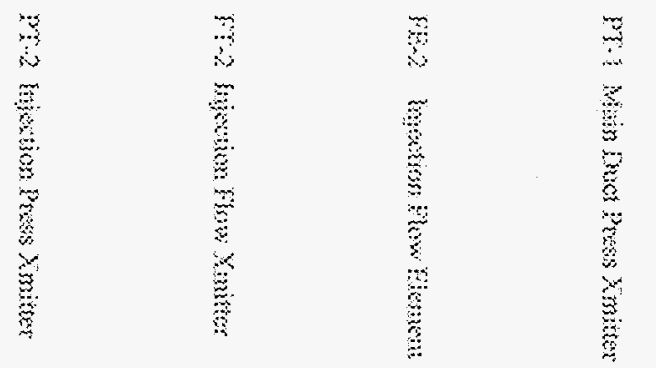

ב⿱艹

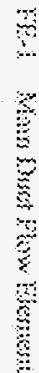

en 28\%

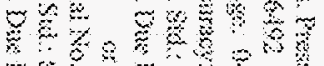

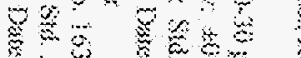
.... $\ddot{*} \mathrm{~s}$ .

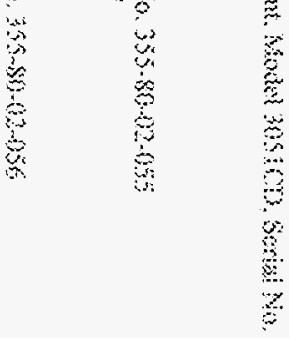

80 然离㛭 级 的 $4 \approx 0$

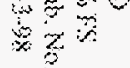

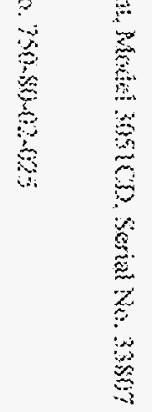

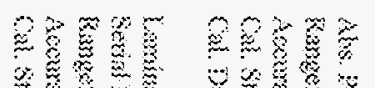

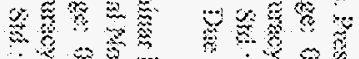

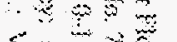

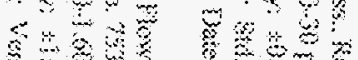
, w

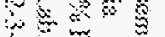

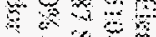
요의 xx $=0$

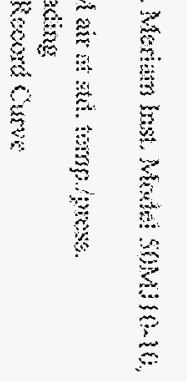

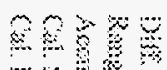

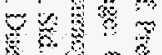
se

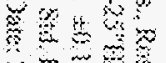

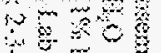
6 㠃

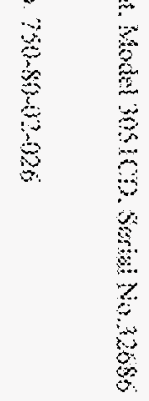

\% \& 둥 $6 \ldots$ … a. 3 的䇣 …… $\approx$

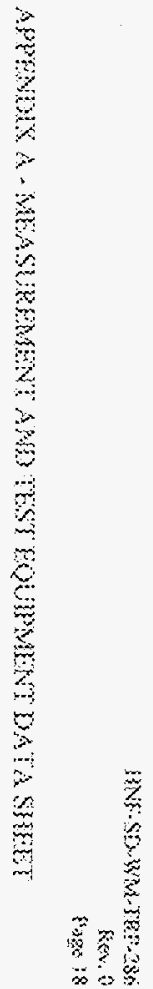




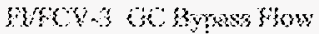

Fu or sample klow

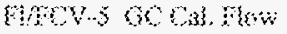

TS:-

$18 \%$

3 F. 3

Tr.

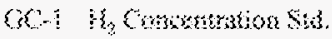

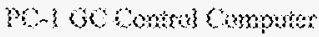

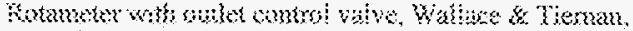
Swde: $32505391 \times 3$

Repoe $025 \mathrm{Ct}$ atr

Aommoy. zl\$o TS

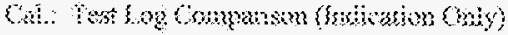

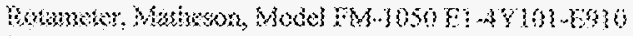

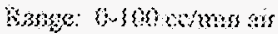

kcouacy: $5 \% \%$

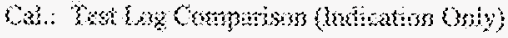

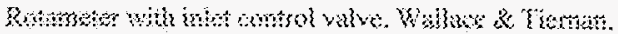

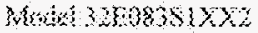

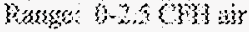

Lecuros: $\pm 10 \%$ \&\%

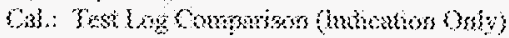

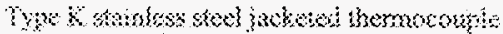

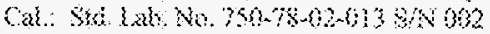

Cak Dwe Date: 2.598

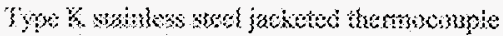

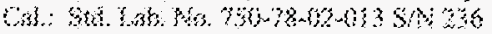

Gul Sue Dow 2058

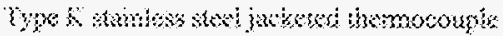

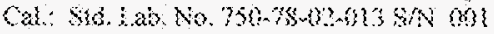

at The rmo: 25.98

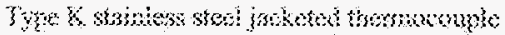

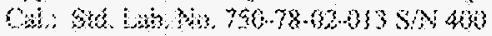

Cul Oos boss: $2-503$

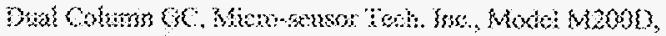
Seas $\times 5.150313$

3mot 10630 bon pros

Somms. $10 \%$ kentong

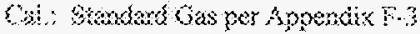

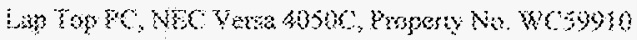
Range: $\mathrm{W} A$

Aommo. WA 
M- 1 Symo Shat hope:

WT- Whinker Sis Com:

NG-5 Whome smow

Ne2 $3 \mathrm{MC}$ Sonsor

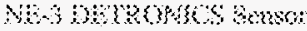

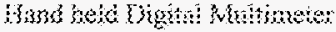

Reremate Rotamere:
A- $\beta^{\circ}$ )

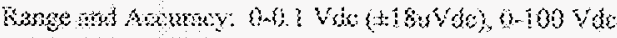
(103x)do) Th (c)

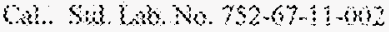

Gu. Dto buk: 2-54

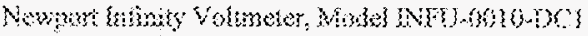

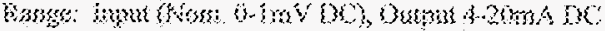

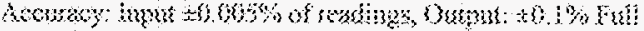
Siake

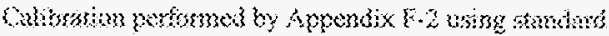

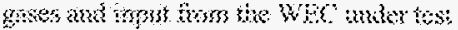

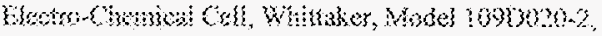

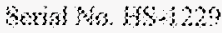

Risnge: $0.70 \% 3$ t. by Yolums

Acouray; to2\%, Abotito

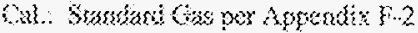

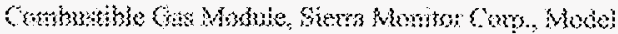

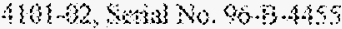

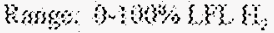

Accimsoy: $x$ ors

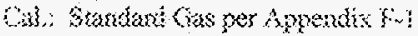

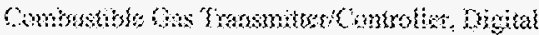

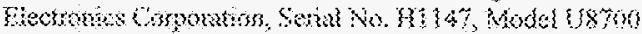
Range 9 -16m\% In Combustibe Sas

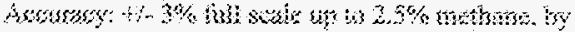

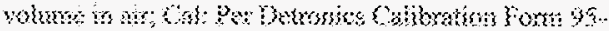
$3390-4 \%$

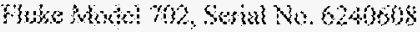

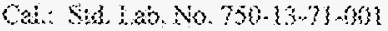

ut frge Mre: 1206.97

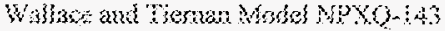

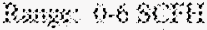

keowey $+10 \%$ pes

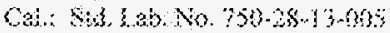

Cat. Ghe ante: $01-23-98$ 


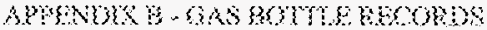

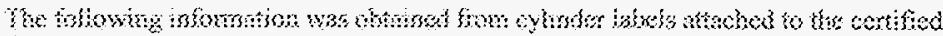

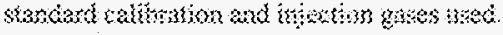

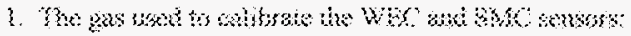

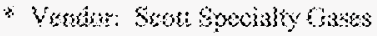

* inno. oss\%

* Perjes bis ks Y7mo

* Culinder leon No.

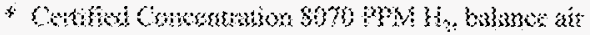

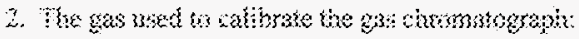

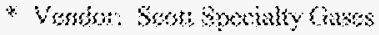

* Po No MOS XVVAl210\%

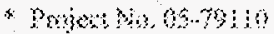

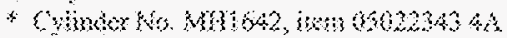

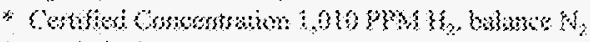

* Auatutwi Acumey to

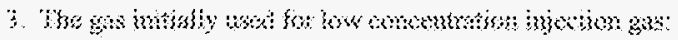

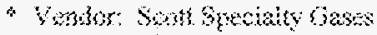

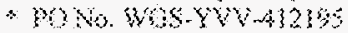

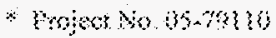

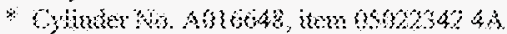

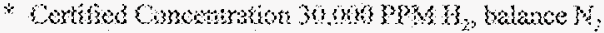

$*$ Andytion Asomasy $28 \%$

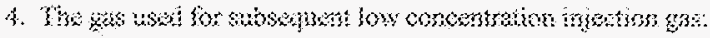

* Vender Sond boming hoso

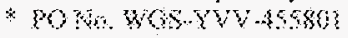

* Poject No or -37904

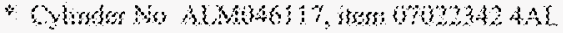

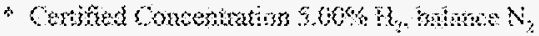

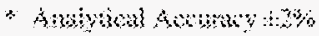




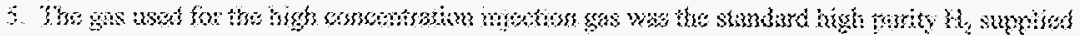

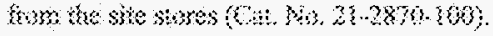

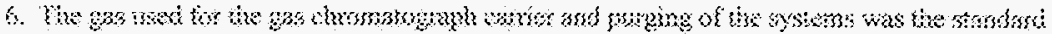

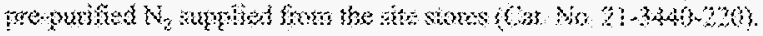




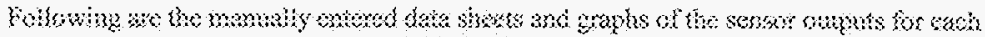

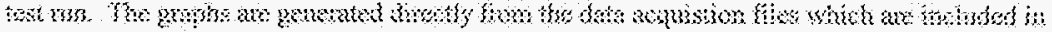
Anpotises. 


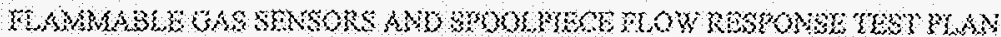
ANO sROCBubs:

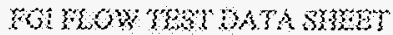

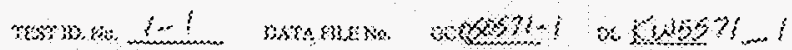

$\operatorname{sic} 3 \mathrm{sex}$

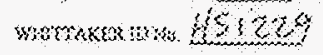

monocrono $A / A$

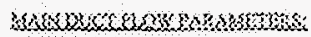

sowomonsw $20 \%$ sm

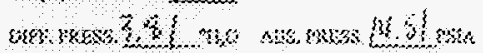

arsuseronow 200 ow

rese 34 is:

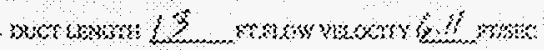

rexsmat rat $2,83 x$

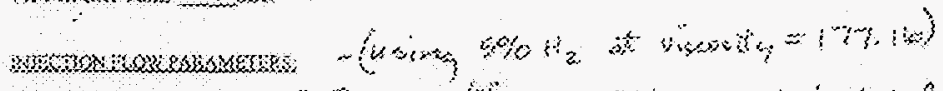

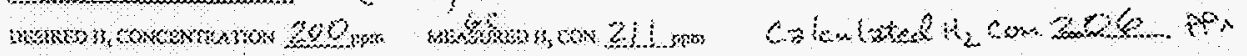
W\%

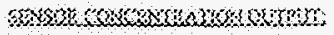

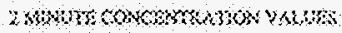

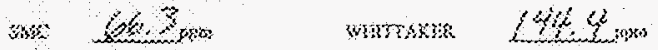

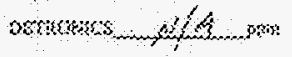

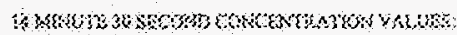

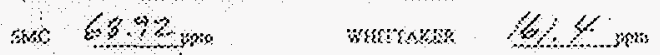

womstos $N / 2$ ion

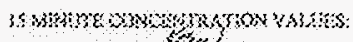

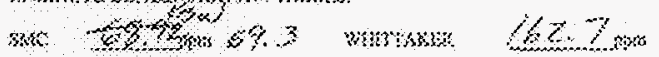

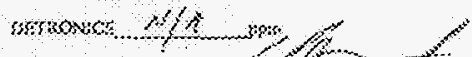

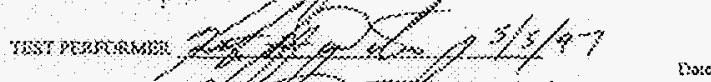

3ow wherks

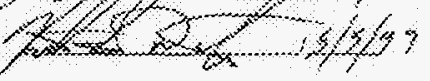

Exi: 
ma: $50-2 M-1+23-26$

isen 1

:oge 25

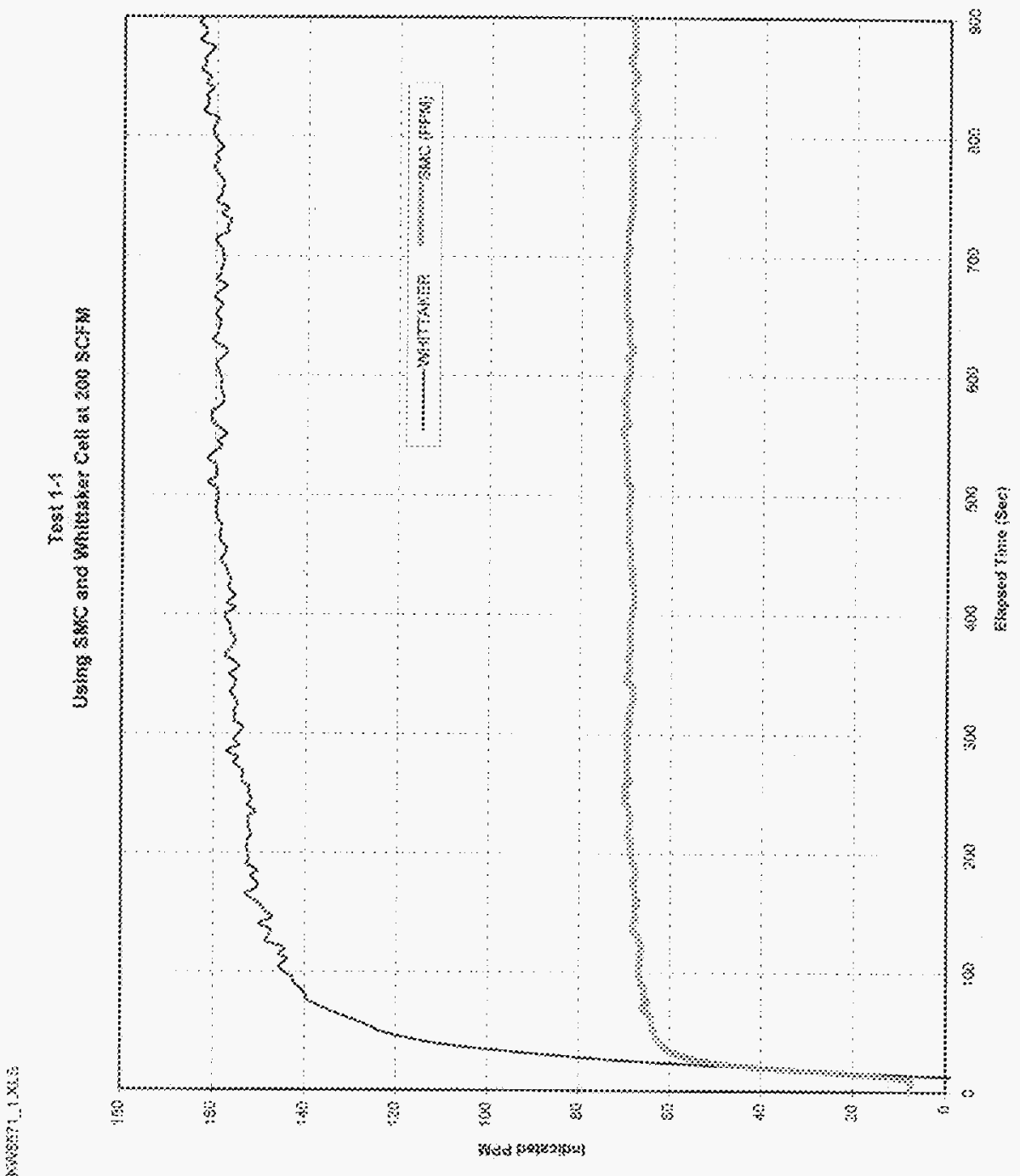




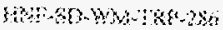

2.s. 59

$\operatorname{pog}: 26$

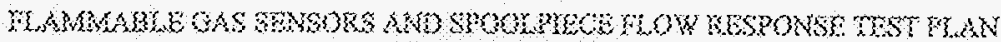
AND MuS SESTrex

\section{WU WOW TET GAXA SWES}

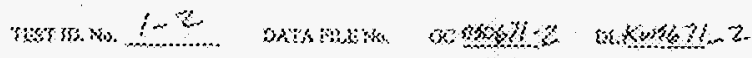

SBring. Bu, wet?

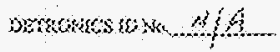

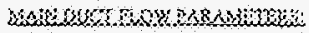

trowsos Row $200 \mathrm{cos}$

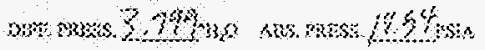

Gasunomow $700 \%$

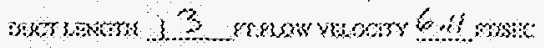

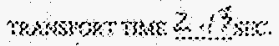

Tws: $78 \%$

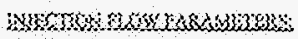

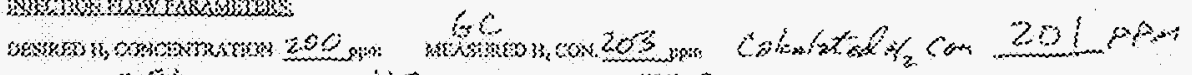

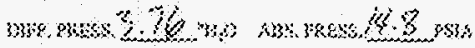
$3 \% 3.8 \%$

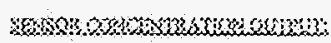

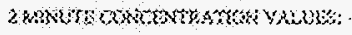

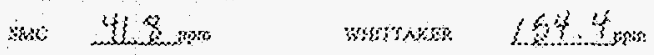

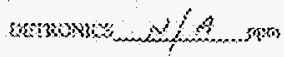

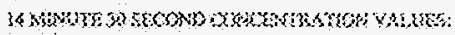

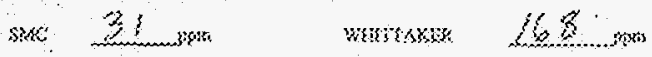

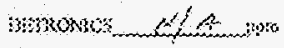

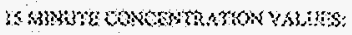

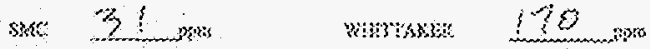

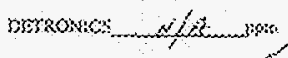

nscrosmon

rest mytenon

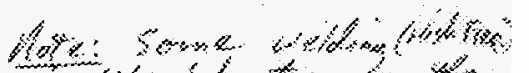

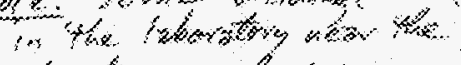

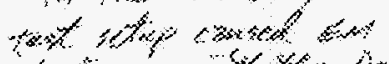
\%

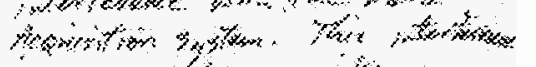

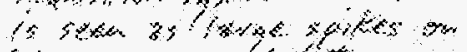

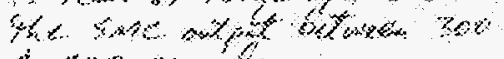
i. 5ow seariss.

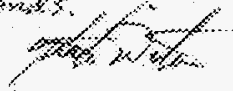




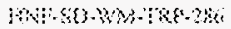

taxt 6

$\operatorname{lig} 2 \%$

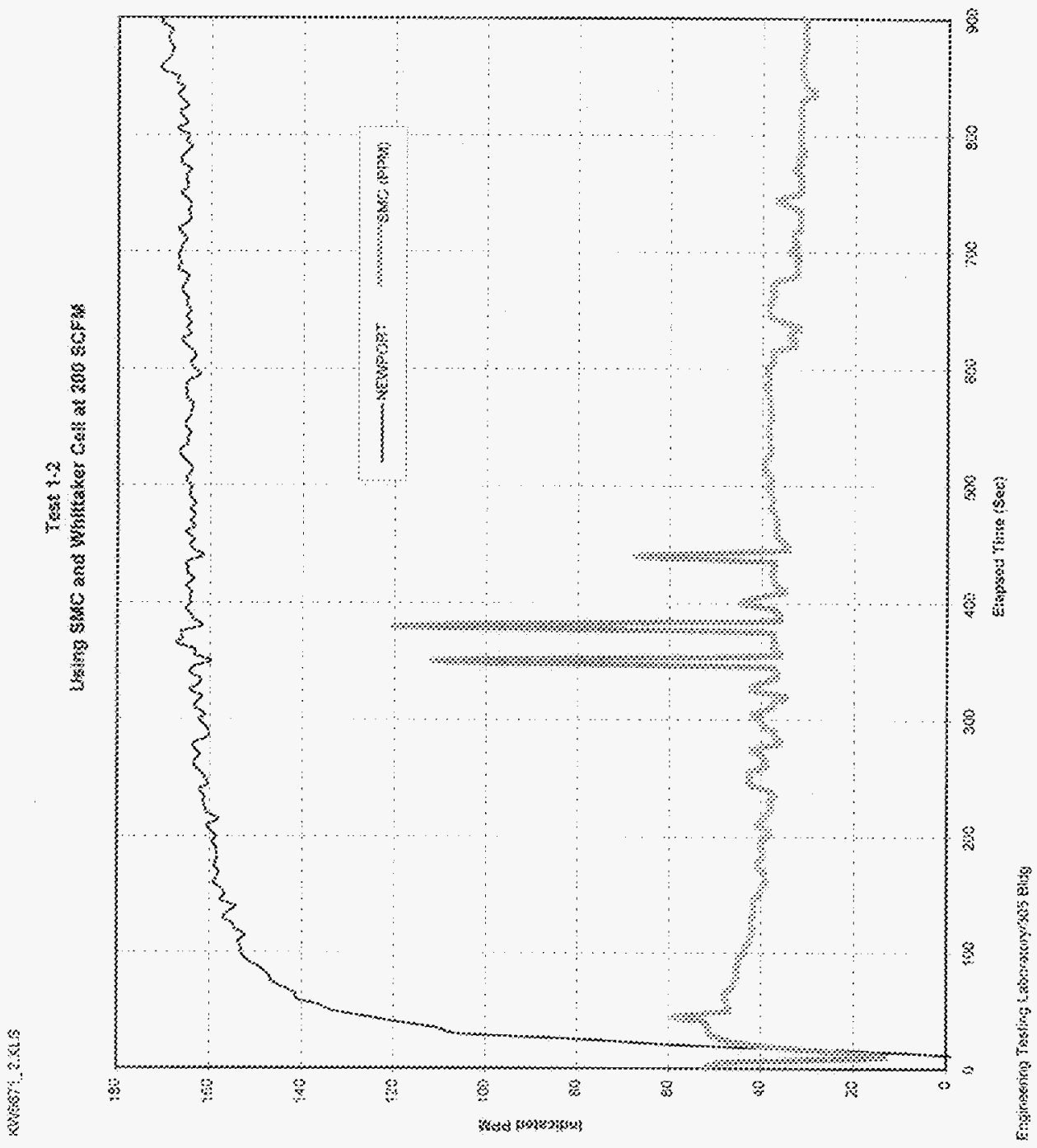




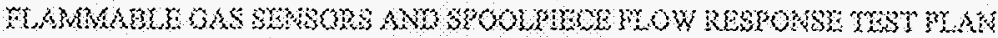
ANO B BOCSOURS

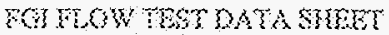

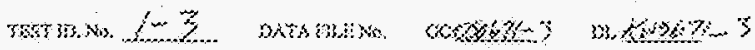

MSES Sh

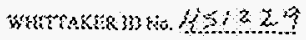

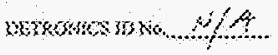

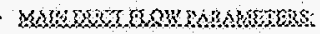

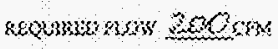

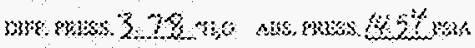

axtotokts meich viscors: xisus:

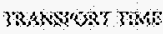
sec.

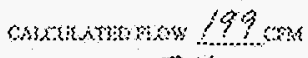

WW $74 x$

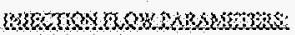

wasw

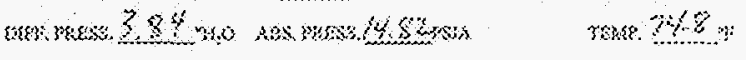

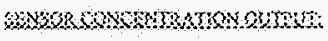

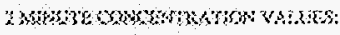

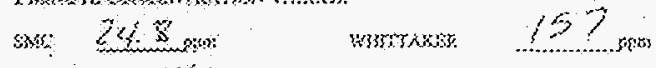

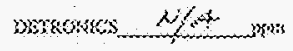

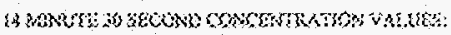

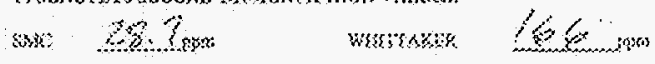

mowes $x /$ sos

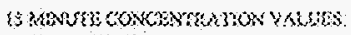

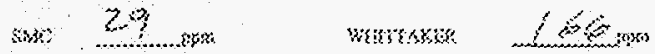

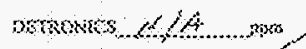

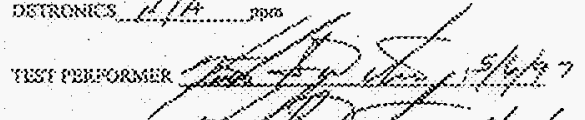

resromatrox

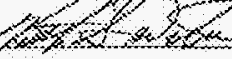


3-\{1\}-3) wh-

ReV :

yogen 28

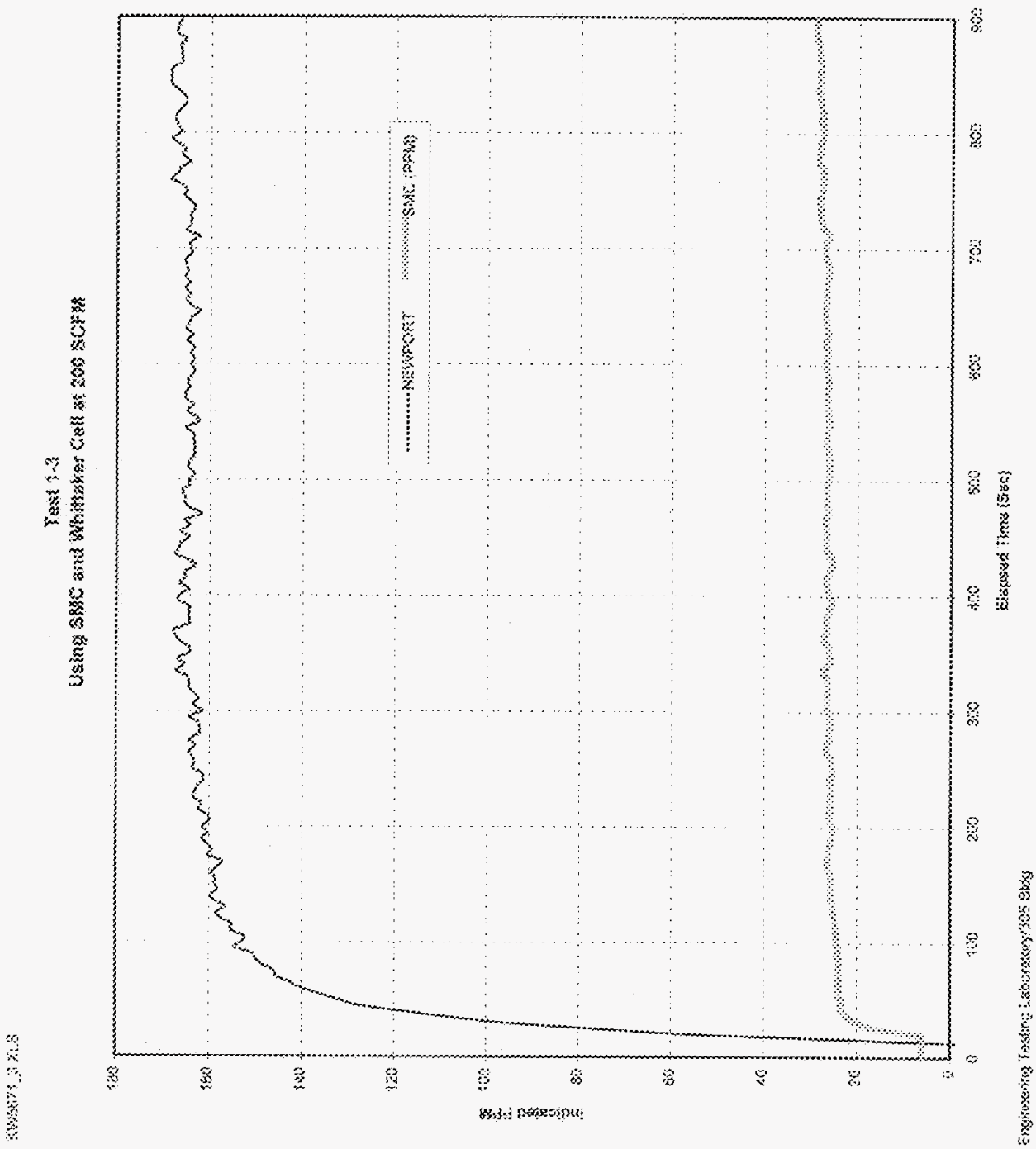




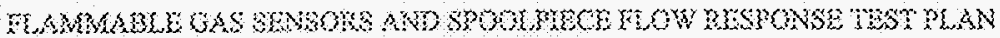

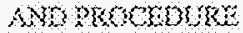

\section{MOHOW YOS DAOA SHEET}

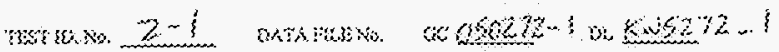

Whisho.

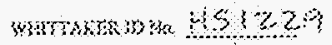

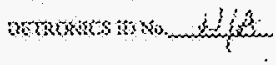

Martostrow

kewke tow $200 \%$

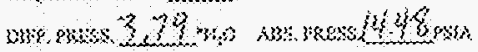

armaronow $39 \mathrm{~s}$.

rowe. $7 \mathrm{~s}$.

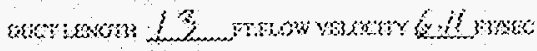

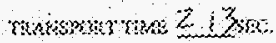

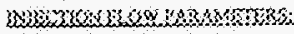

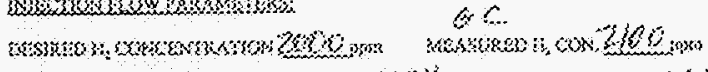

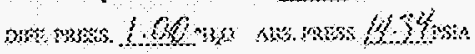

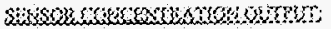

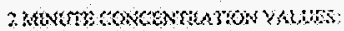

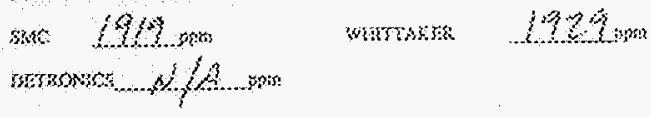

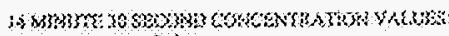

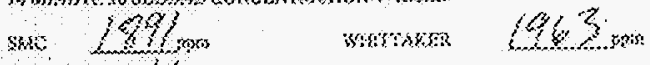

browses $\alpha / A$

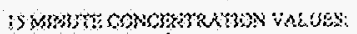

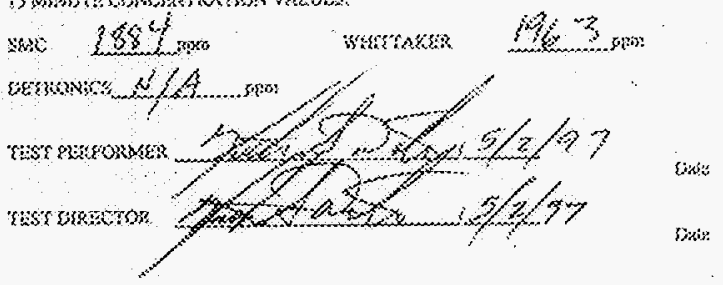


an-so-2M-Jep-286

asia

:soc: $: 3$

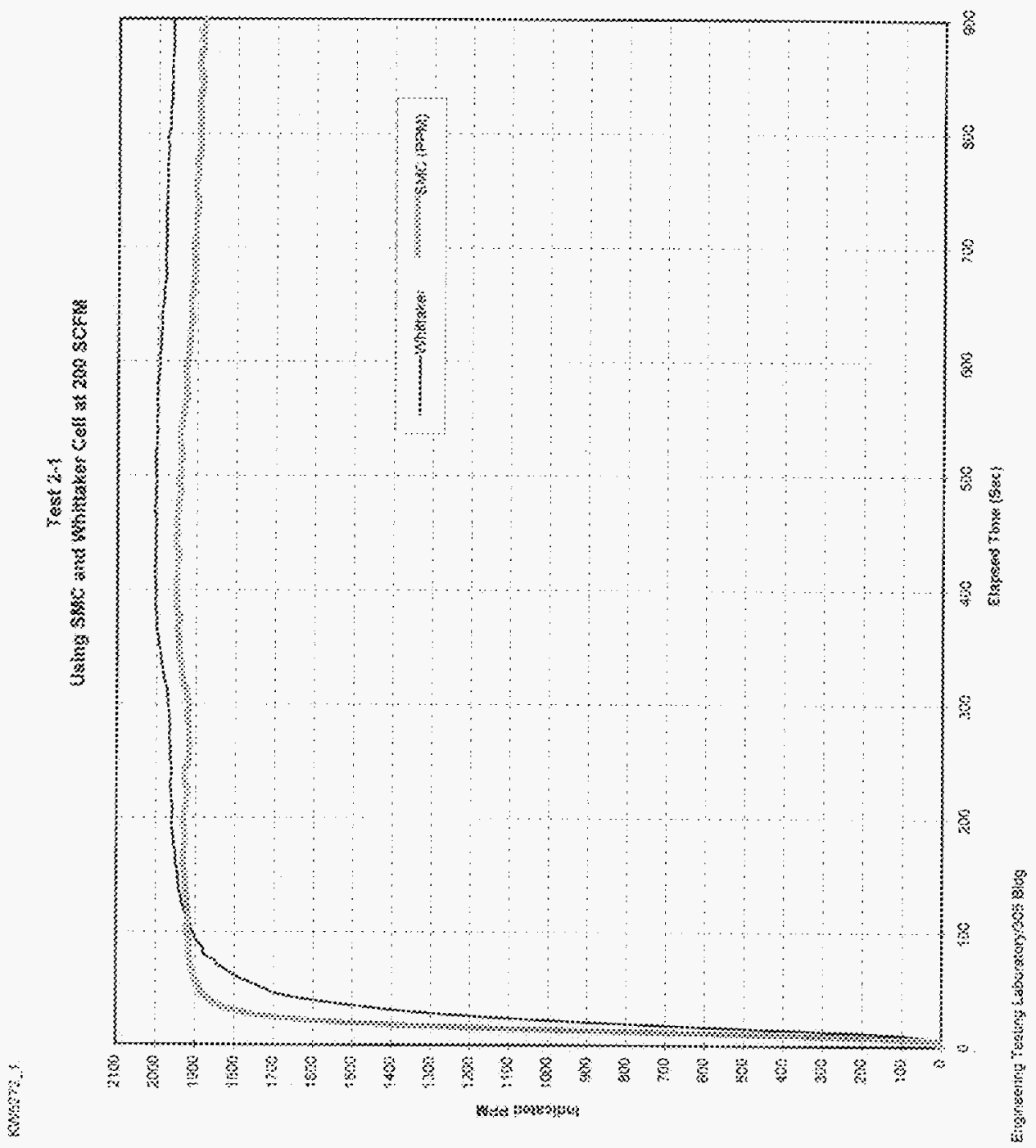




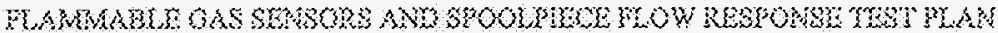
ARS POOOOSOUNO

\section{MU MOW TEST DATA SUEET}

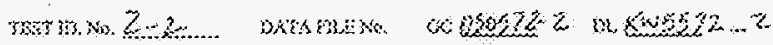

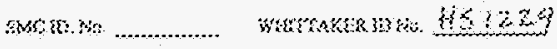

mowhow wo

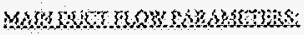

k6umisow 201 sm

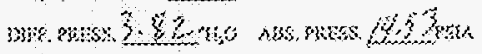

\section{acturanos 201 man}

rmisting

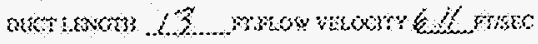

maxser thes $2: 3$ Hec.

HoTn

wow

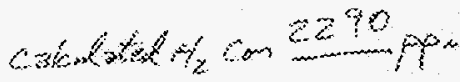

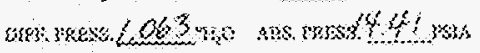
meke $2 y .4 \%$

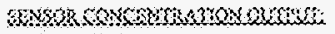

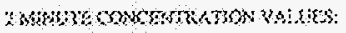

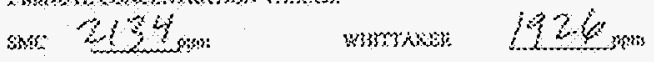

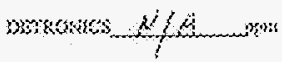

4h rasere

sw: z⿺13 man

mathes Non

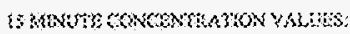

se 2118 mon 2950

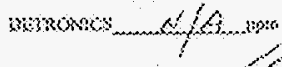

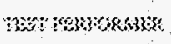

Tesrowberot
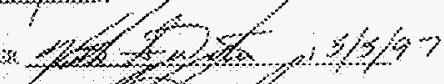


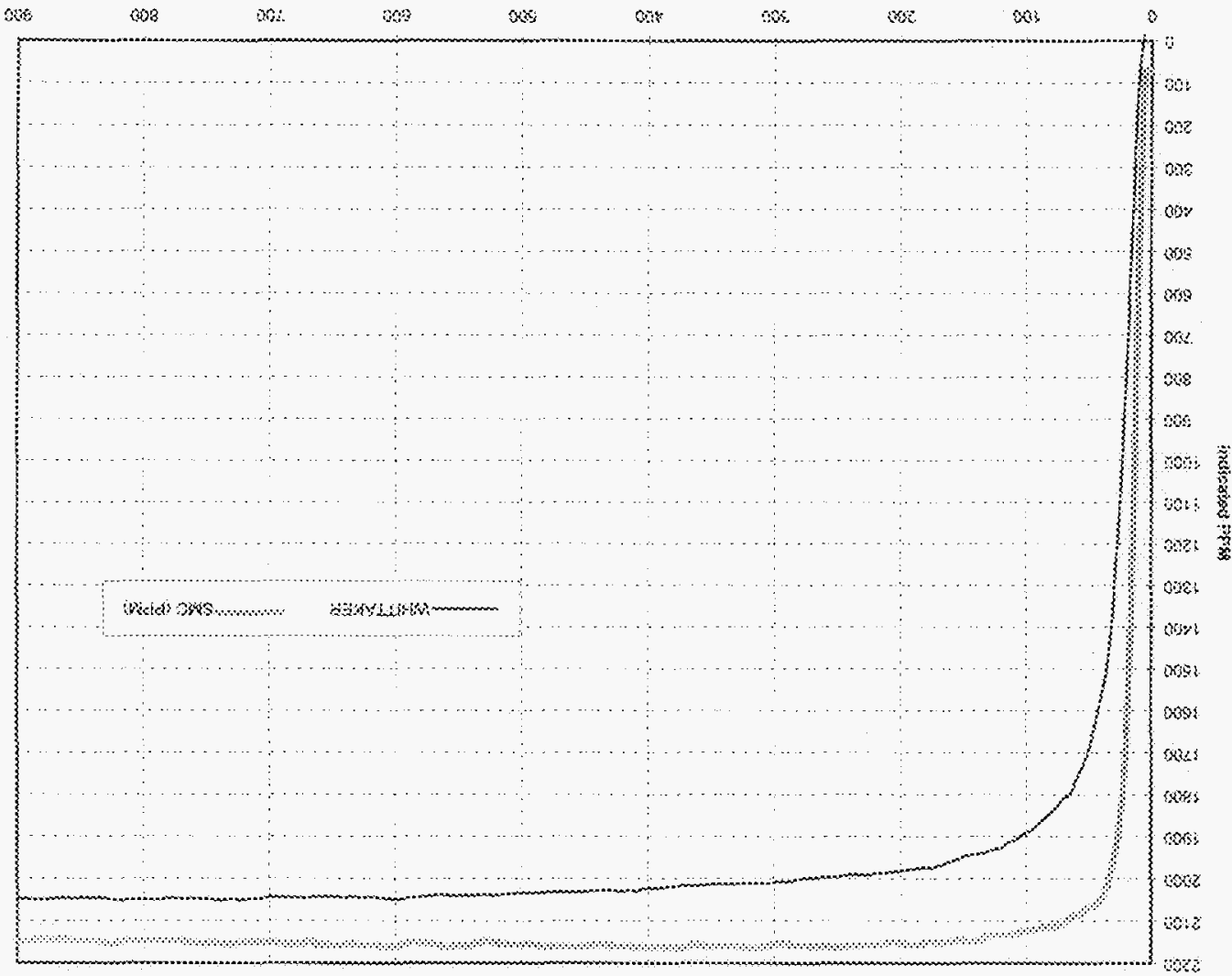

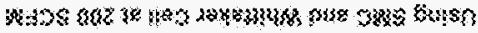

sones 


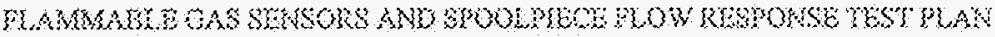
ANOSBOSOSUNS

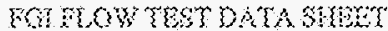

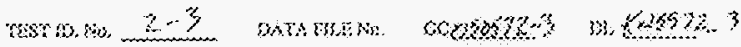

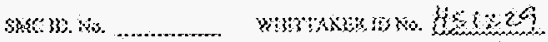

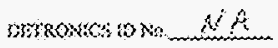

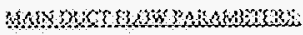

3\%)

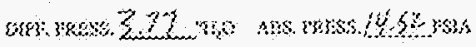

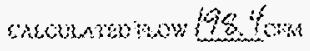

mas $73,2,2$

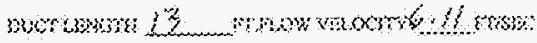

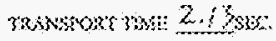

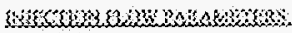

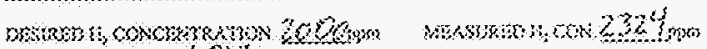

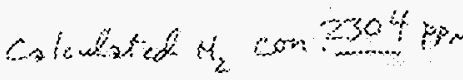

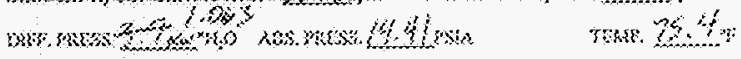

Nowosowotroxumswom

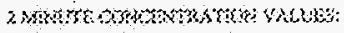

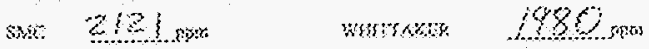

botweres givis

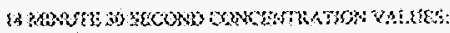

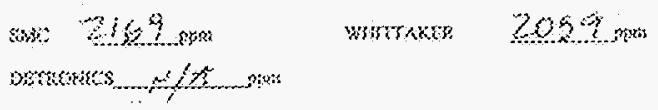

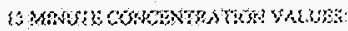

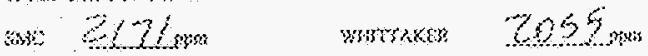

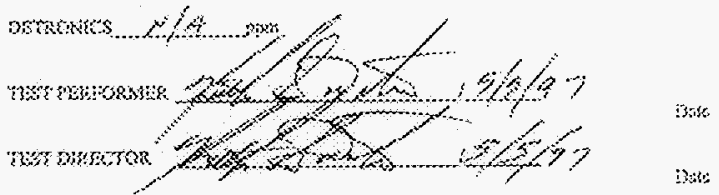




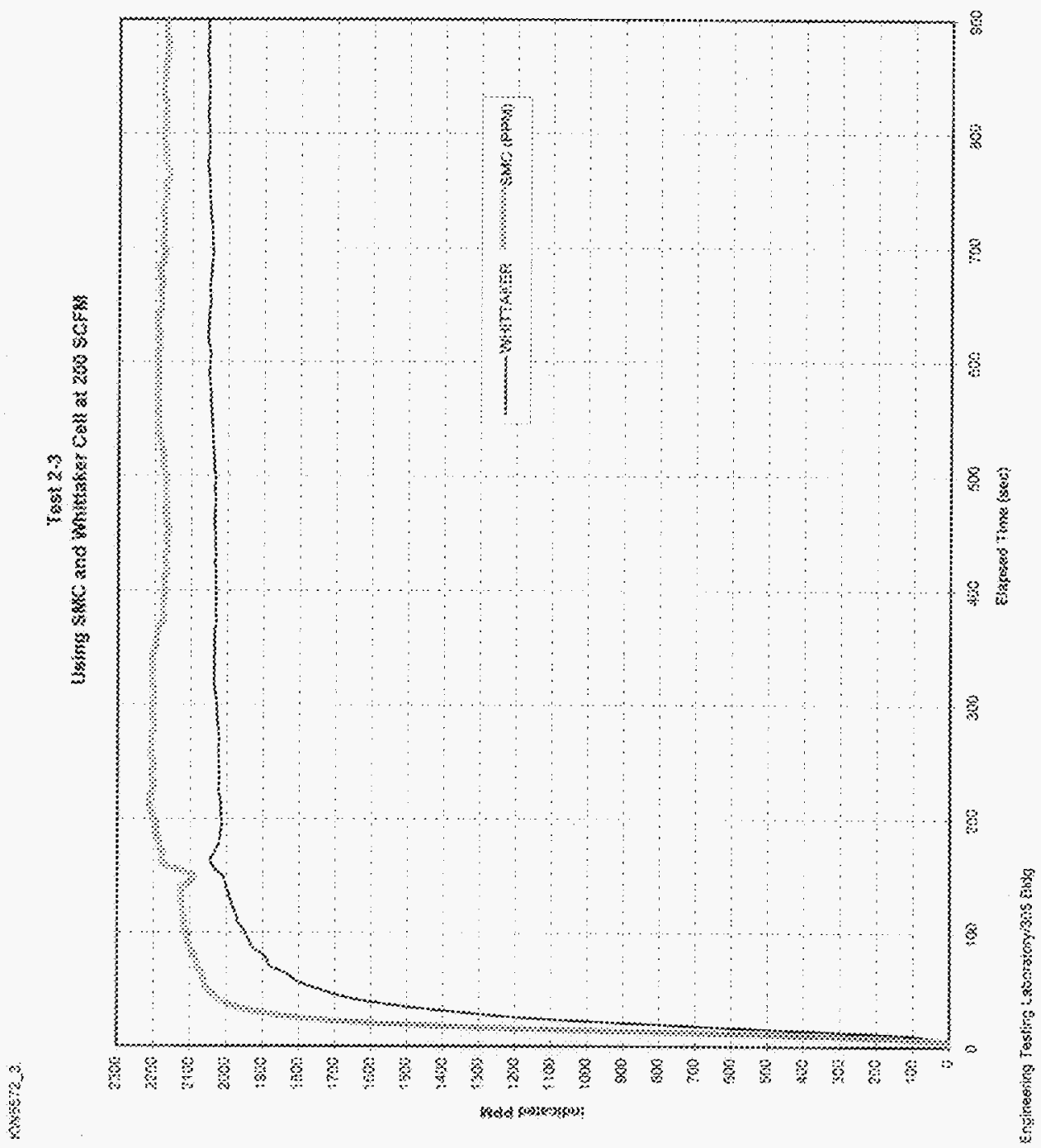




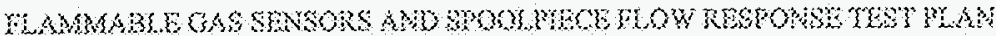

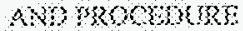

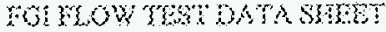

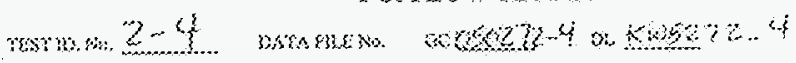

swe $3 \mathrm{n}$ ike

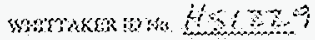

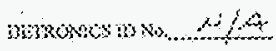

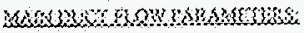

Ropmuterow $3260 \mathrm{~m}$ acsinsos

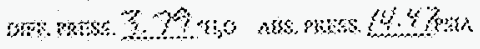
rasing 797

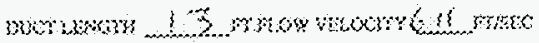

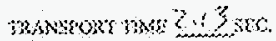

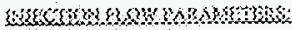

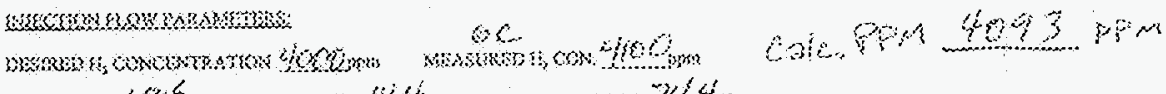

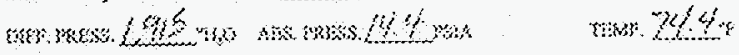

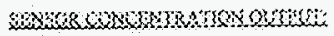

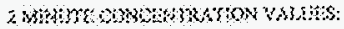

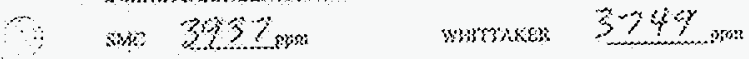

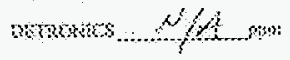

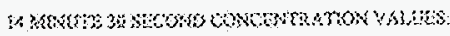

swe 3966 worno 2039

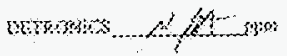

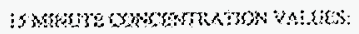

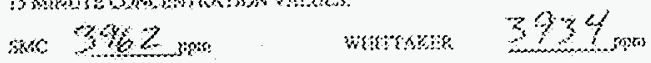

nowow Af

wromenm

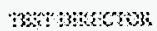

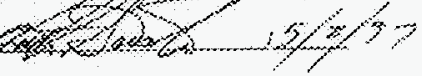




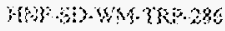

Brav.

3 itige 37

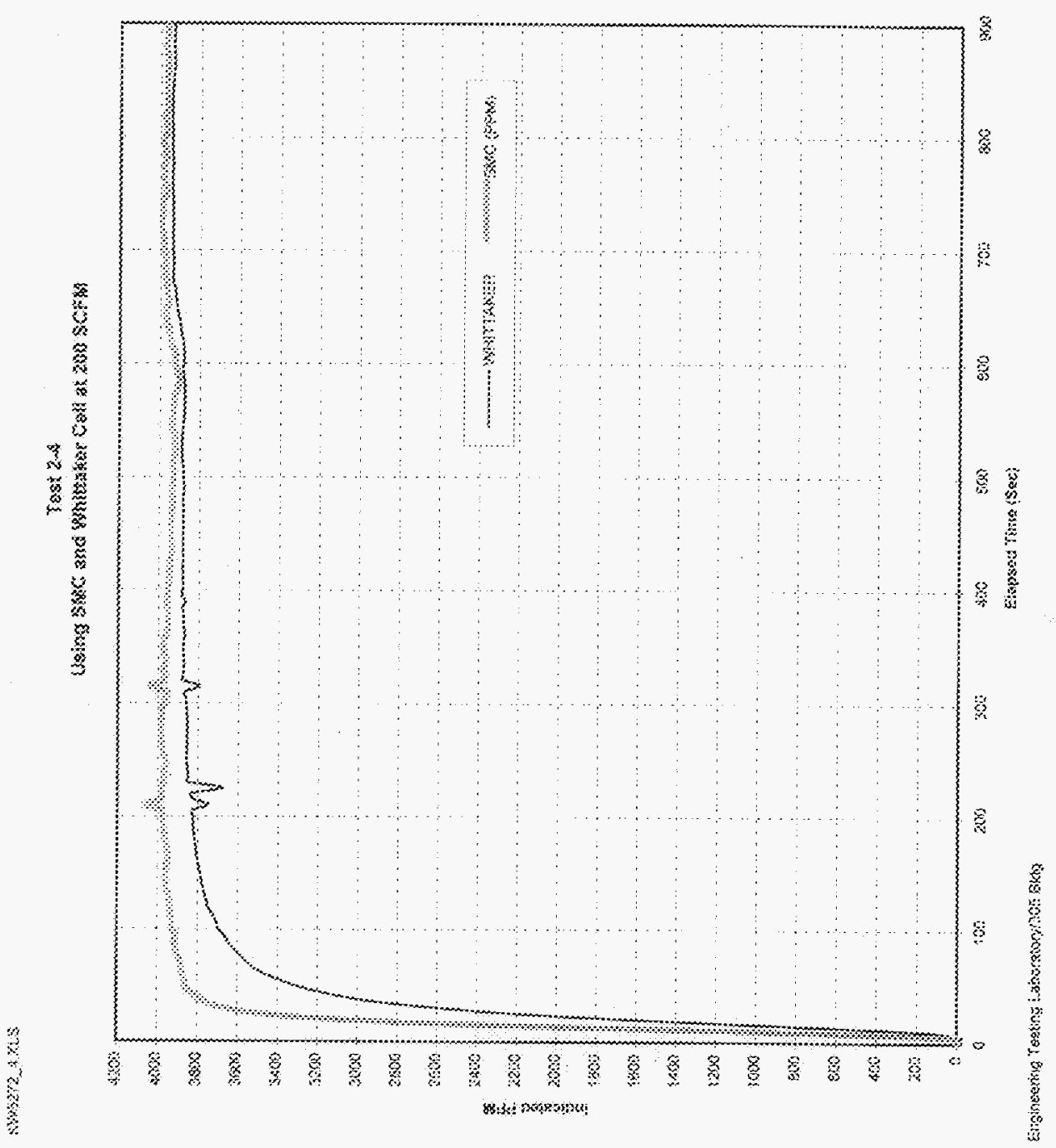




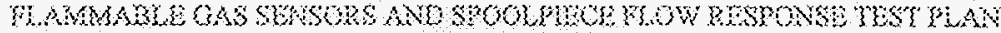
AMO $\mathrm{POOMYHE}$

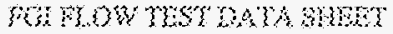

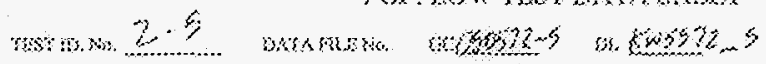

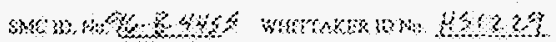

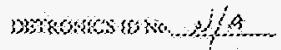

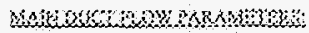

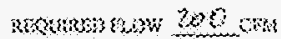

mansomow $20 !$

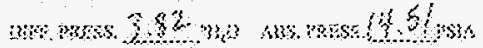

rist? $7 ?$ ?

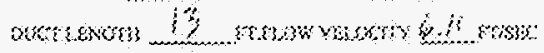

mansmorom 2 tasse.

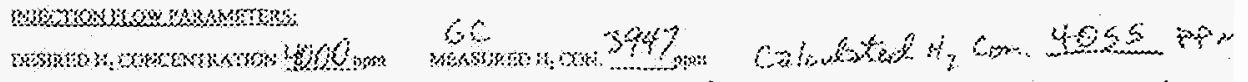

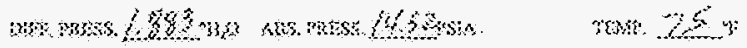

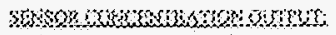

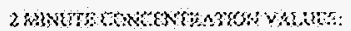
$360 \quad 3986$

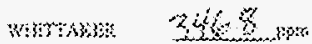
arouns

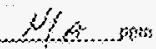

ix

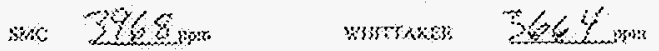

mownes

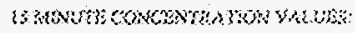

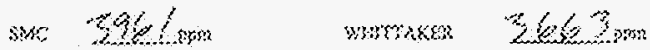

warkess N/A

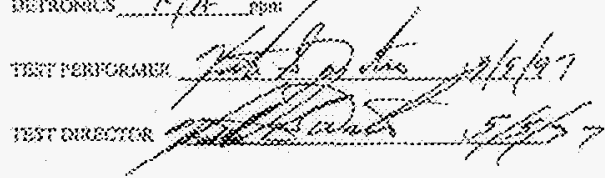




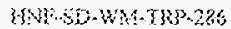

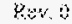

Pow 35

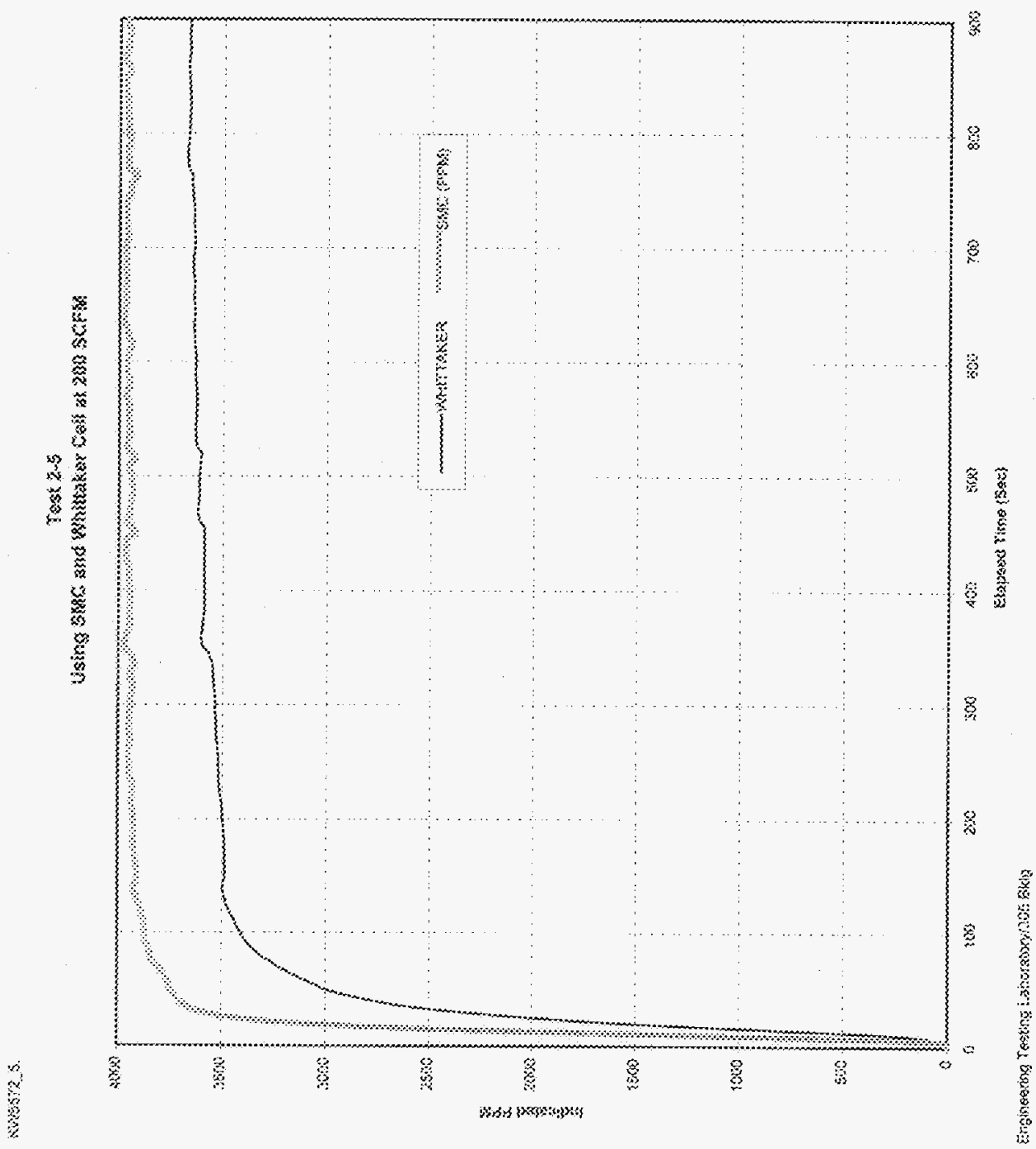




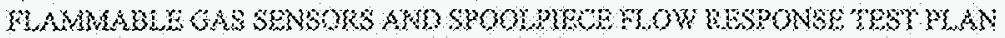
$\triangle N O$ WOOOHE

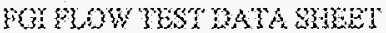

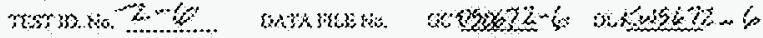

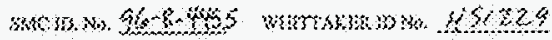

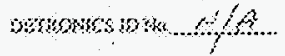

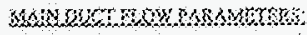

uscomos now $32 \mathrm{~s}$ on

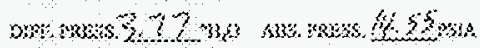

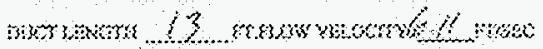

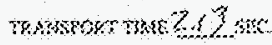

Wortomoswoskumo:

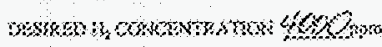

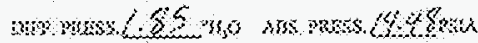

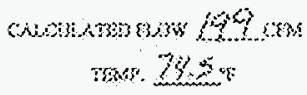

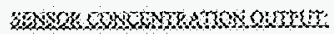

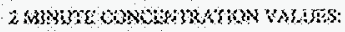

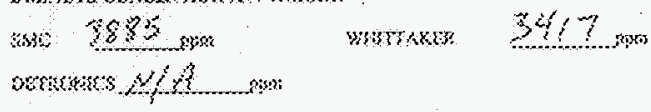

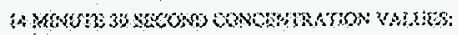

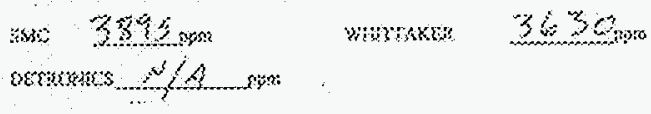

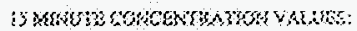

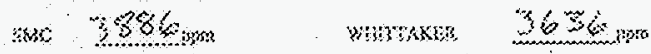

NBrosks $\times / A$

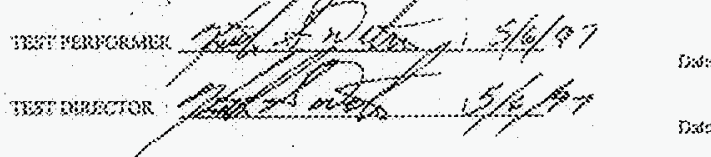




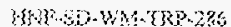

ger.9

Yose 4

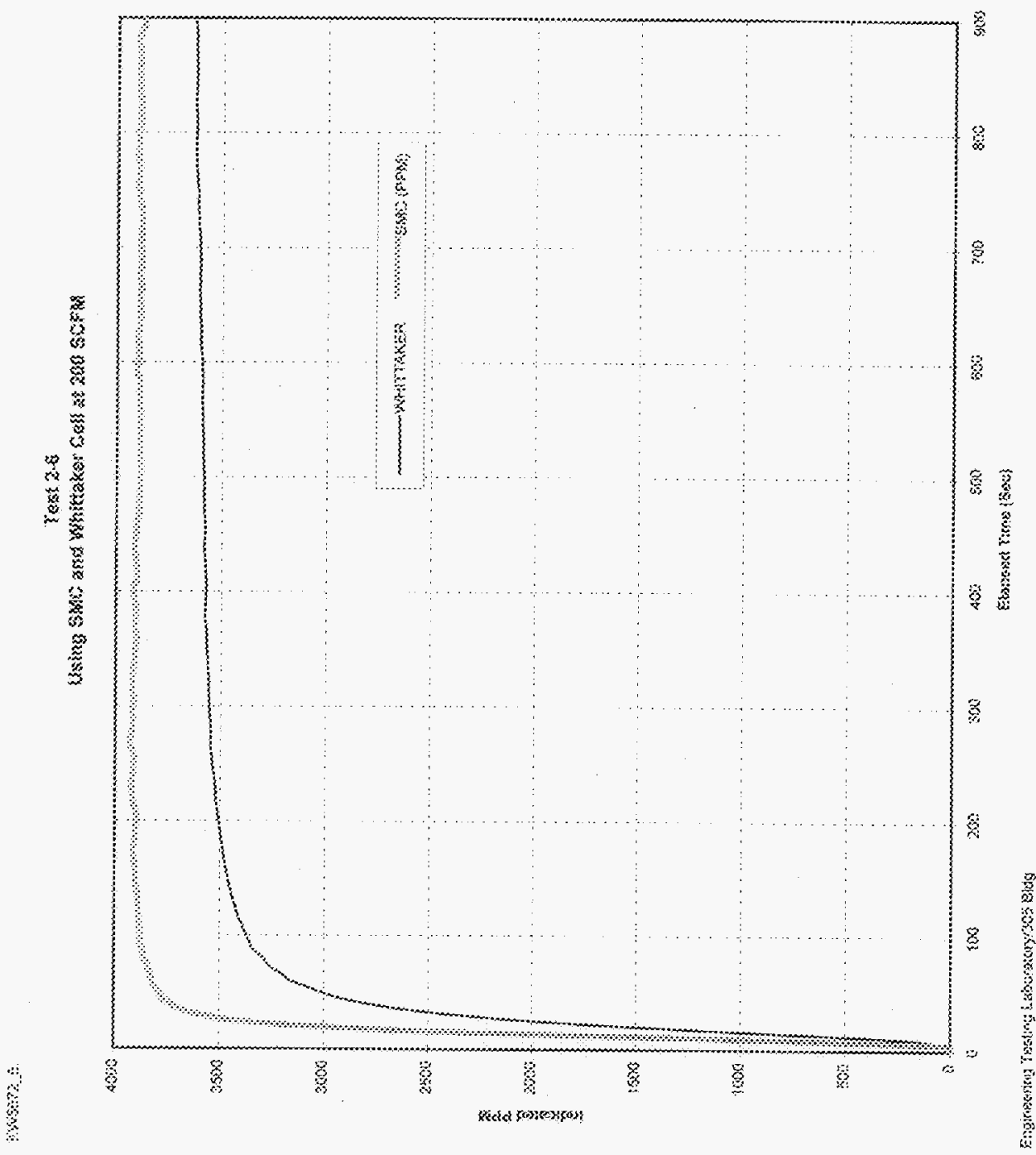




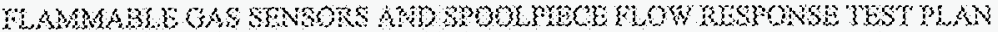
Axorkonousus:

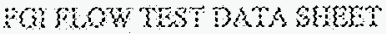

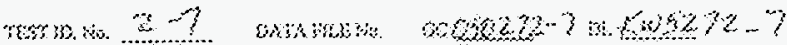

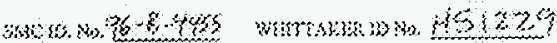

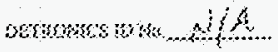

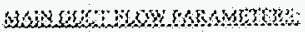

Howises) 200 oss

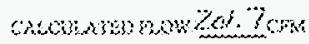

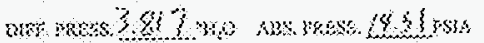

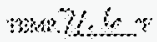

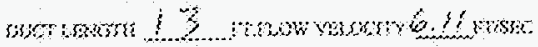

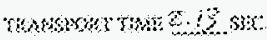

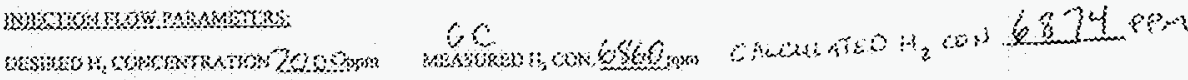

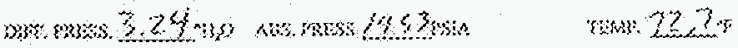

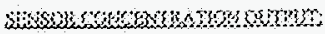

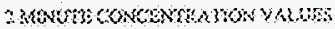

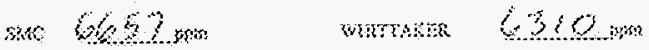

maswe $\{1, A \quad$ spa

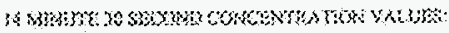

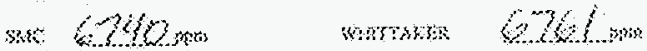

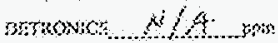

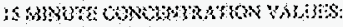

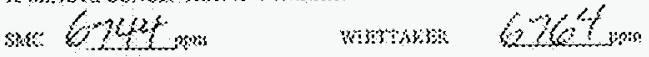

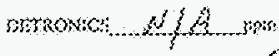

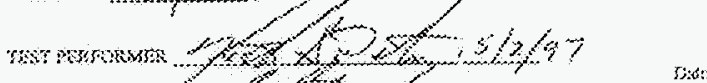

Thi bisctox:

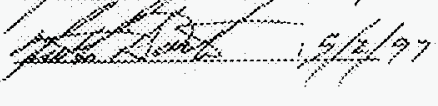

3ista 


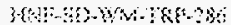

Box: :

"uses 4

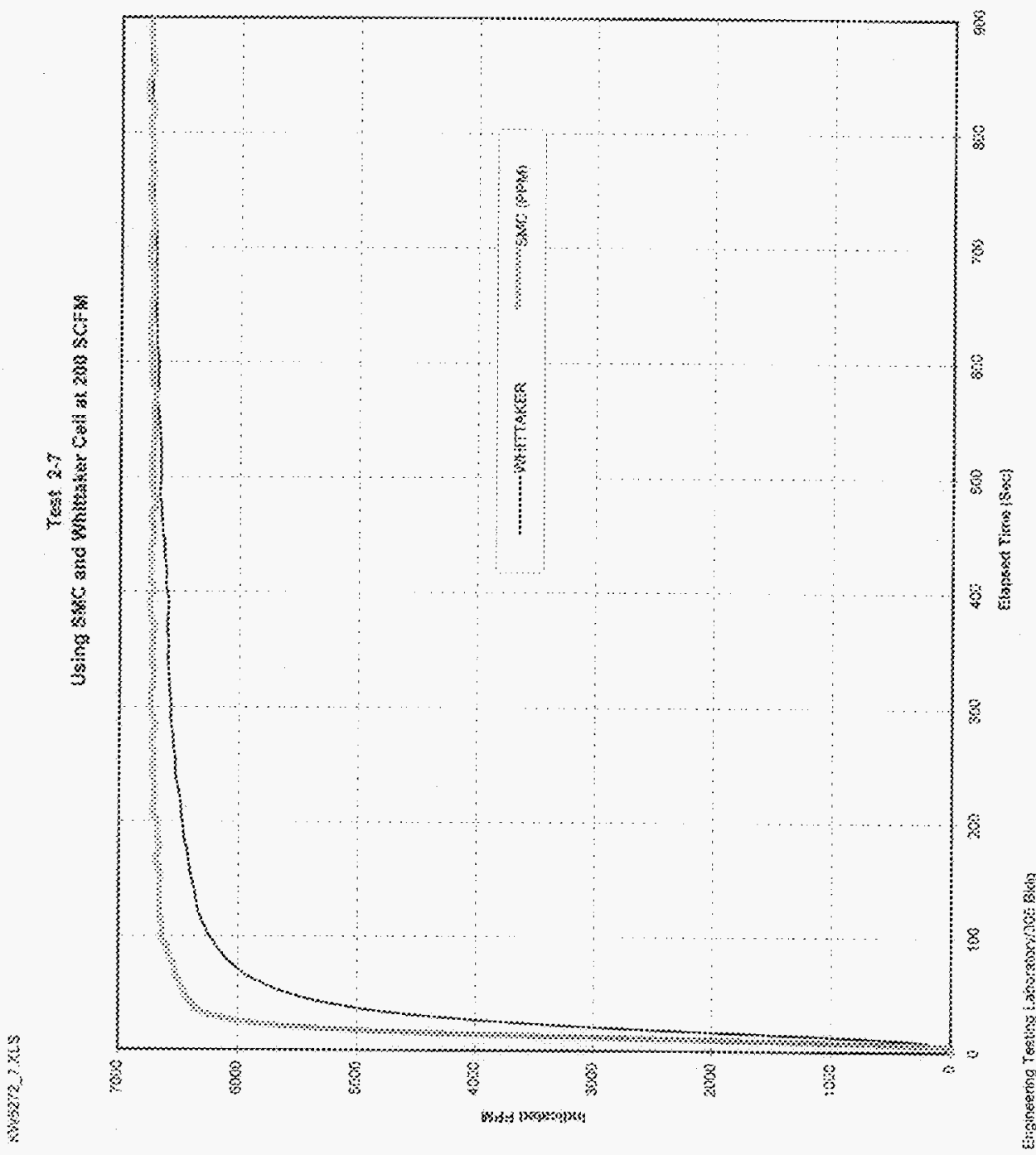




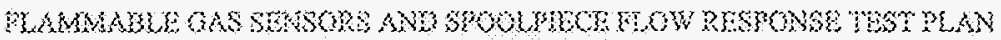

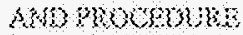

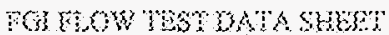

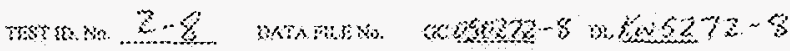

wow of-

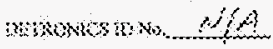

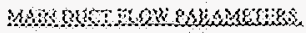

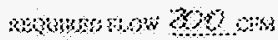

conn

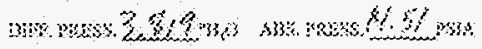
आas. $\%$

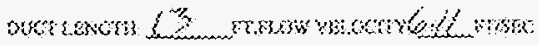

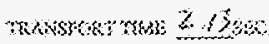

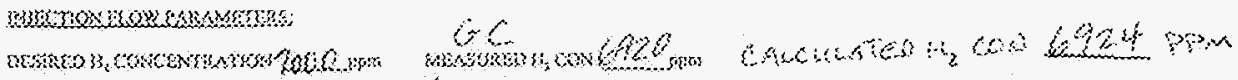

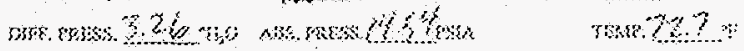

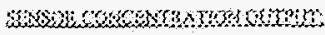

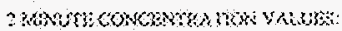

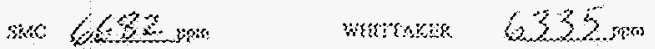

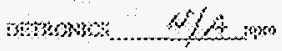

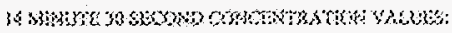

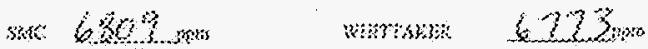

DRmows

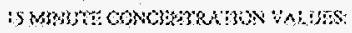

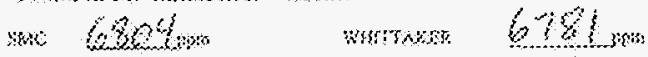

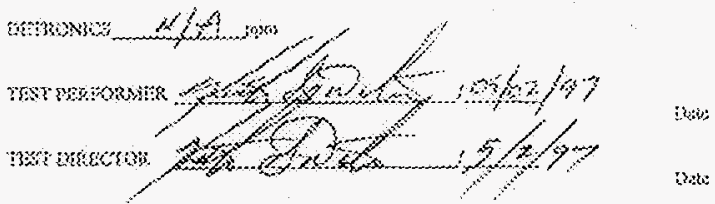




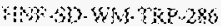

Rry os

Prgets

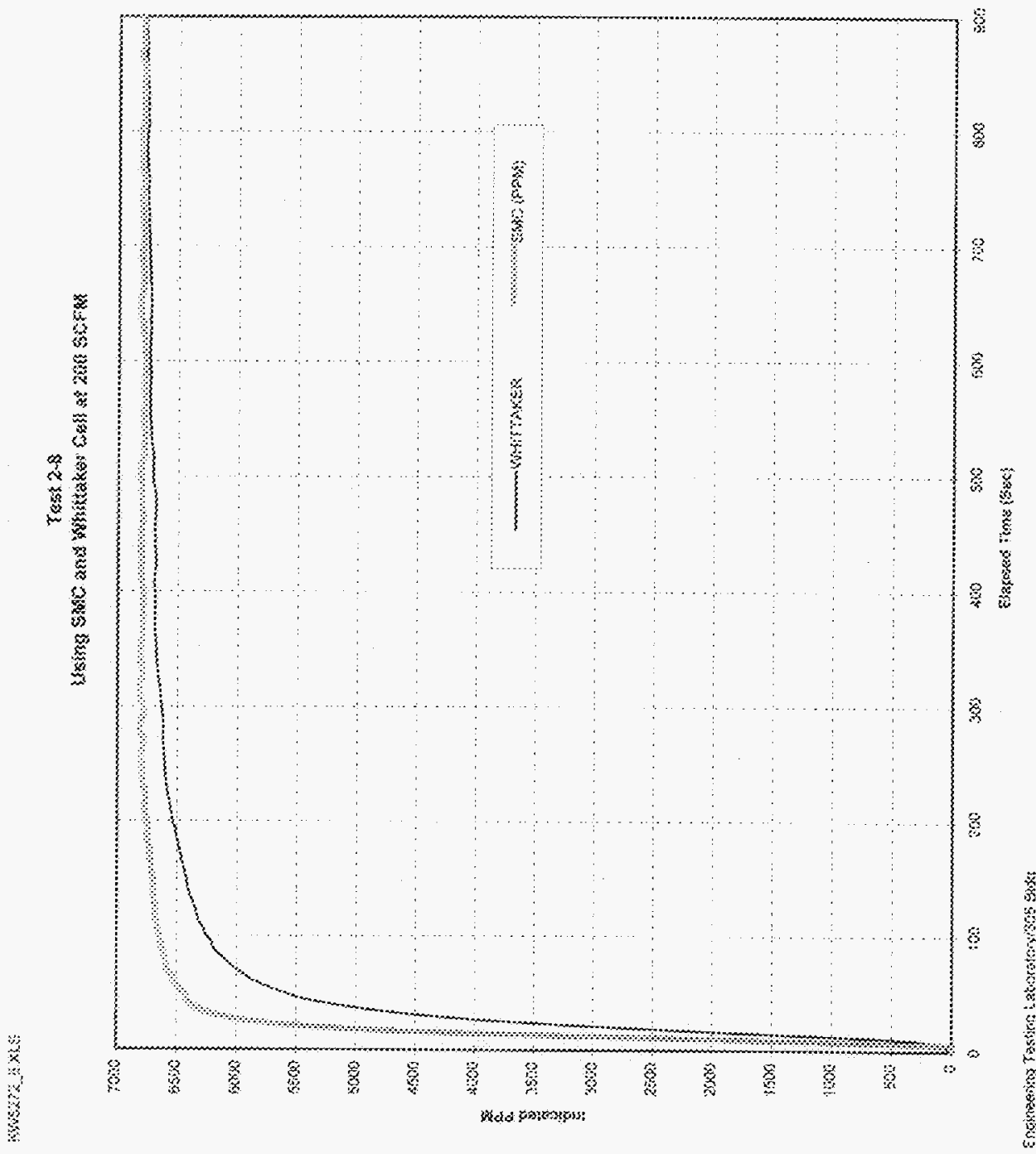




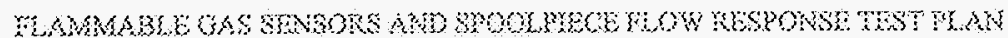
AND

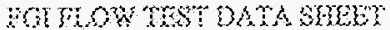

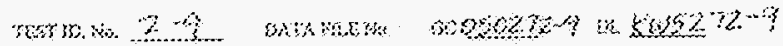

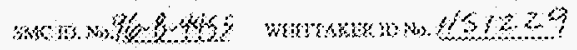

swowsober win

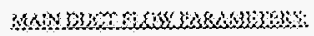

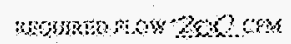
monnowow 2900

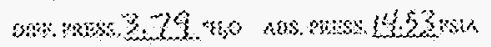
mot 72 ?

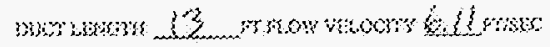

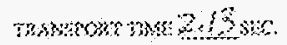

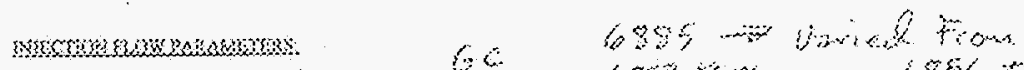

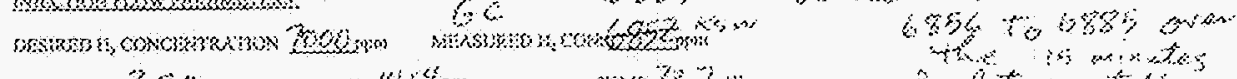

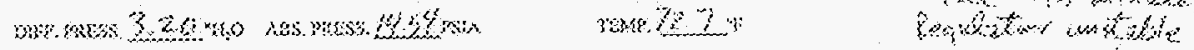

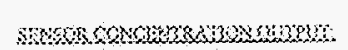

-. 3mbre rombrowin vinus:
sexc 3020
mank 62 s pon

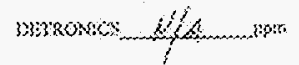

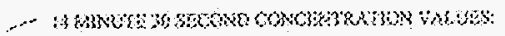
sic: $6 x+2 y$
whM
(a)

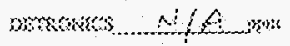

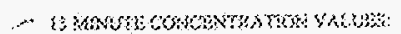

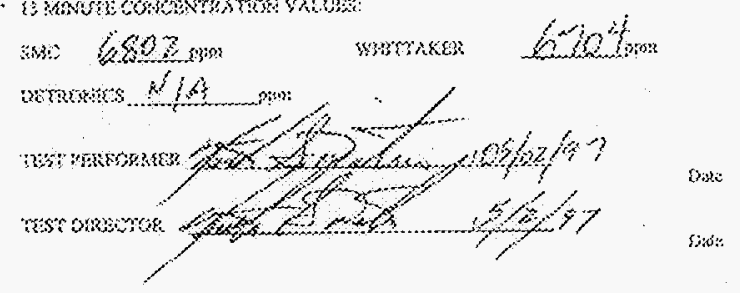


:

ist. 0

\{soc 4 ?

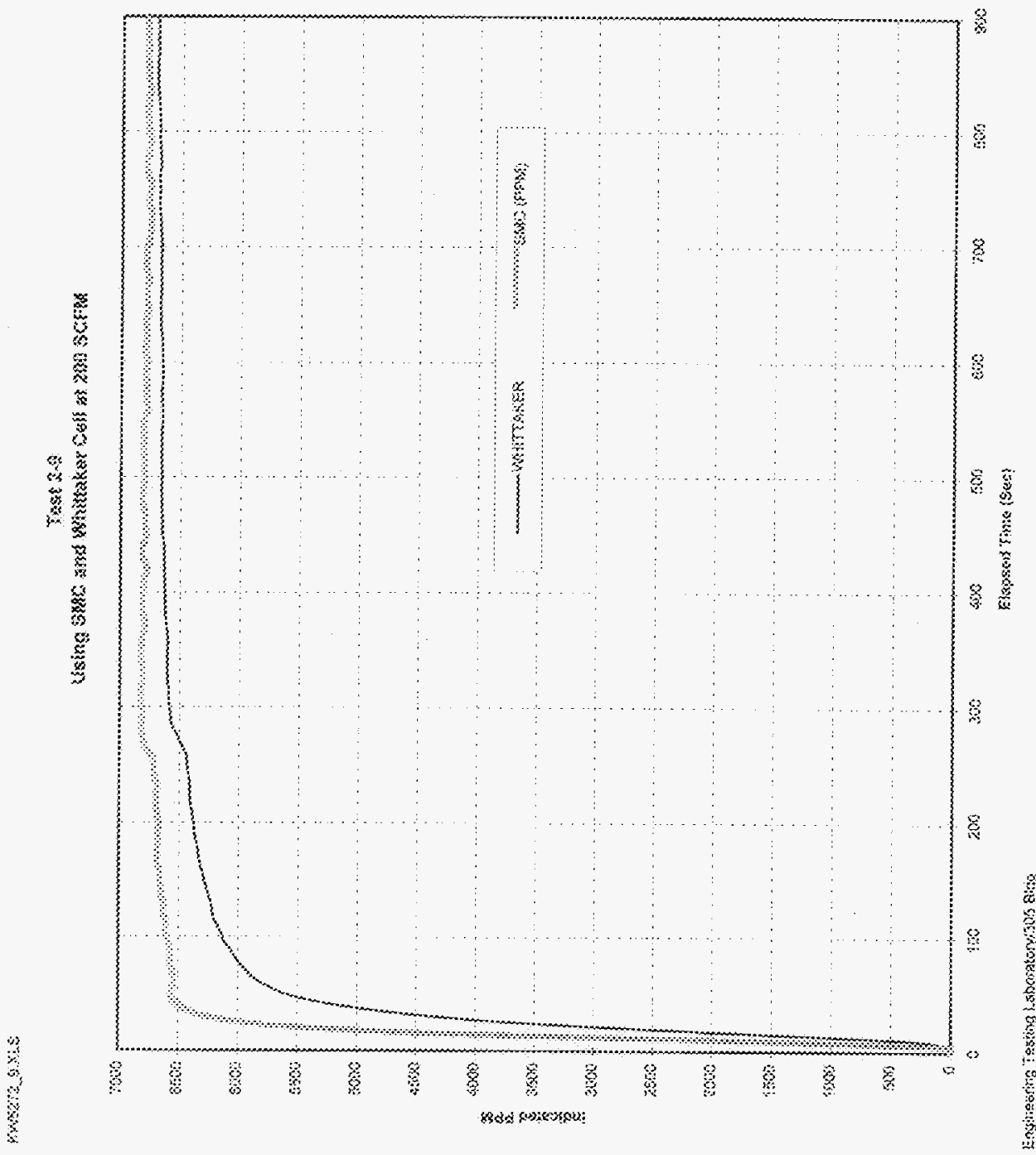



AND BQOCEWUX:

\section{EOOW WOW DATA SUEST}

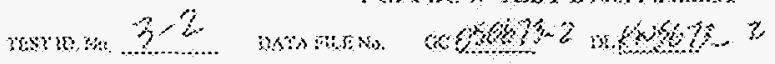

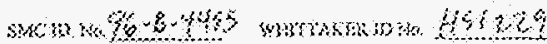

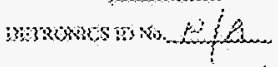

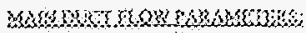

acoums rus 190 ow

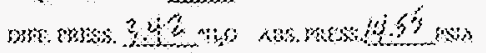

mosm

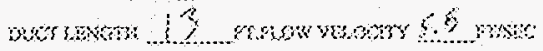

13:? ?:?

raxsos me? $36 \%$

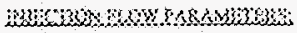

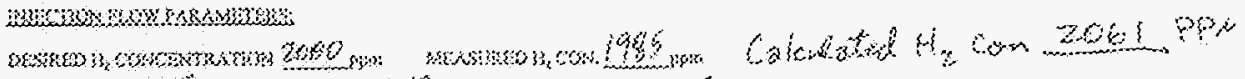

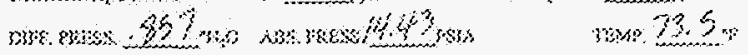

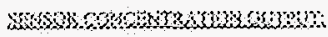

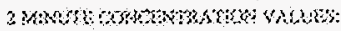

sж 790 momk

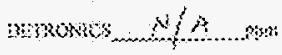

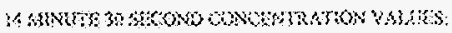

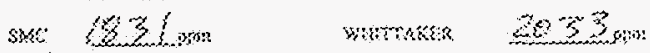

w)

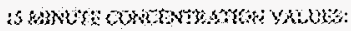

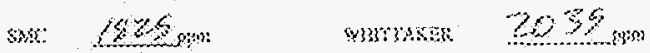

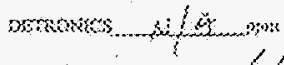

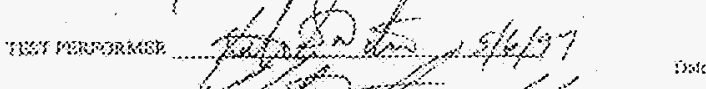

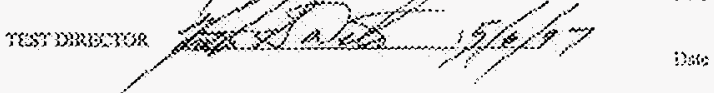




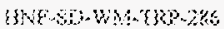

Qt:?

3 ing 40

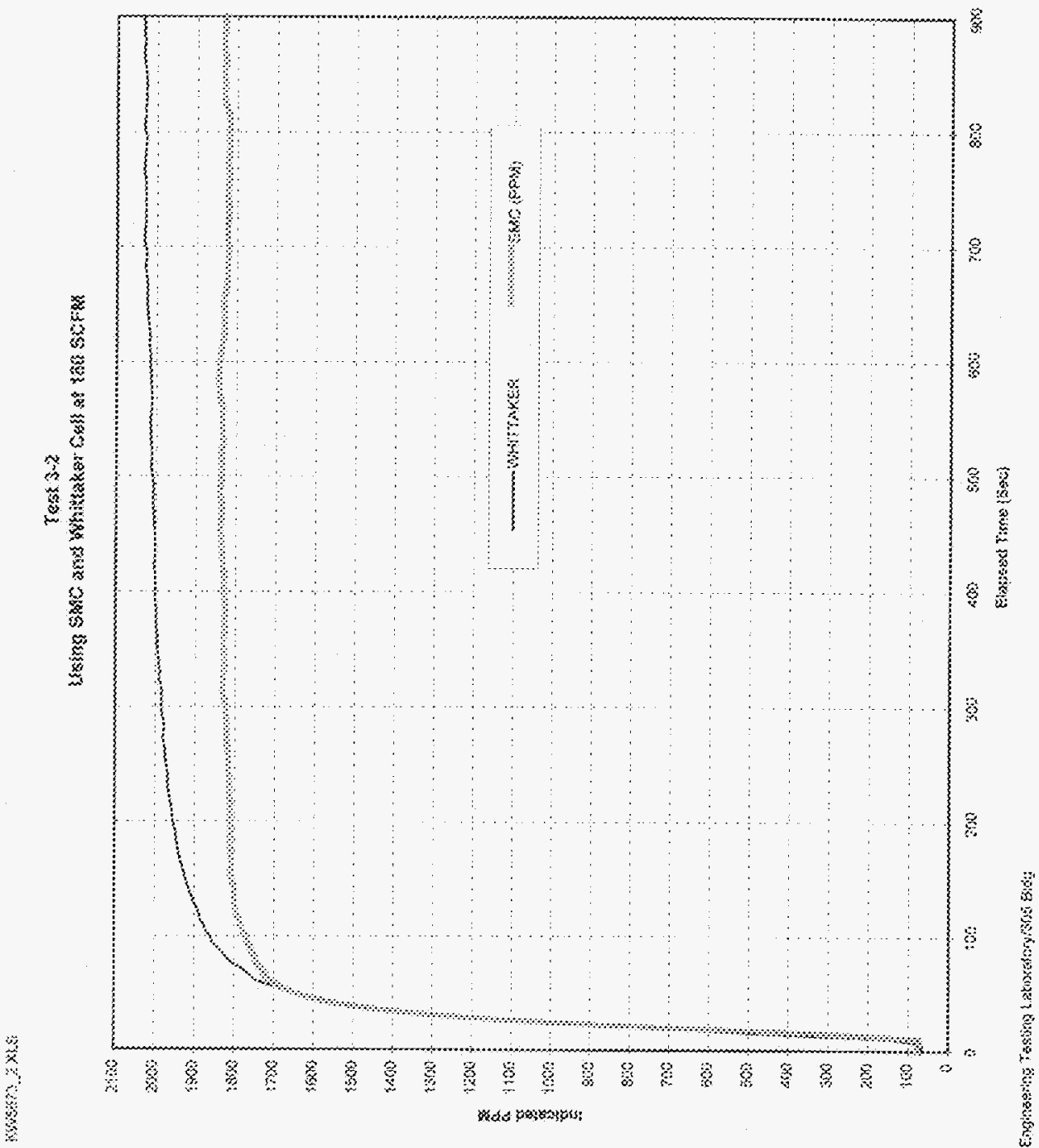




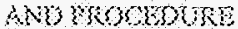

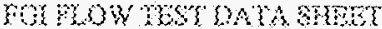

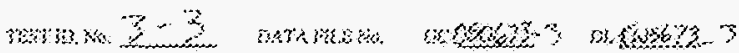

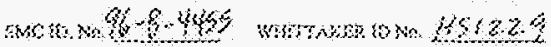

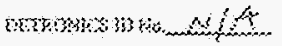

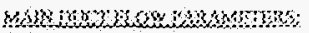

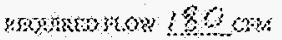

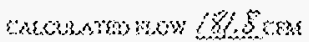

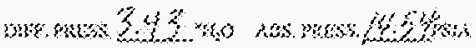

3022,22

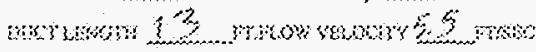

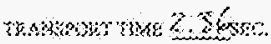

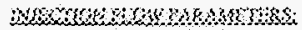

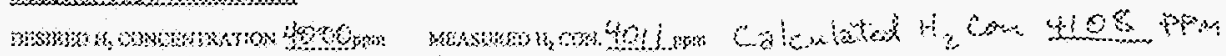

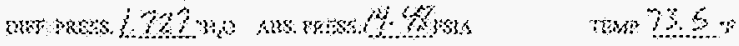

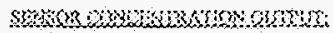

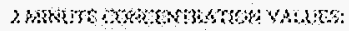

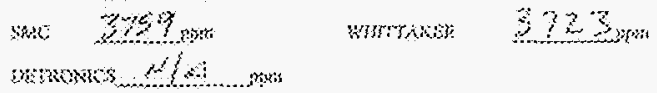

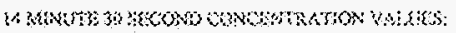

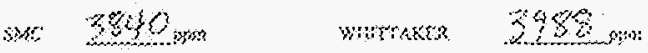

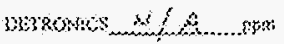

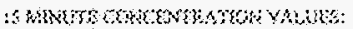

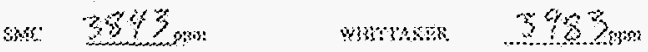

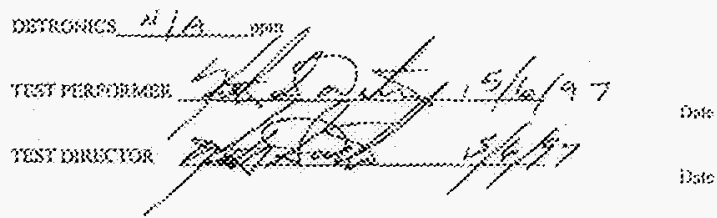




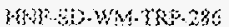

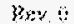

$3 \sin 5$ ?

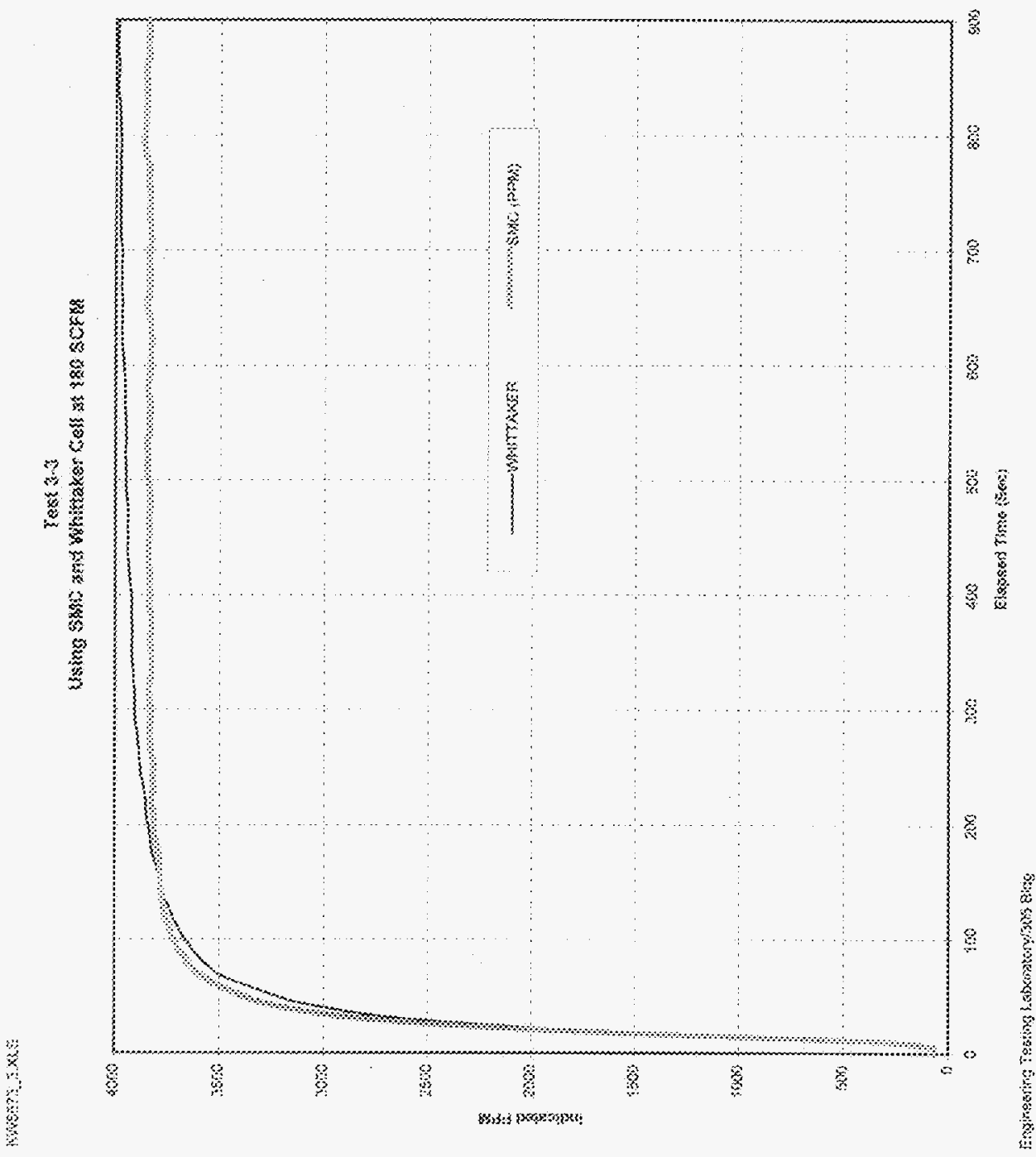




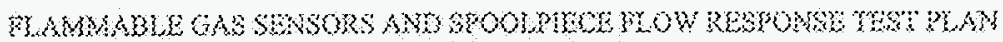

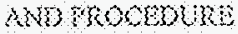

\section{WO WOW TWO WATA SIEET}

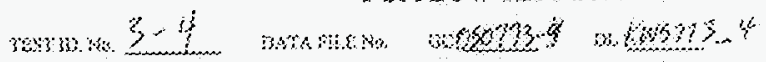

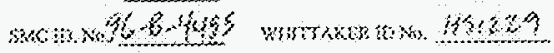

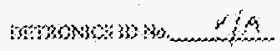

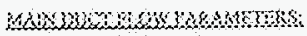
womonow 180 com

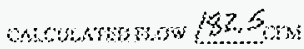

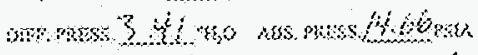
rensto. 7

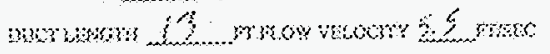

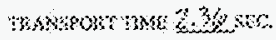

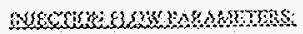

mан

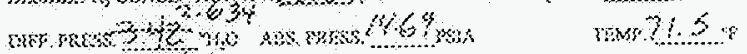
Xs...

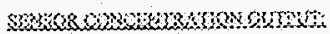

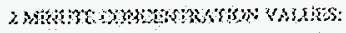

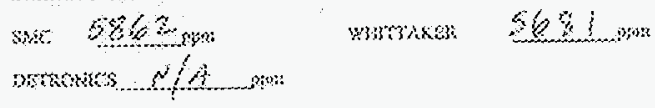

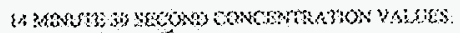

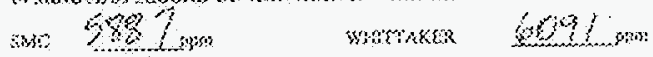

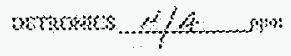

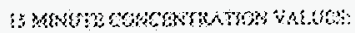

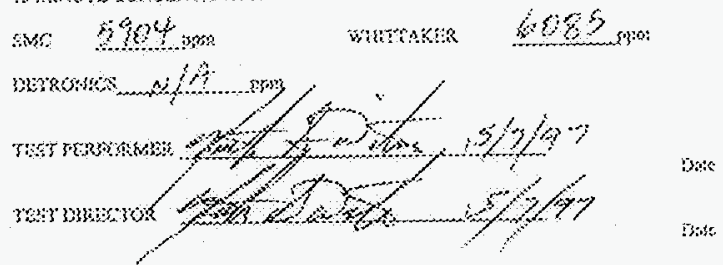




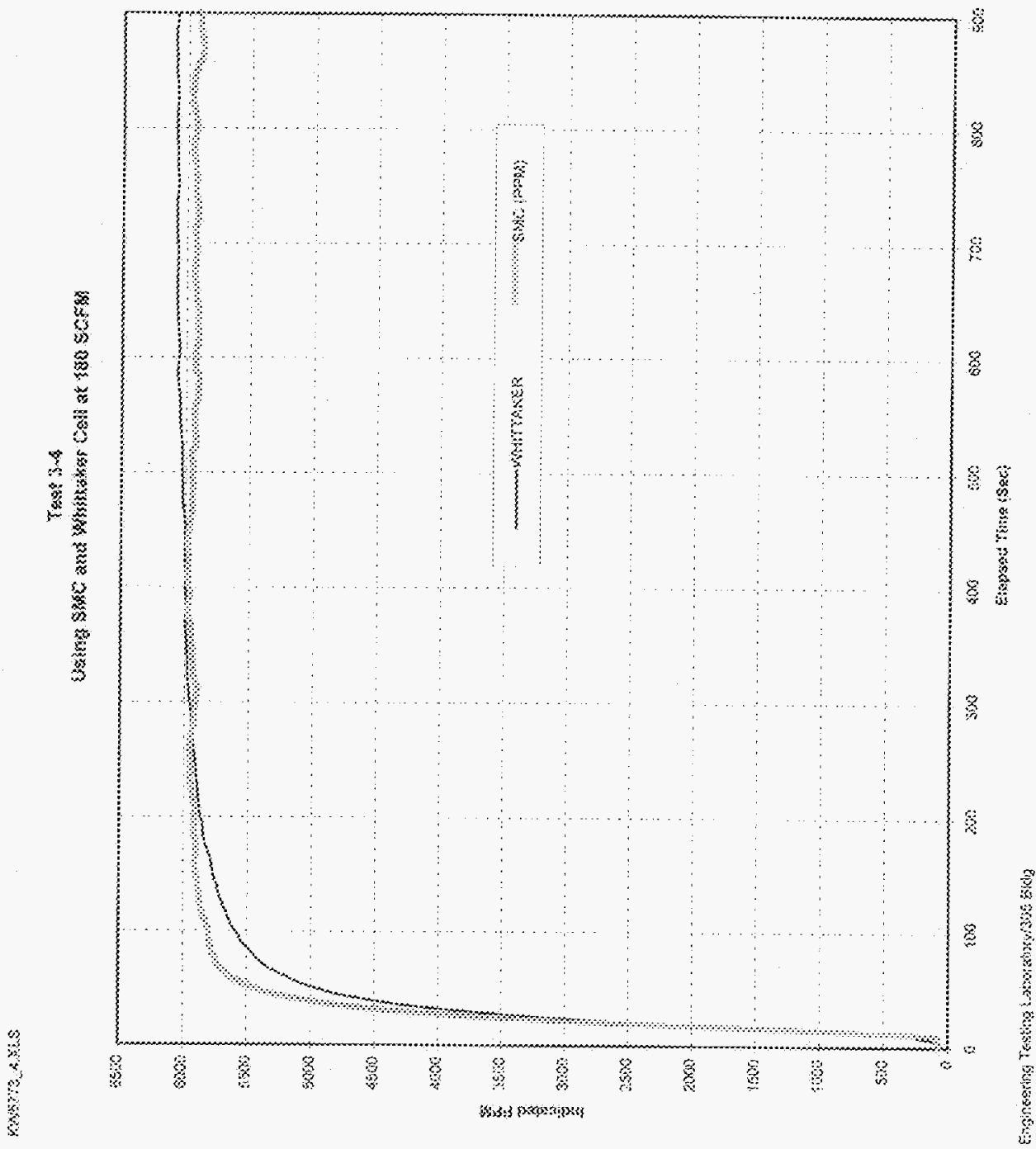




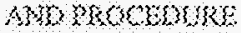

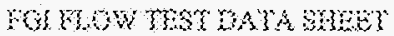

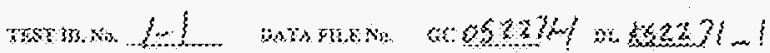

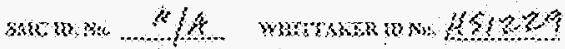

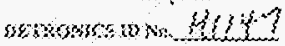

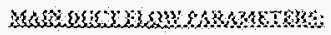

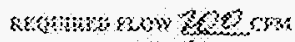

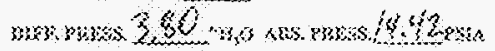

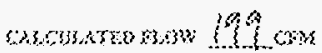
wat? 2 ?

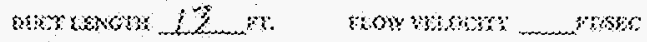

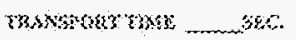

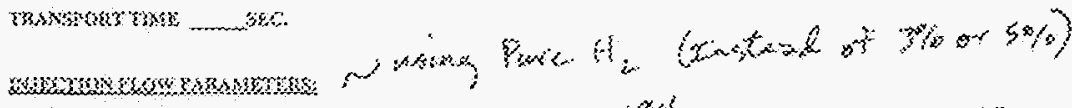

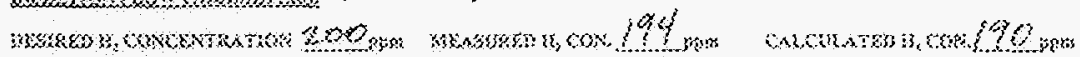

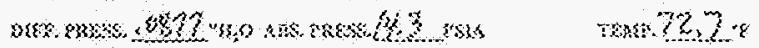

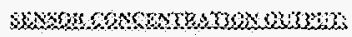

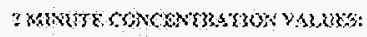

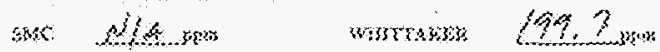

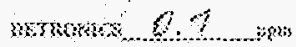

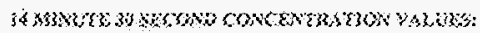

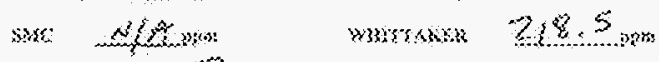

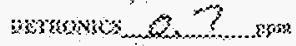

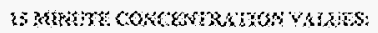

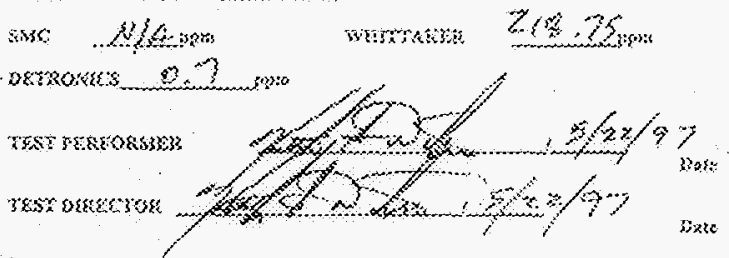




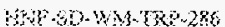

Buser. 0

Bge 58

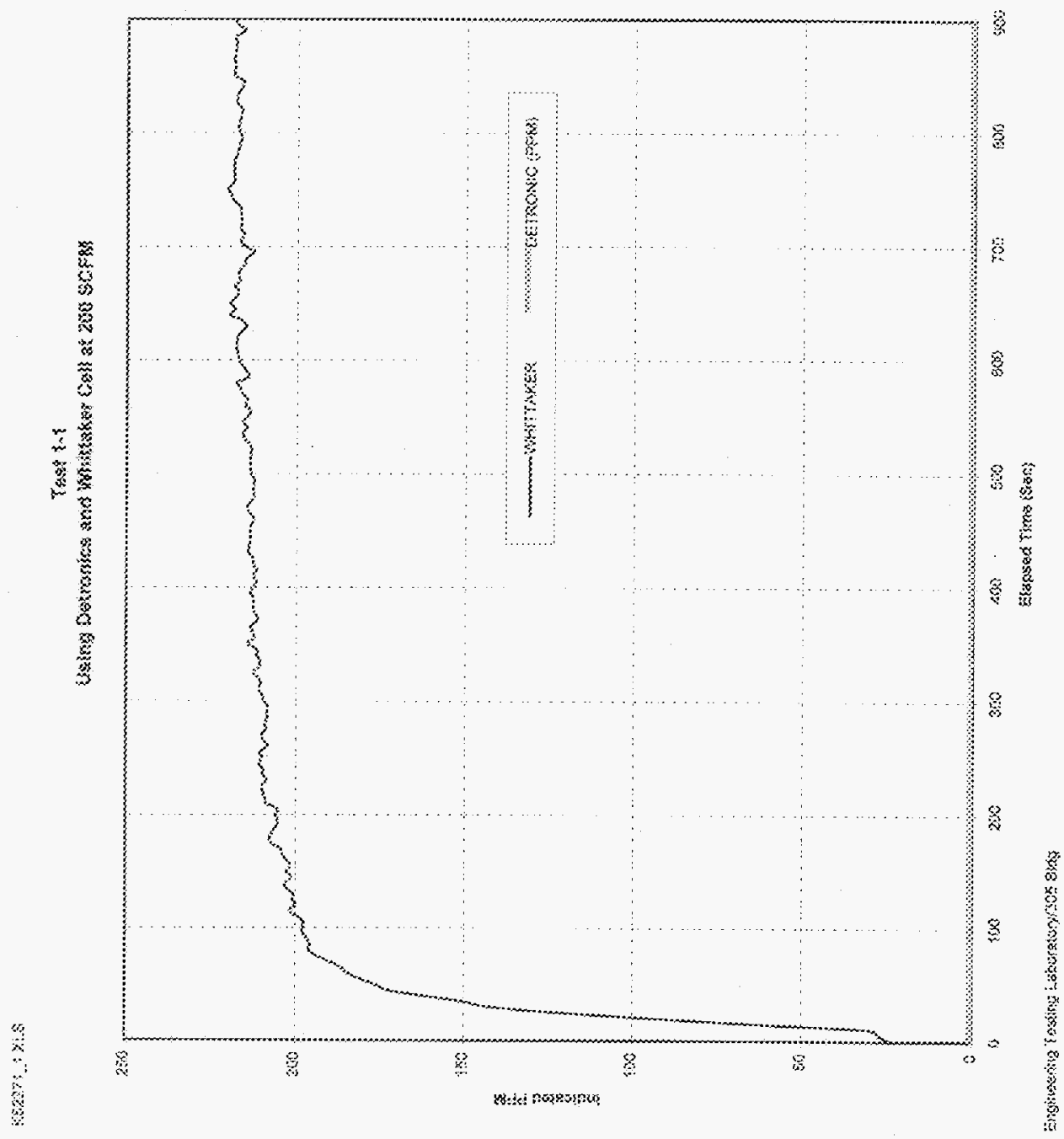




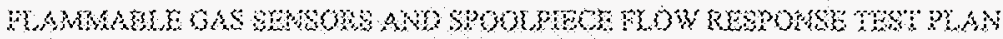
MWPWOWDSE

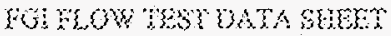

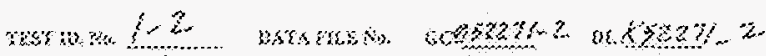

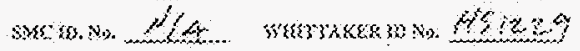

mixumberstio bify?

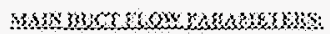

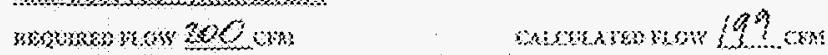

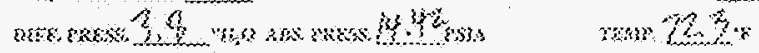

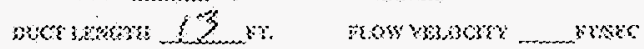

maks? $3 \times x \mathrm{max}$

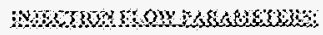

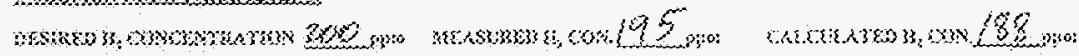
nwems 08 of

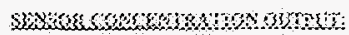

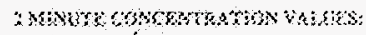

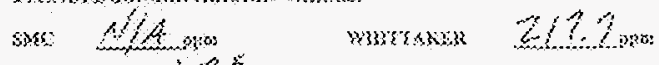

msxiskco $6 ? 2$

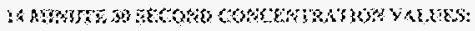

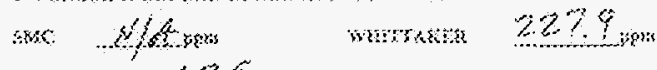

wrment $<2 \%$

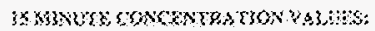

sme $\quad n / 20$ mon

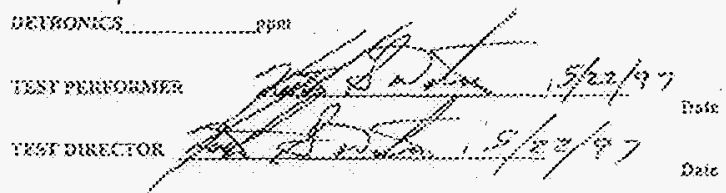




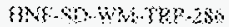

fiev. 0

fyos?

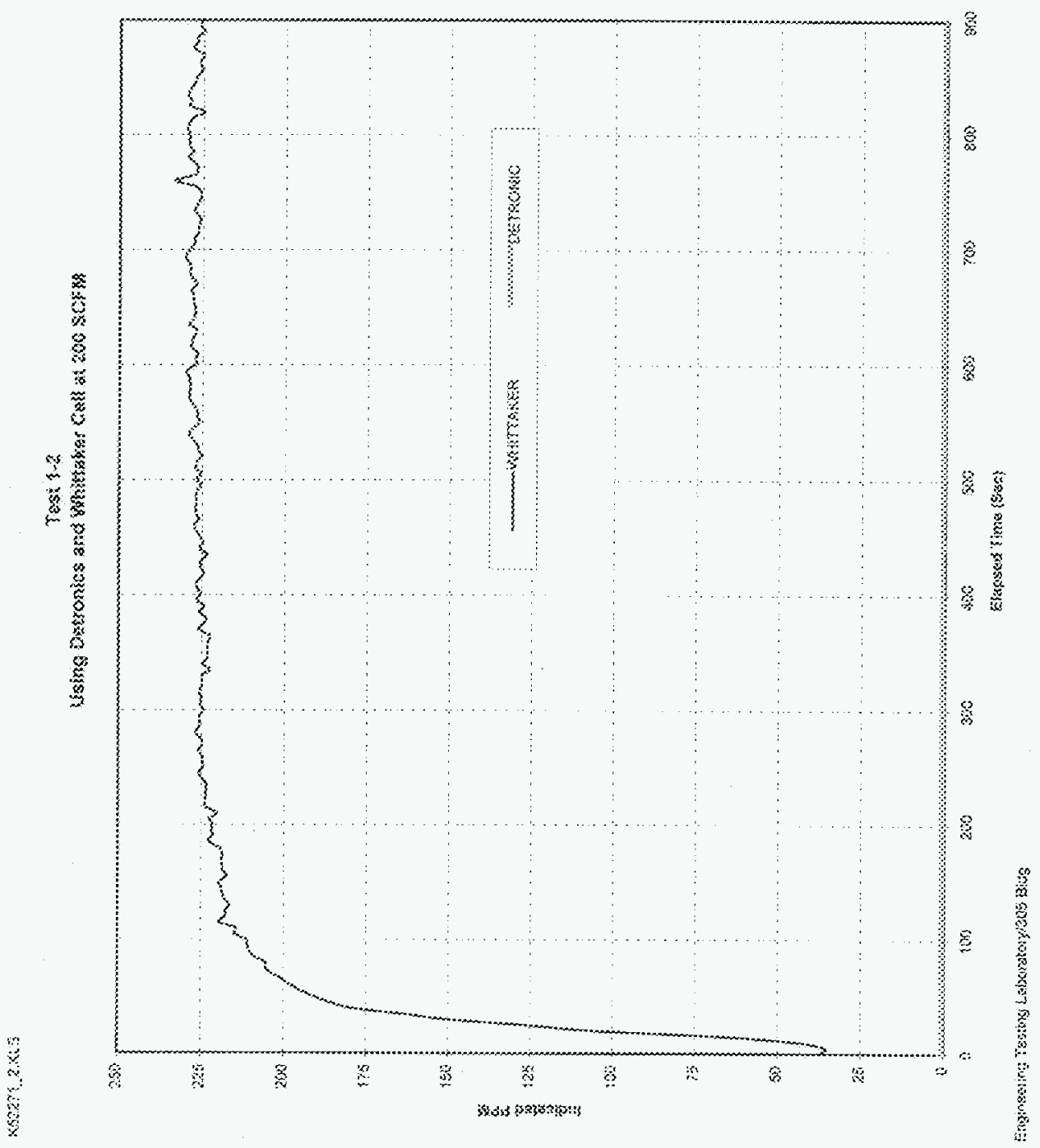




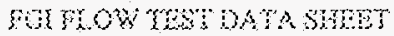

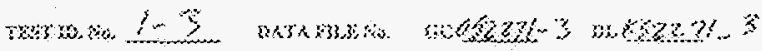

sms.

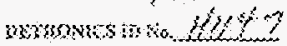

MSBWWCSCOW YBNAEYSSS

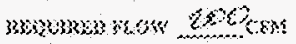

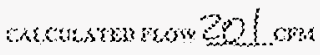

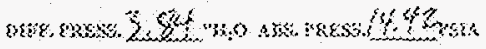

$38 \times 90.22 .6 .8$

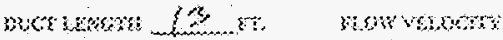
Hose

maksmom maxe sec.

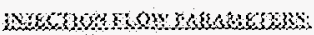

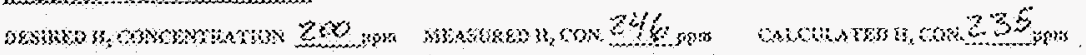

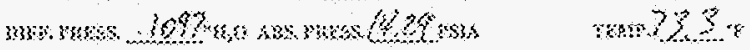

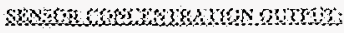

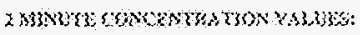

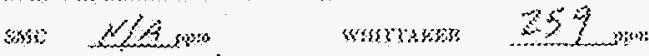

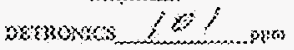

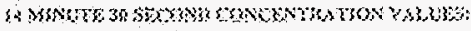

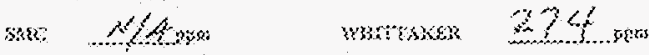

आR

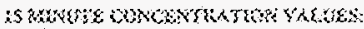

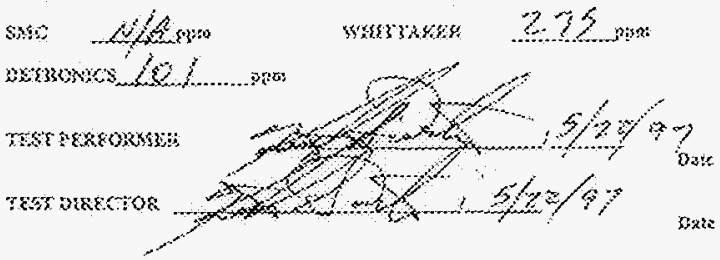




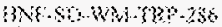

Ker.

\&rypes 59

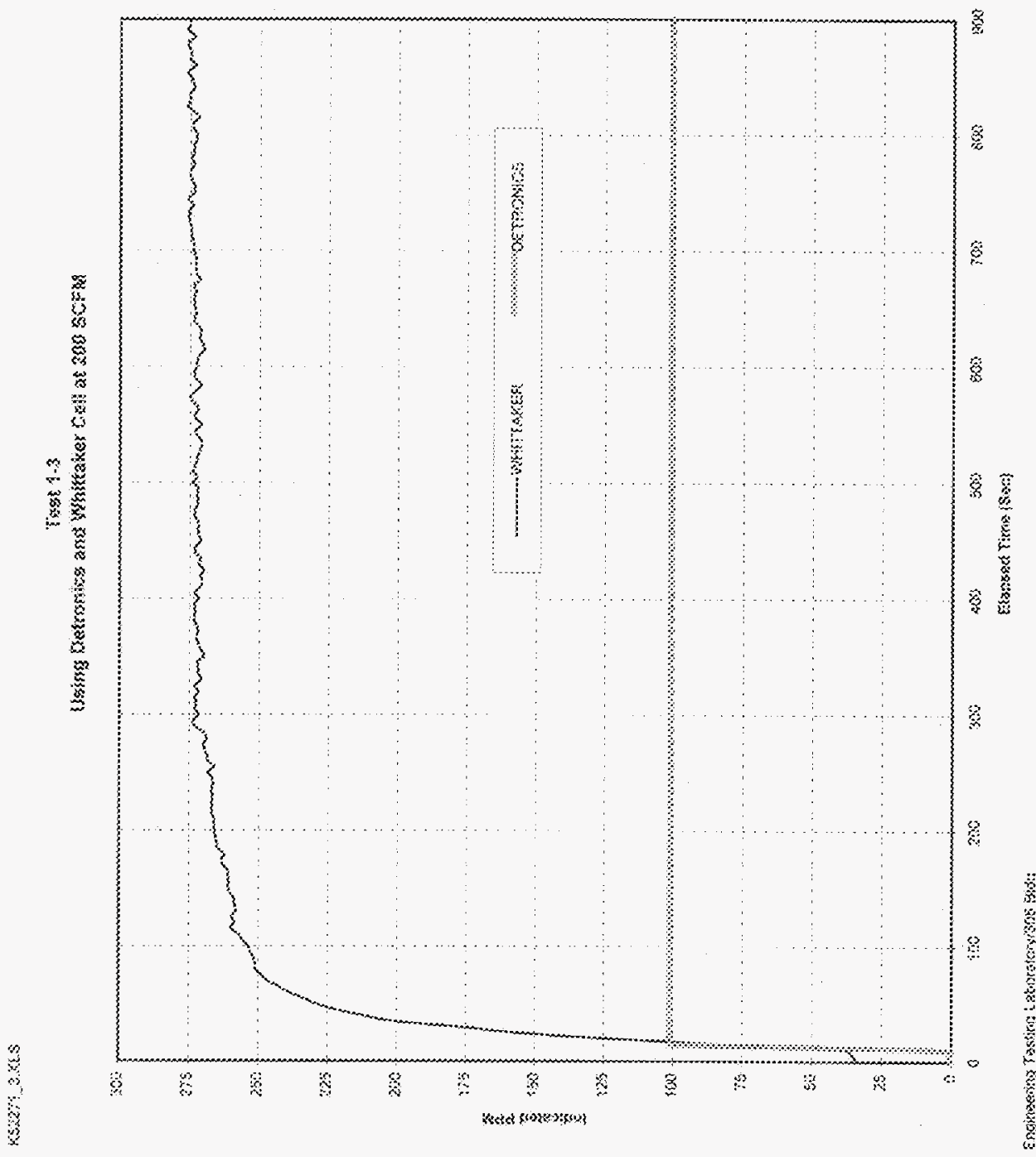




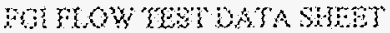

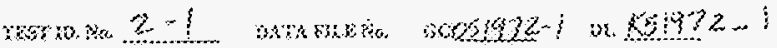

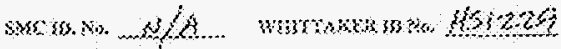

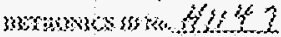

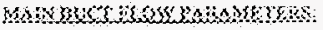

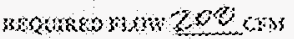

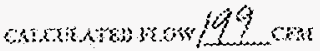

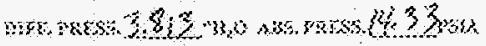

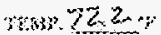

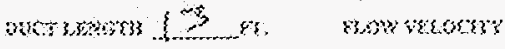
Fostix:

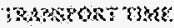
$5: 0$.

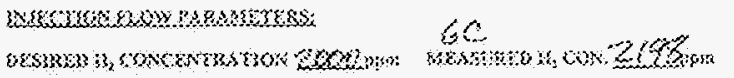

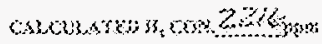

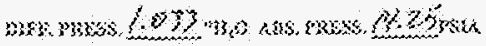

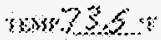

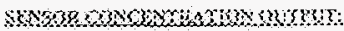

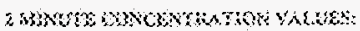

Hisc $4 / 4$ mom

orsmores $z$ inesp

is now

sso $\quad x / 4$ son

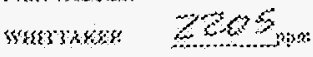

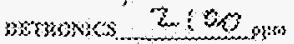

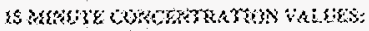

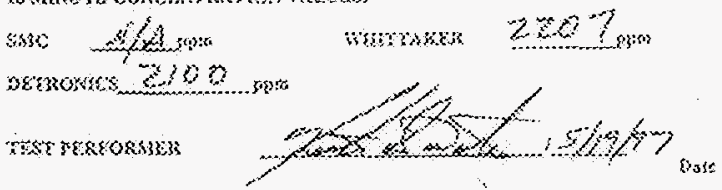

Throtrecon 


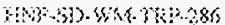

Bus

3ate 61

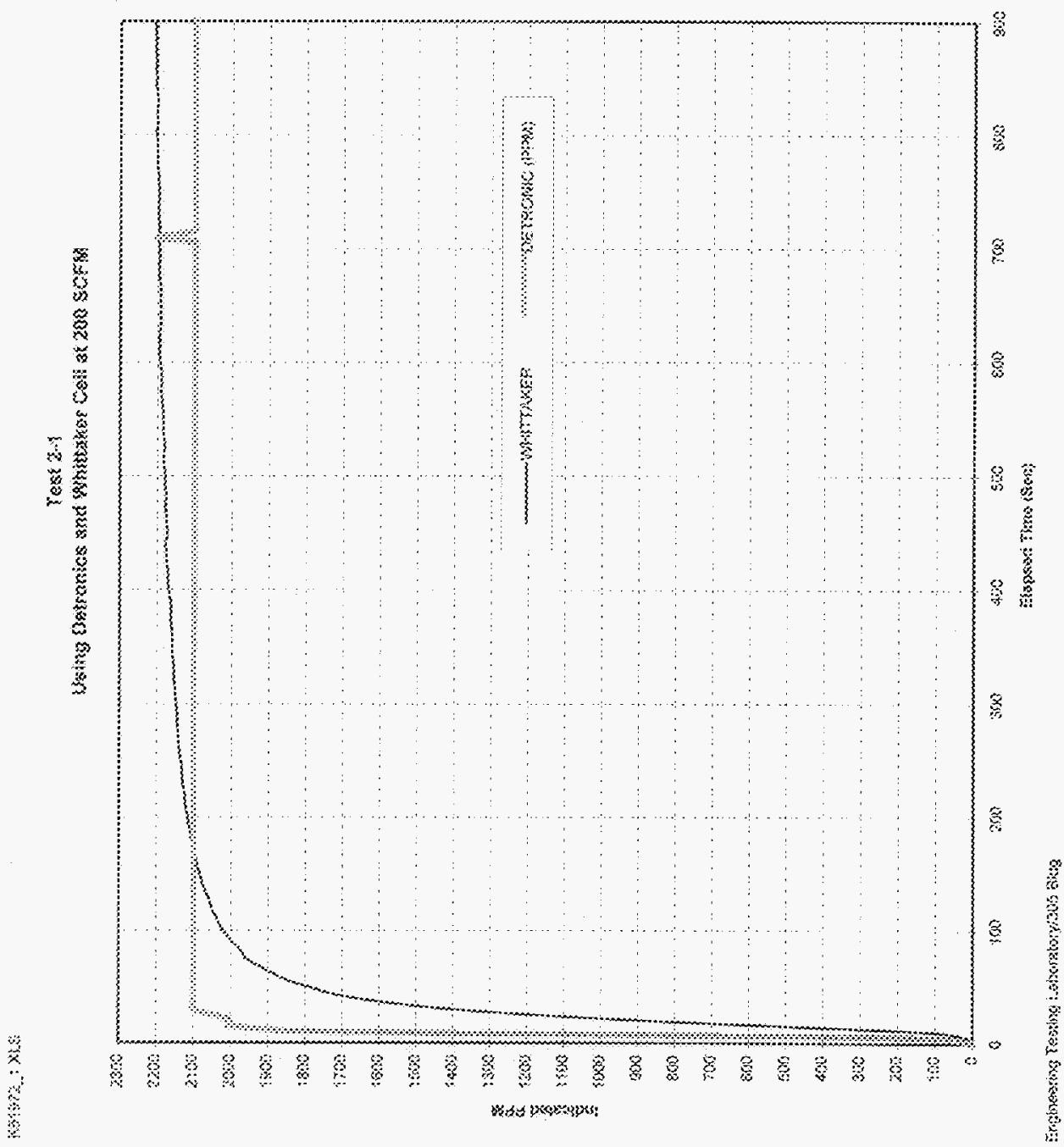




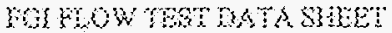

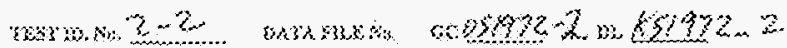

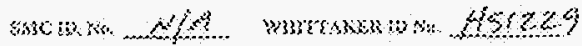

osmoxhs wh KIS?

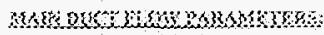

reoumunow 20\%

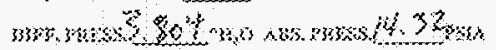

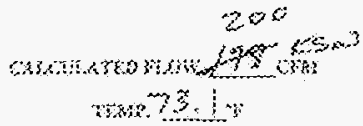

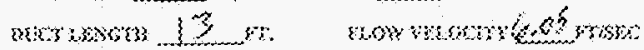

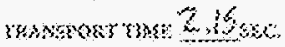

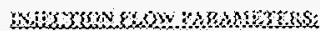

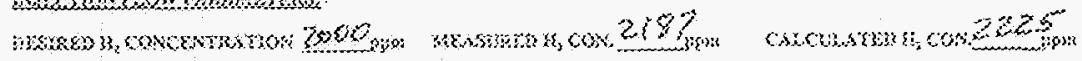

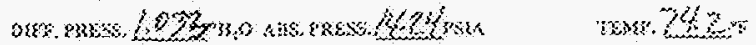

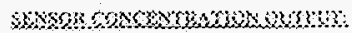

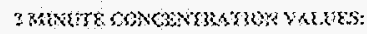

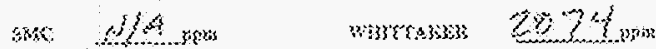

mruowites $10 \%$

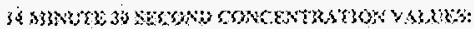

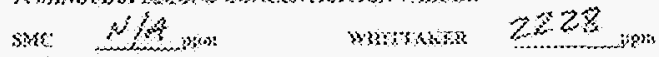

axmsus 200

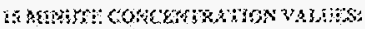

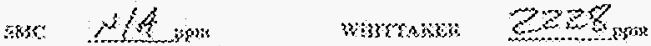

berkotiss 2,0 ,

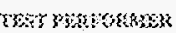

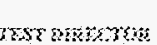

ings

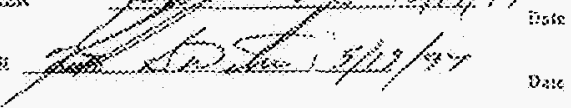




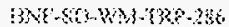

sed: 0

ferot 3

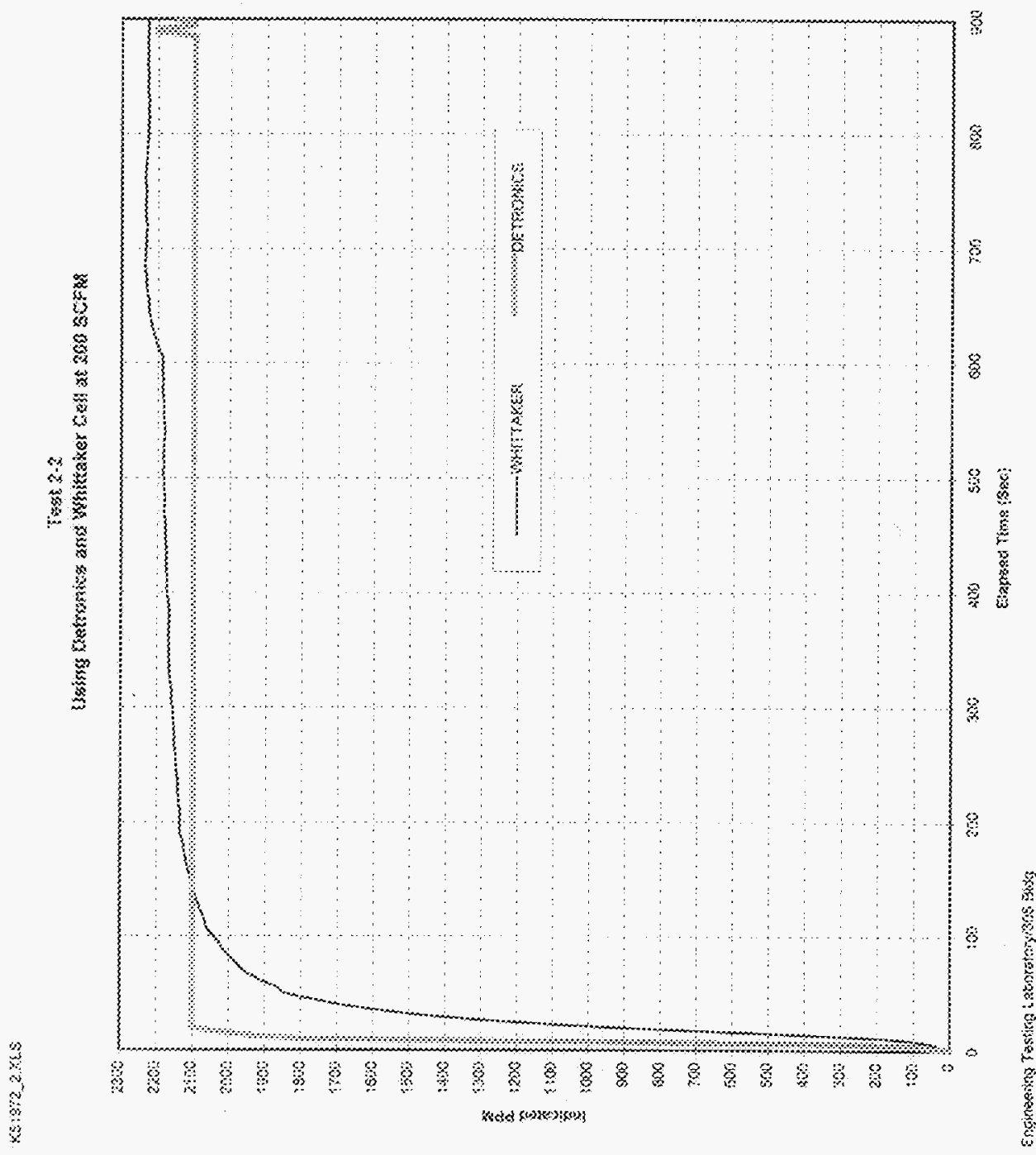




\section{RG MOW WESTATA SYYET}

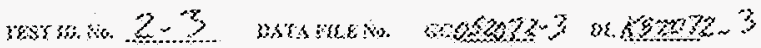

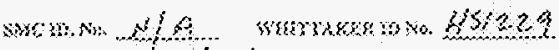

moswom

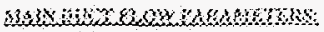

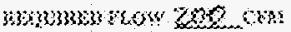

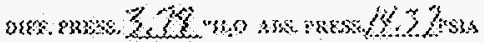

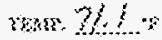

3)

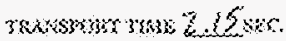

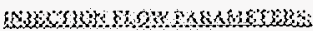

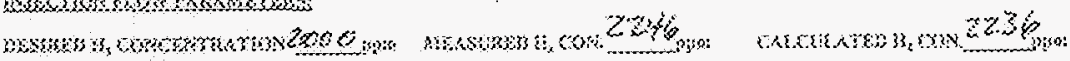

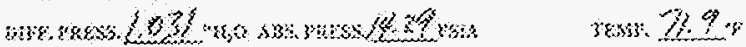

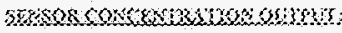

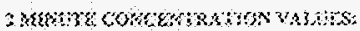

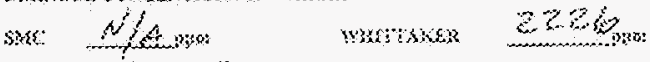

manksas 21050

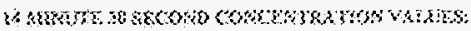

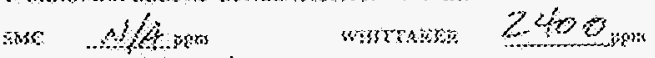

whrowes $220 !$

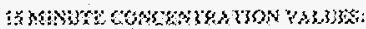

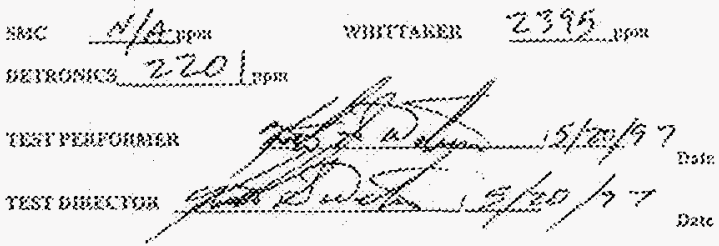




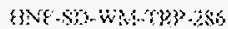

(A) 0

(4) 65

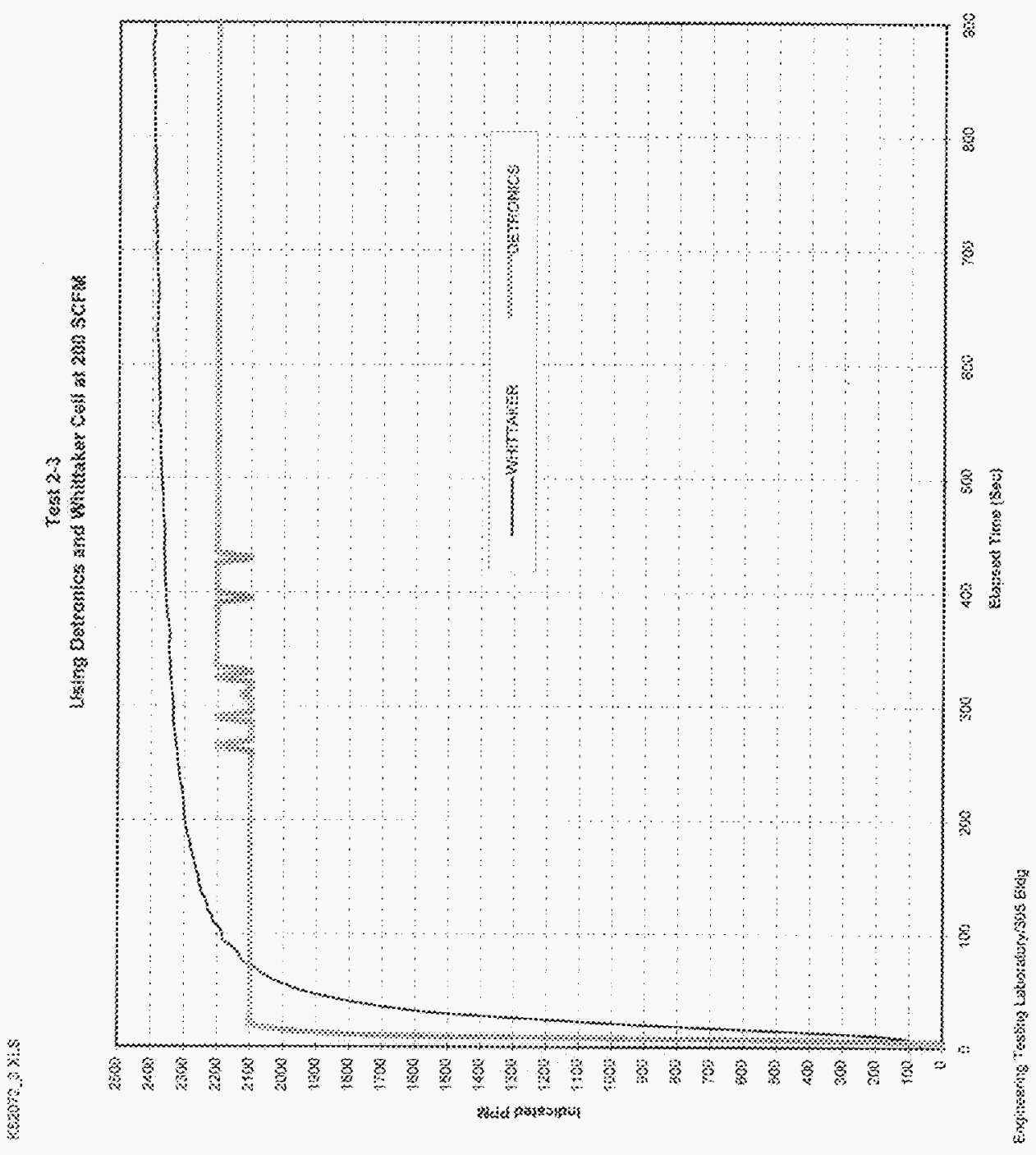




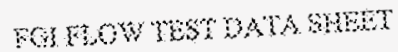

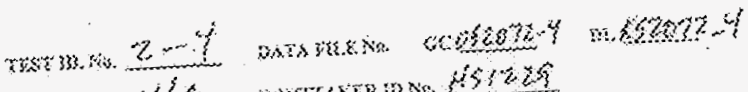

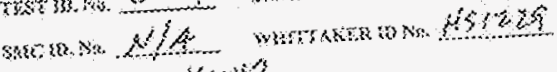

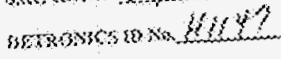

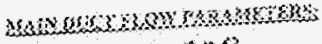

arawow row 200 on

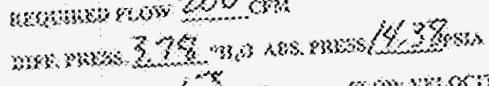

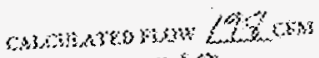

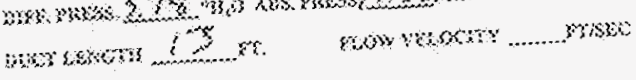

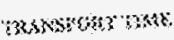
swo

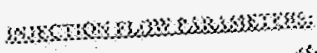

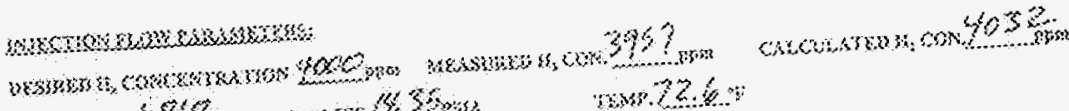

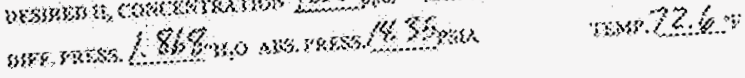

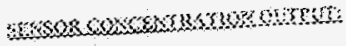

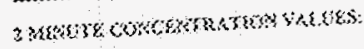

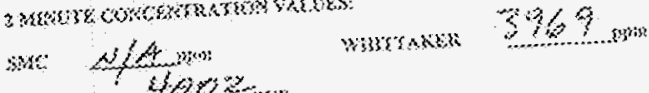

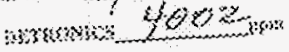

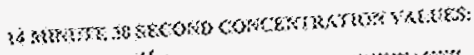

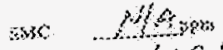

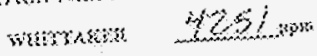

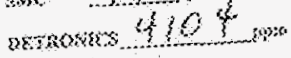

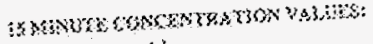

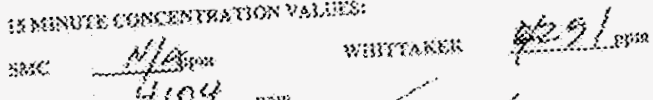

msabict 204 ....

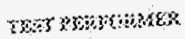

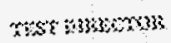

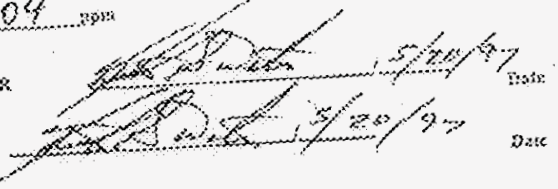




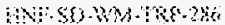

:isy 6

anes 67

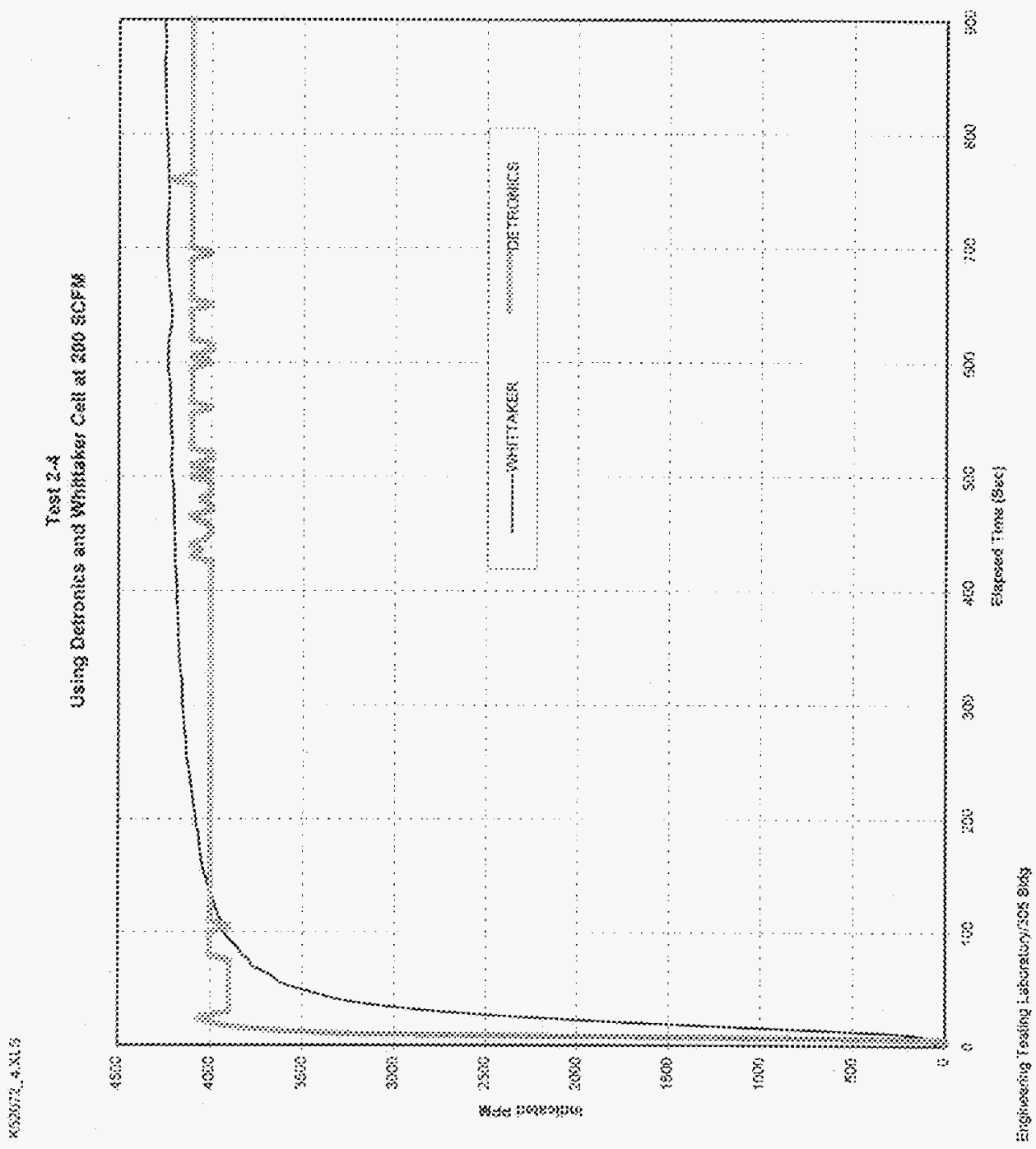




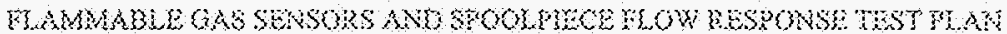

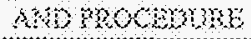

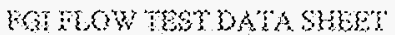

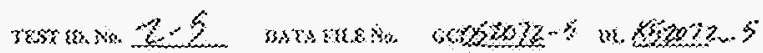

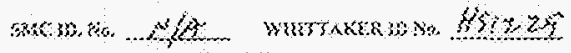

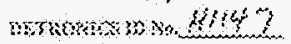

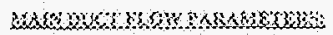

neowresson 200 ons

w3w. whss 318 .

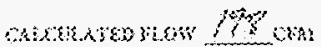
resporis 9

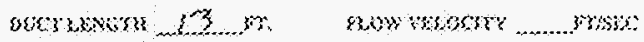

risaxporstrws जิ:

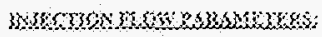

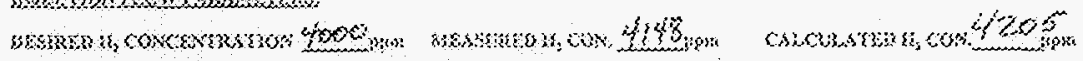

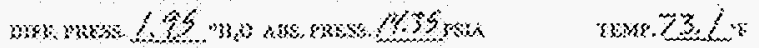

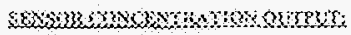

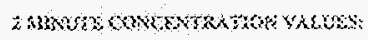

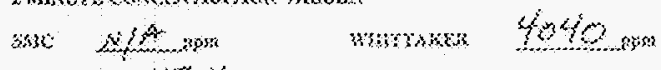

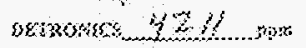

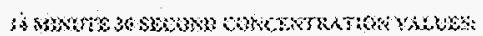

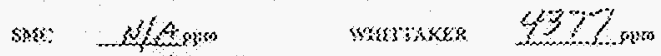

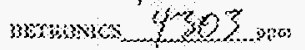

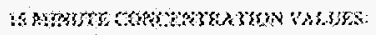

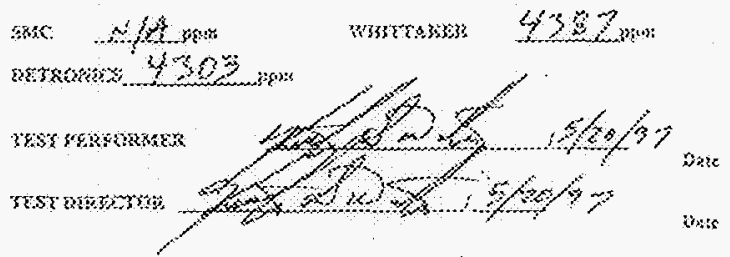




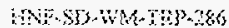

Kev. 0

\%oge $x$

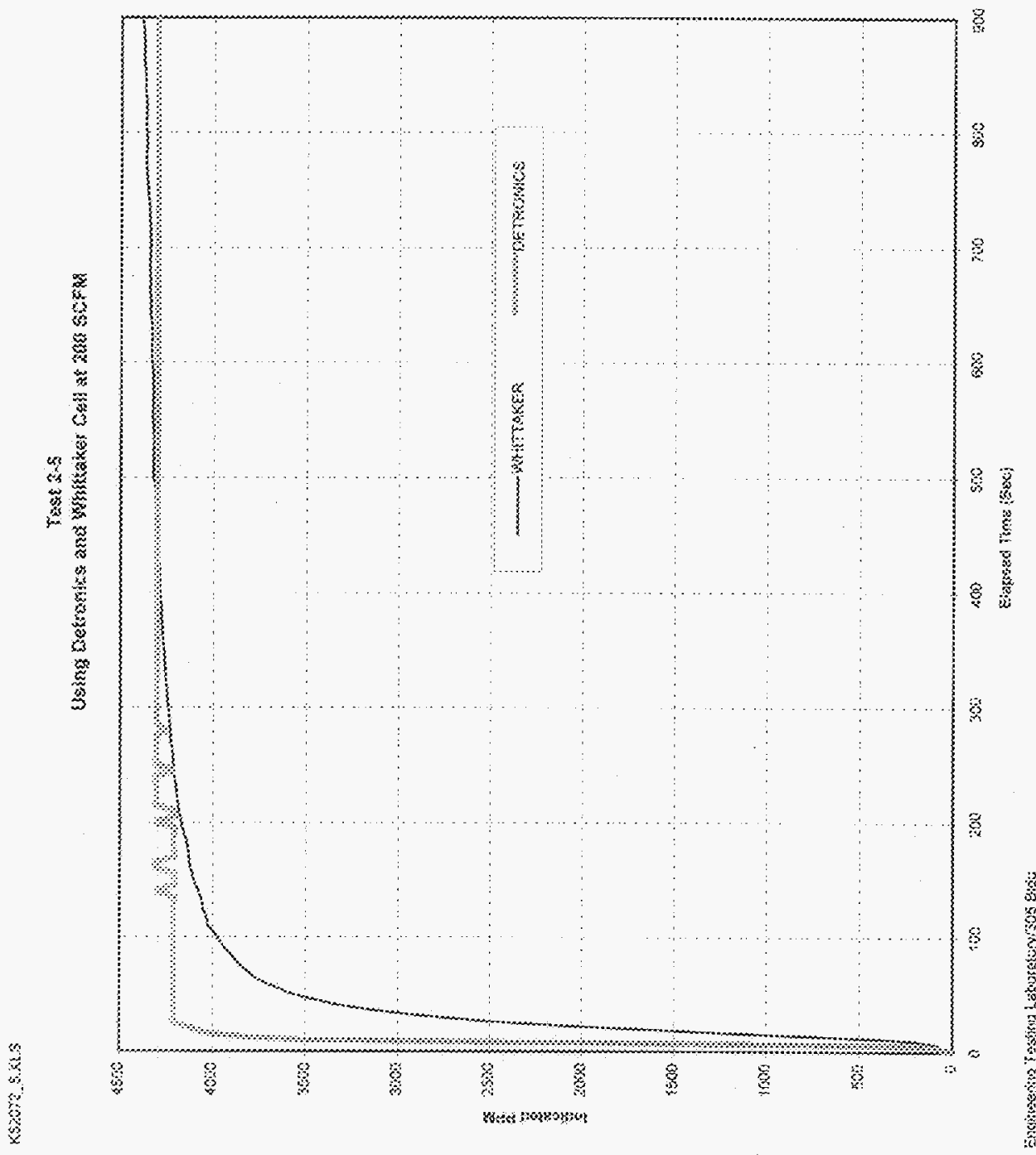




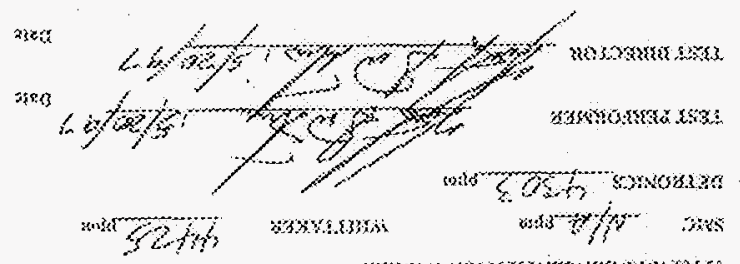

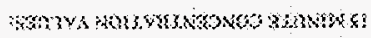

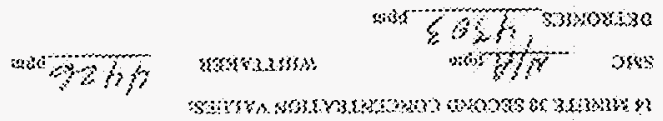

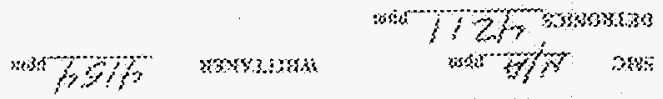

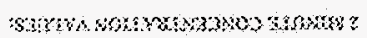
Mumpramplownoms

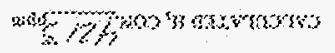

$232 \mathrm{ama}$ "20.6\%

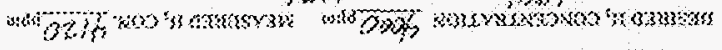

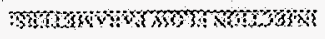

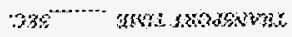

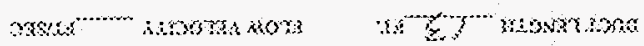

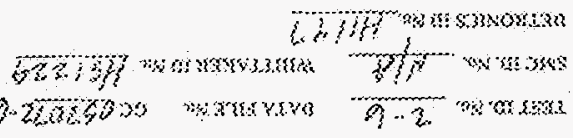

Mems verabu nom 


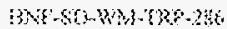

\{ex: 6

[rog ?]

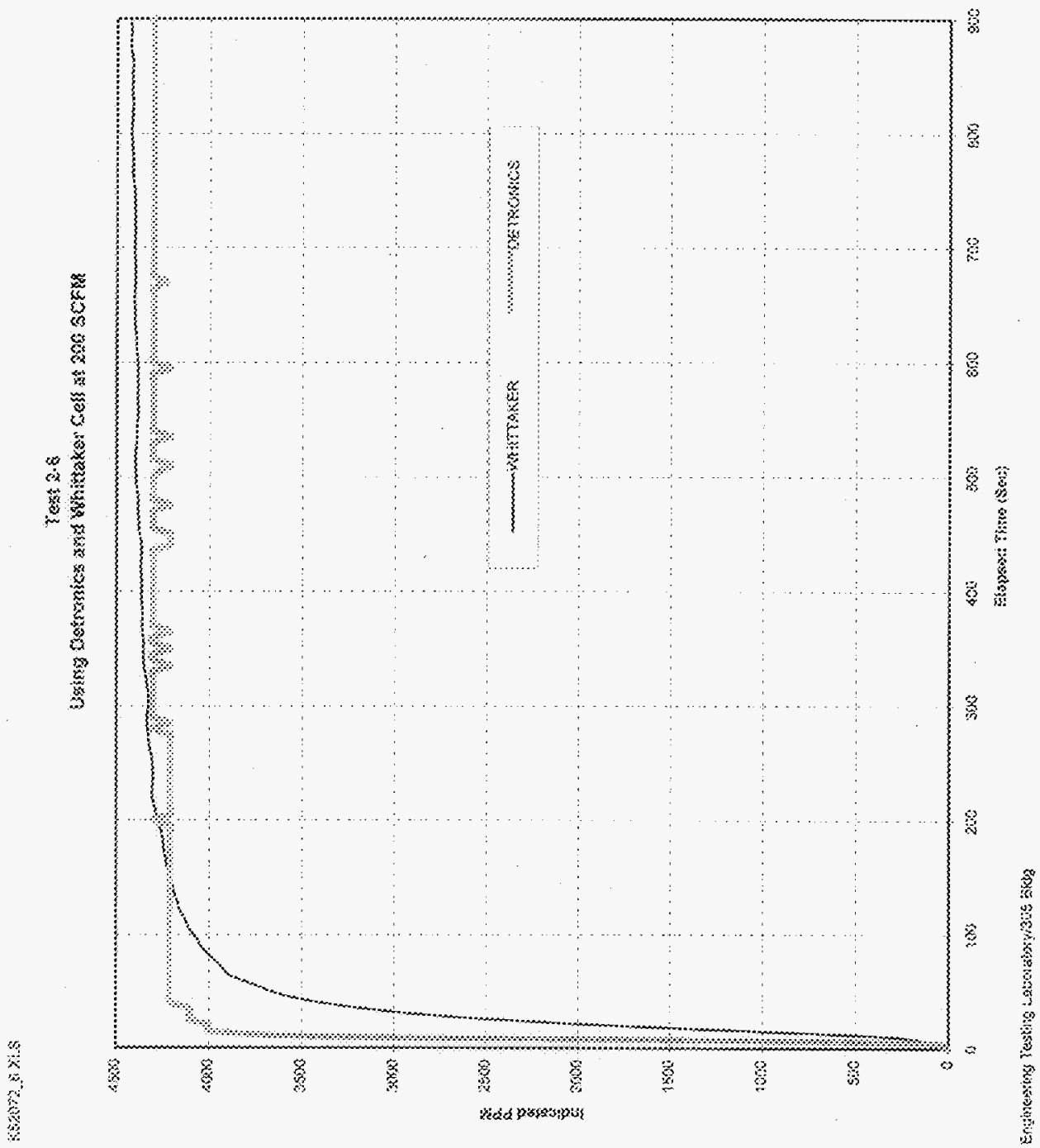




\section{FOPOW TOS BATA SHEET}

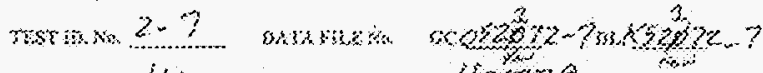

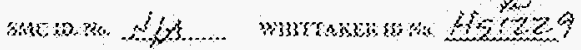

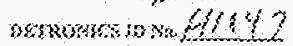

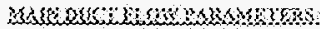

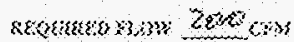

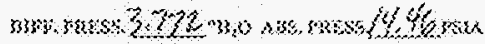

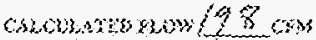

$32 \times 2,32.4$

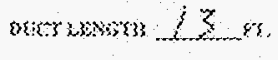

THoksory raks: sic:

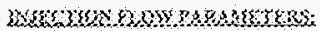

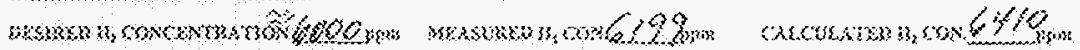

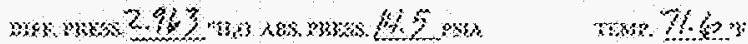

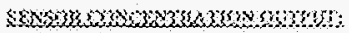

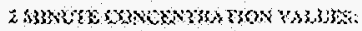

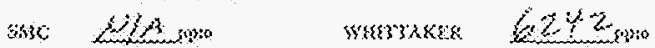

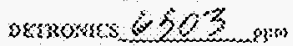

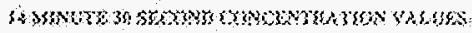

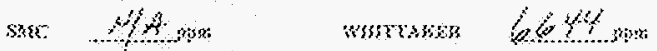

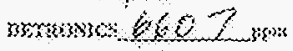

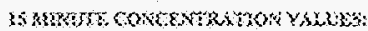

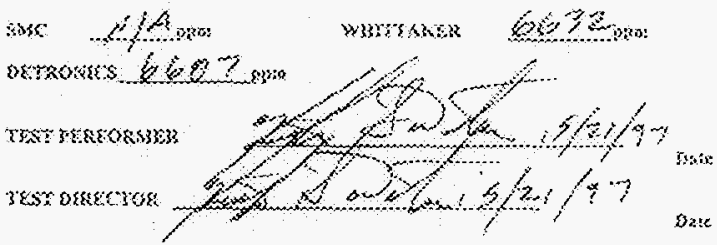


HW

$90 \mathrm{p}, 0$

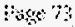

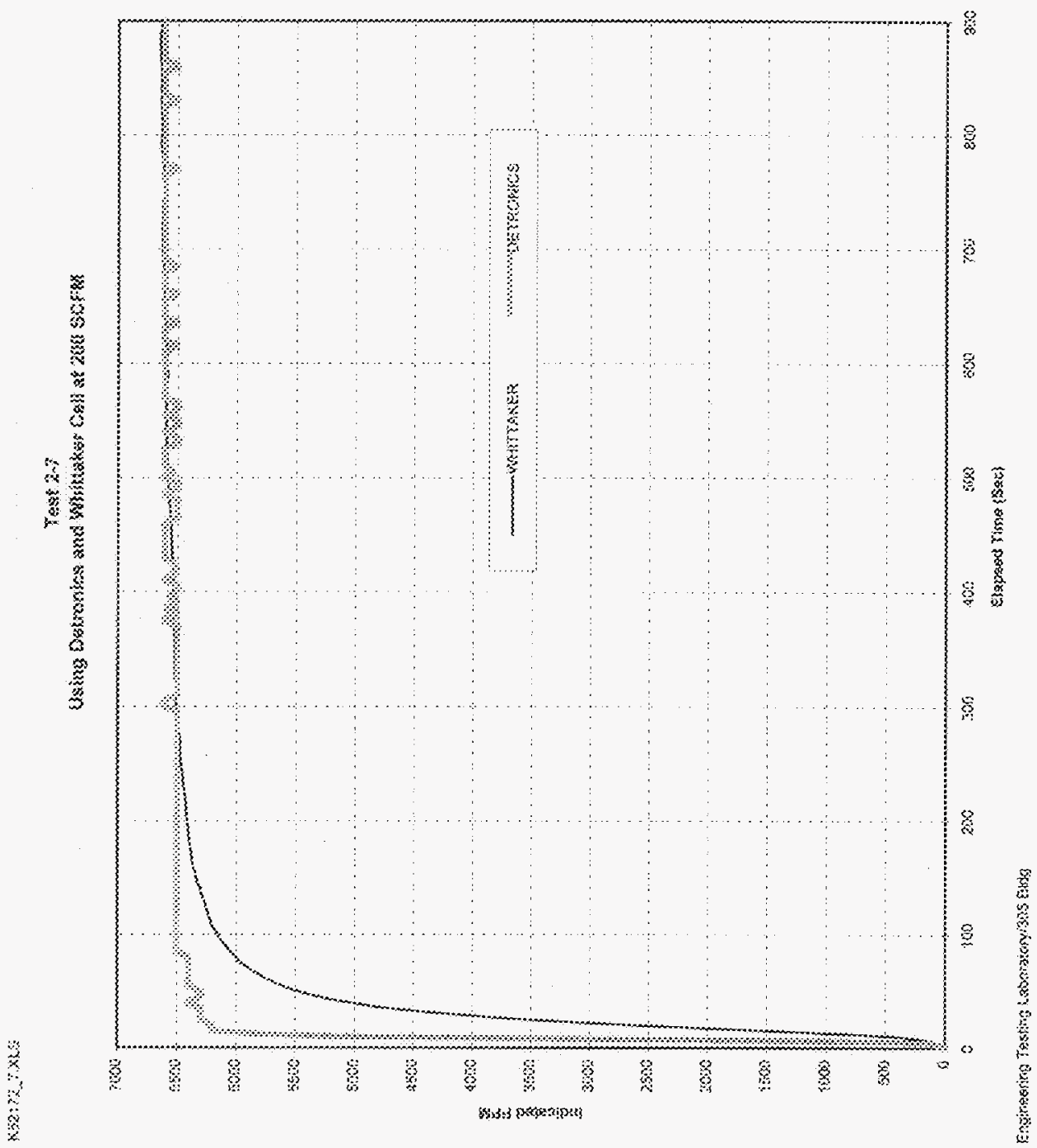




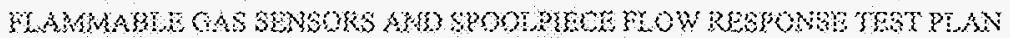
ANO EROCEDUK:

\section{PGULW WSTDAS SUEW}

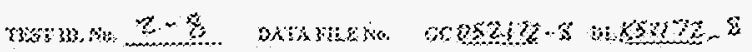

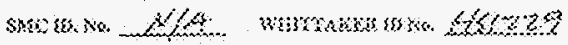

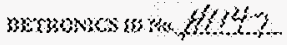

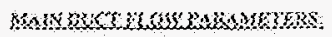

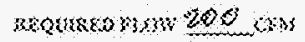

wownmstow $192 \mathrm{cms}$

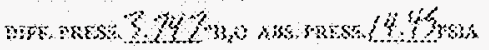

sess. $7 i$. $\%$

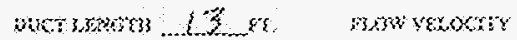
masc

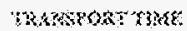
330

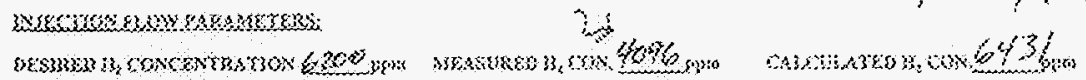

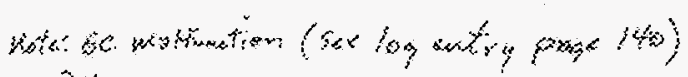

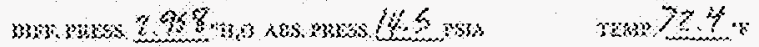

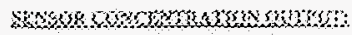

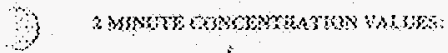

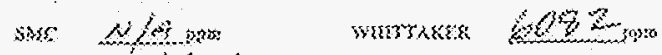

morsuses 0585

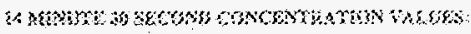

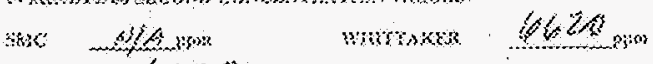

घerowes $340 \%$

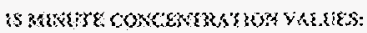

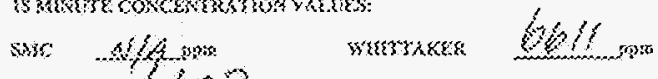

nemowes $\{602$

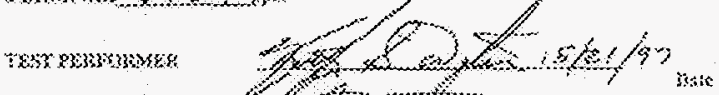

row 3mmeron 
काष

E.e. 6

foge 75

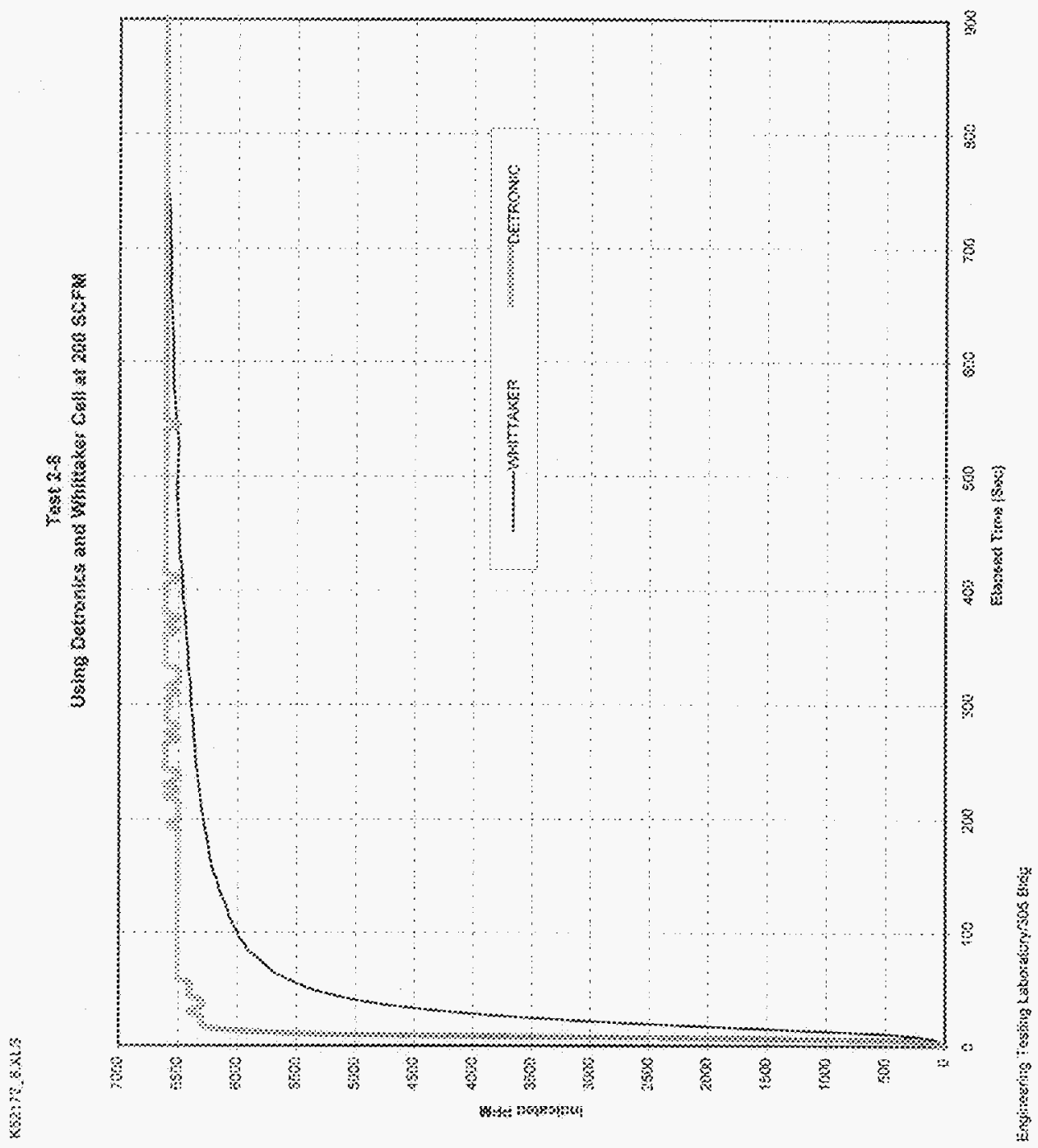




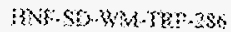

Serv. 0

Hog 78

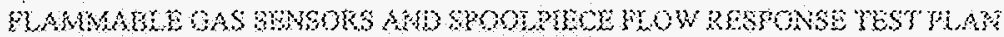
AND Kaoconoul

\section{GGMUN TES DATA SHERT}

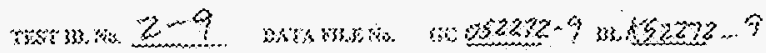

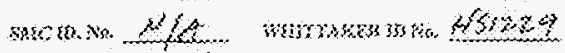

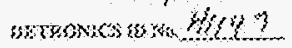

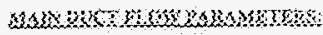

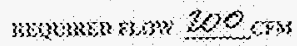

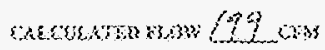

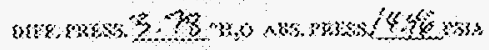

wat $7 \%$

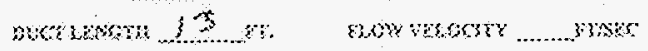

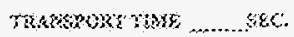

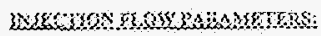

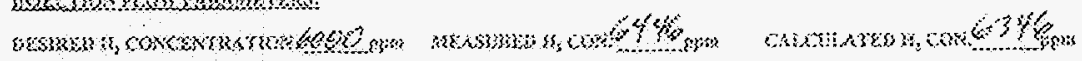

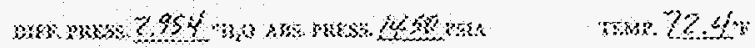

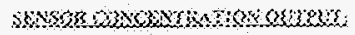

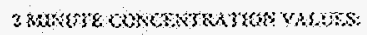

she $\alpha / 2$ wish

vasemes is 503

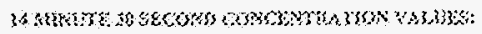

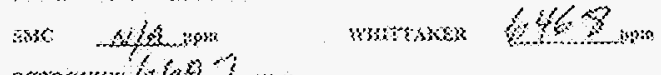

ansoms

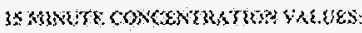

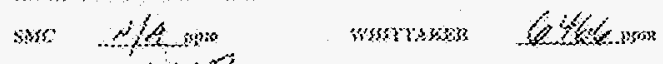

werowas $<6427$

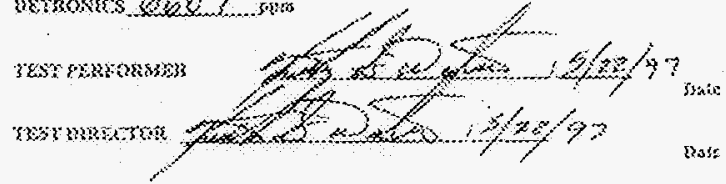


:Avy. \$5-342-7ap-?86

iter. 0

Prece $7 \%$

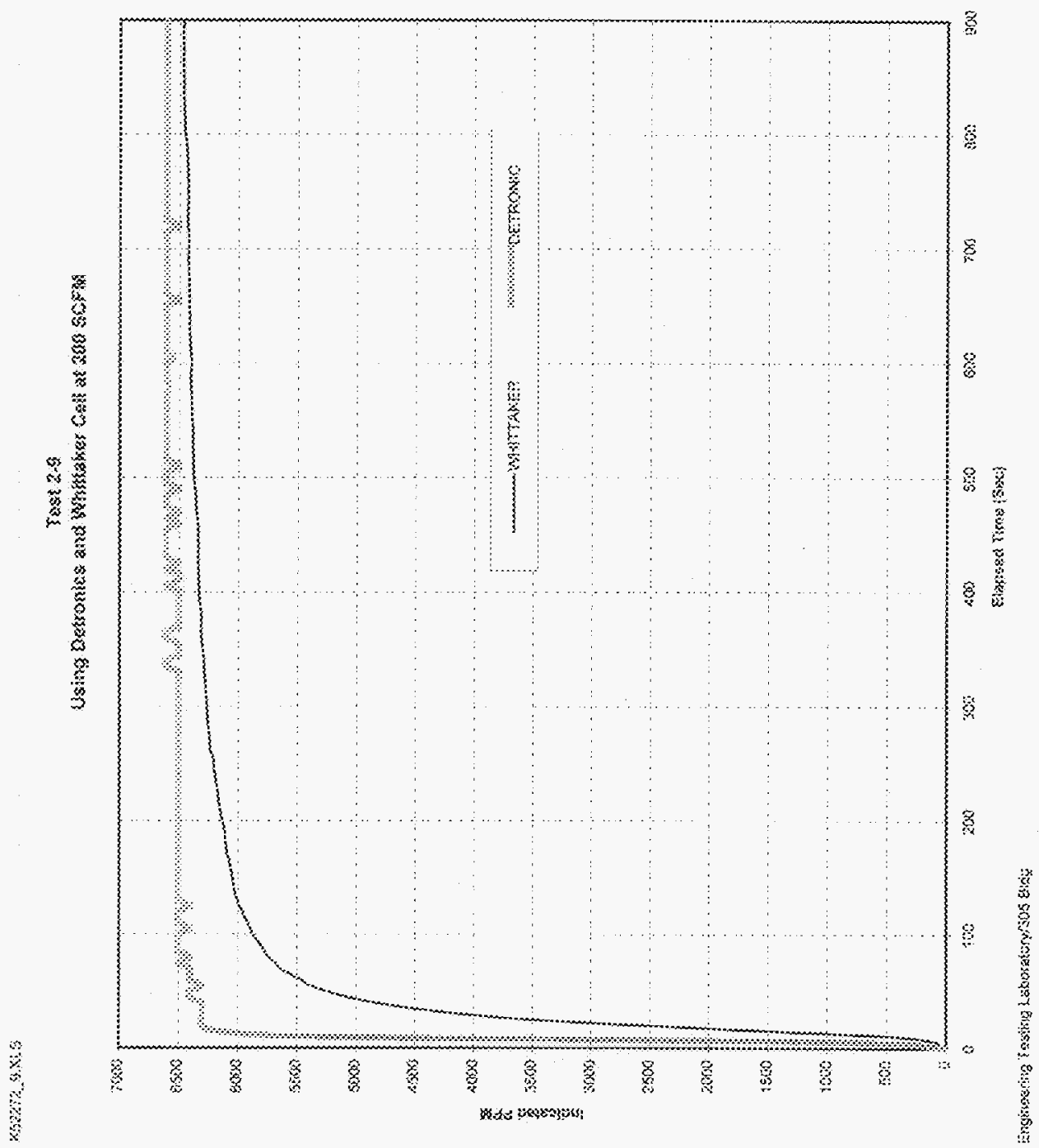



AMO PEOCWOUS:

\section{EOT WOW USET DATA BHEDT}

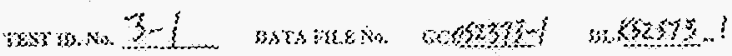

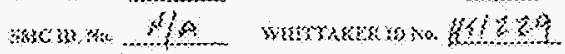

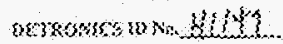

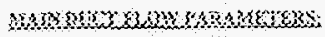

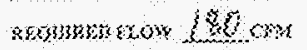

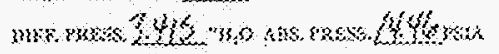

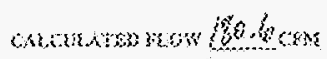

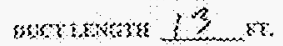

$\sin 2640$

mลง se.

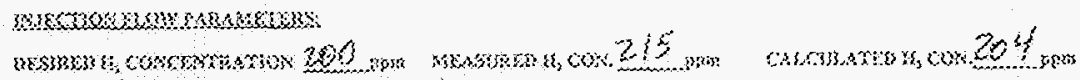

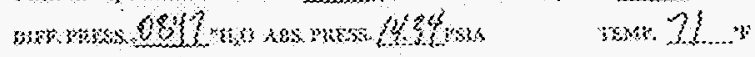

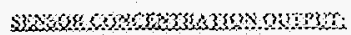

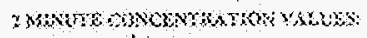

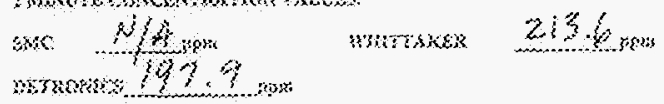

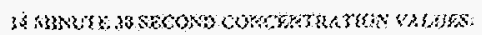

sms $\mathrm{x} / \mathrm{f}$ spos

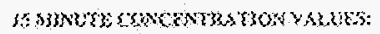

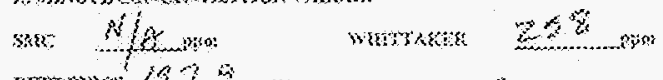

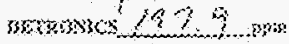

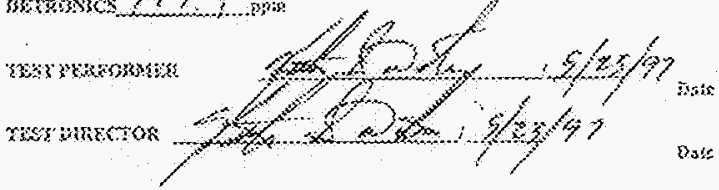




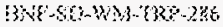

veres 6

Quse 75

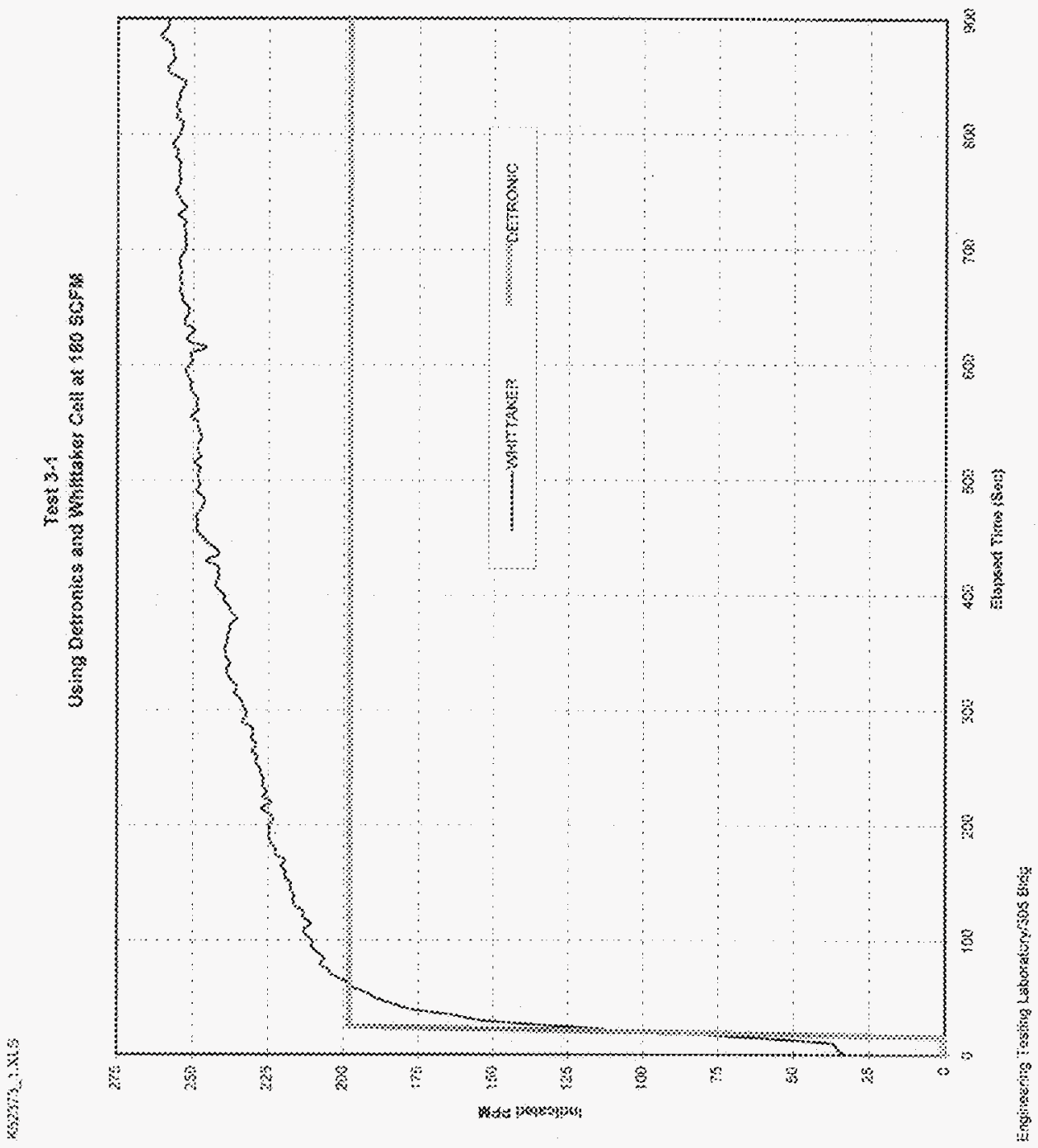




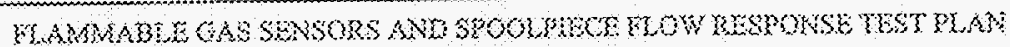

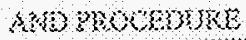

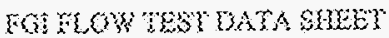

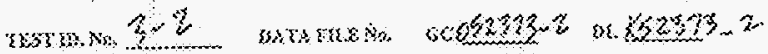

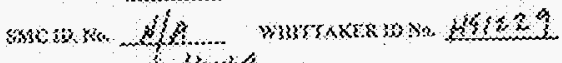

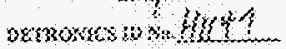

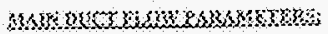

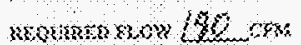

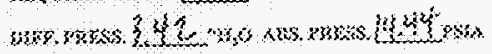

$\operatorname{cosinaxesmosm~} 180$

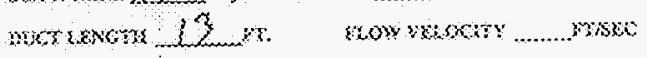

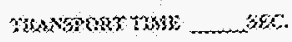

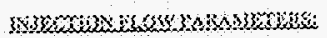

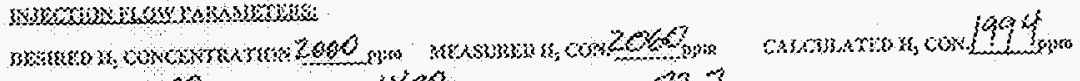

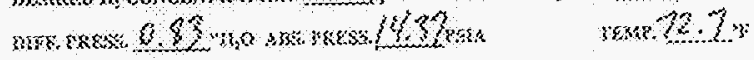

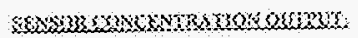

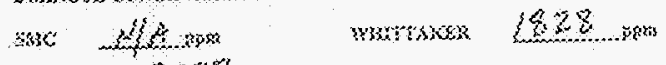

weskonses $200 \%$

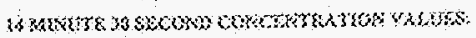

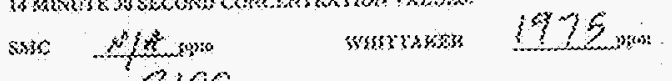

promoras $\frac{2}{3} \leq 2$

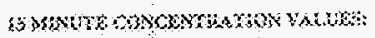

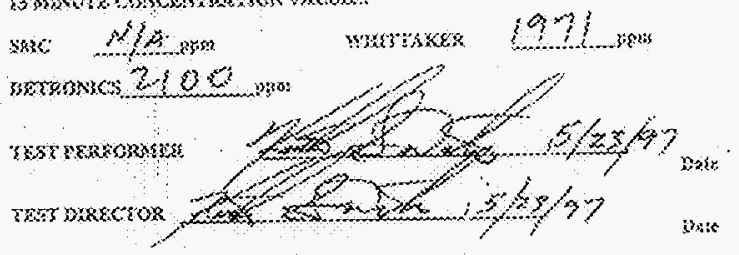




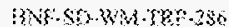

\$ex. 0

[so : : :

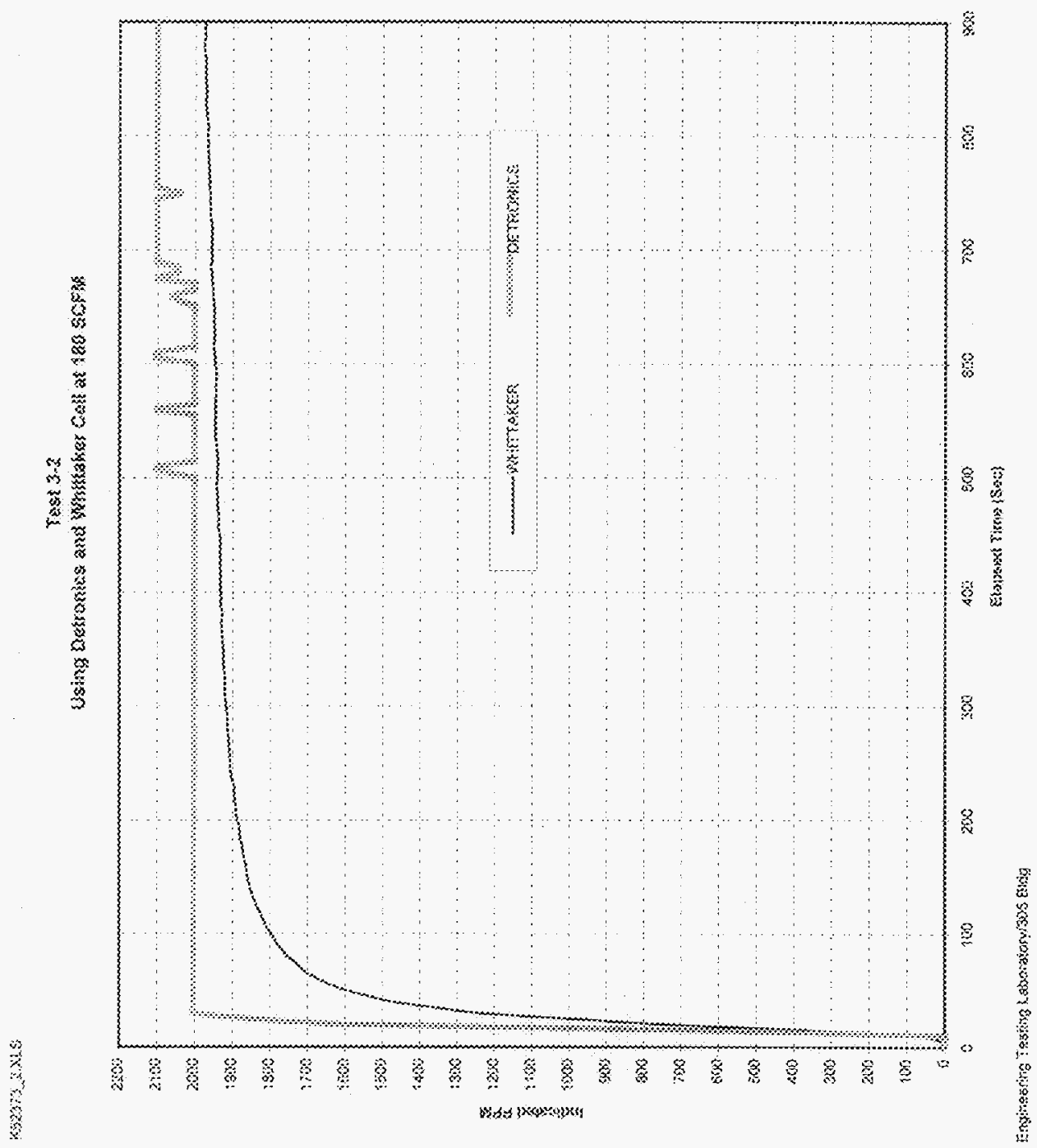




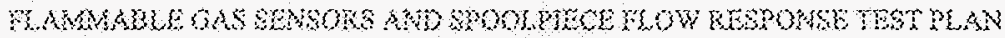
ANo ranomouns

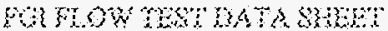

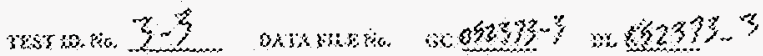

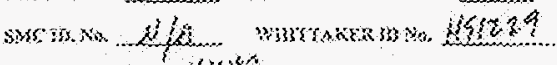

manowes the 4 ily

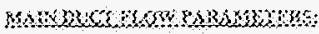

nerwsen whw $/ 80 \mathrm{sm}$

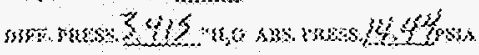

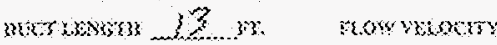
sis:

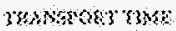

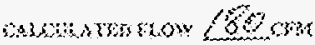

swis. $30.8 \%$ masc

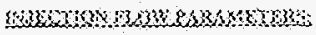

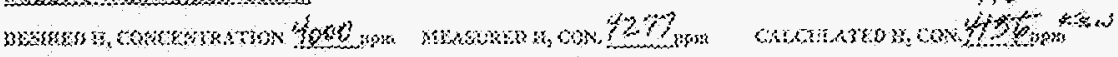

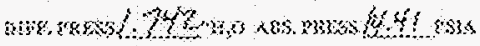

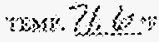

3600R co

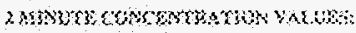

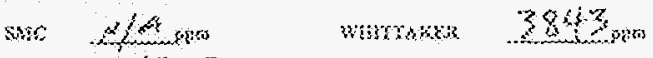

venowes 130

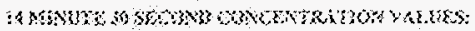

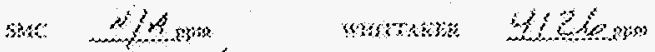

maroxise 24204

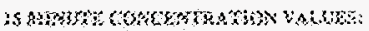

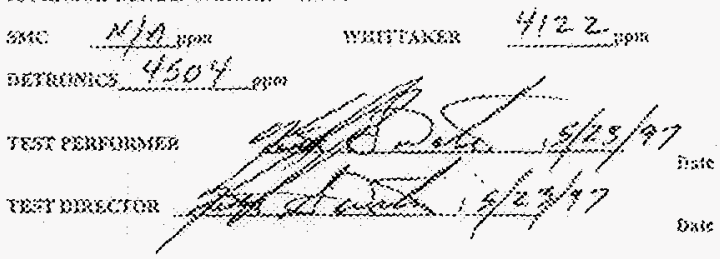




$$
\begin{aligned}
& \text { 45E-S.WM-\{\}?-2R } \\
& \text { gor. } \\
& \text { fes } 83
\end{aligned}
$$

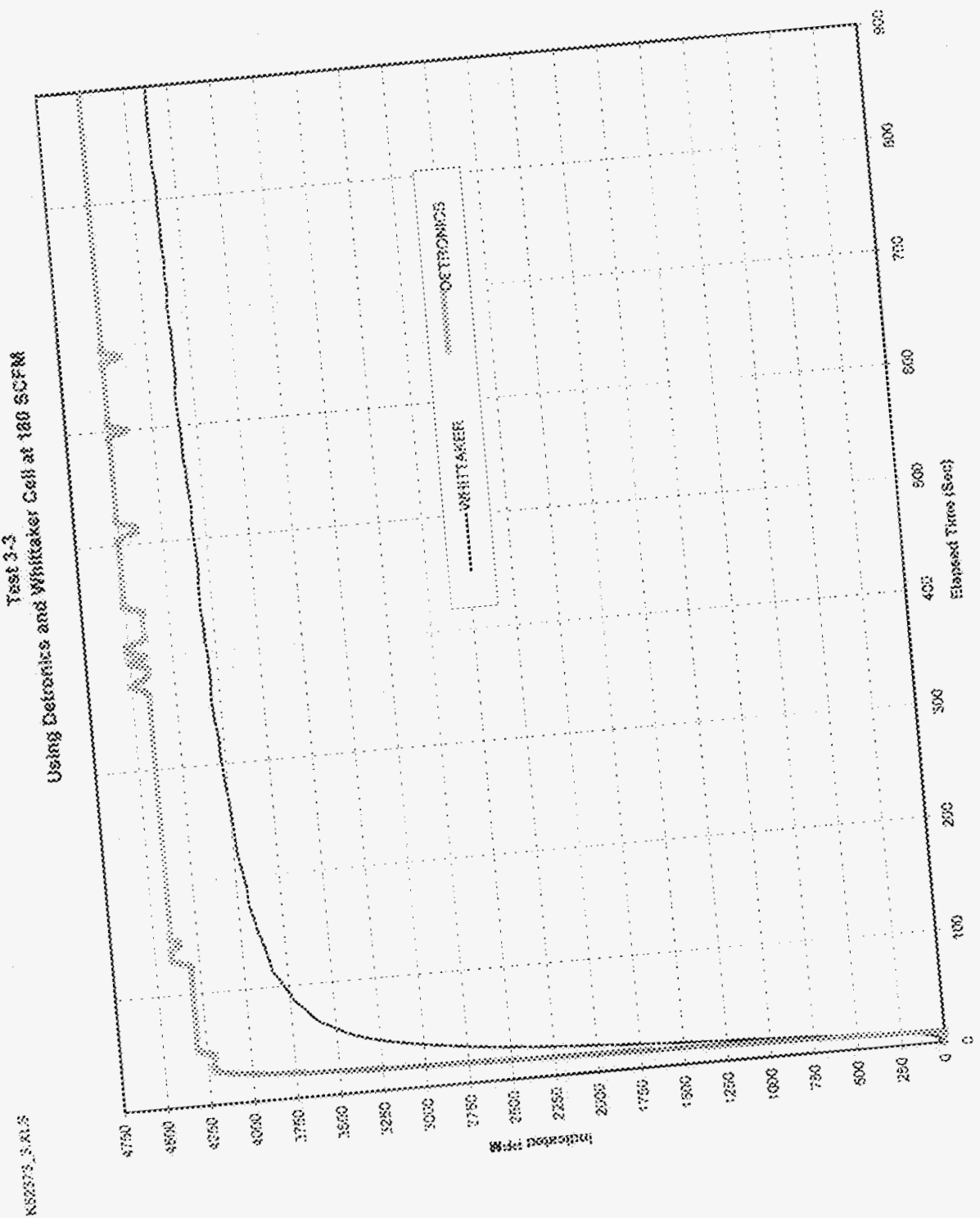




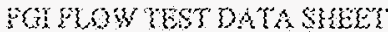

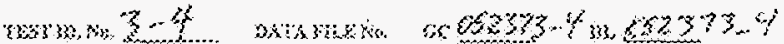

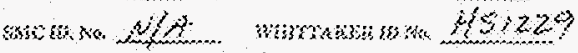

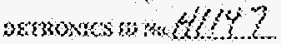

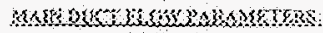

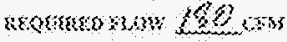

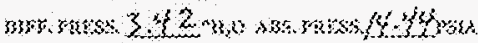

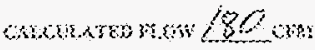

$\operatorname{man} 3: 3.3$

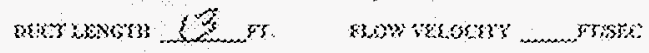

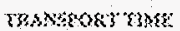
$\$$

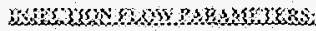

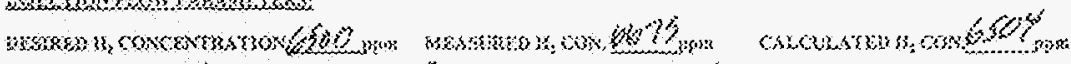

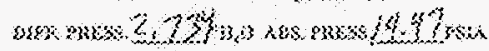
rom: 25

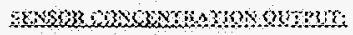

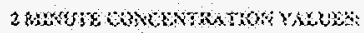

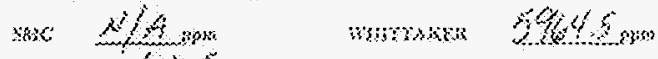
DesRowisc $64 \mathrm{~g}$

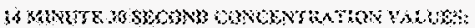

whe $4 / 4$ mon

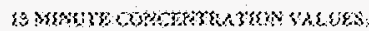

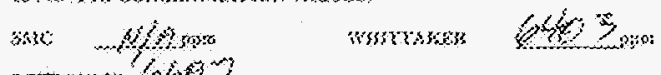

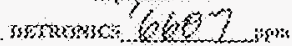

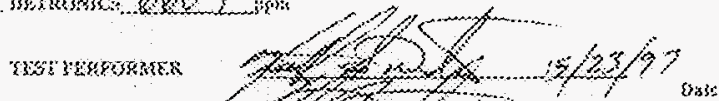

Merr whesess 


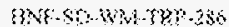

ser. 0

Exye :15

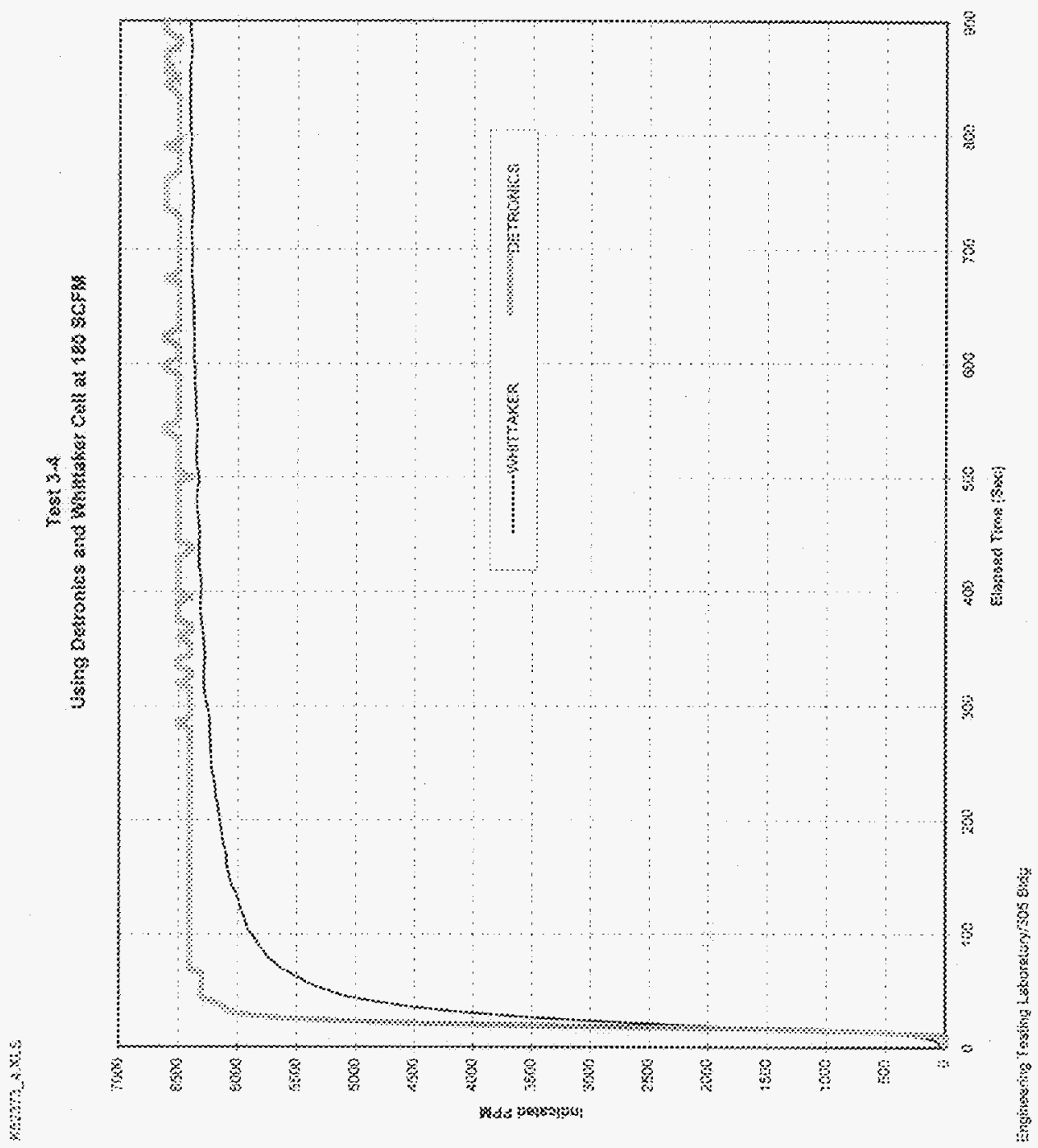




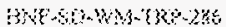

Q.?. 0

Pxgo 96

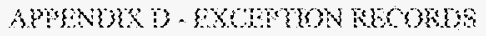




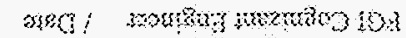

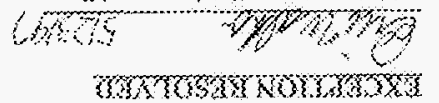

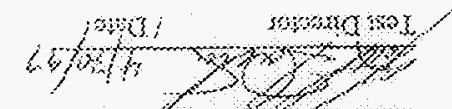

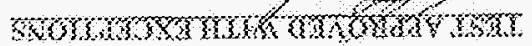

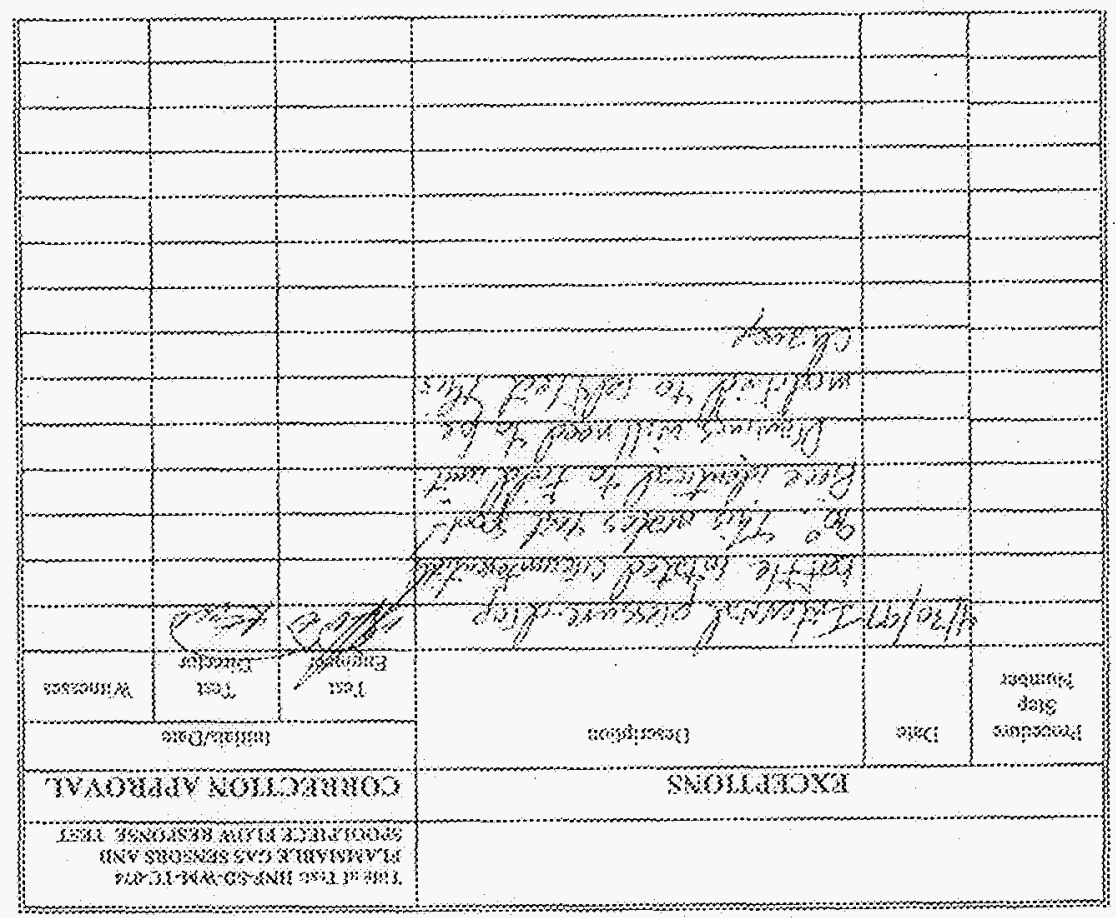

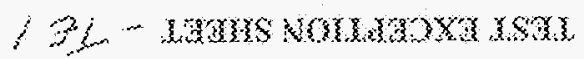

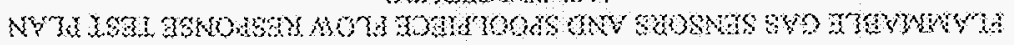

$2013 x_{i}$

i) $20, \mathrm{i}$

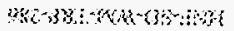




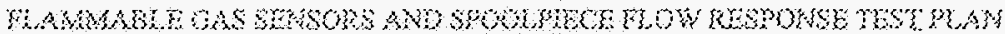
ADPQOWWWE

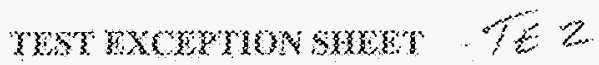

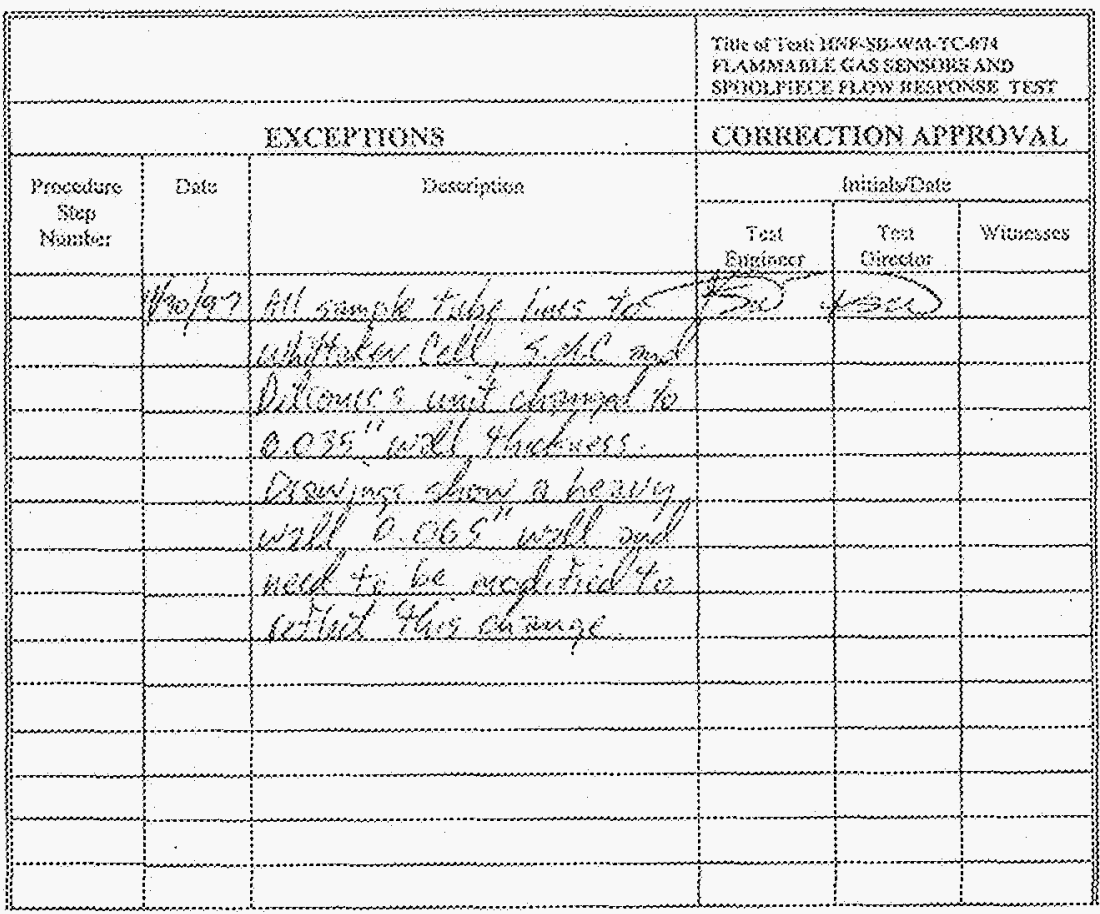

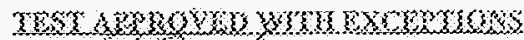

\section{Sest Obonos}

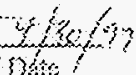

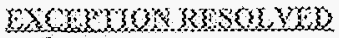

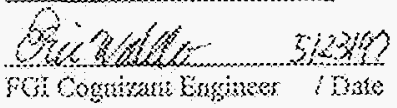




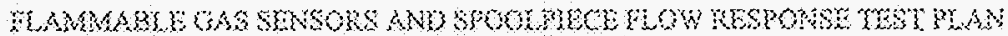

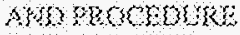

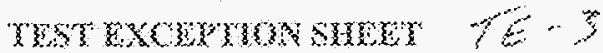

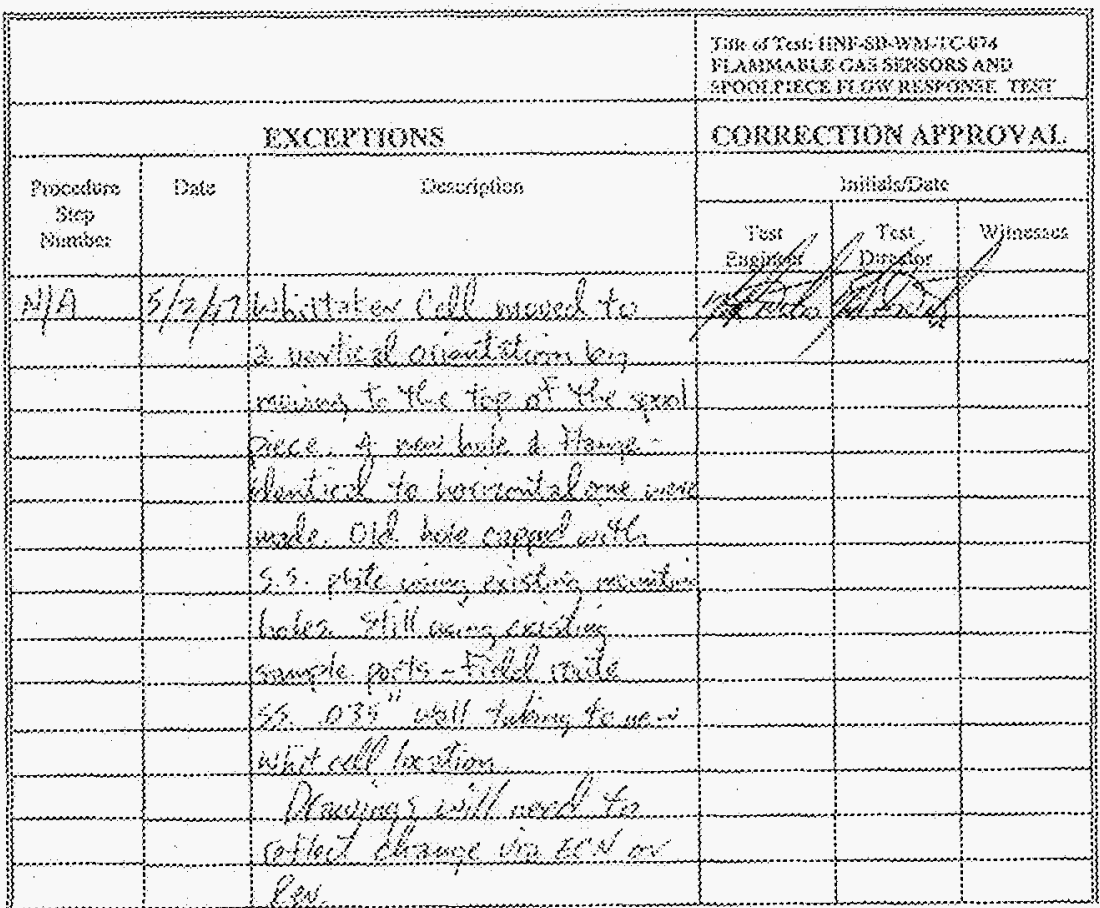

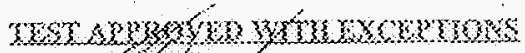

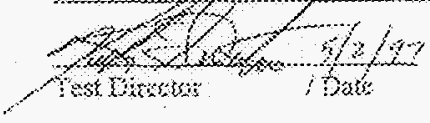

EXYMWUAMESYSO

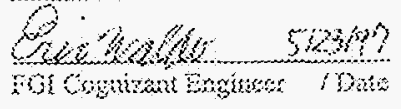




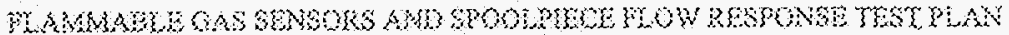

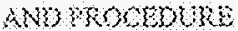

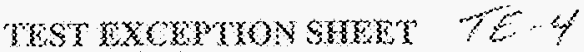

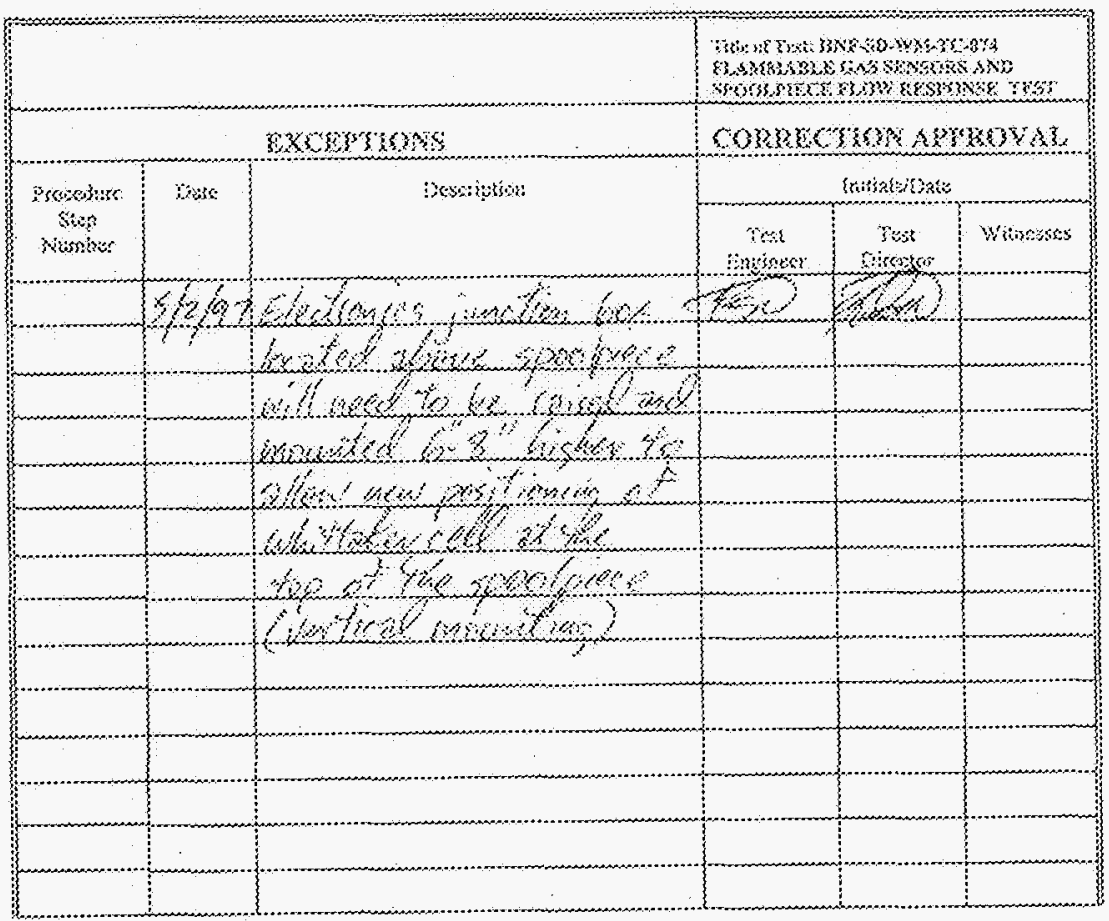

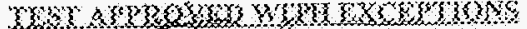

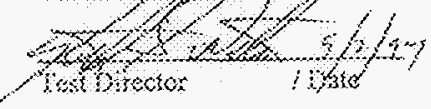

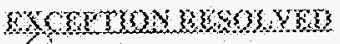

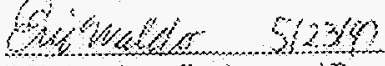

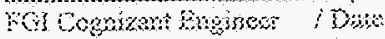




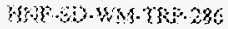

$33 \times 0$

? 3 ?

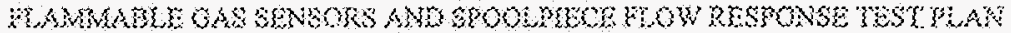

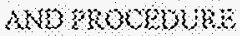

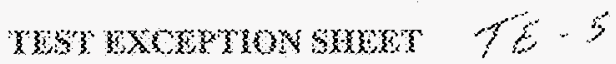

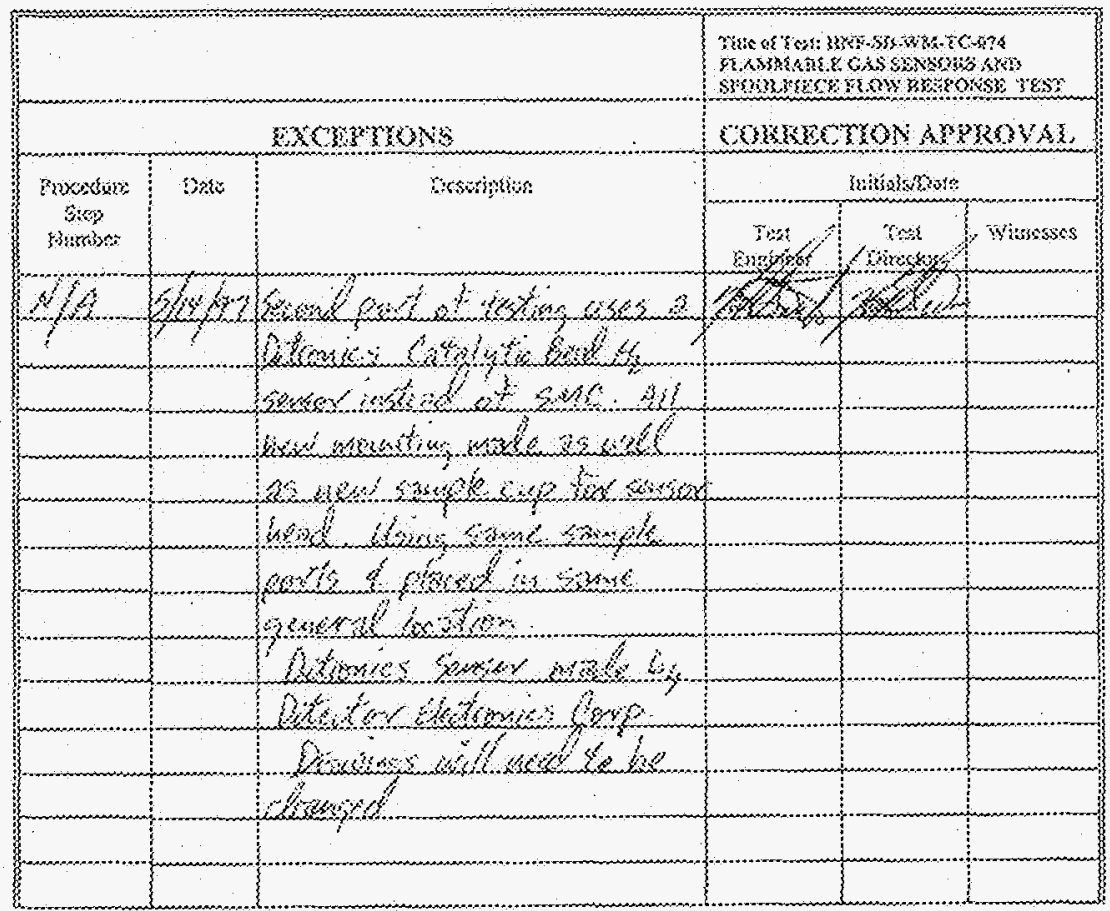

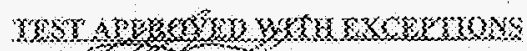

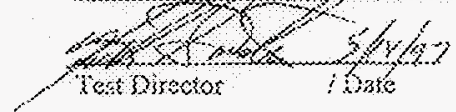

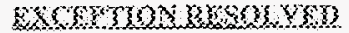

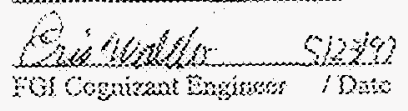




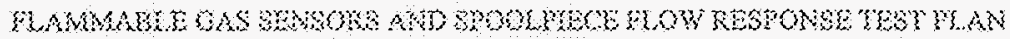
sNo pरoroutike

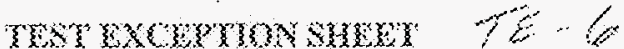

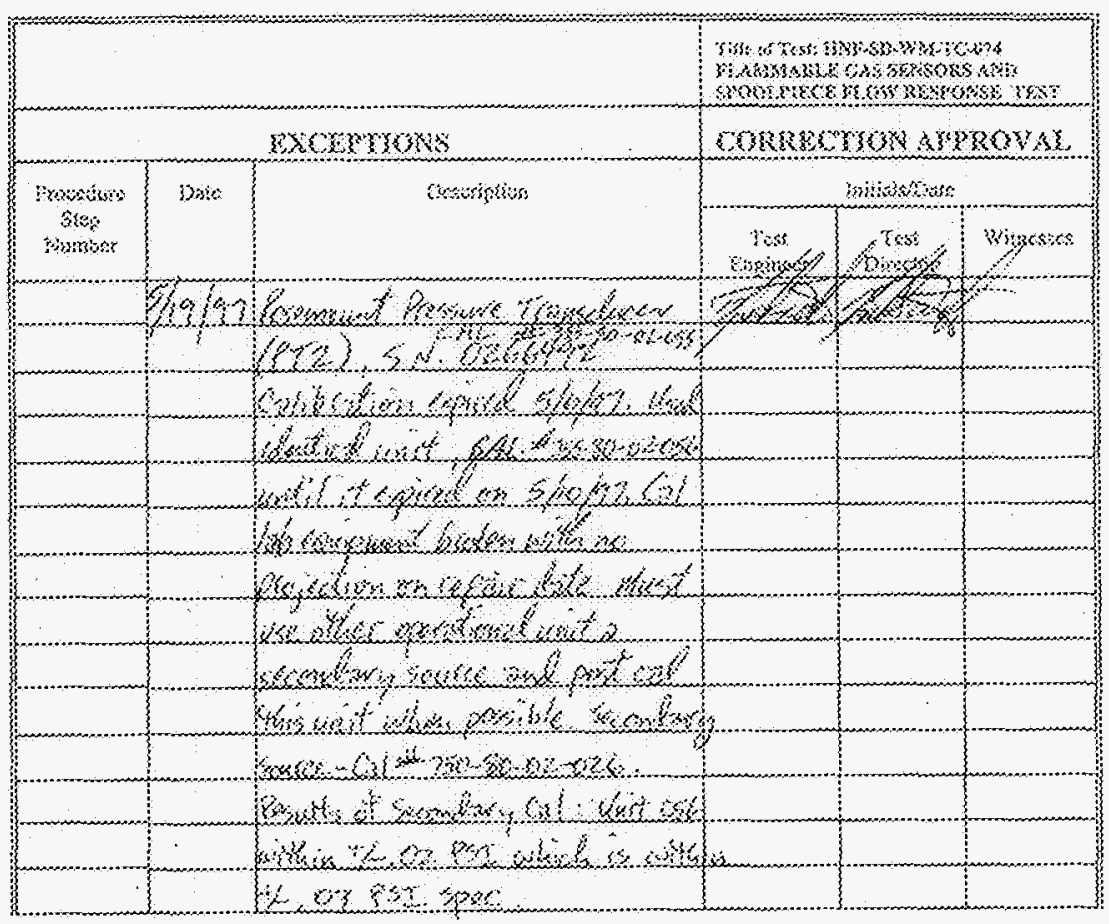

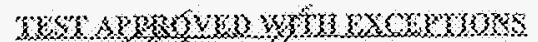

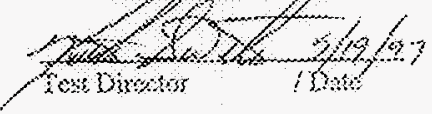

Exomson

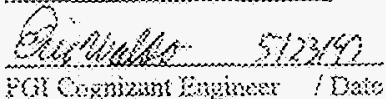




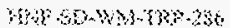
ser. 0

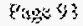

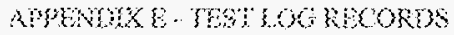




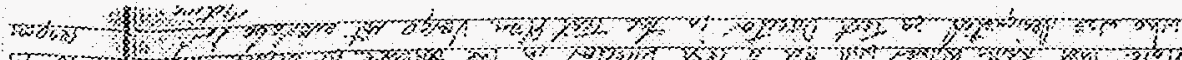

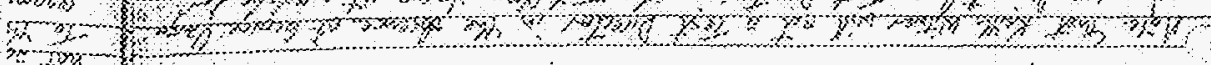

trom Hom

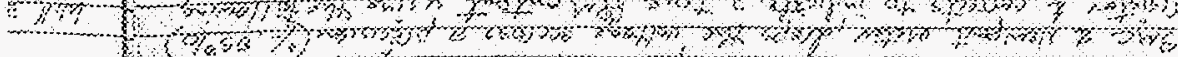

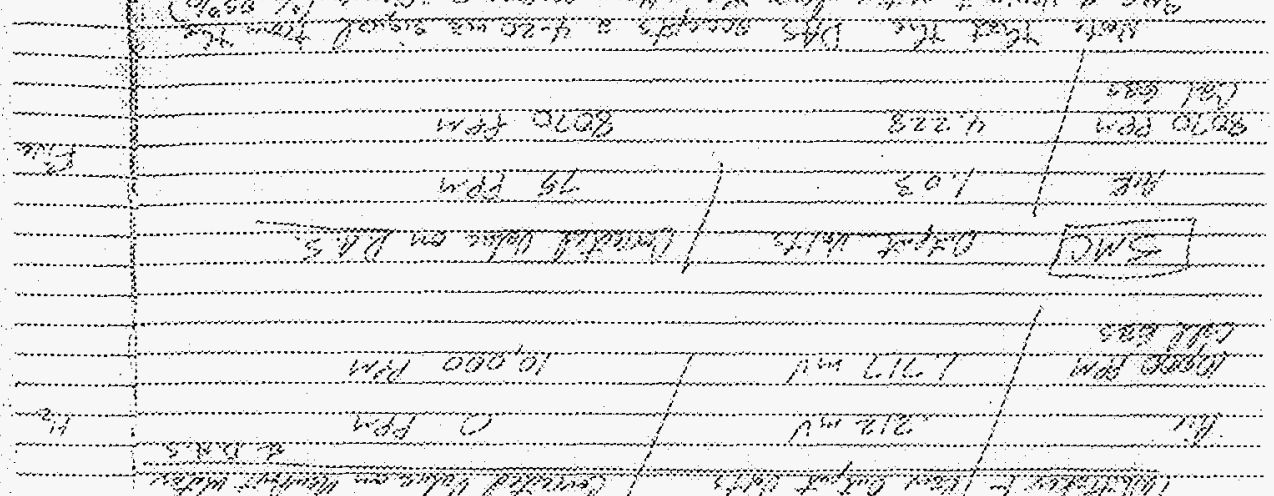

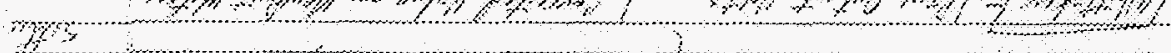
Ho $\quad$ w mo

in $\quad$, W
a

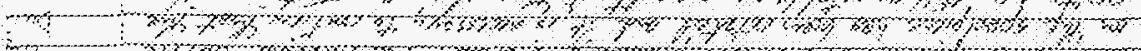

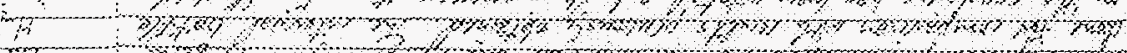

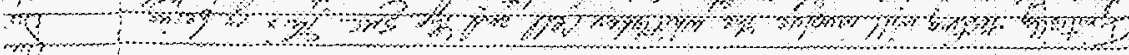
2)

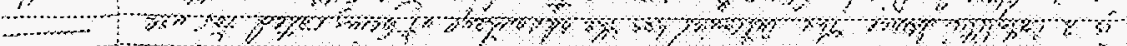

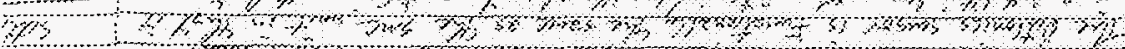
अ

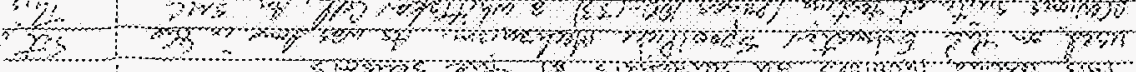

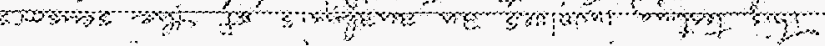
$\cos / \infty$

53

6) AaX:

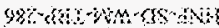




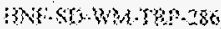

Kes.

pogs

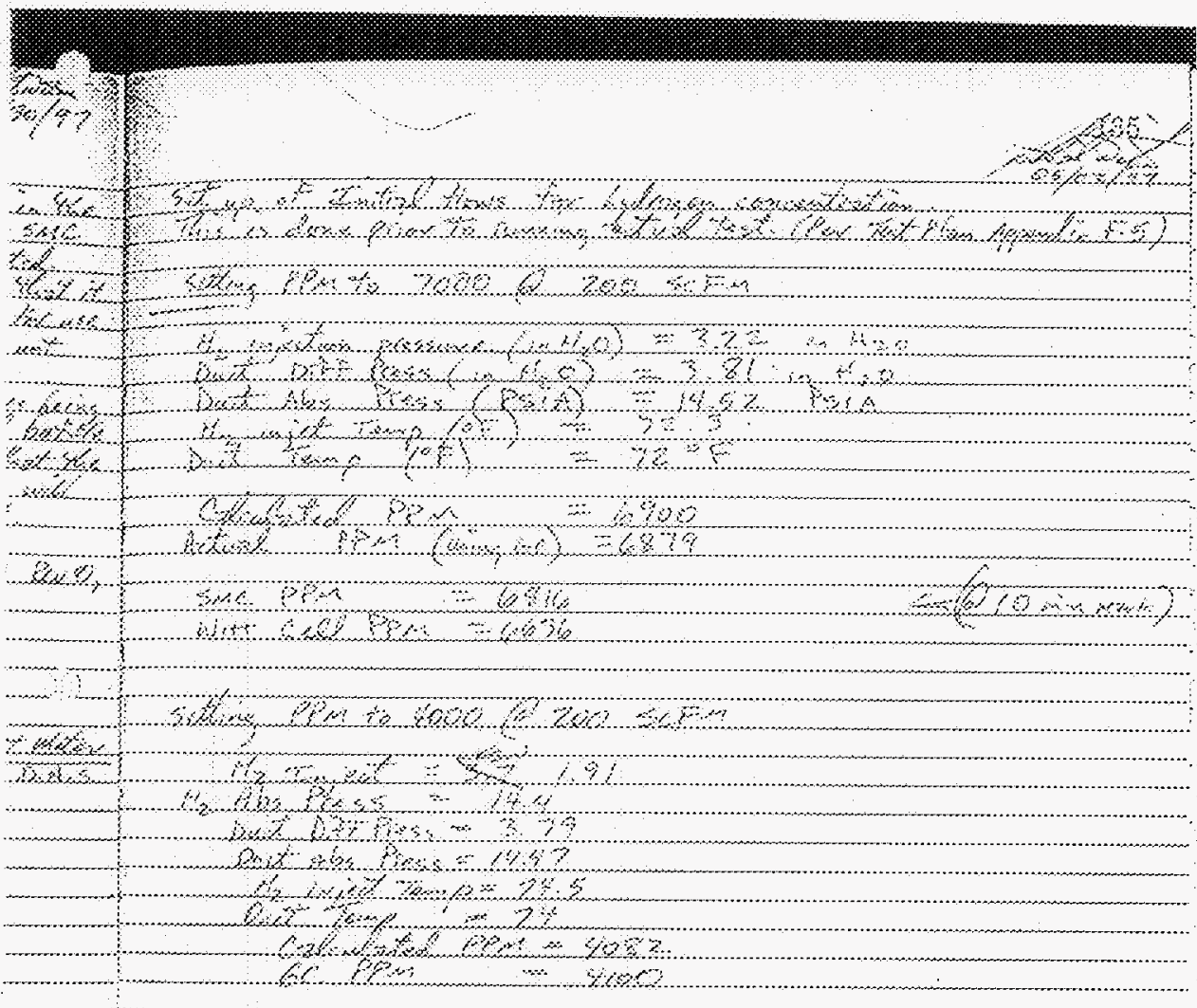

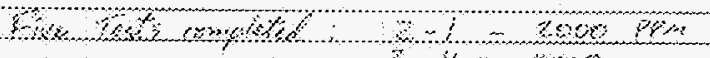

$2-y-1200$
$2-3-200$
$2-8-200$
$20-2000$

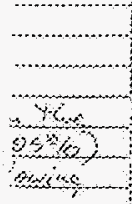
(n)

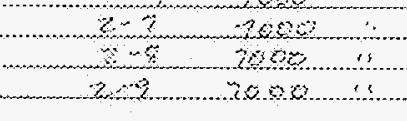

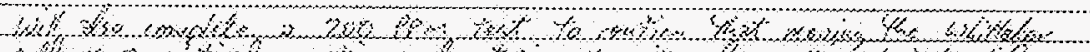

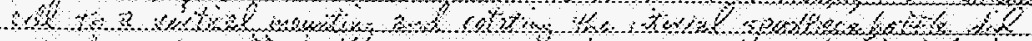

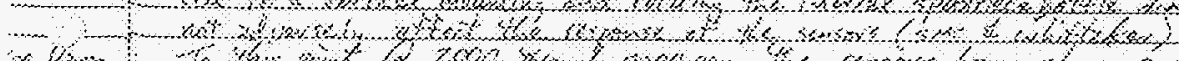

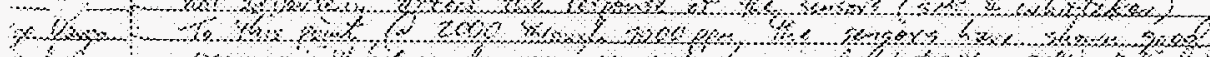

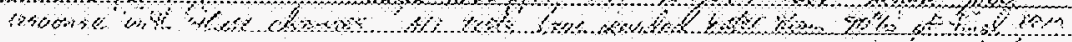




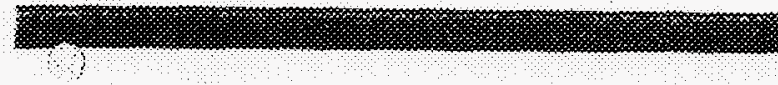

336

-.

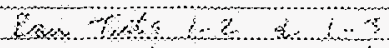

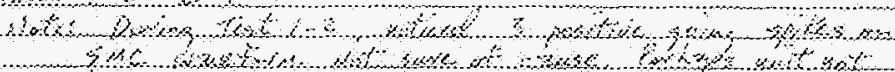

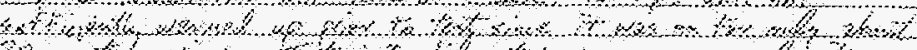

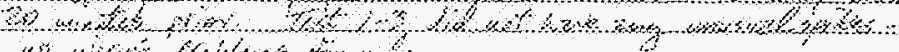

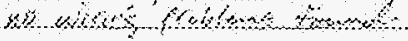

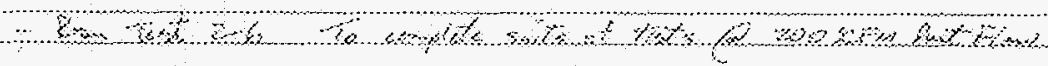

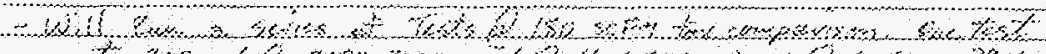

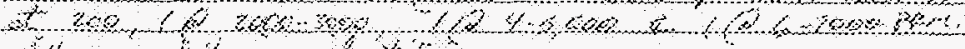

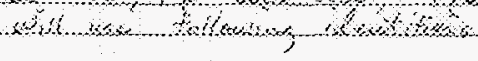

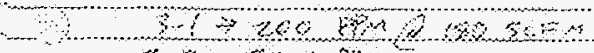

$x+200$ of

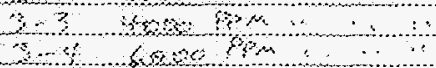

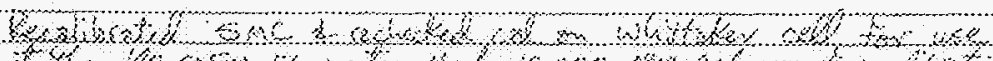

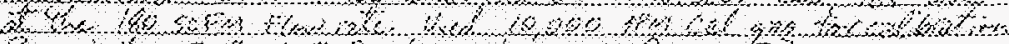

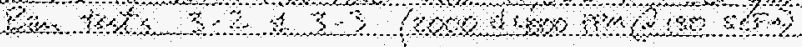

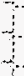

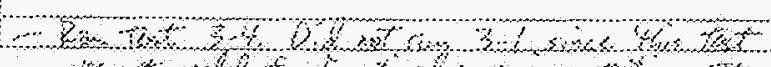

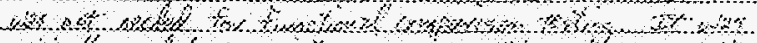

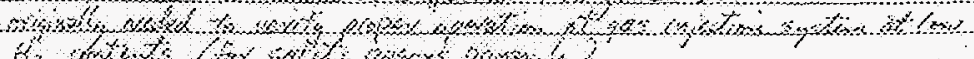

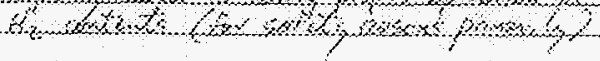

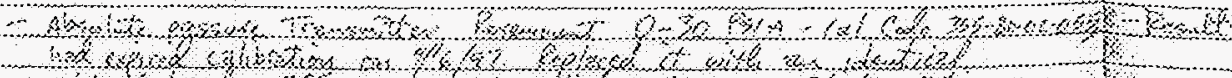

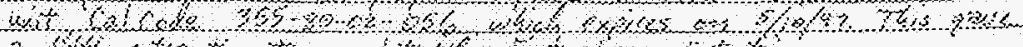

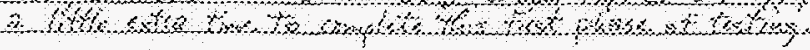




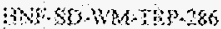

ker.

toge

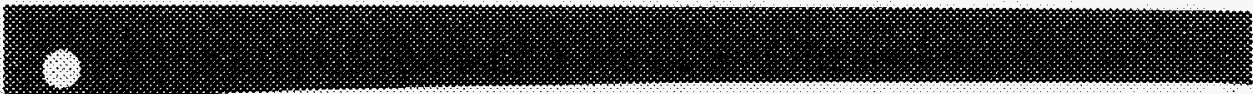

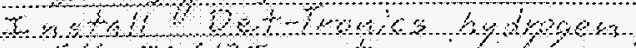

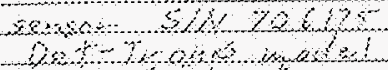

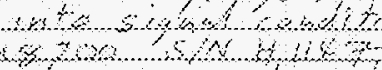

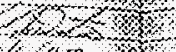

B.t?

\section{..................}

$+\ldots$

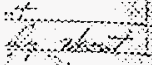

NAs 1

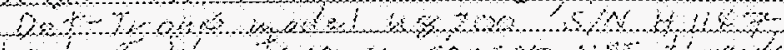

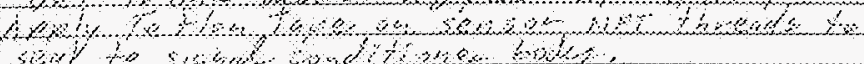

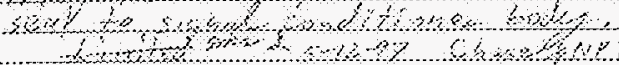

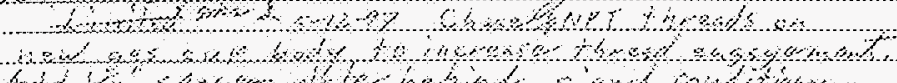
Kal

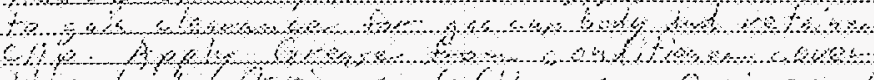
atowit. H.

ats.

is.

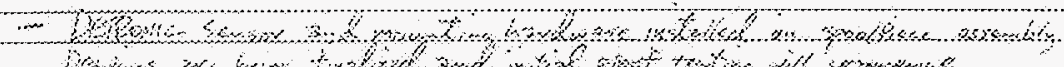

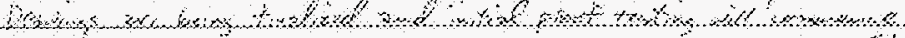

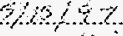

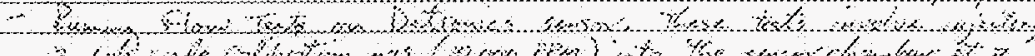

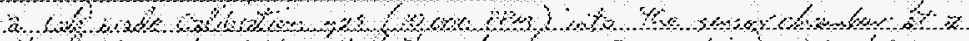

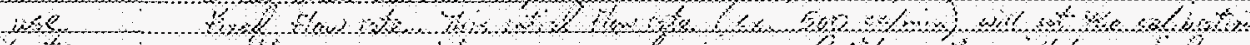
beresca

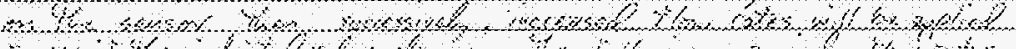

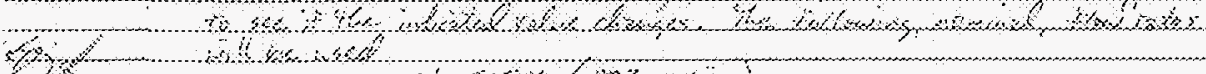

tats $? \sqrt{2}$

ros:

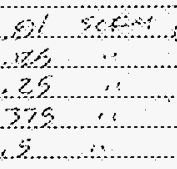

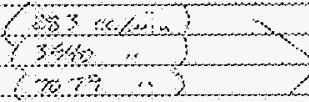

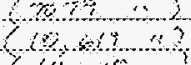
$(6,103,1)$

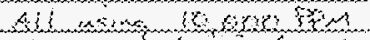
sitz-

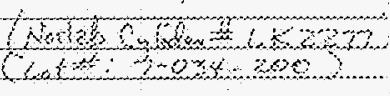

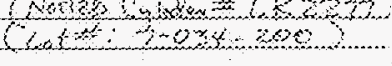

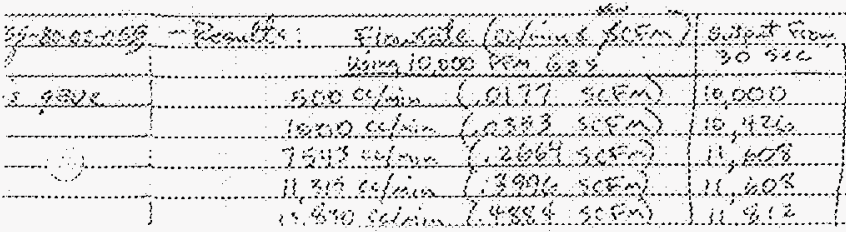

\section{$+\infty$}




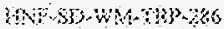

Kev. is

boses

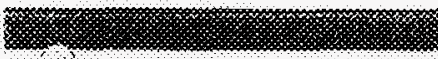

3.38

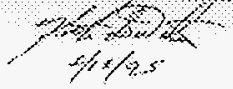

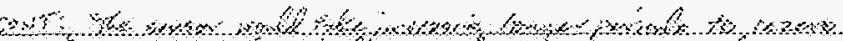

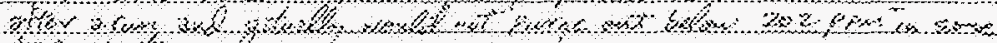

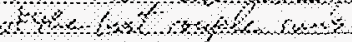

W

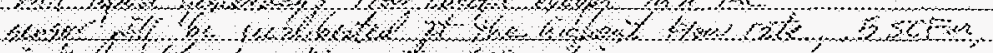

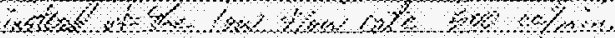

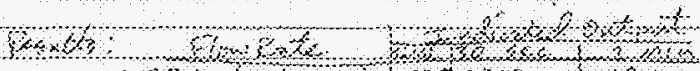

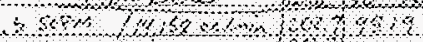

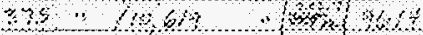

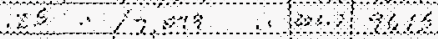

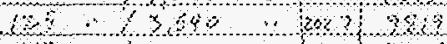

Qxy *

0130,500

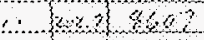

$\therefore$ 2at 2009

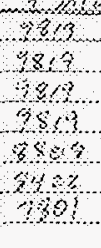

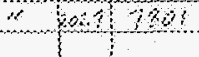

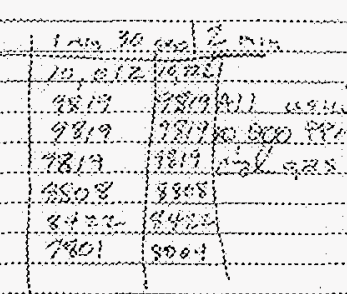

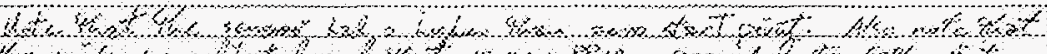

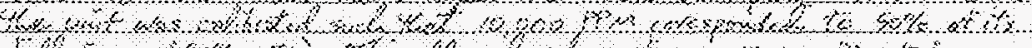

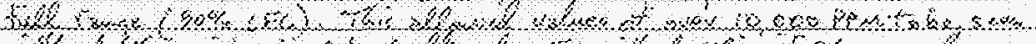

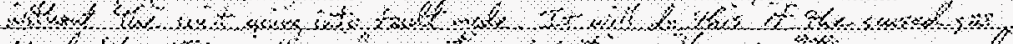

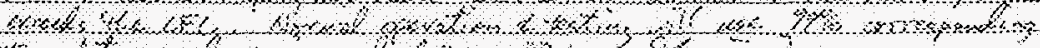

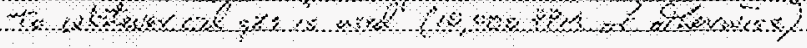

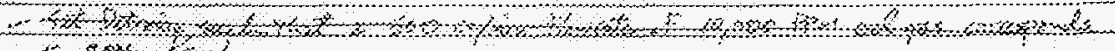
H. $8 x, 20,2$

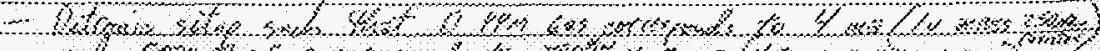

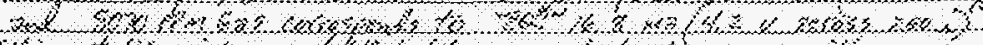

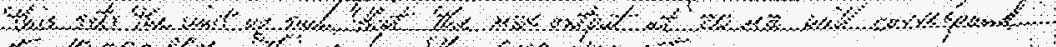

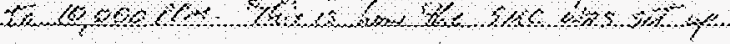

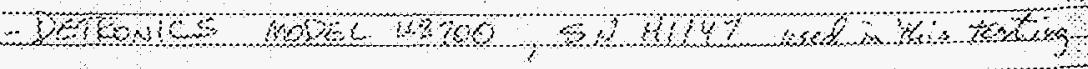

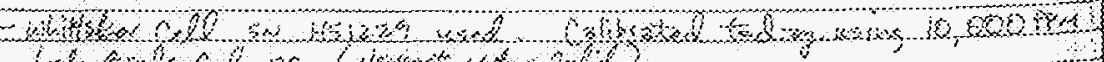

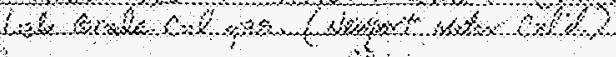

$$
\text { en }=0,08 \mathrm{~m}
$$

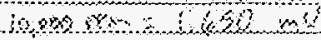




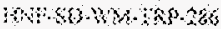

i)

Hase th

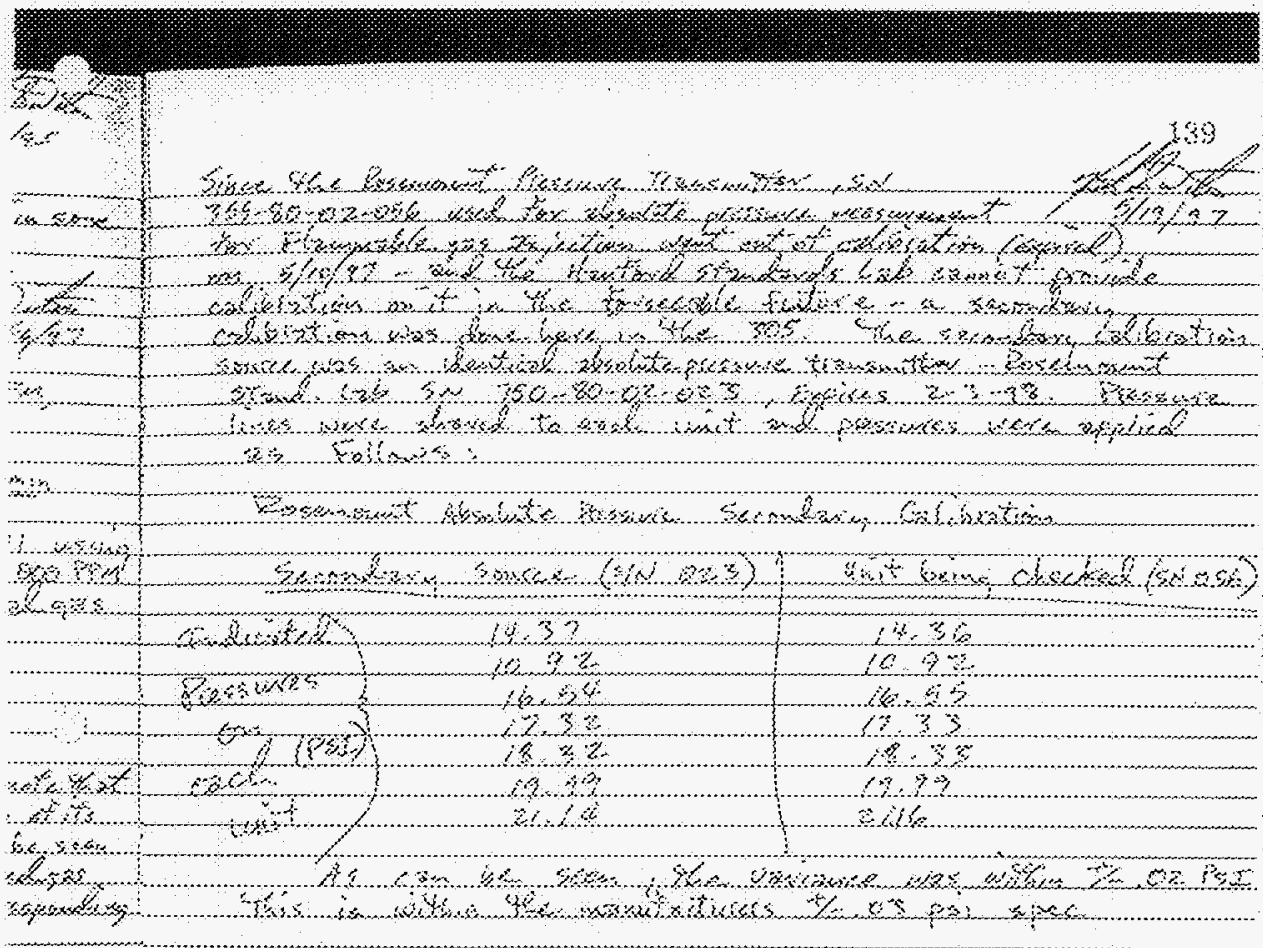

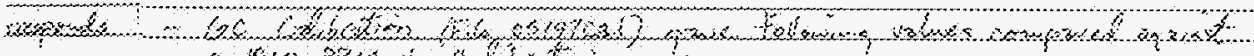

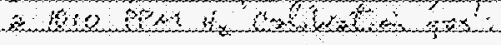

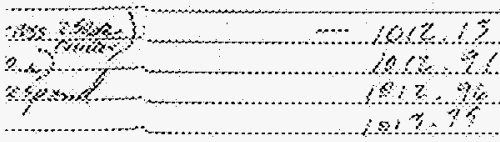

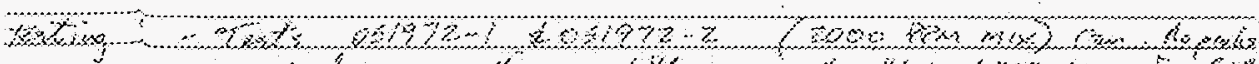
OS1M

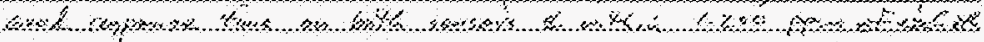
wow Po

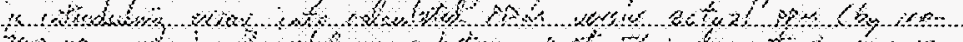

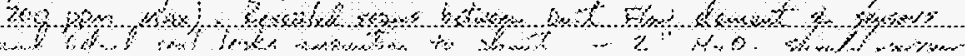




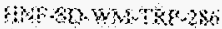

As: 0

(x) $:(B)$

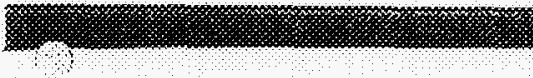

$1 / 0$

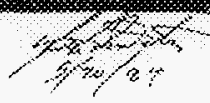

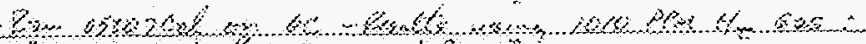

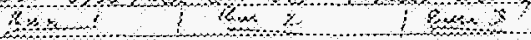

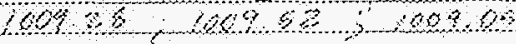

Pesibs

atis: fsol

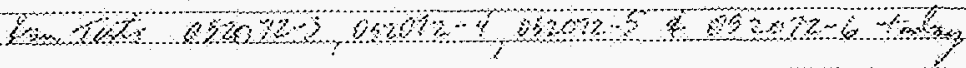

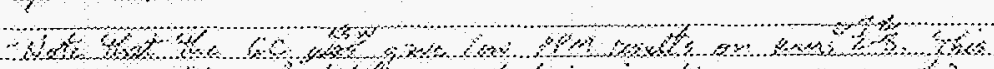

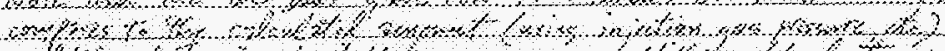

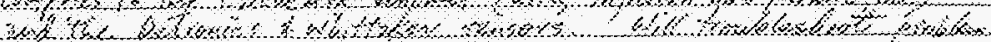

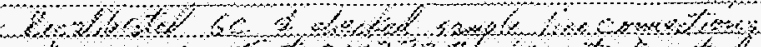

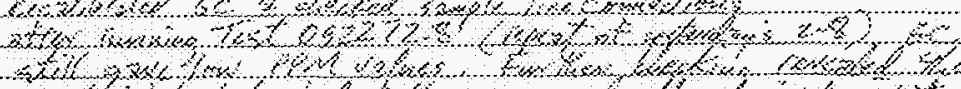

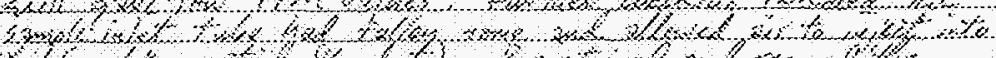

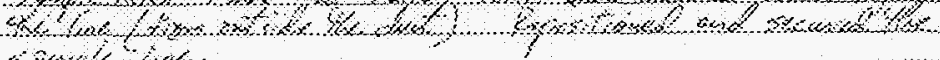

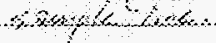

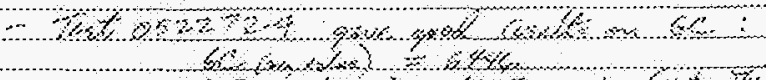

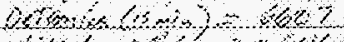

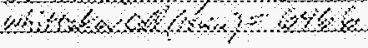

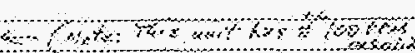

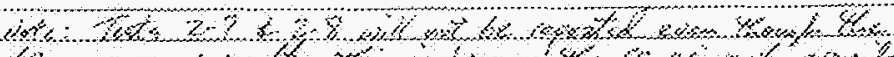

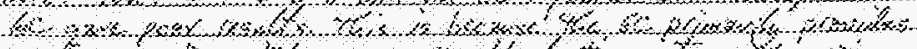

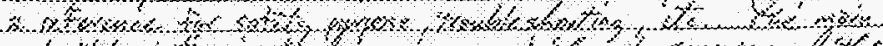

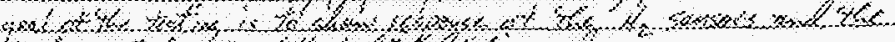

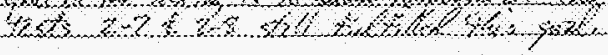

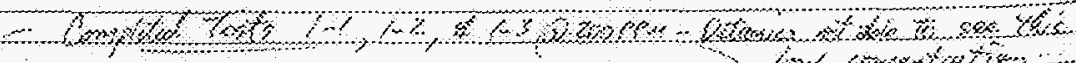

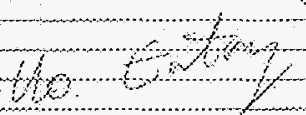

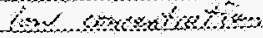




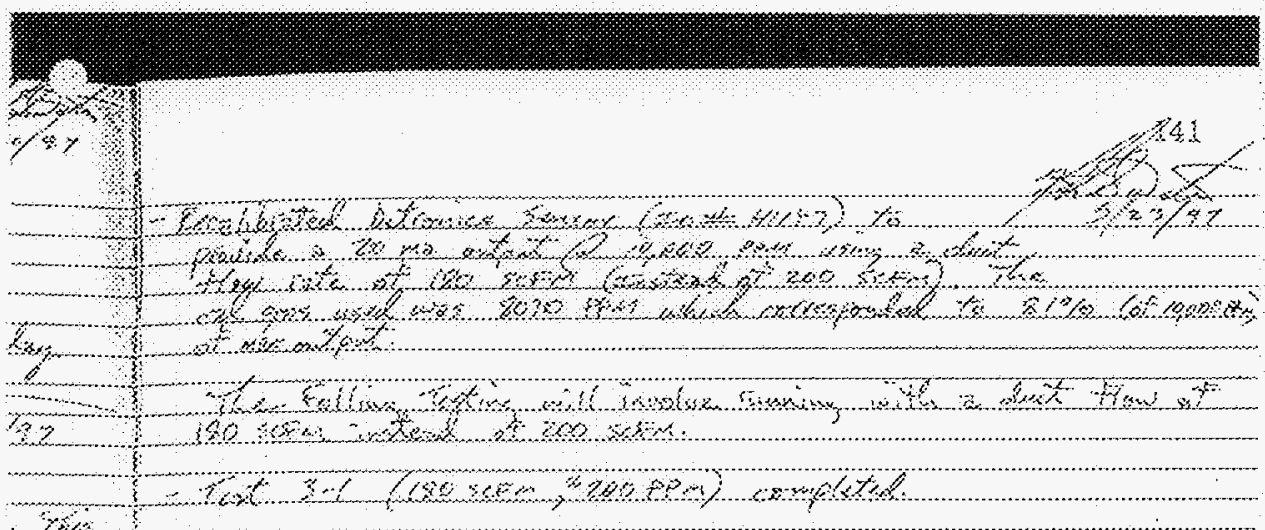

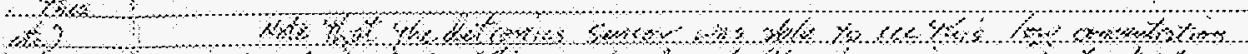

oxom

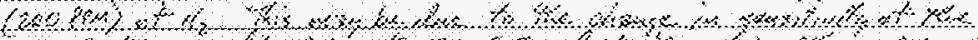

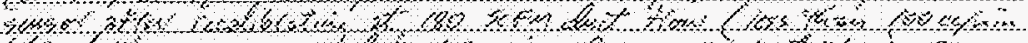

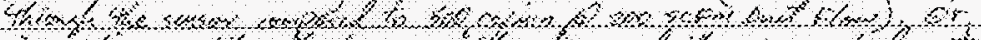

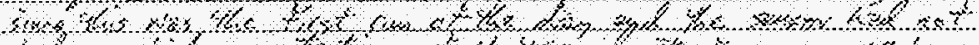

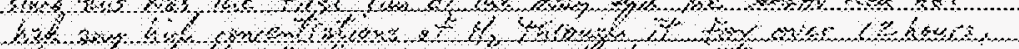

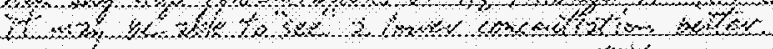

\%

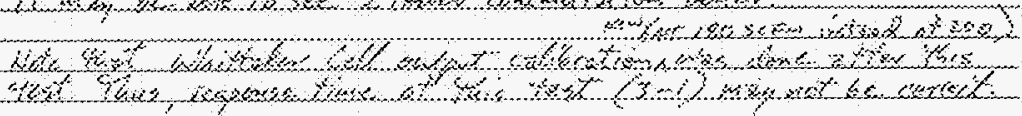

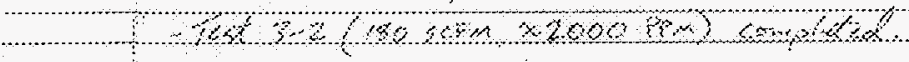

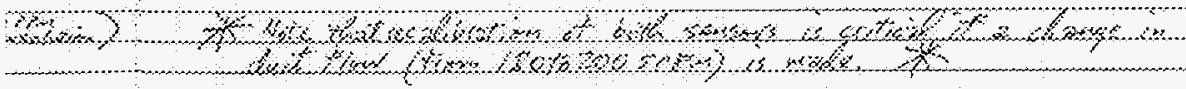

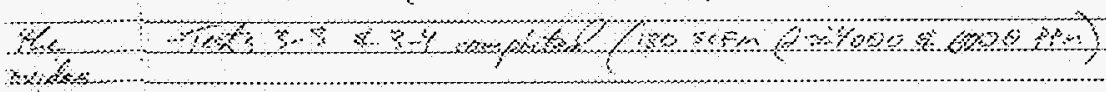
ser.

s.

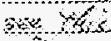

Ho: 
197-55-206-720-266

Kev of

Page is

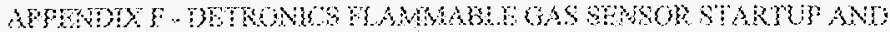

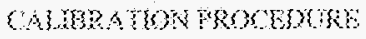




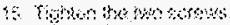

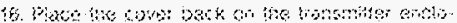
sats.

\section{Mere}

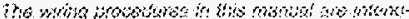

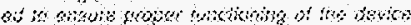

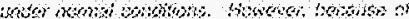

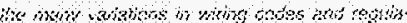

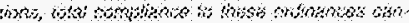

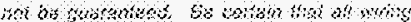

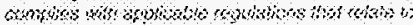

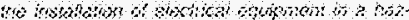

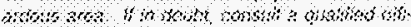
wat bets thot thit syom!

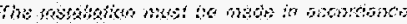
with the set

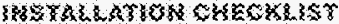

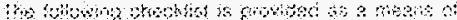

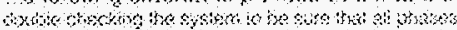

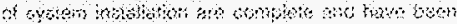
wement montiv

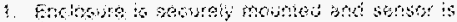
potition

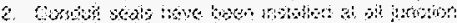

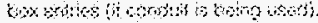

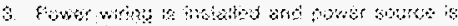
costowis.

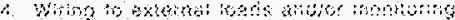

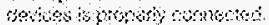

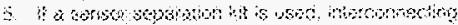
mintosing

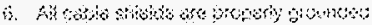

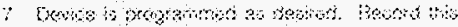

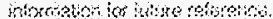

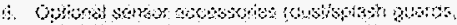

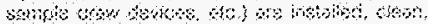
wh ingon onthor

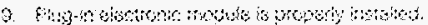

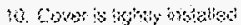

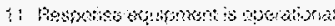

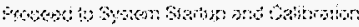

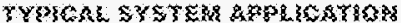

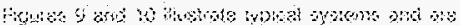

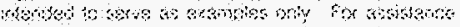

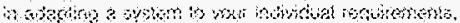

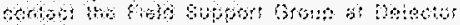
Estrotios

\section{Fection Systers Stantape}

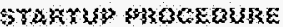

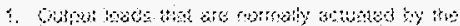

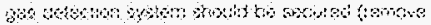

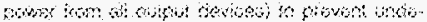

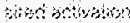

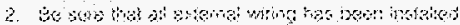

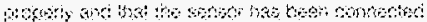

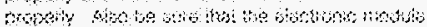

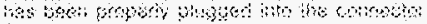

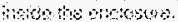

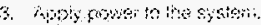

\section{wite:}

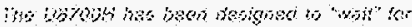

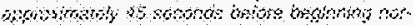

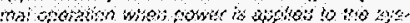

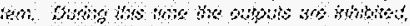

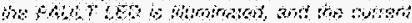

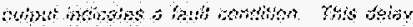

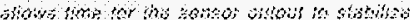

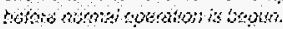

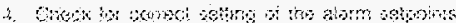

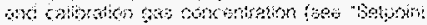

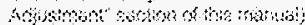

\section{WOTE}

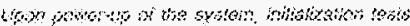

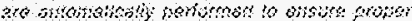

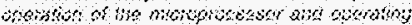

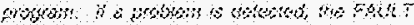

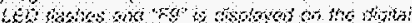

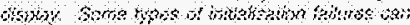

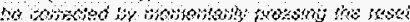

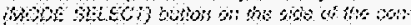

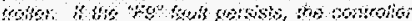

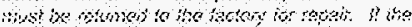

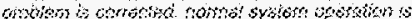

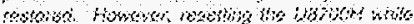

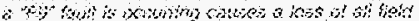




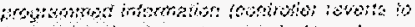

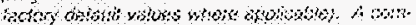

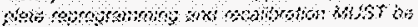

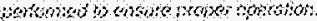

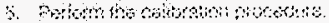

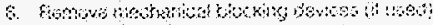

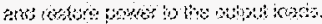

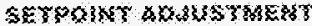

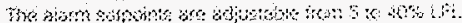

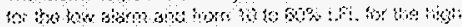

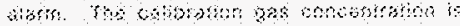

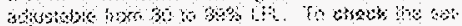

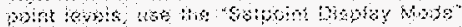

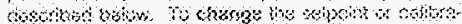

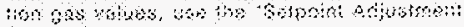
"wotere"

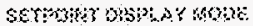

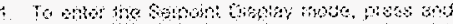

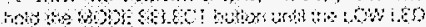

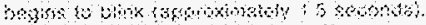

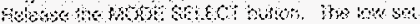

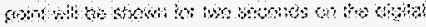
ritosy

\section{bot?}

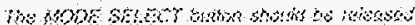

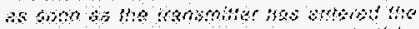

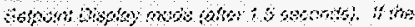

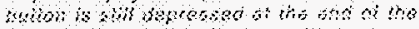

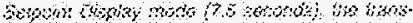

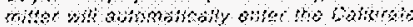

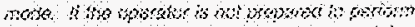

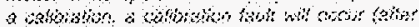
(j) prowtis)

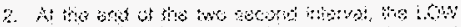

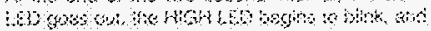

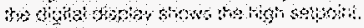

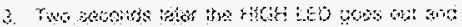

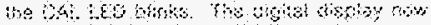

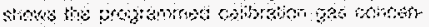
isosicts.

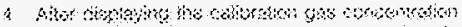

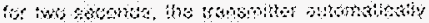

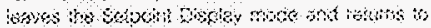

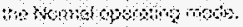

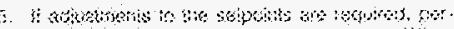

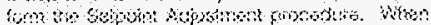

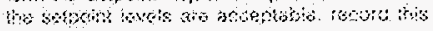

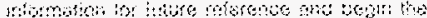

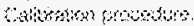

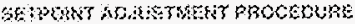

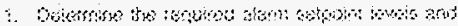

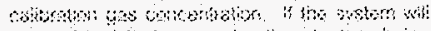

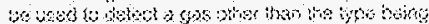

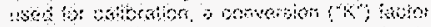

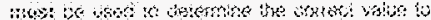

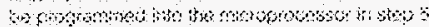

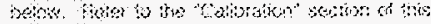
ingthel for botats

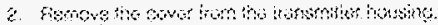

\section{Gastis, 3}

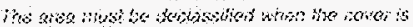

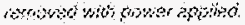

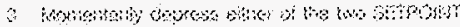

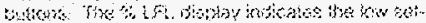

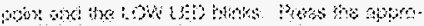

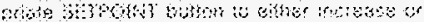

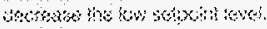

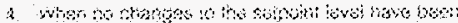

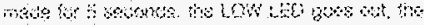

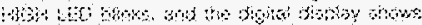

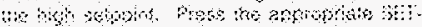

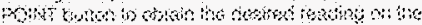

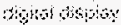

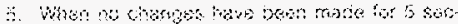

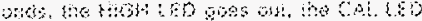

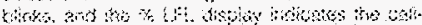

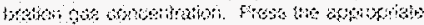

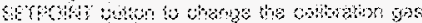

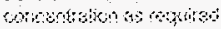

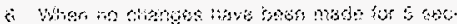

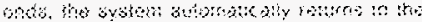

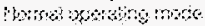

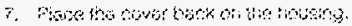

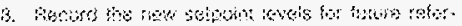
as

wir:

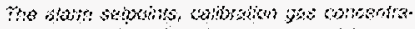

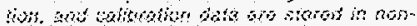

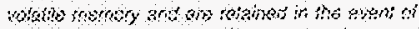

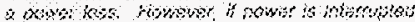

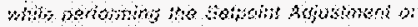

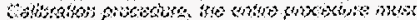

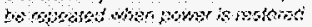




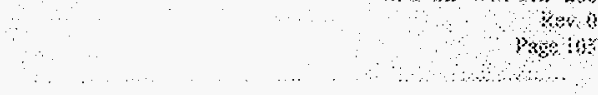

\section{CaLsot3insose}

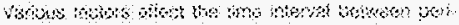

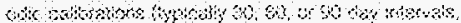

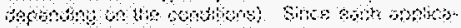

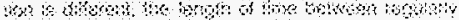

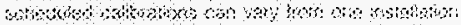

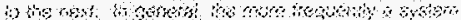

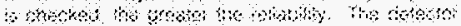

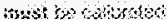

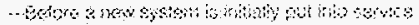

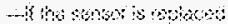

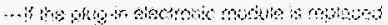

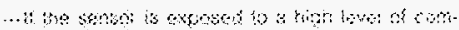
notberos.

\section{CABTKN}

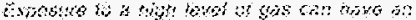

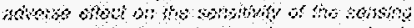

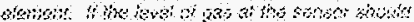

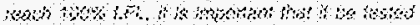

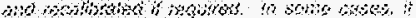

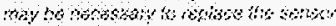

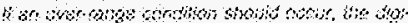

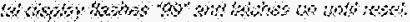

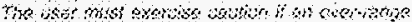

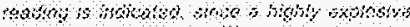

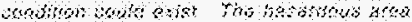

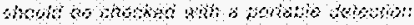

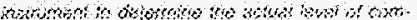

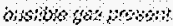

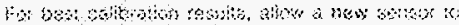

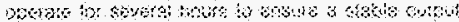

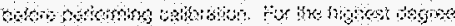

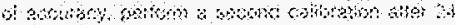
hotis.

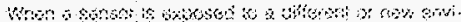

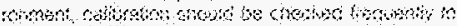

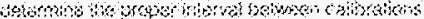

\section{wrs}

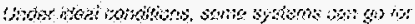

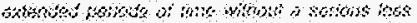

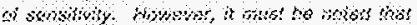

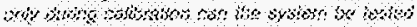

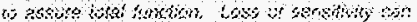

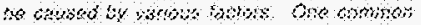

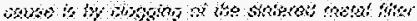

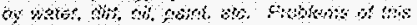

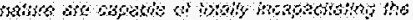

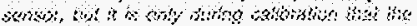

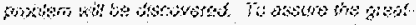

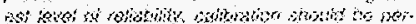

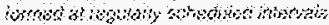

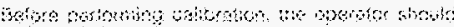

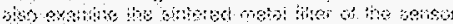

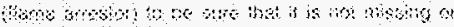

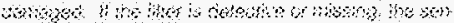

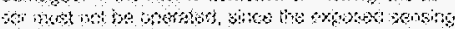

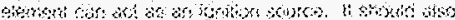

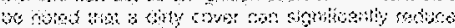

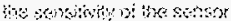

\section{woit}

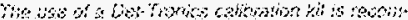

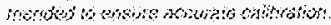

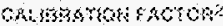

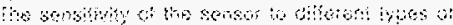

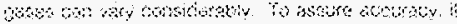

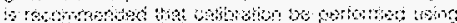

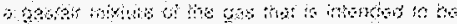

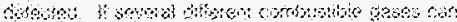

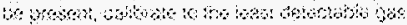

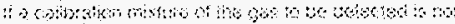

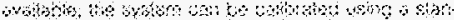

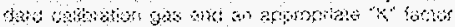

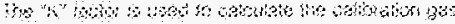

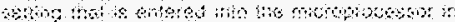

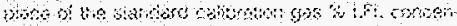

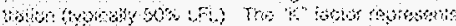

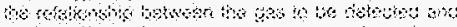

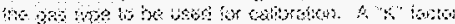

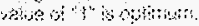

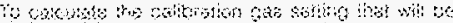

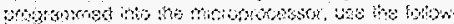
mistomisa

$$
5=0: \div
$$

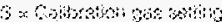

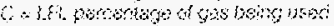

$$
\begin{aligned}
& \therefore \because \text { Coturabo beth }
\end{aligned}
$$

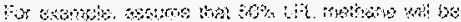

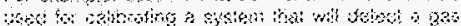

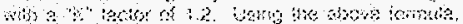

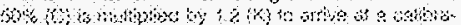

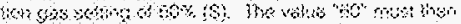

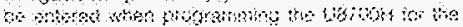
tatomontos

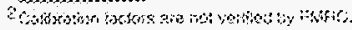




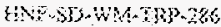

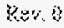

Prge : $3 \%$

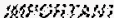

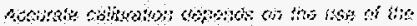

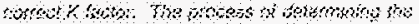

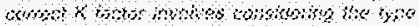

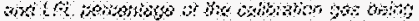

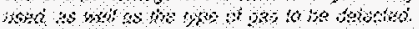

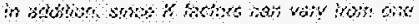

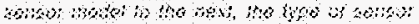

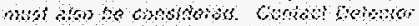

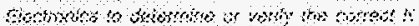

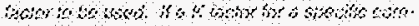

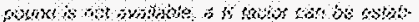

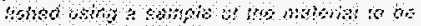

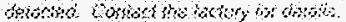

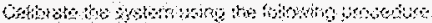

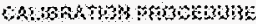

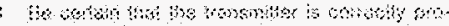

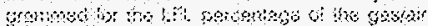

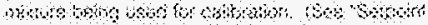

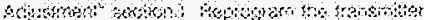

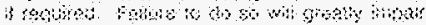
systit reopose.

\section{wis:}

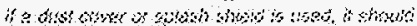

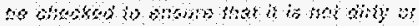

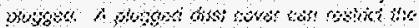

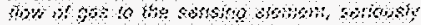

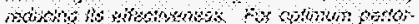

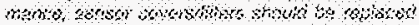

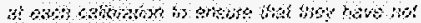

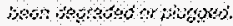

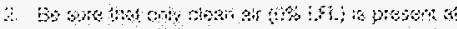

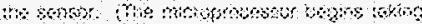

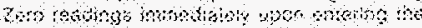

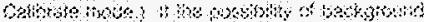

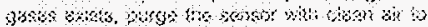

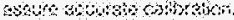

\section{Wi2}

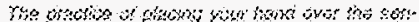

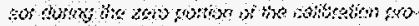

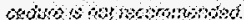

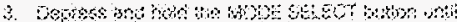

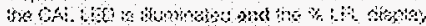

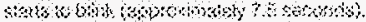

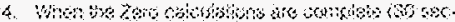

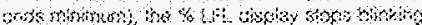
ardestes "h,

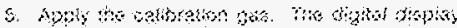

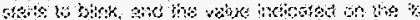

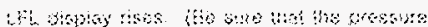

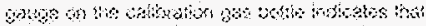

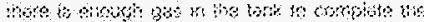
orthation:

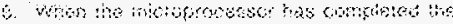

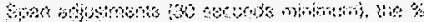

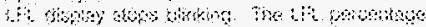

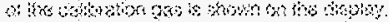

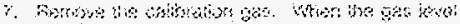

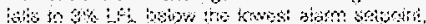

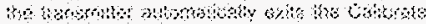

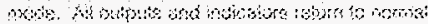
shratio.

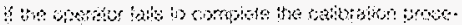

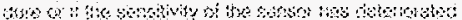

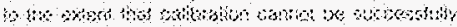

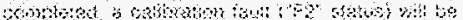

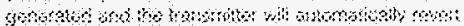

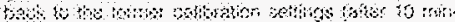

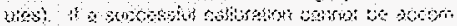

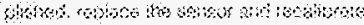

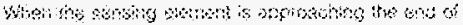

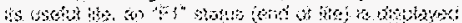

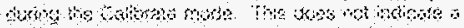

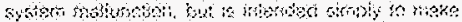

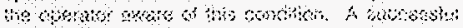

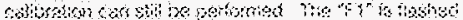

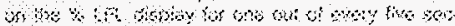

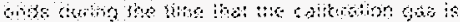

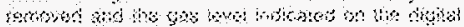

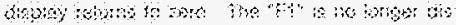

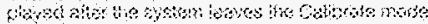

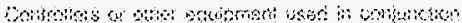

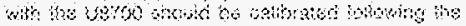

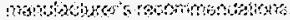

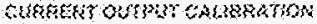

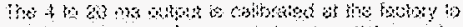

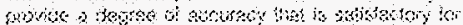

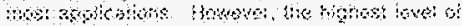

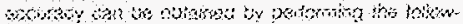
watoristo

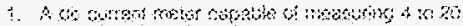

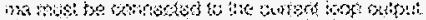

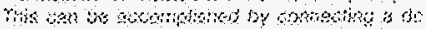

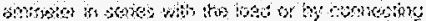

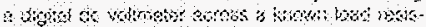

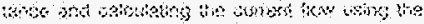
igrensit:

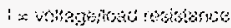




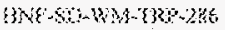

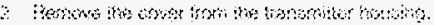

\section{YADTKN}

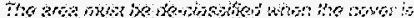
wither wht wow andst.

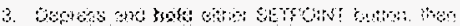

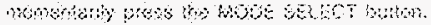

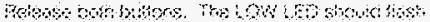

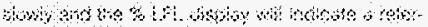

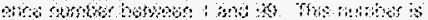

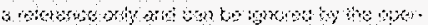

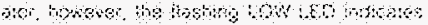

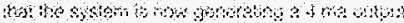

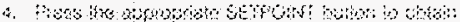

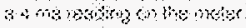

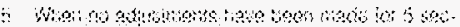

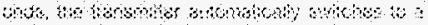

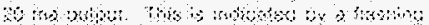

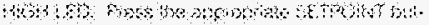

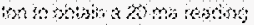

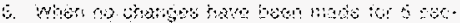

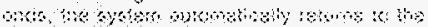

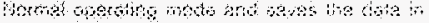

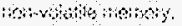

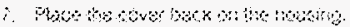

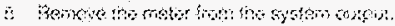

\section{Siecton 8 S

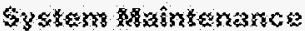

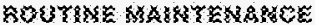

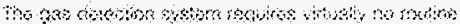

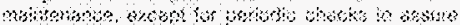

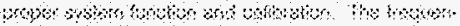

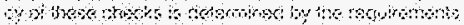

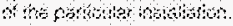

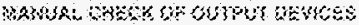

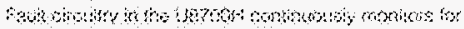

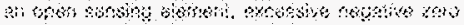

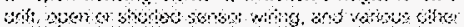

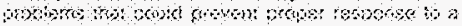

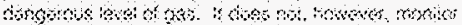

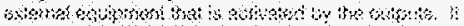

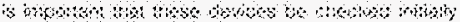

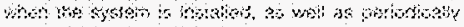

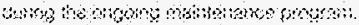

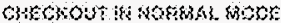

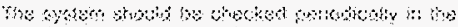

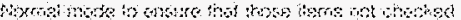

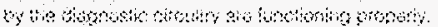

\section{crimor}

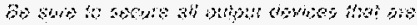

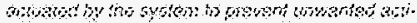

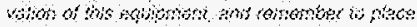

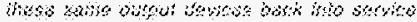
who be wewom is comples

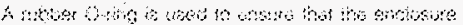

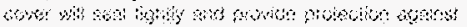

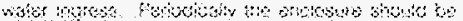

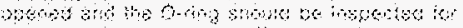

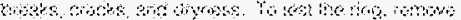

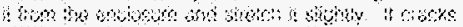

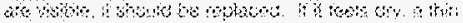

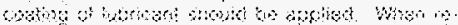

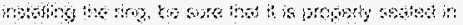

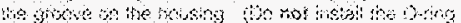

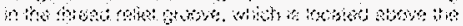

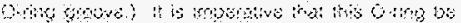

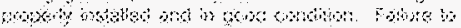

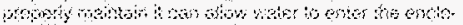

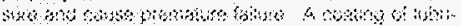

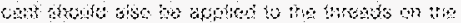

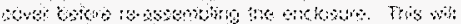

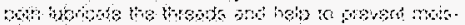

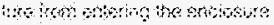

\section{castis?}

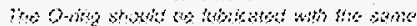

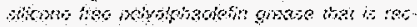

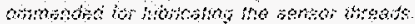

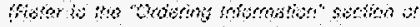

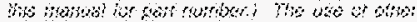

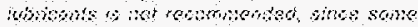

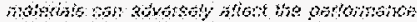

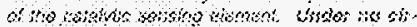

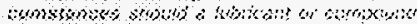
whiting shope be net.

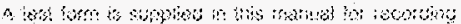

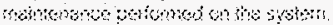

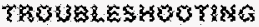

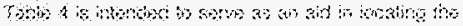

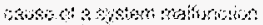

Arit:

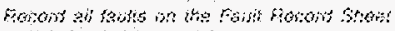

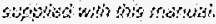




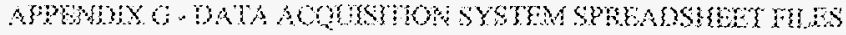




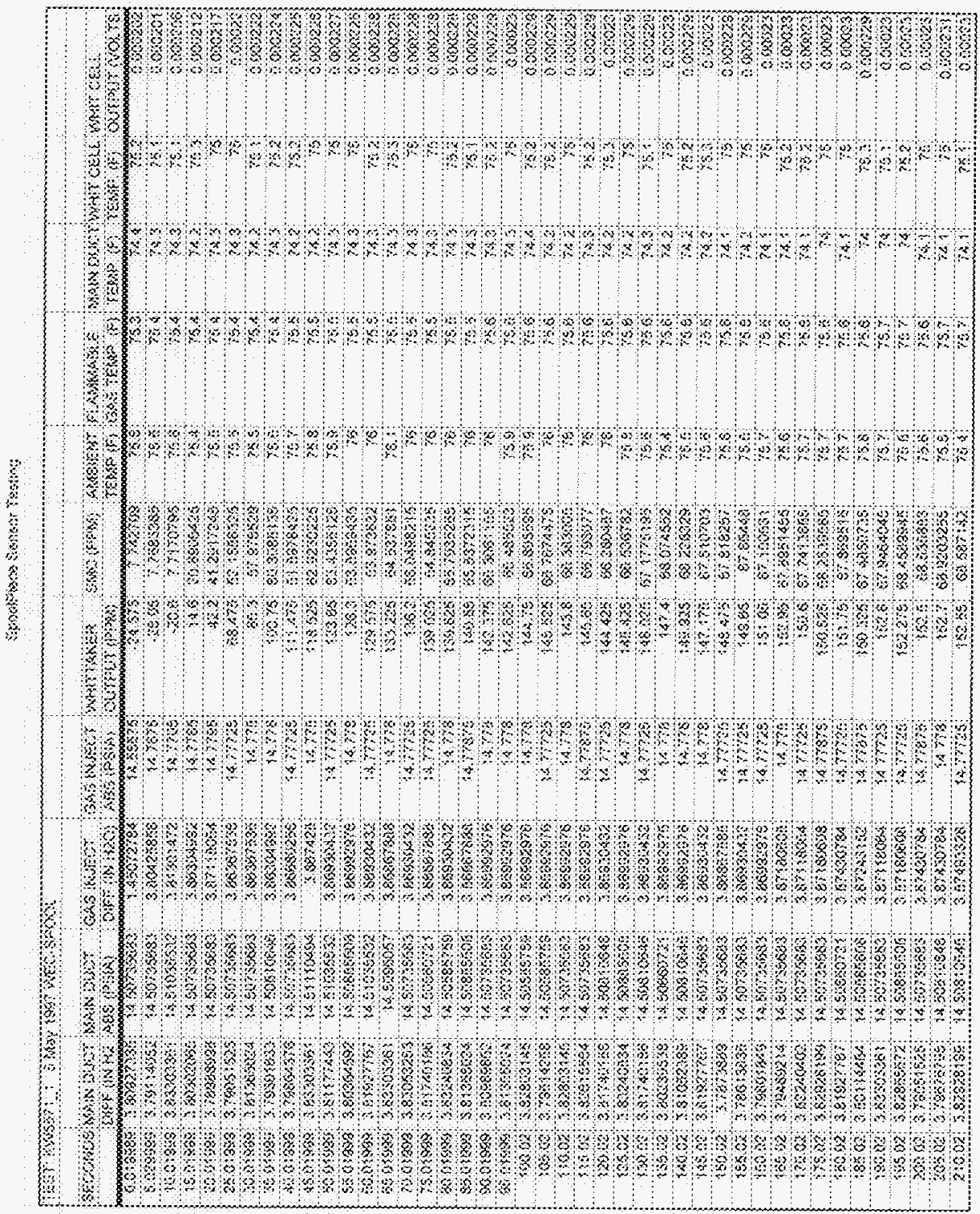




\section{\% $696 \%$}

柿

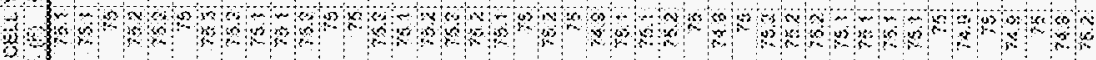
然

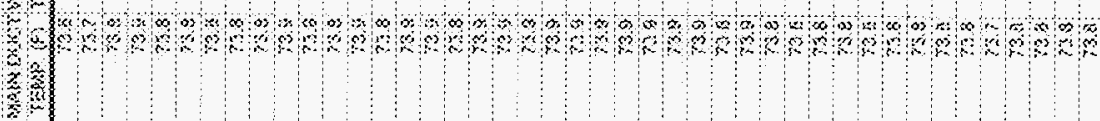
a 3

How was

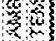

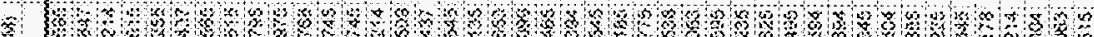

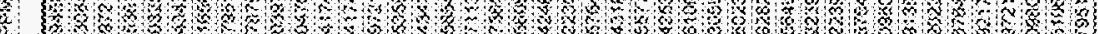

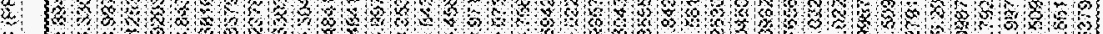

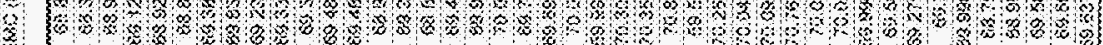

:

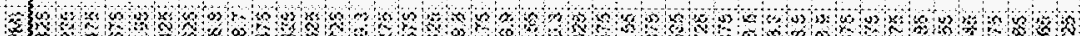

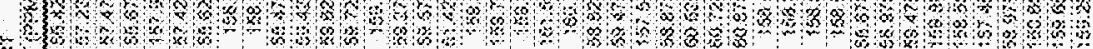

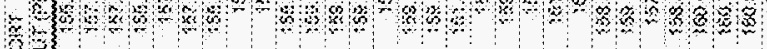

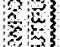

(4)

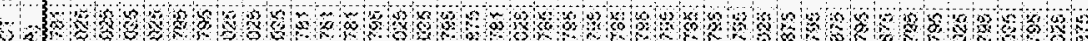

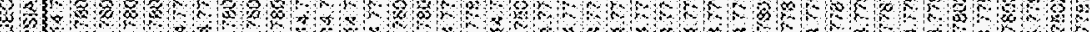

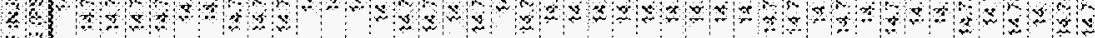

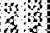

6\% a

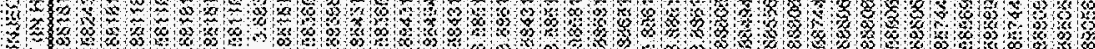

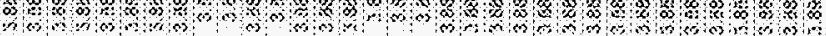
$\$$

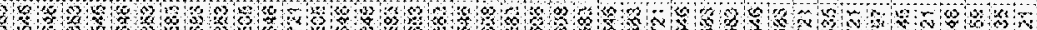

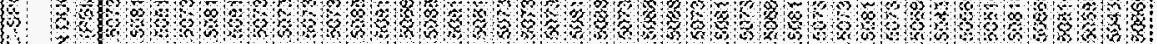
13

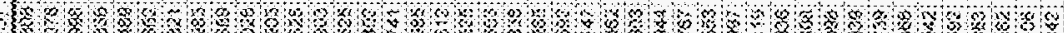

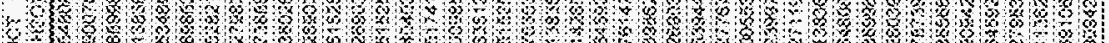

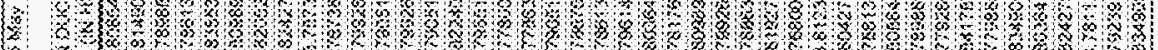

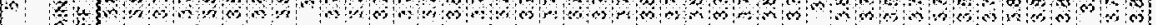
H

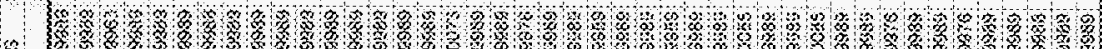

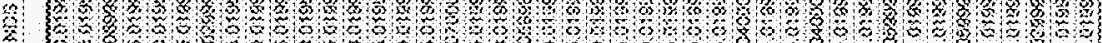

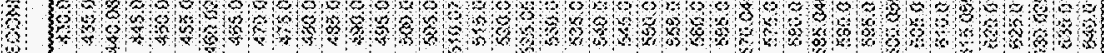




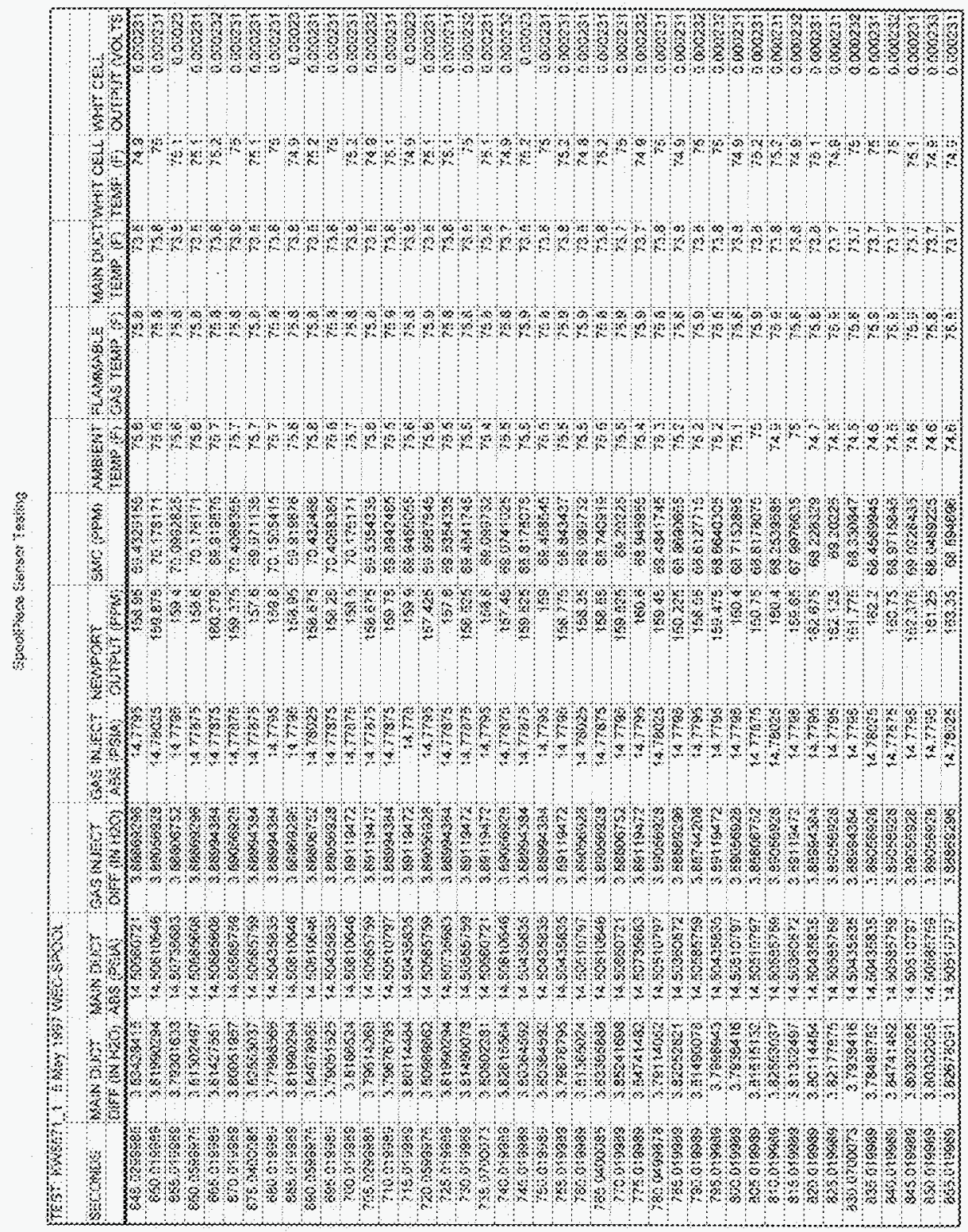




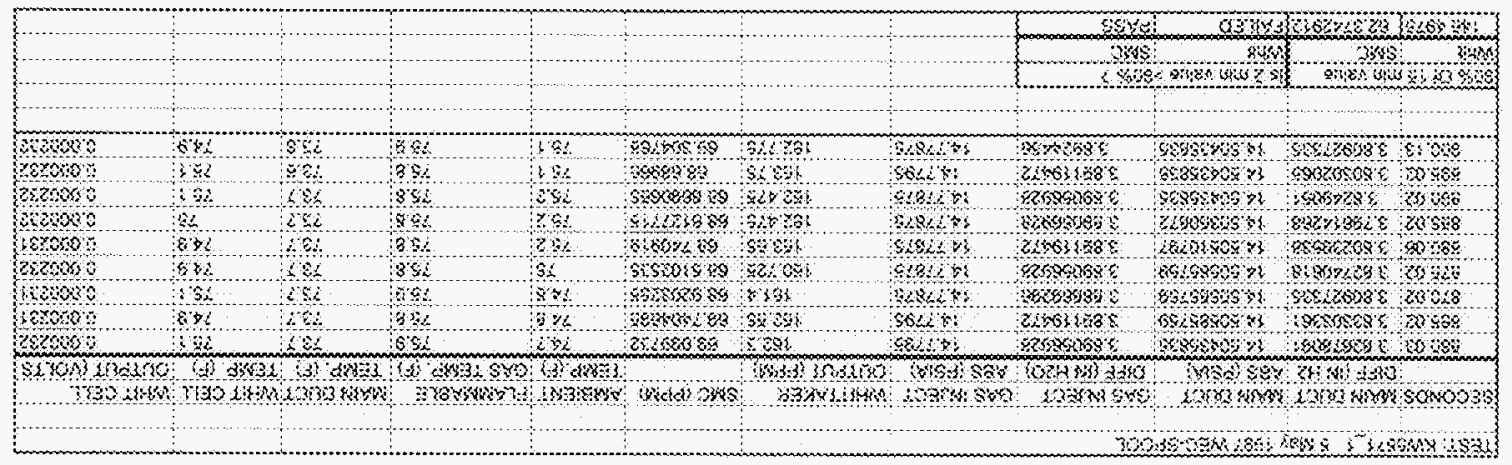




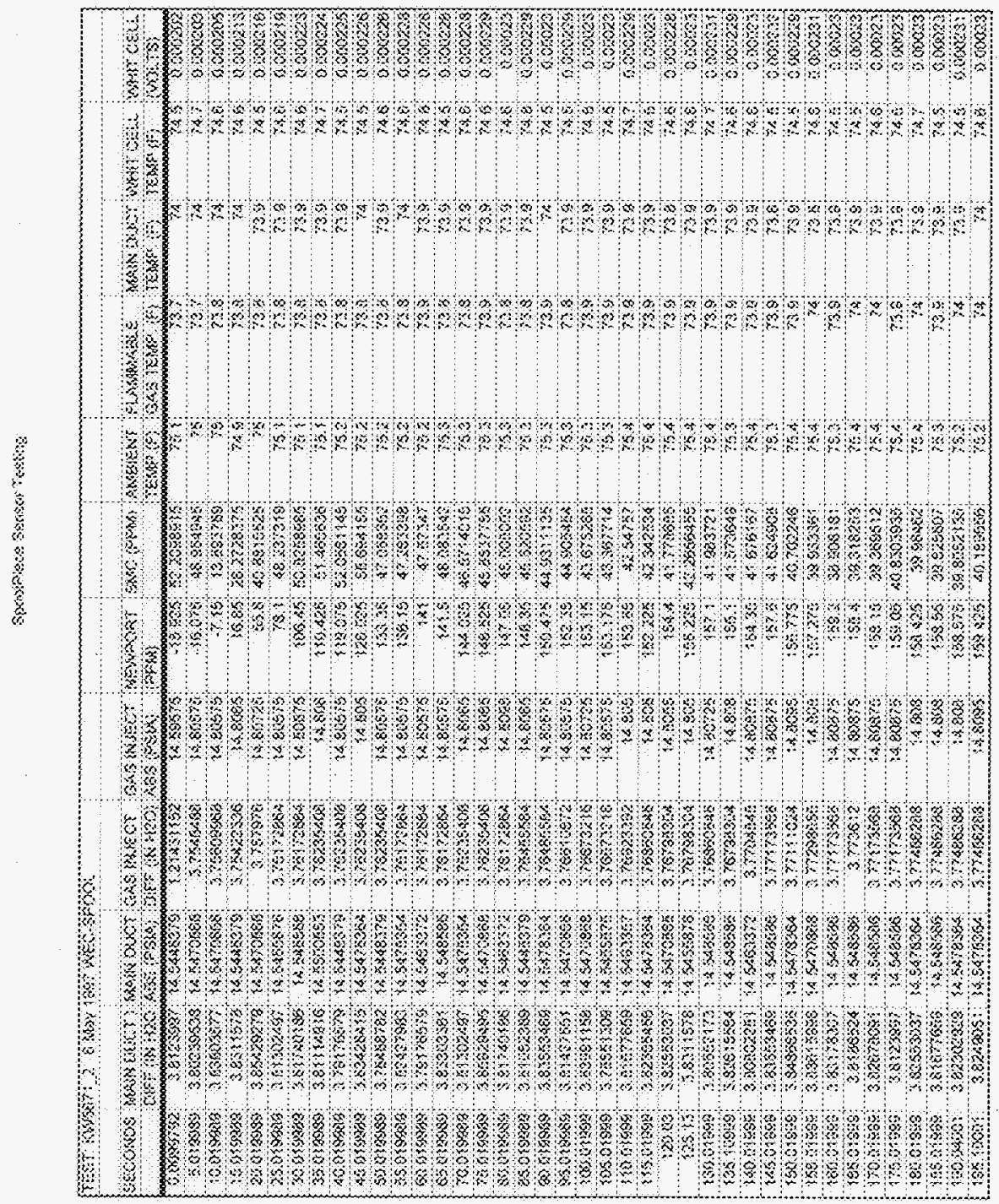




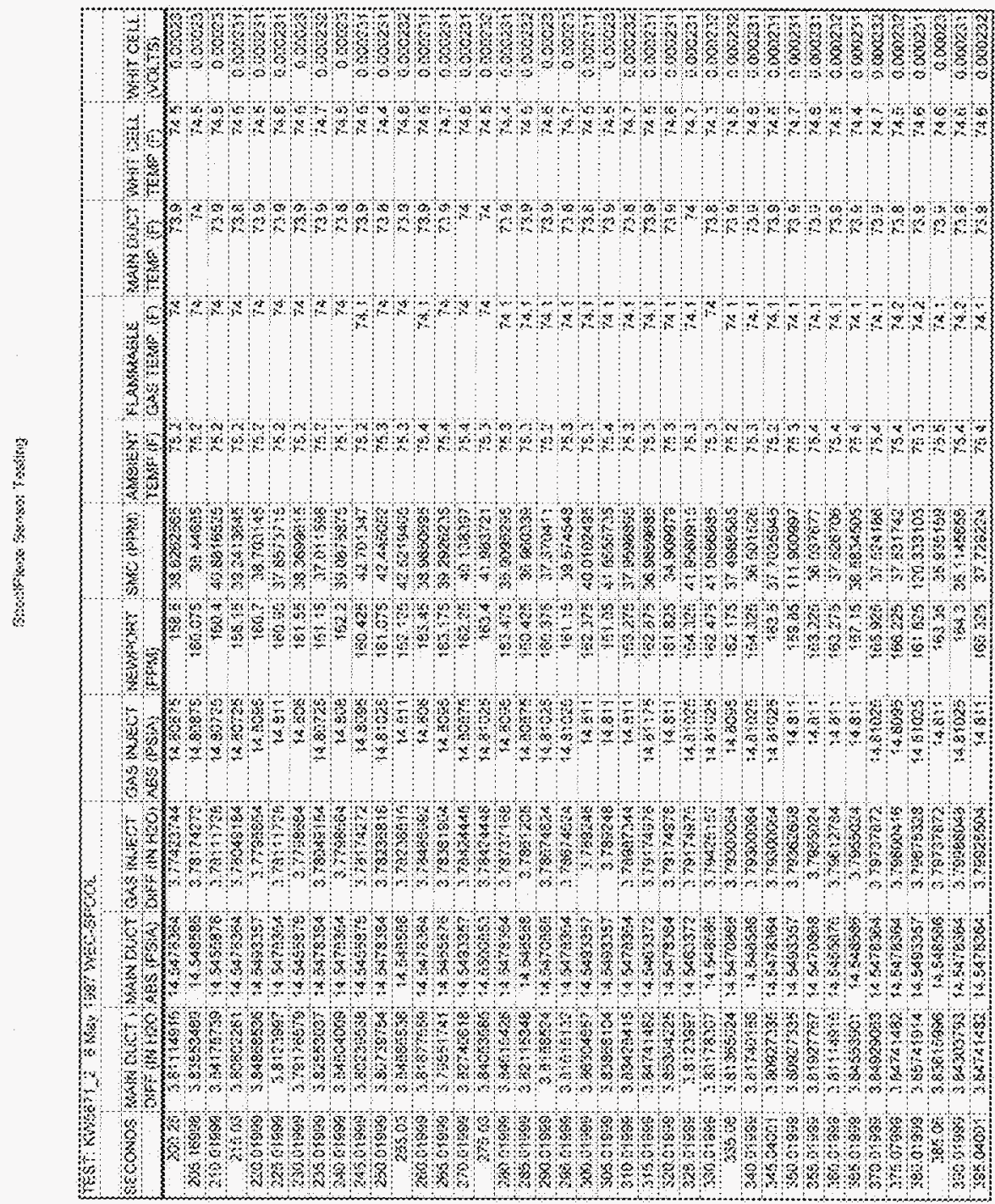




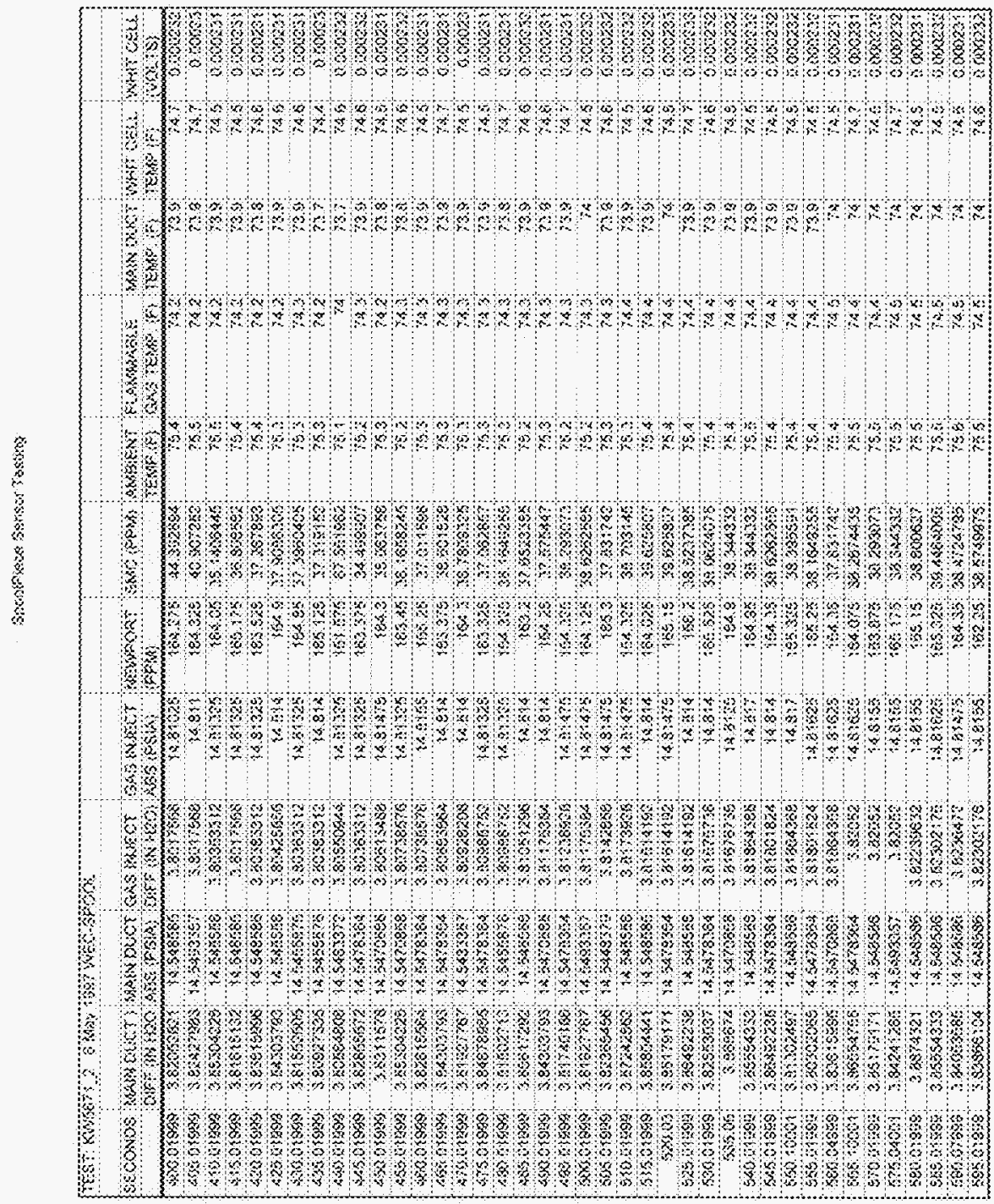




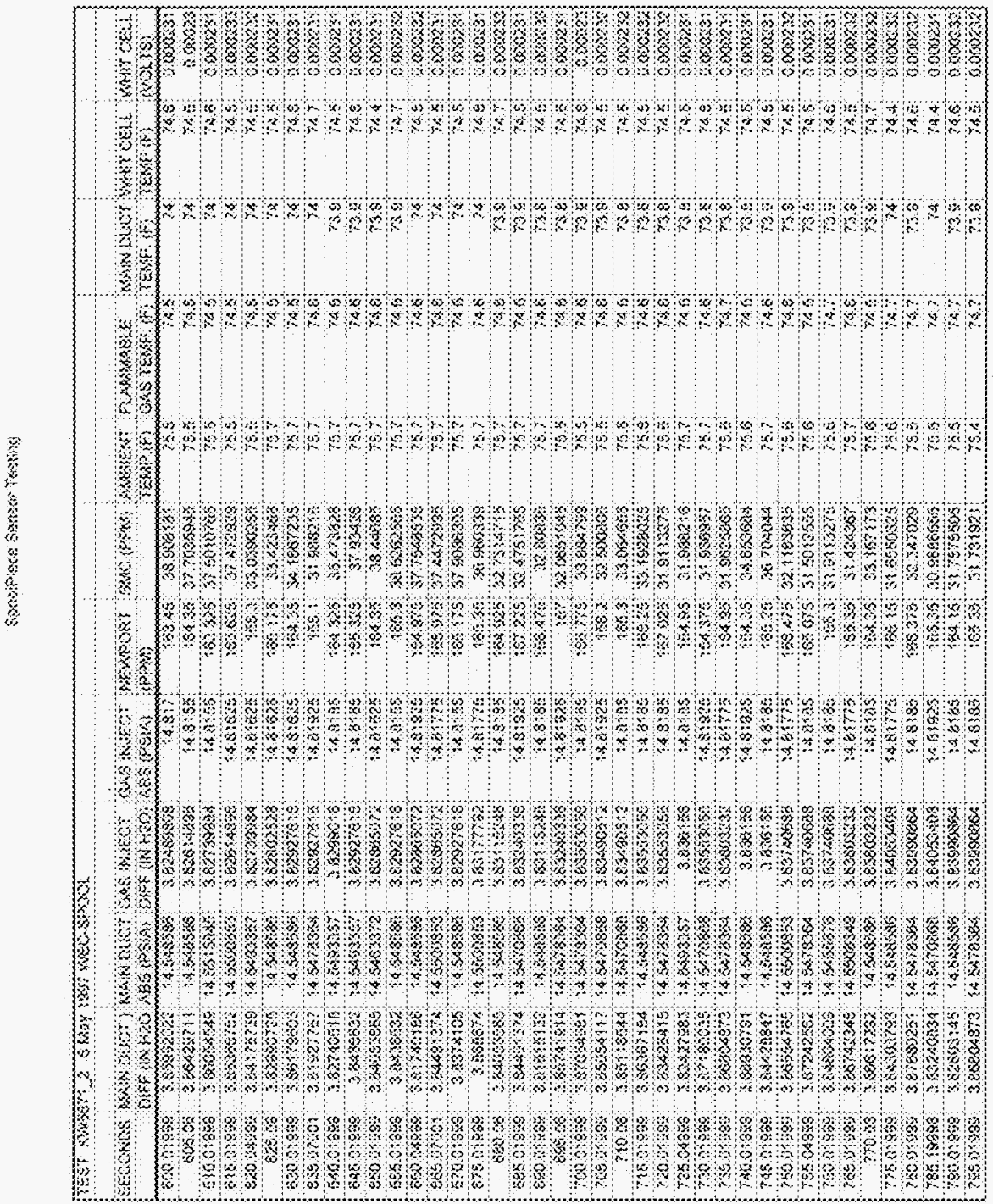




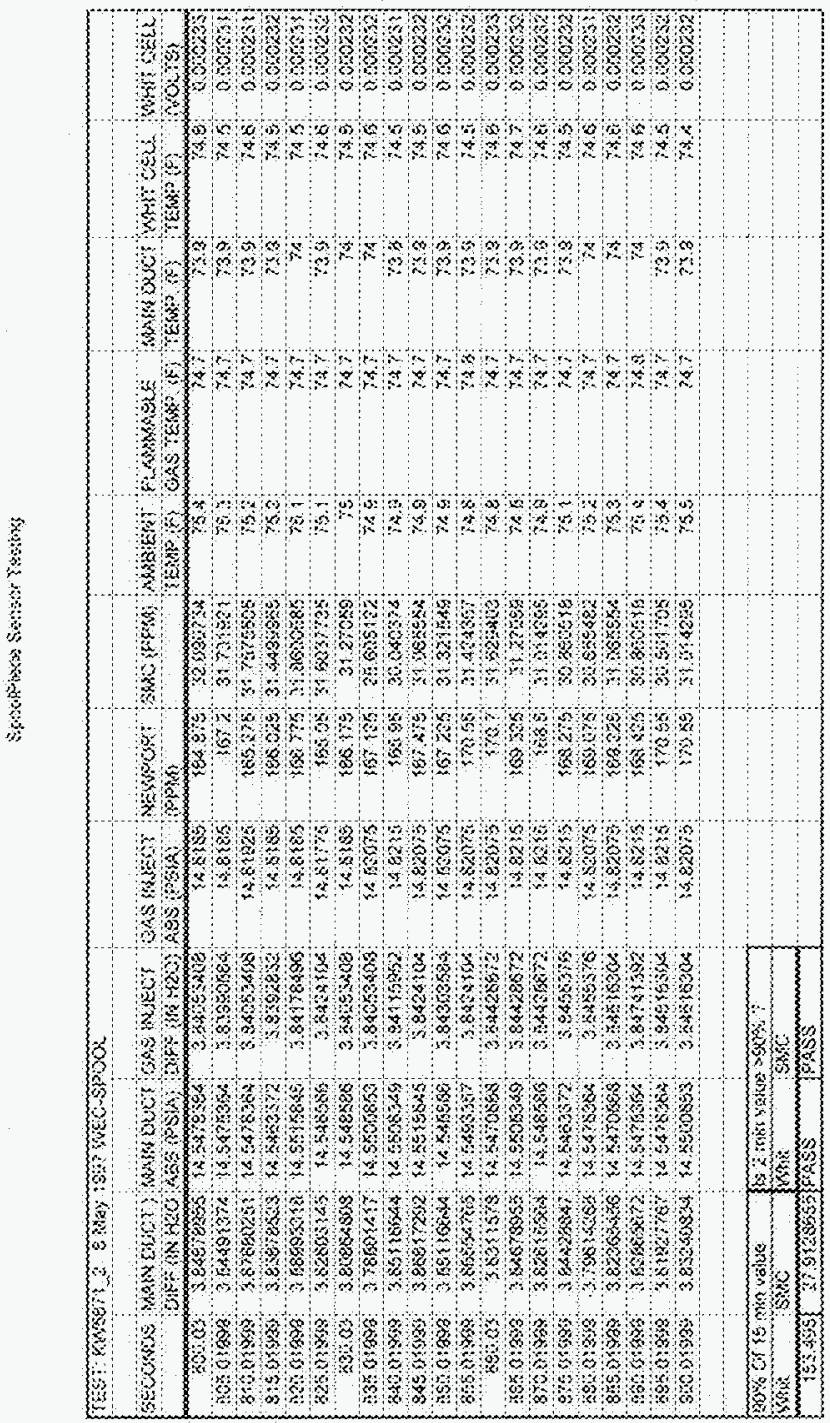




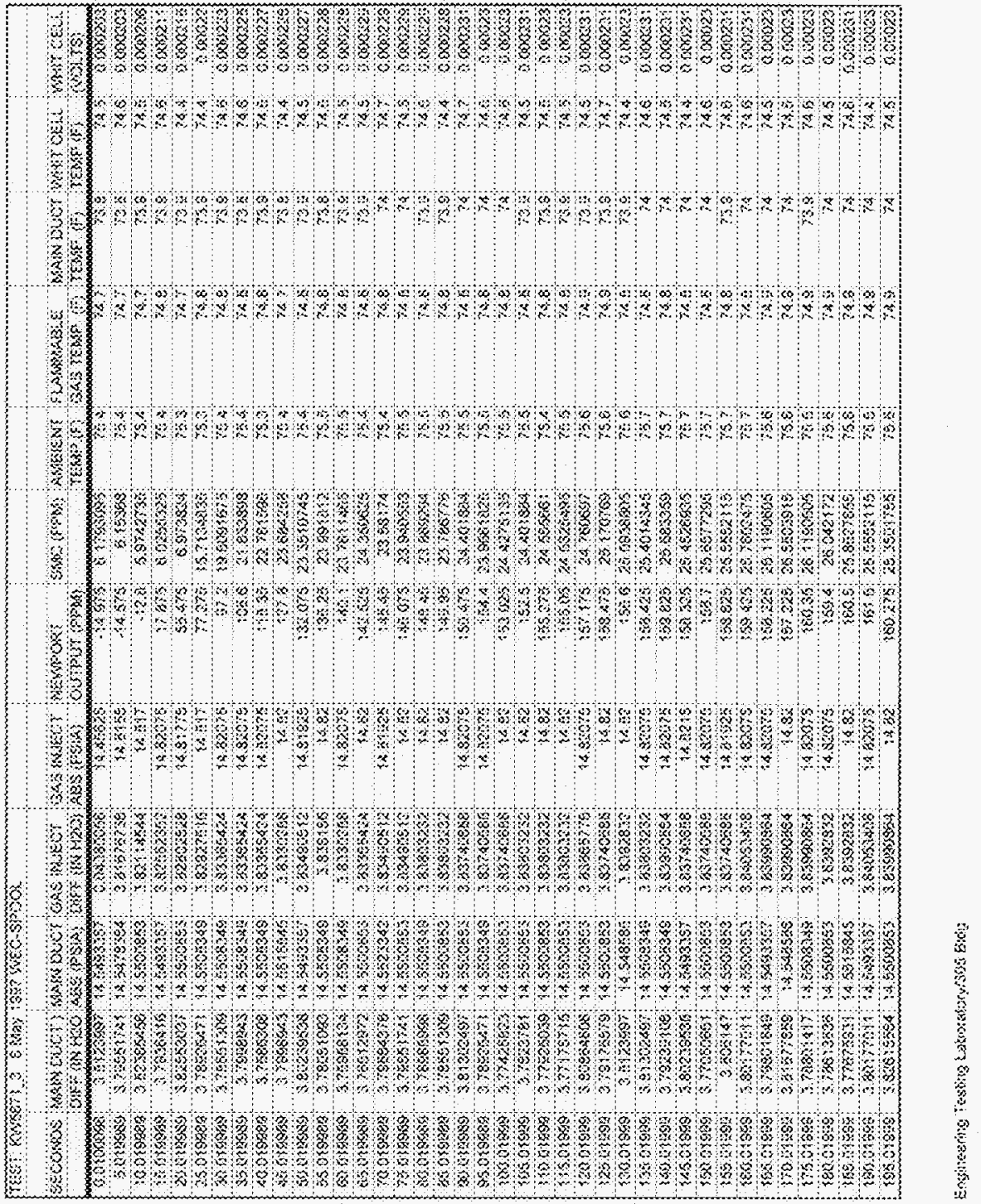




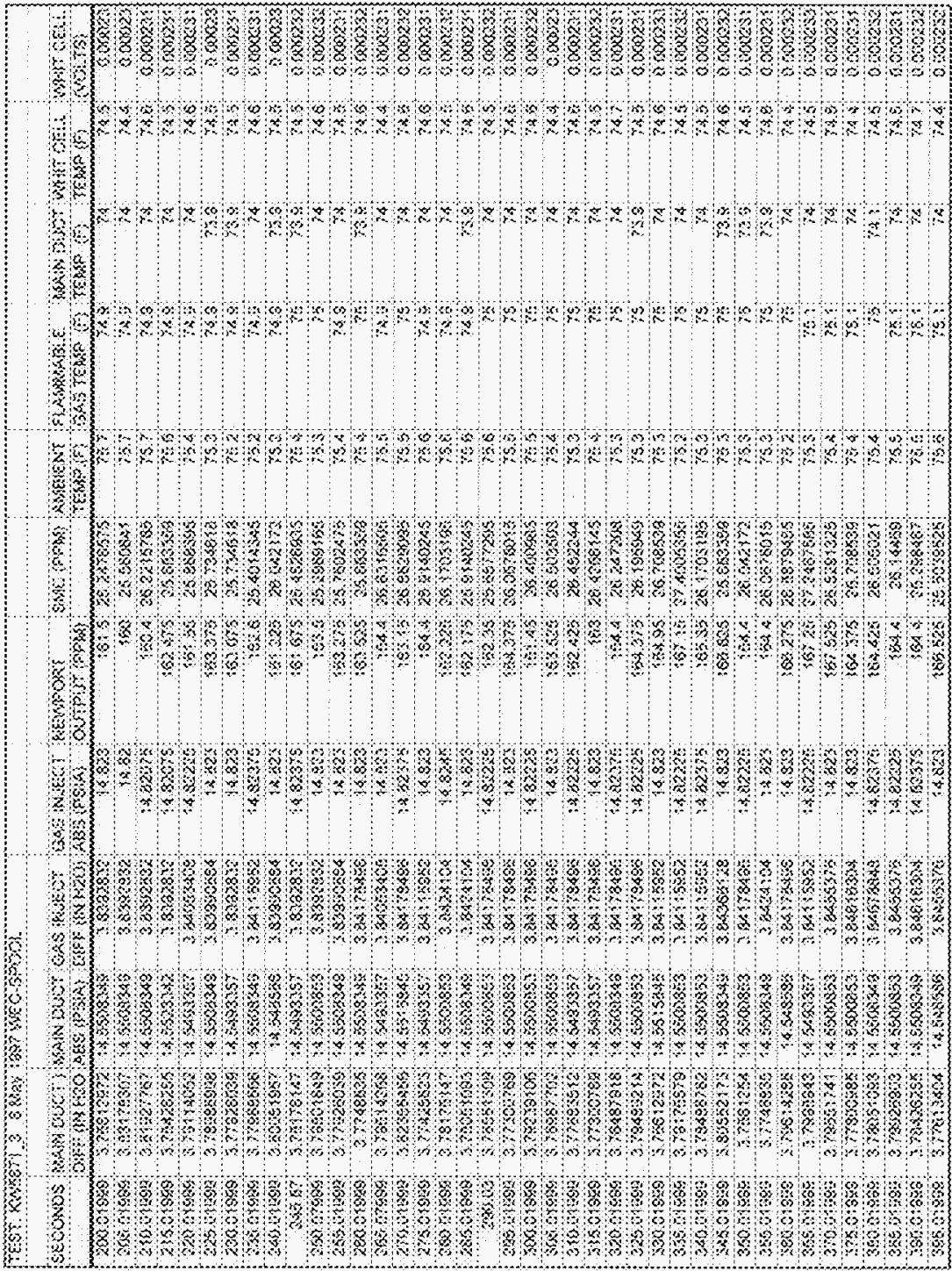




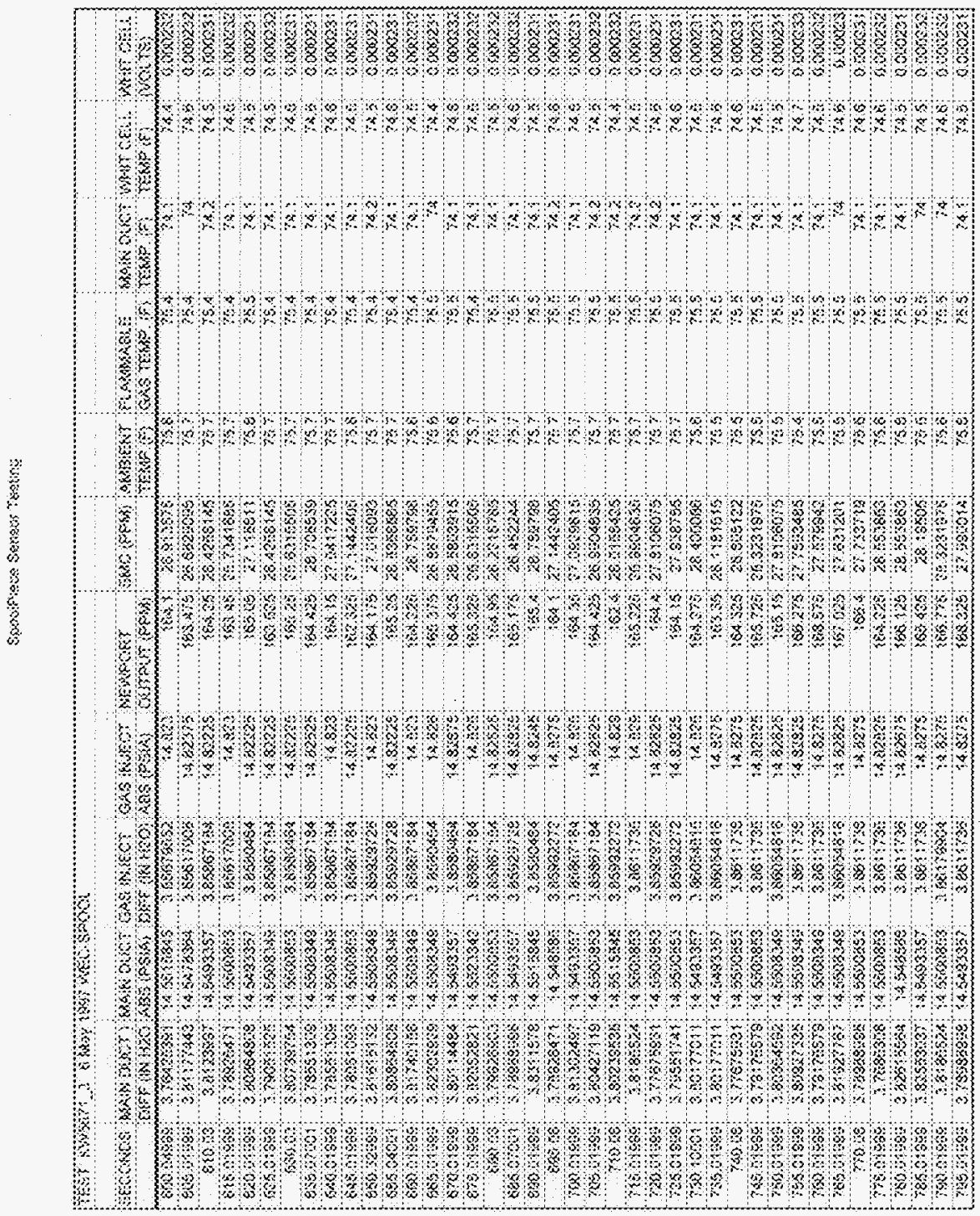




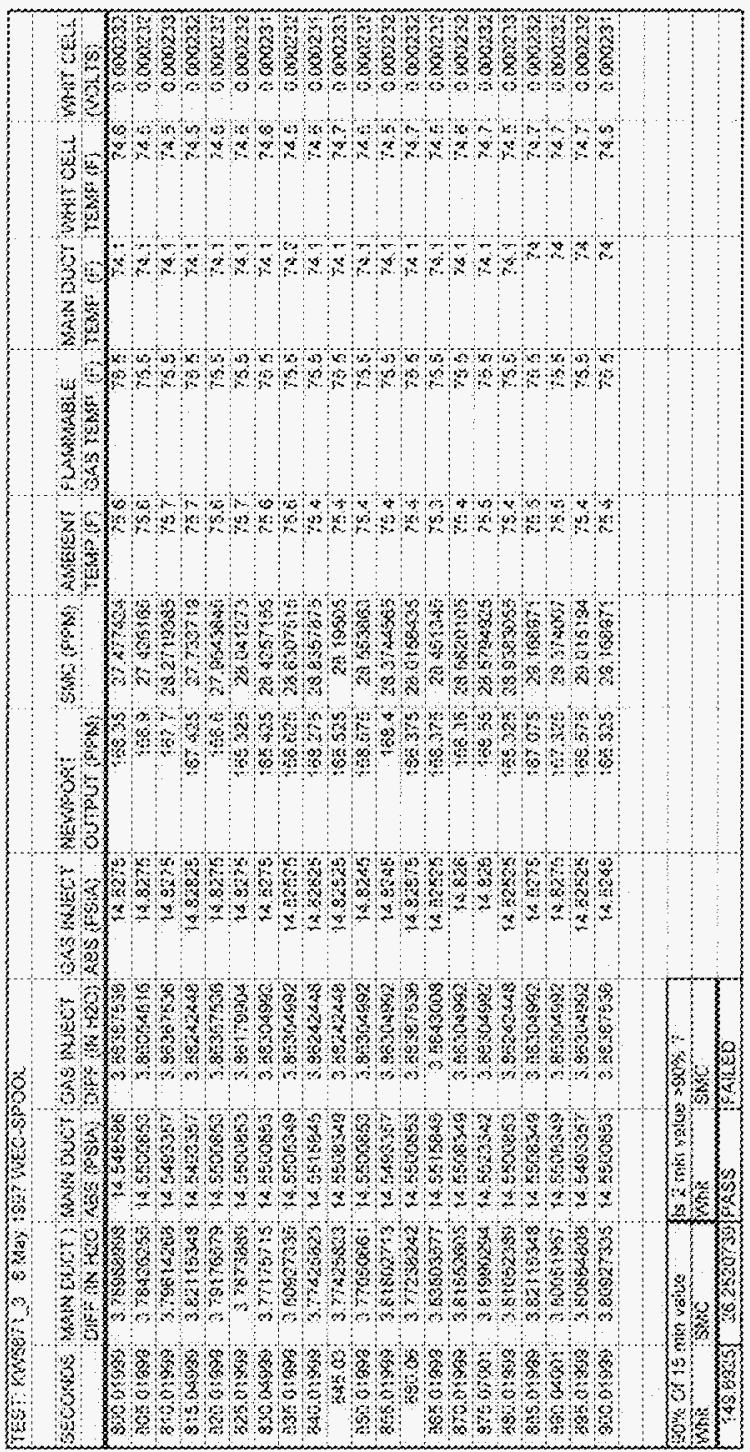




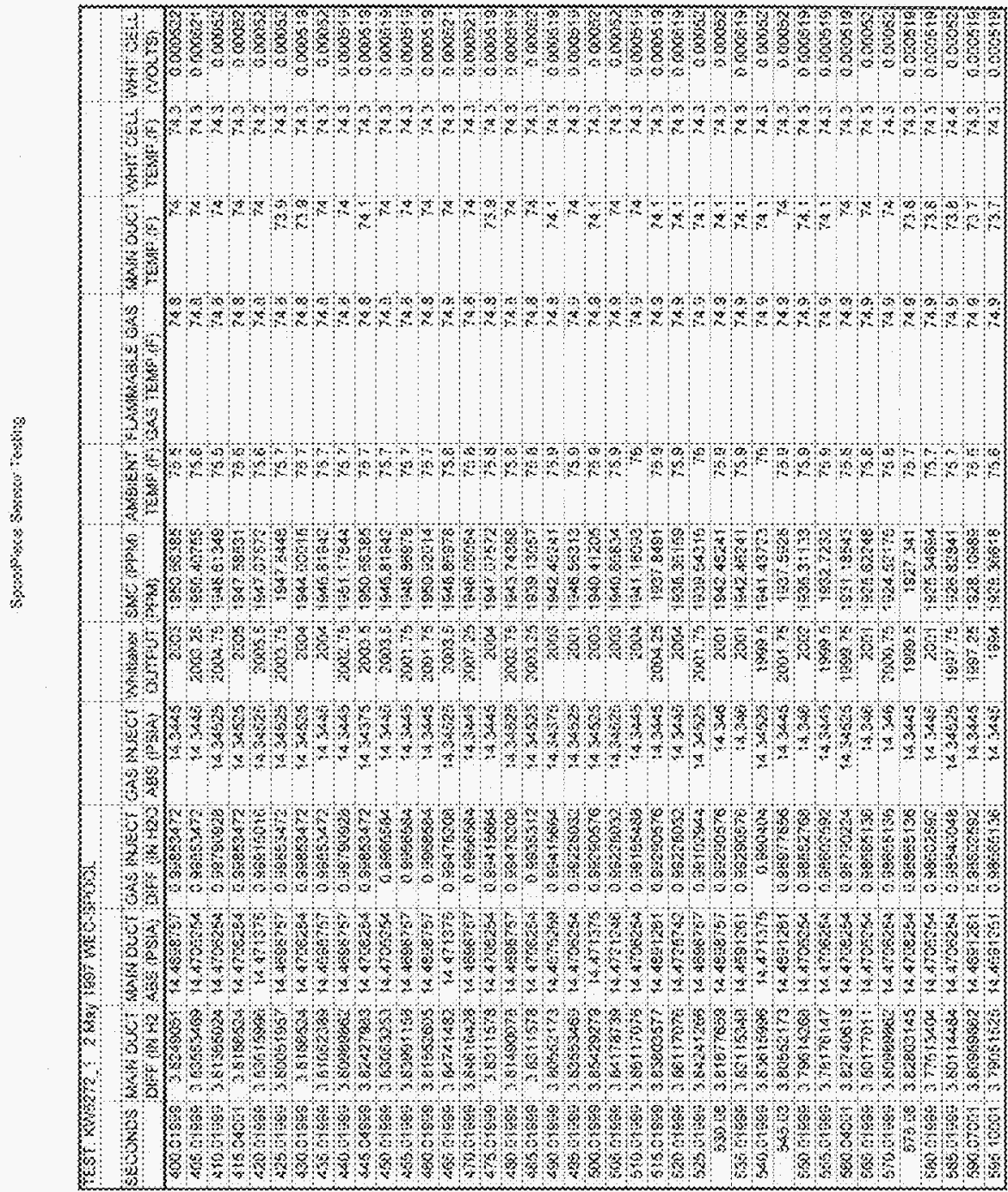




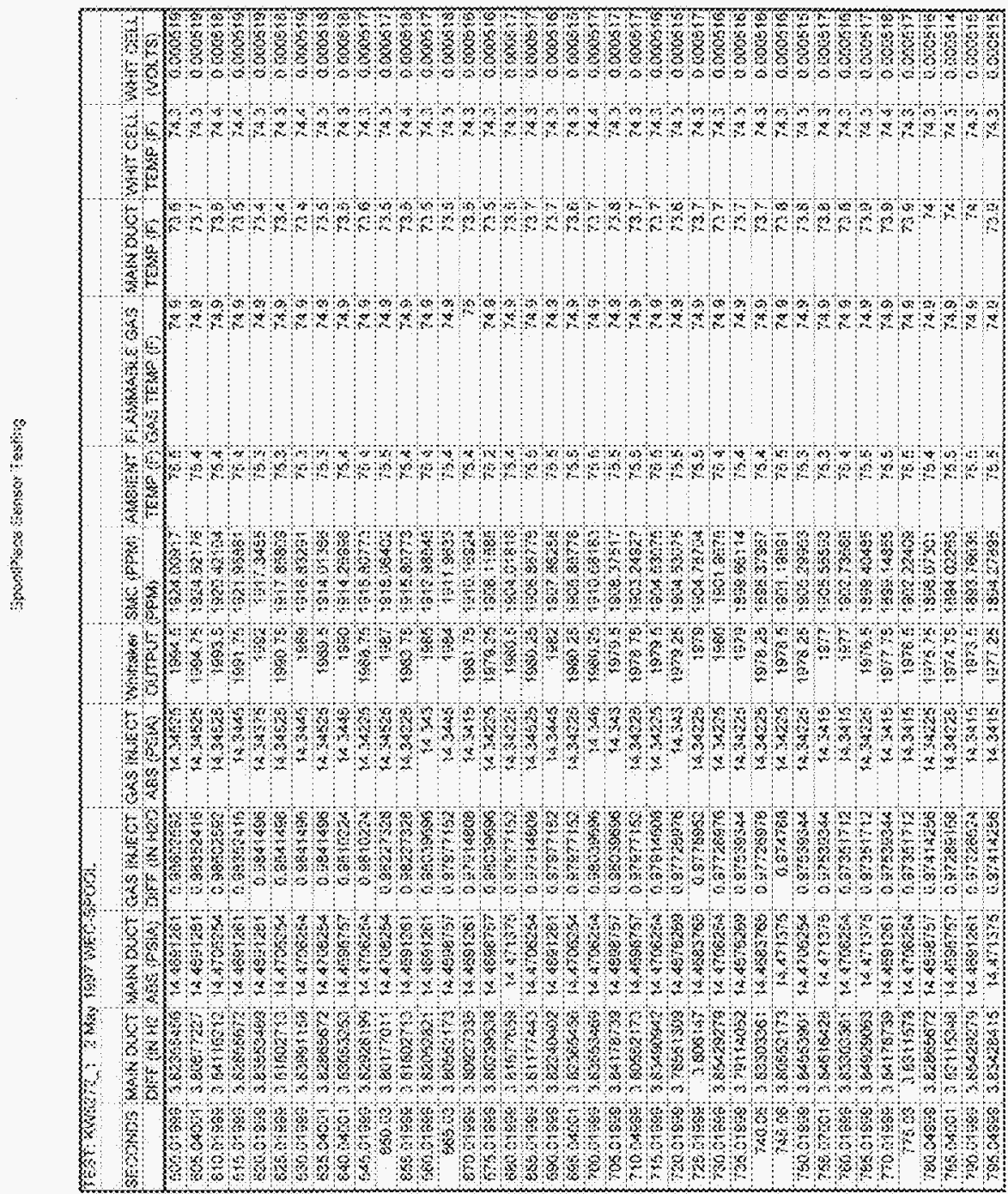




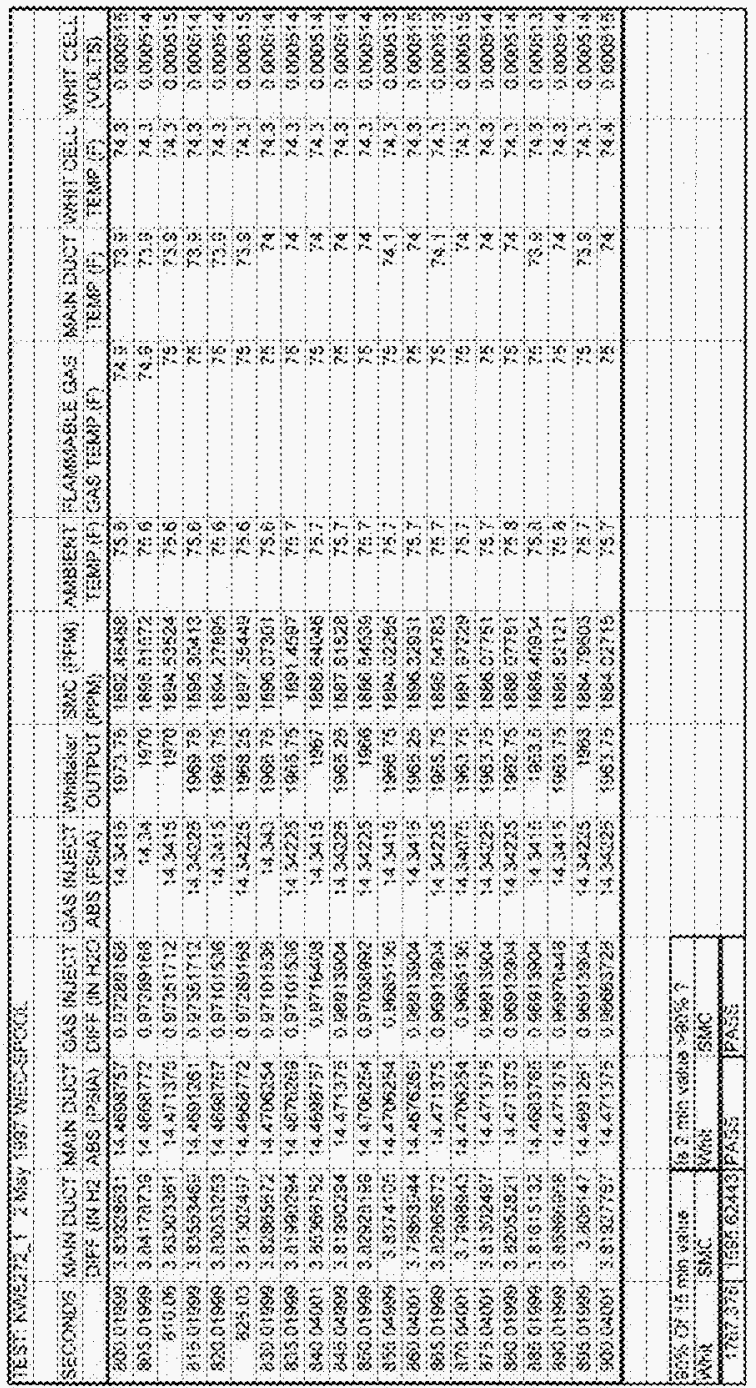




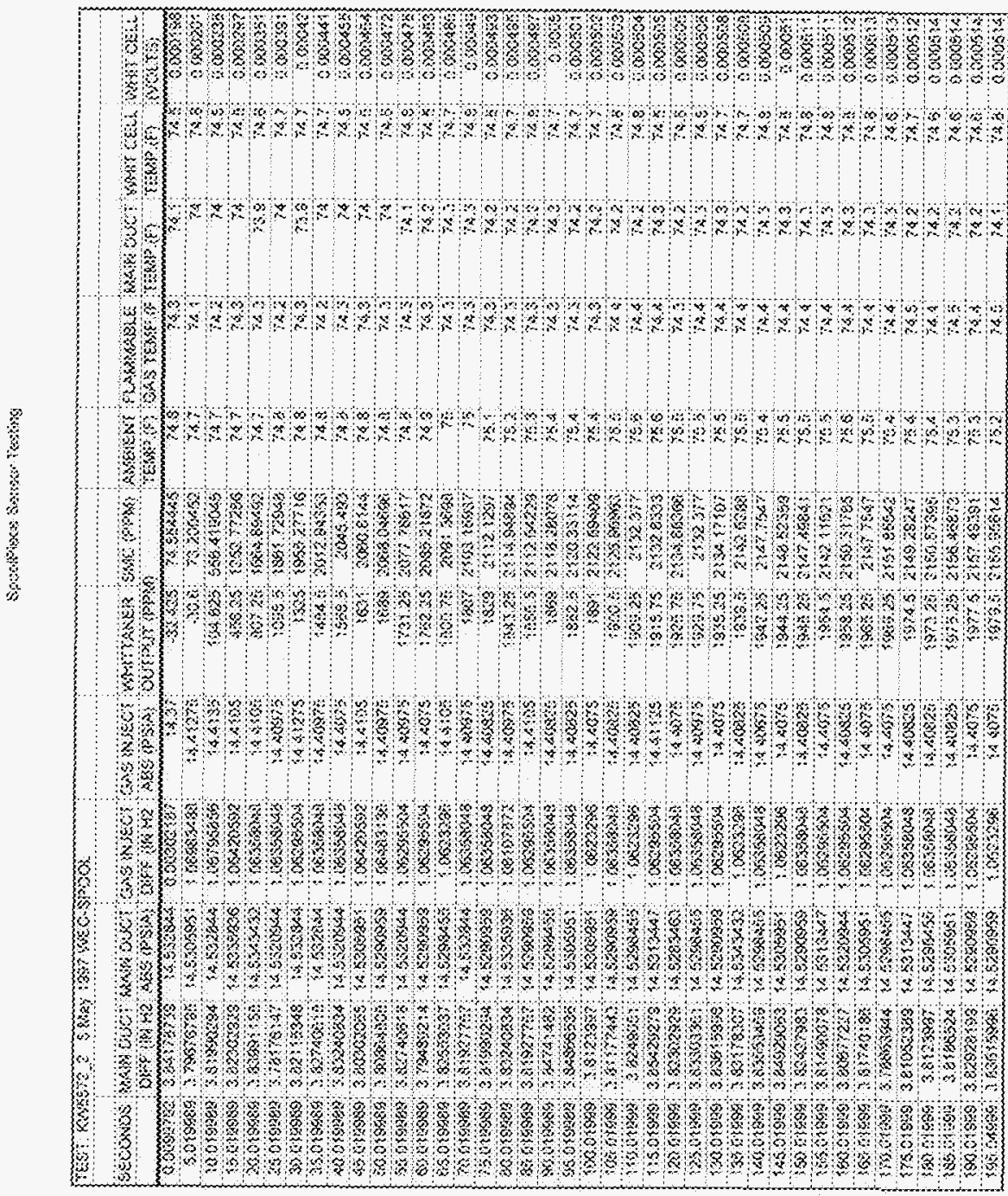




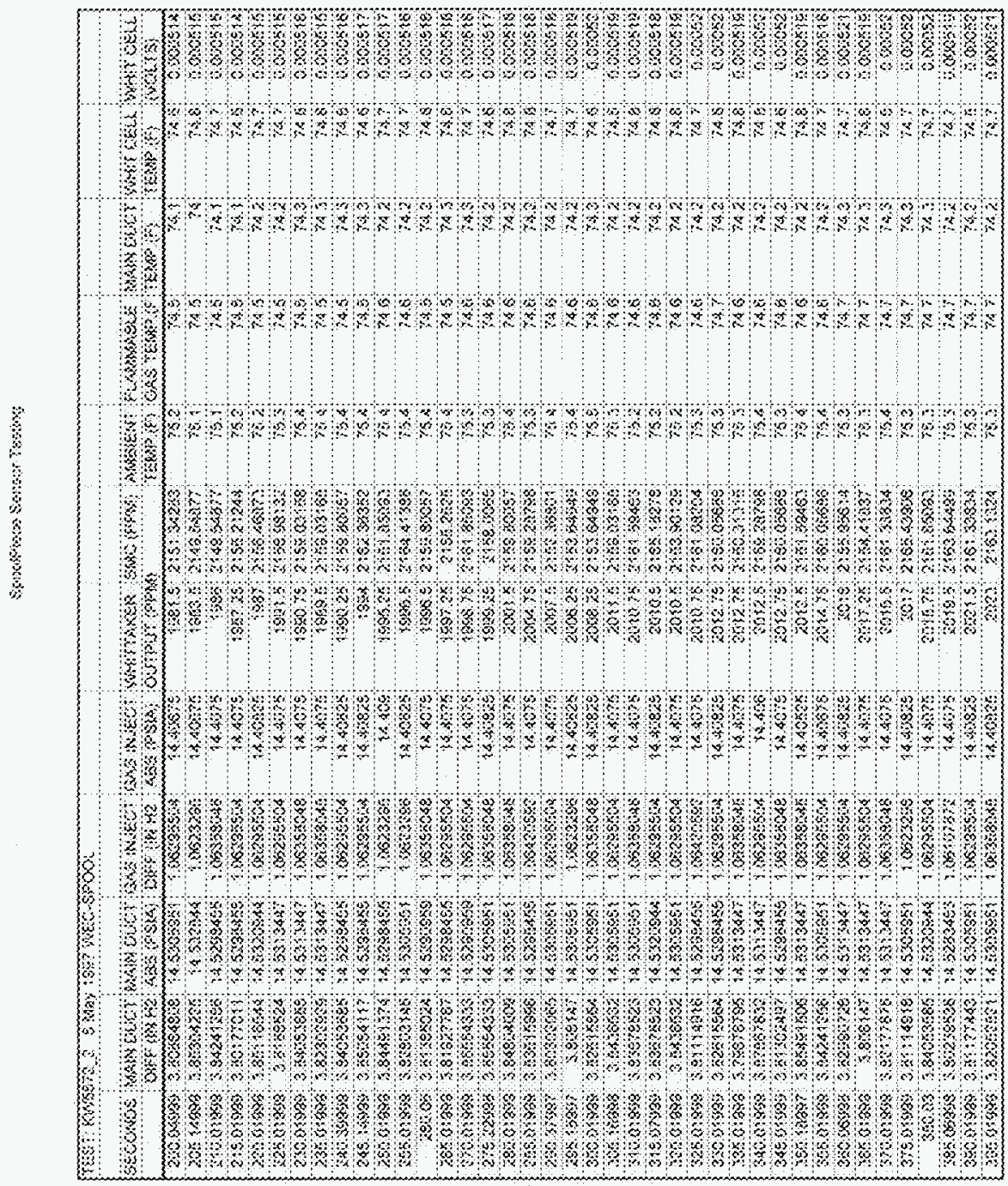




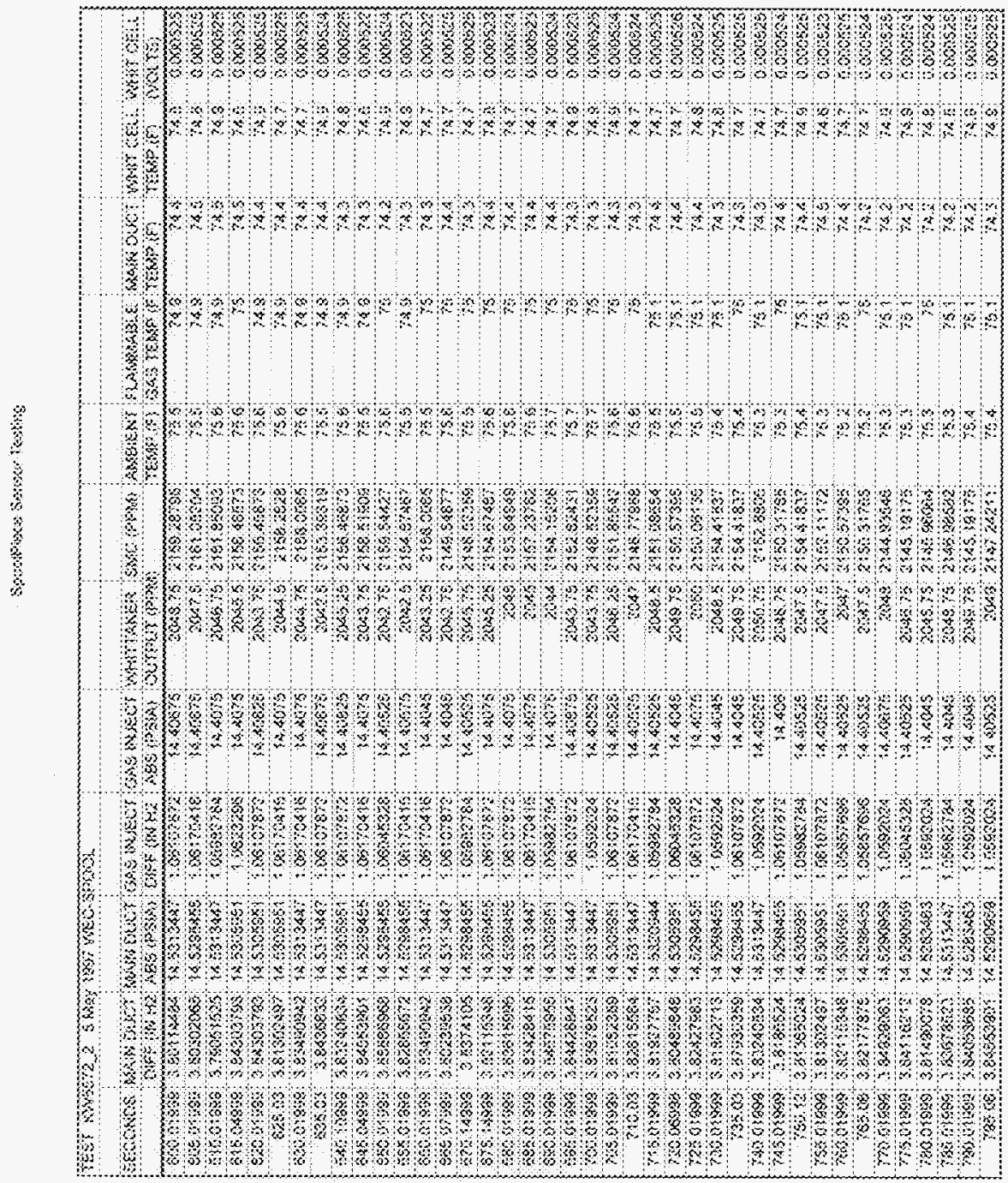




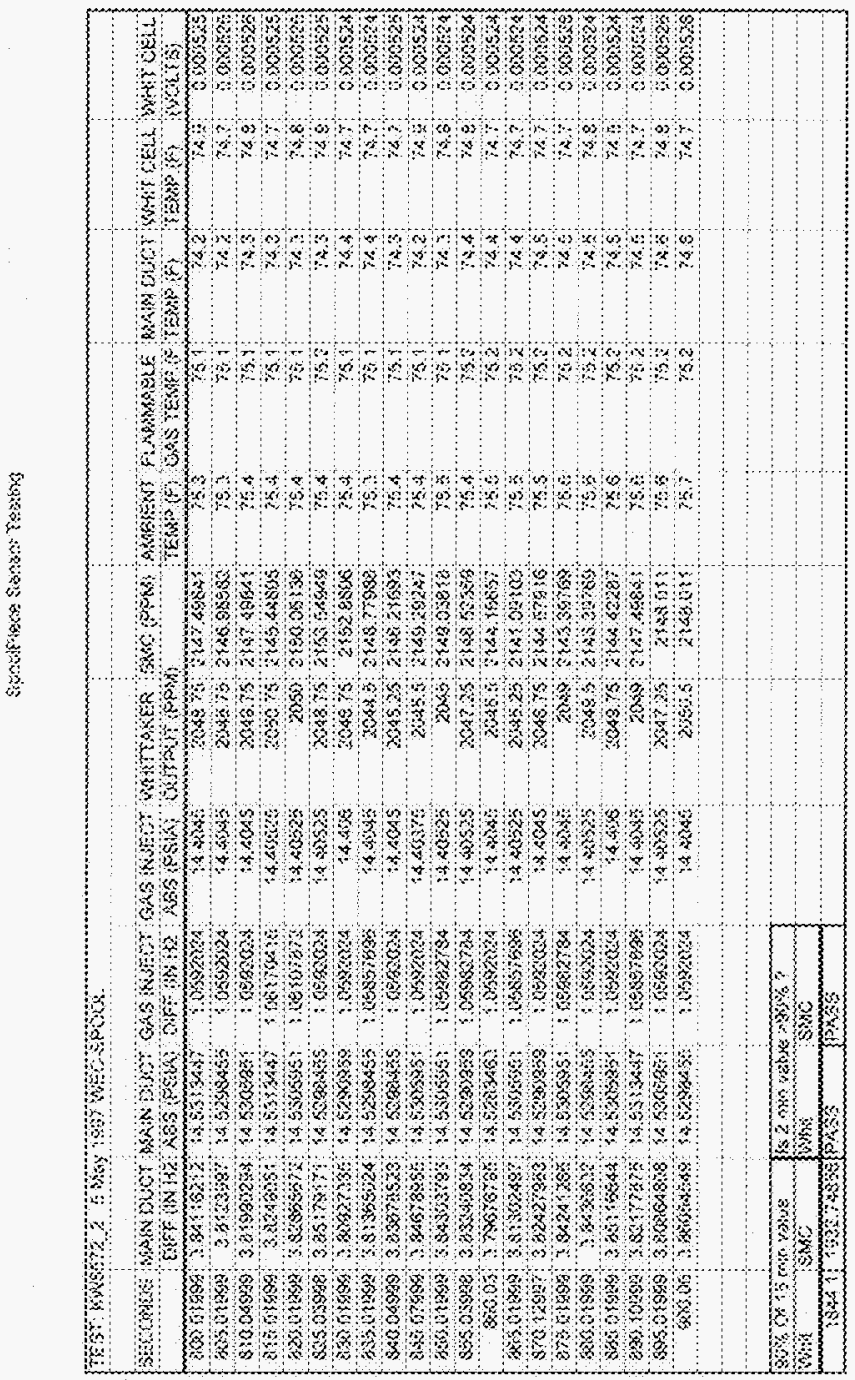




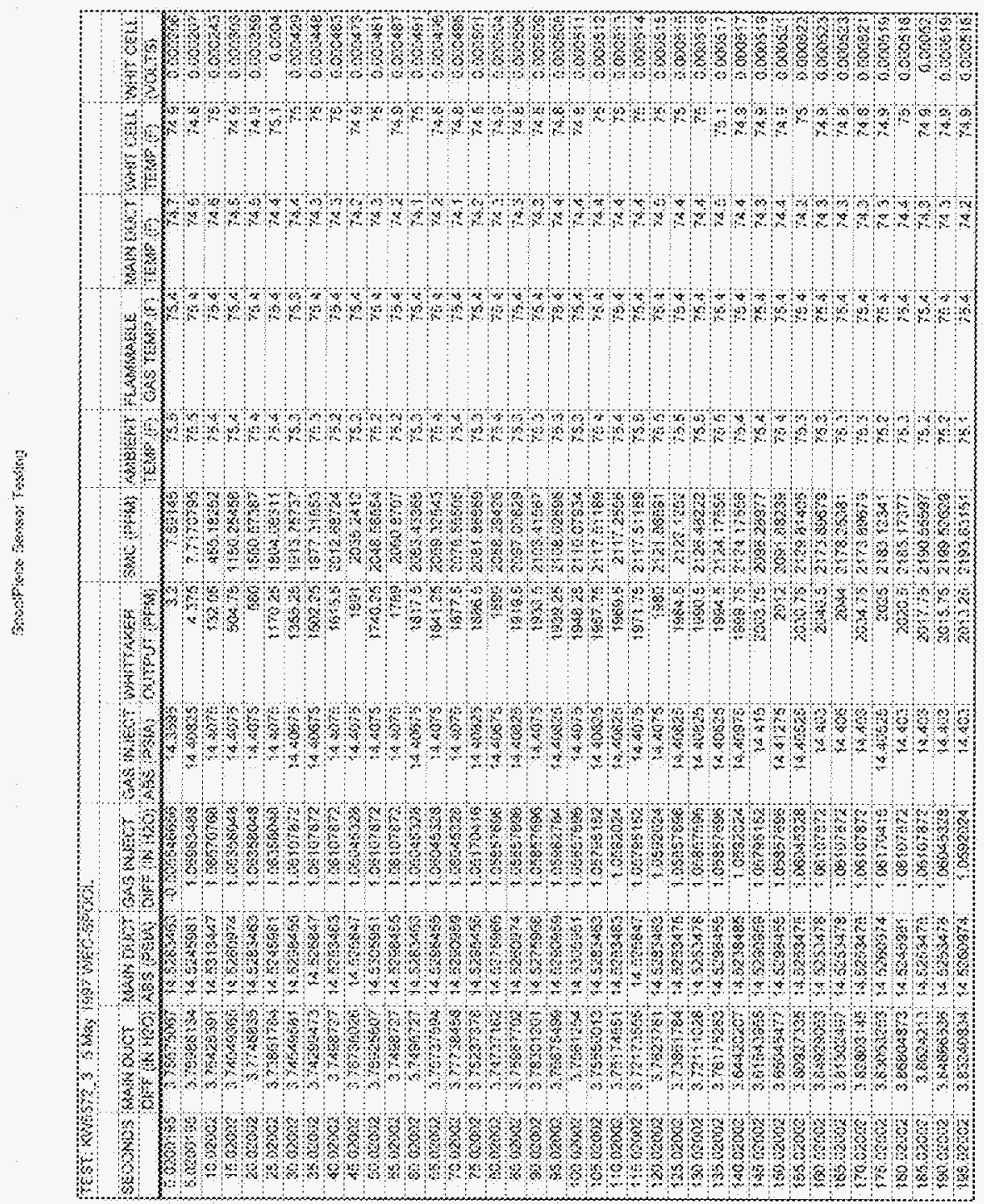




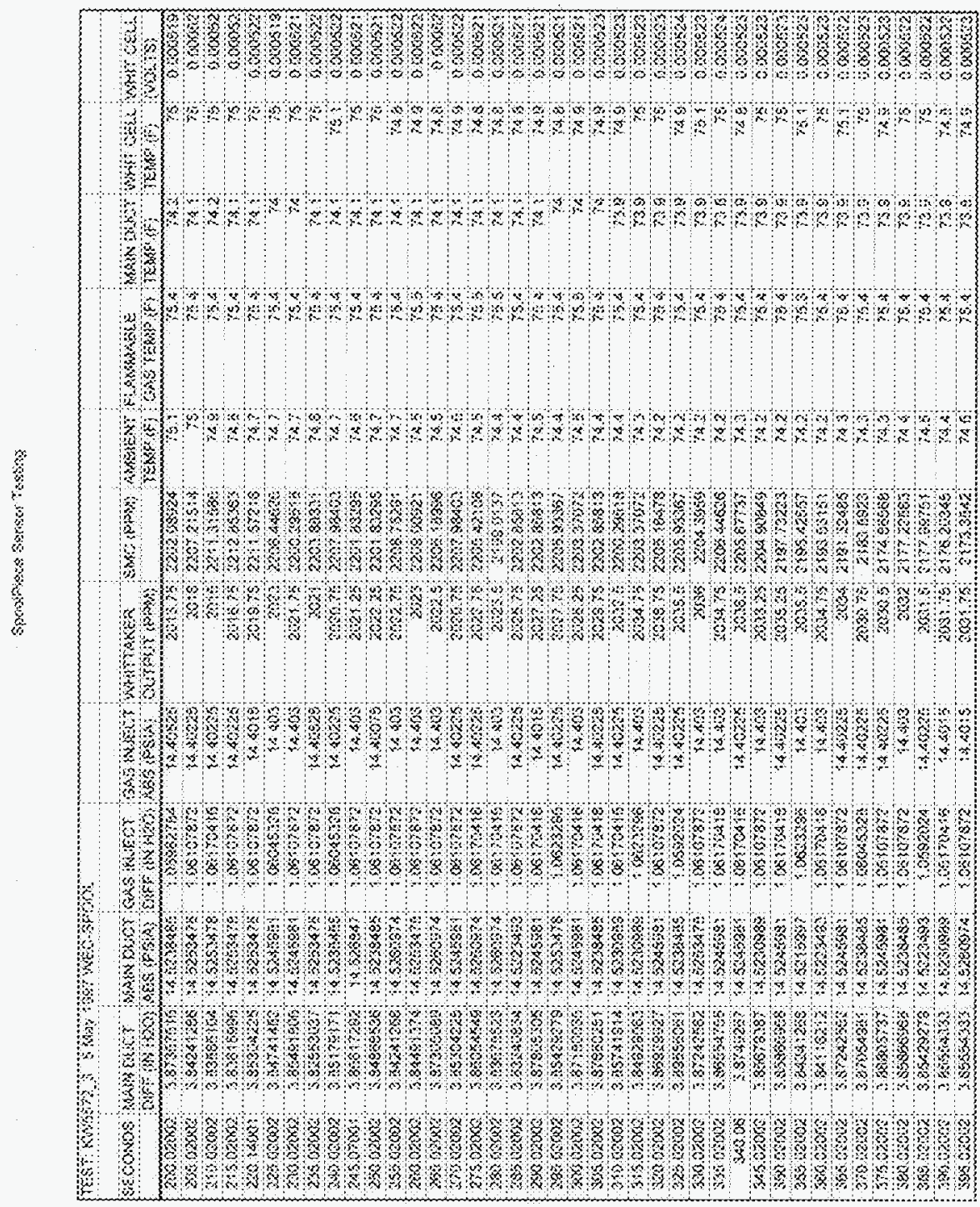




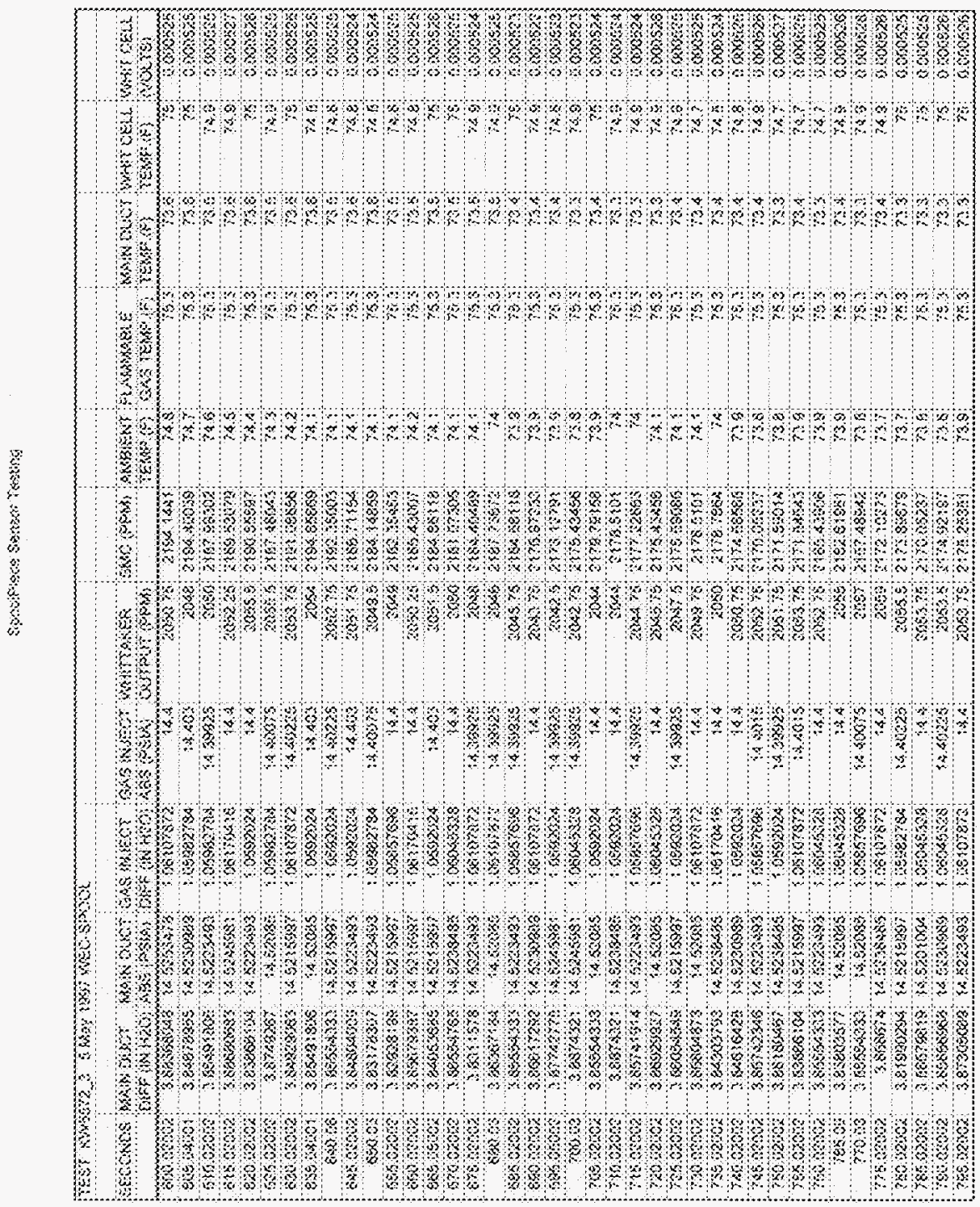




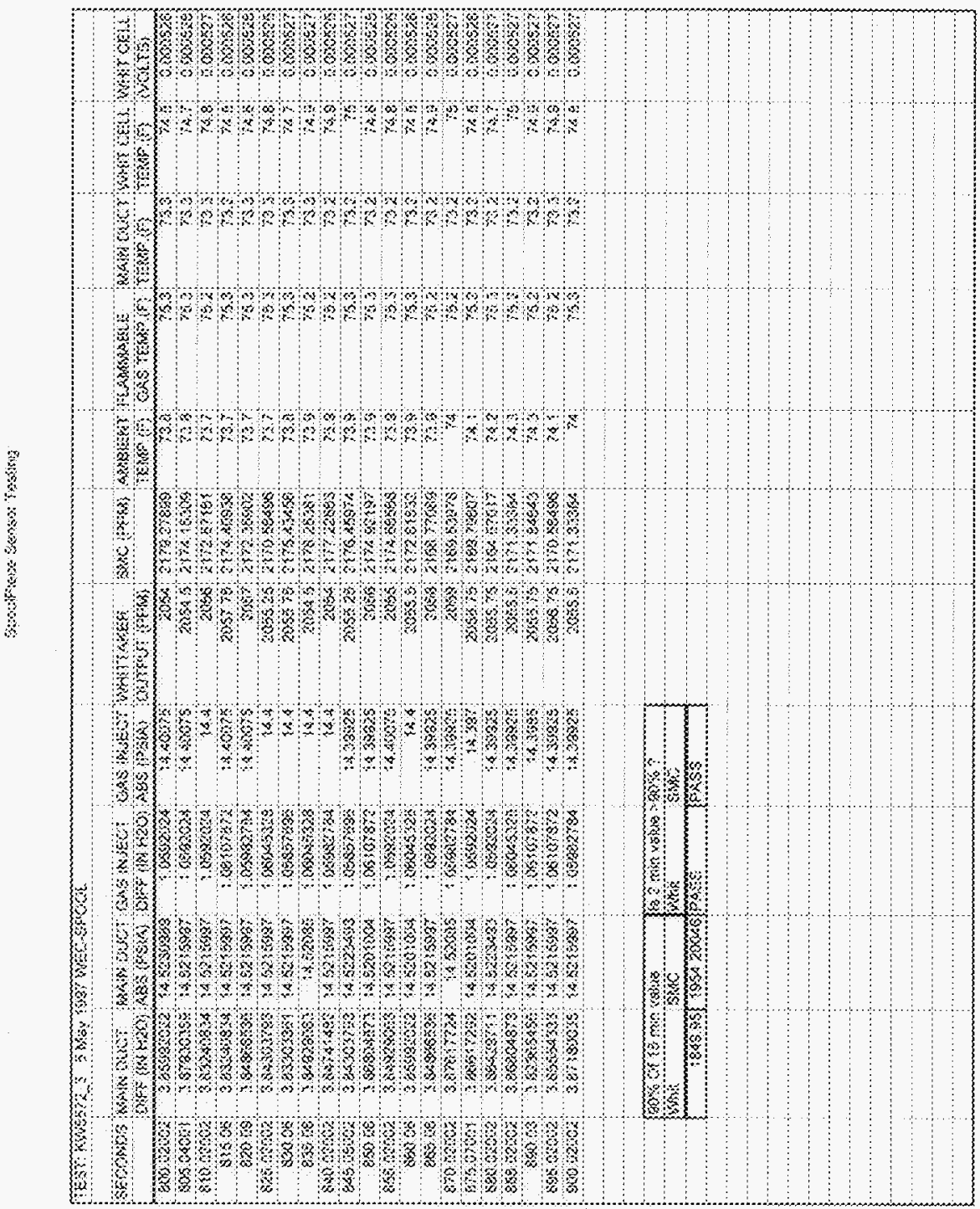




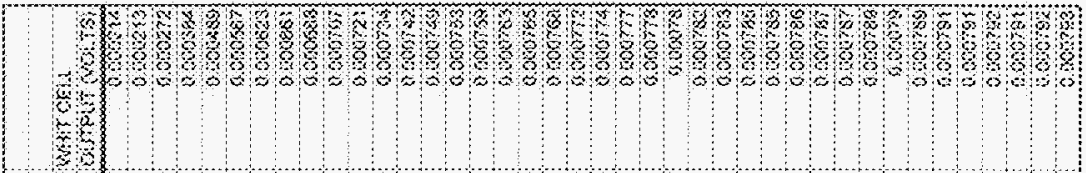

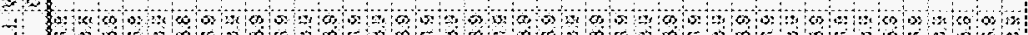

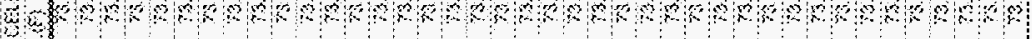
梦

tits

to

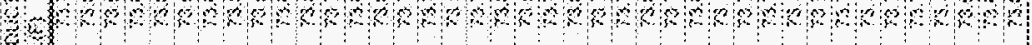
格

3

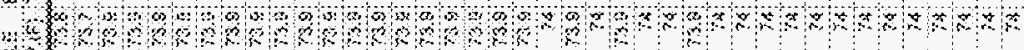
as

列

制

320 w

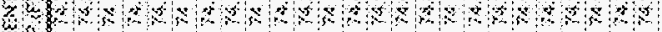

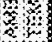

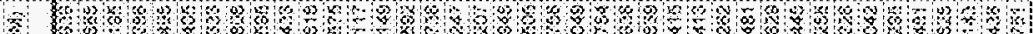

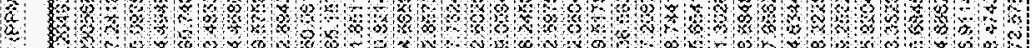

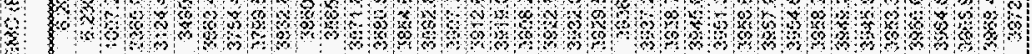

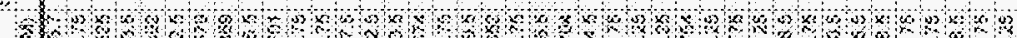

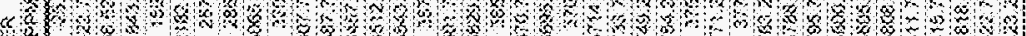
स.

.

\%:

\%

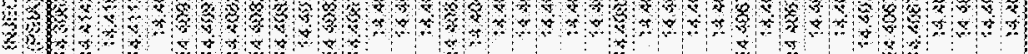

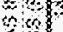

20 wom

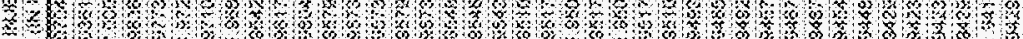

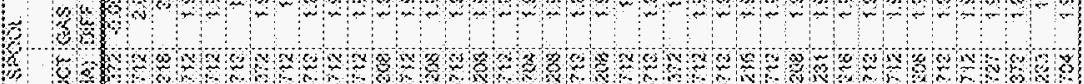

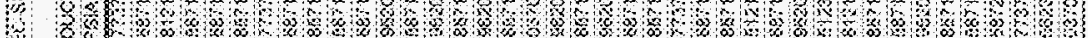

3 W

a

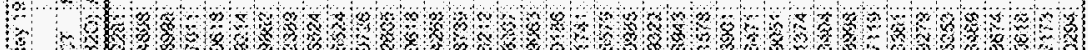

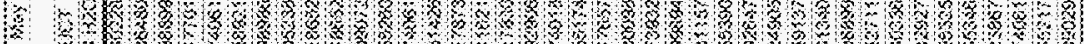

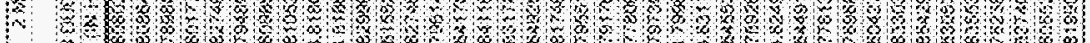

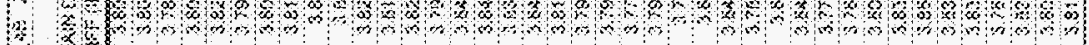

w ${ }^{2}$ op

1 \%

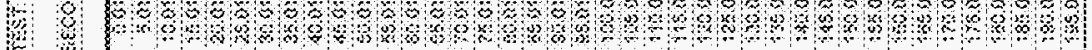




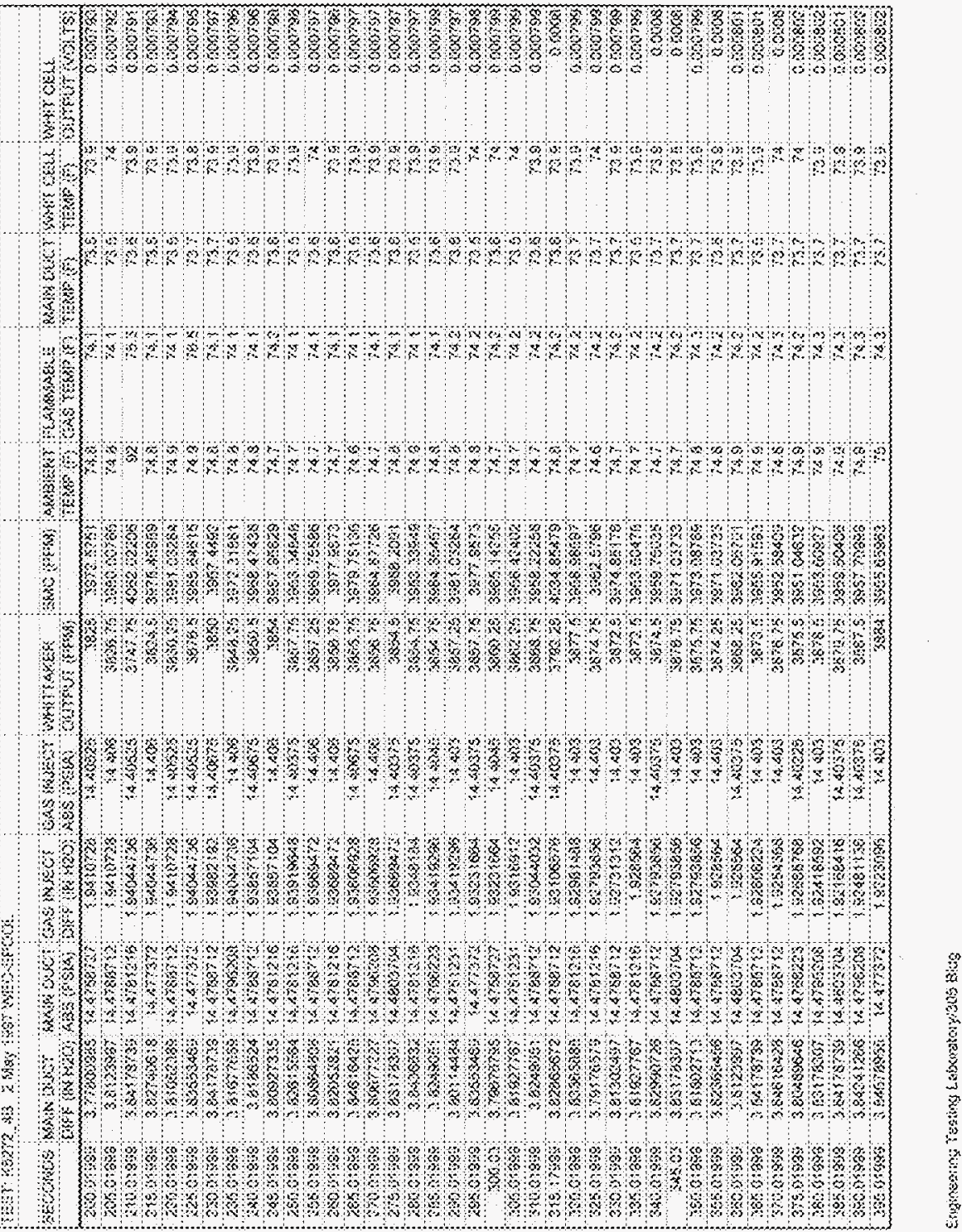




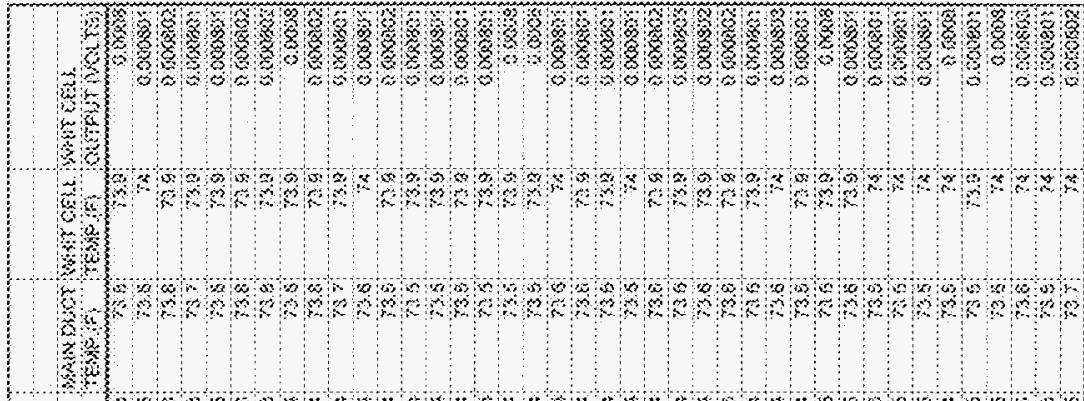
wa 紊

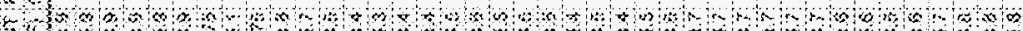
से 茨

- \%

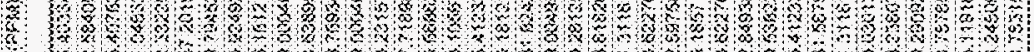

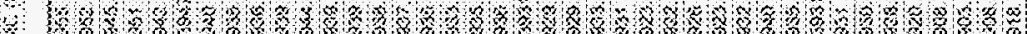

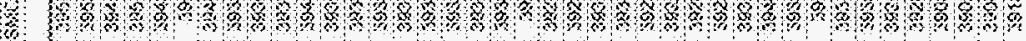

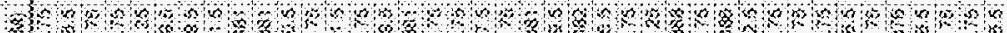

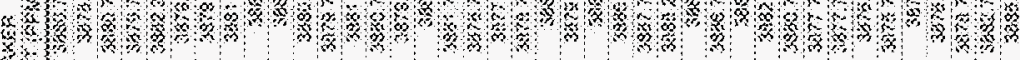

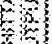

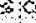

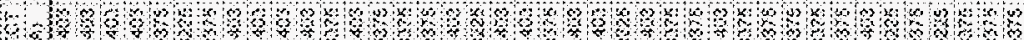
बa

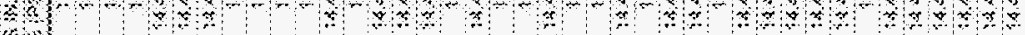
s.

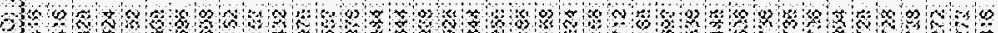

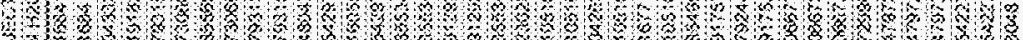
$2 \mathrm{~d}$,

(1)

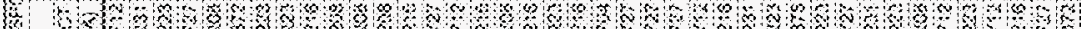

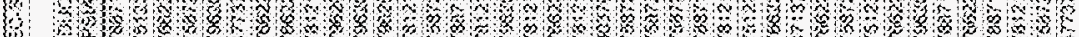

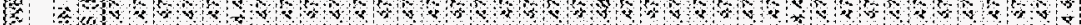

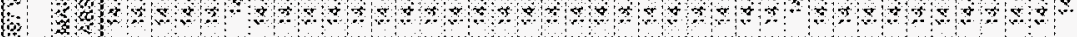

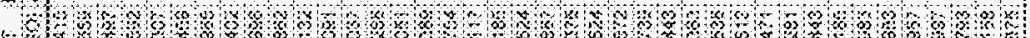

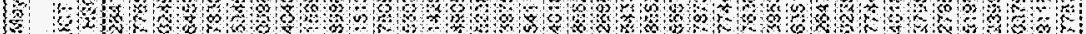

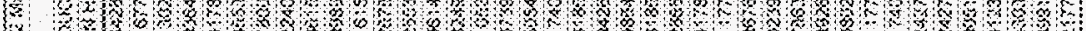

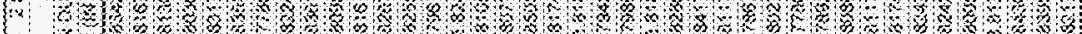

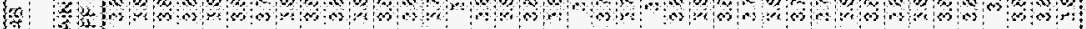

or:

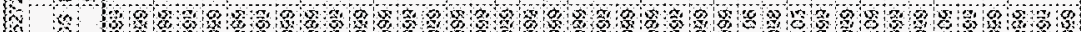

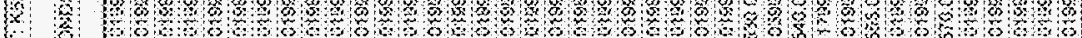
W 


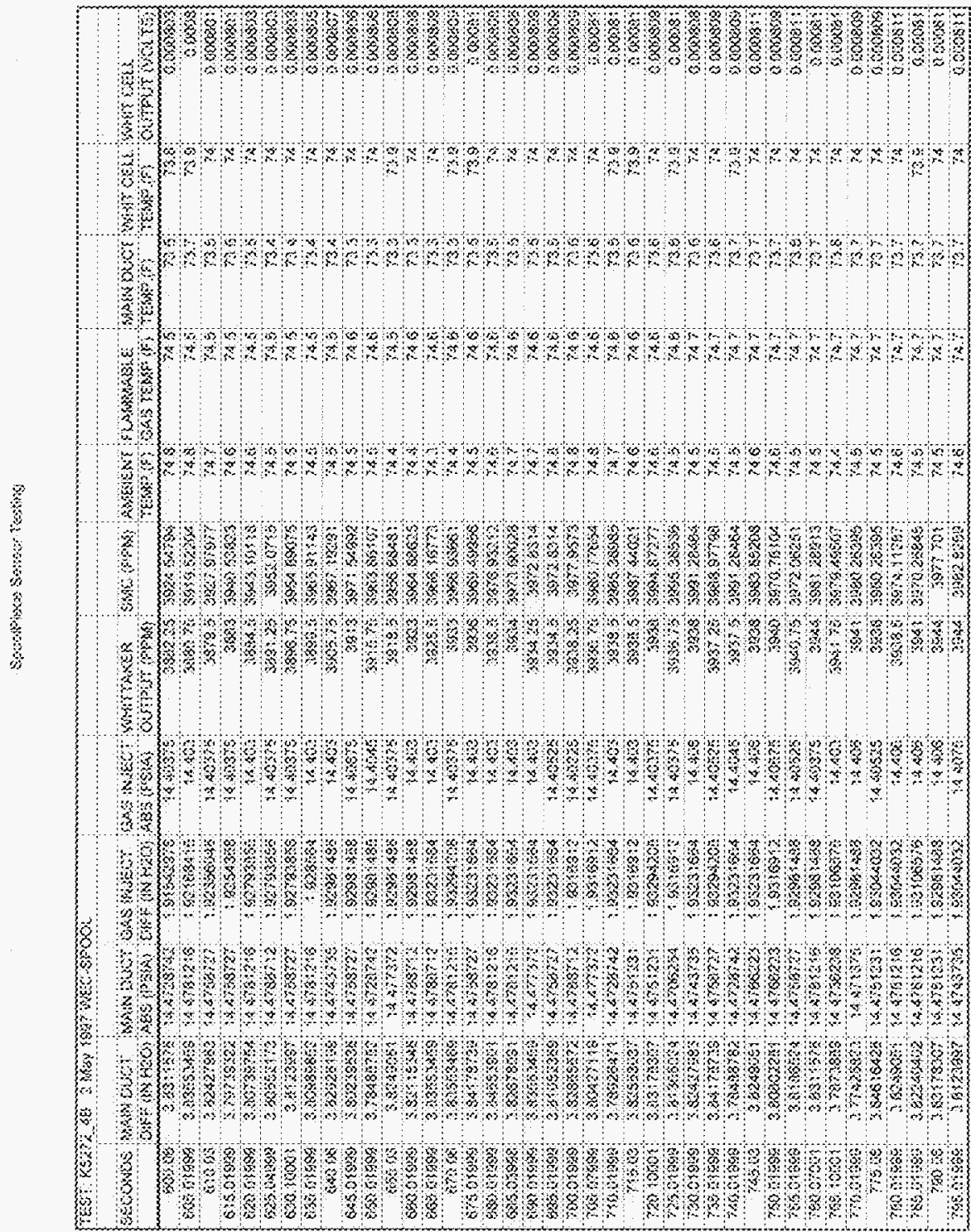




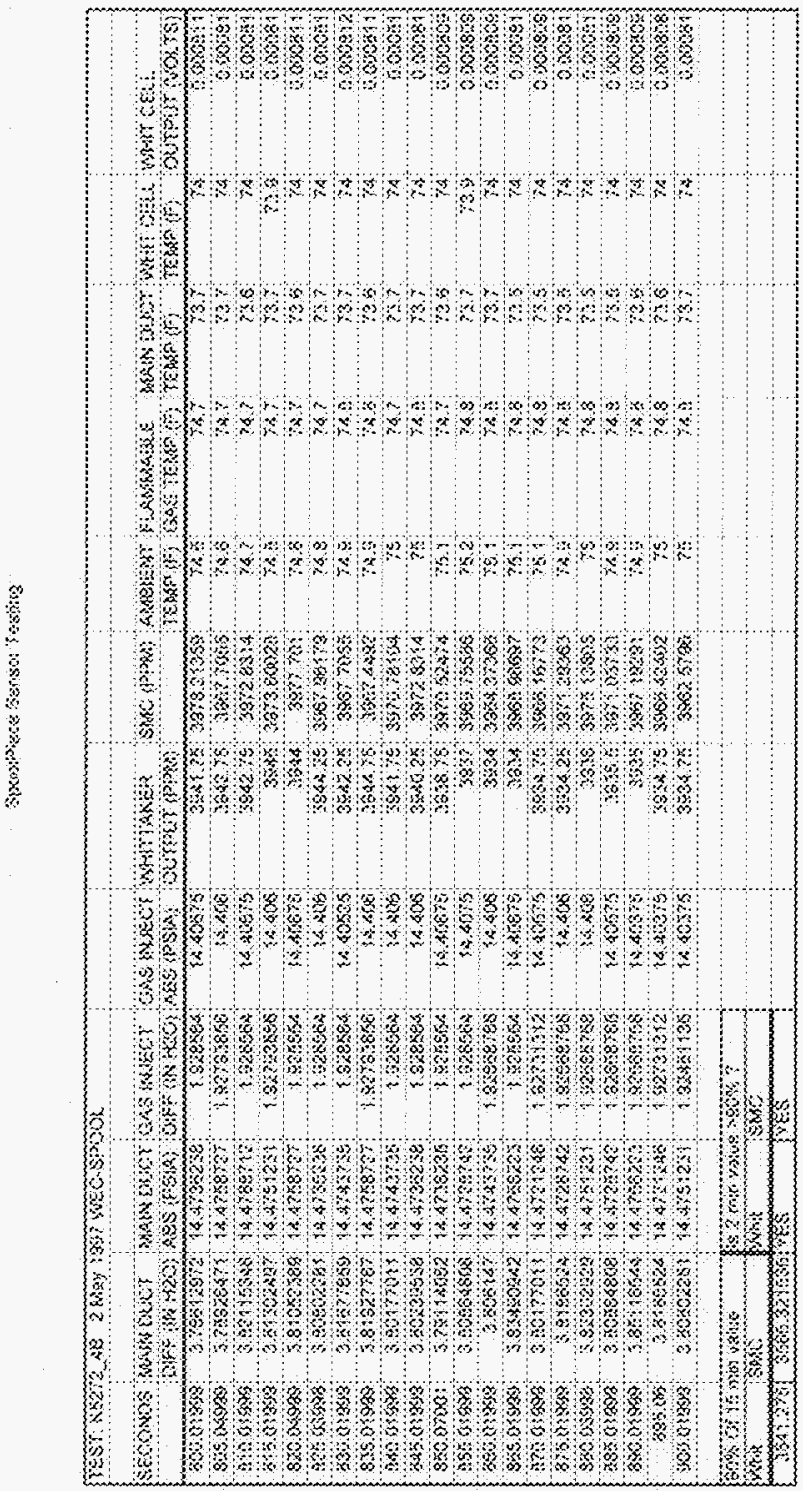




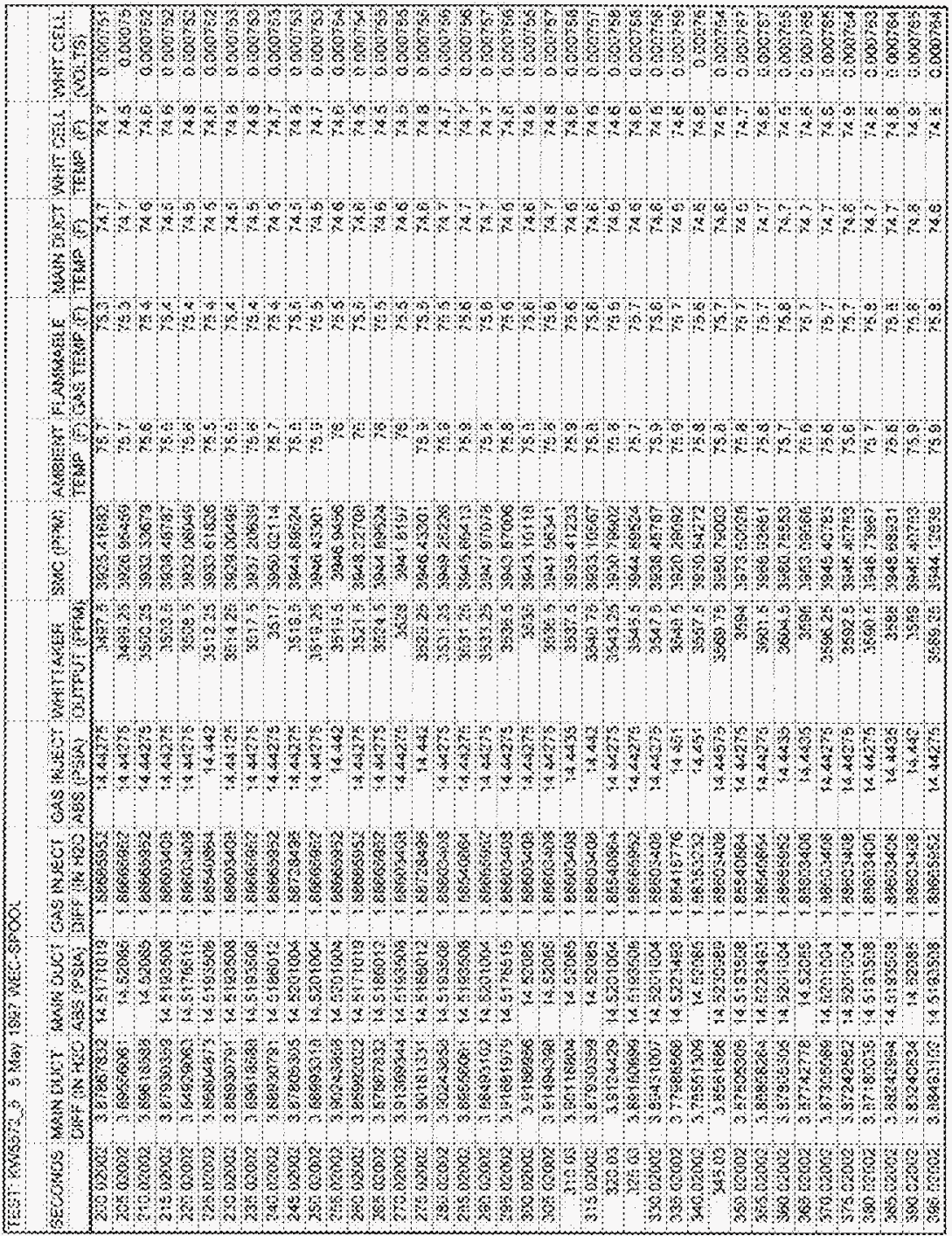




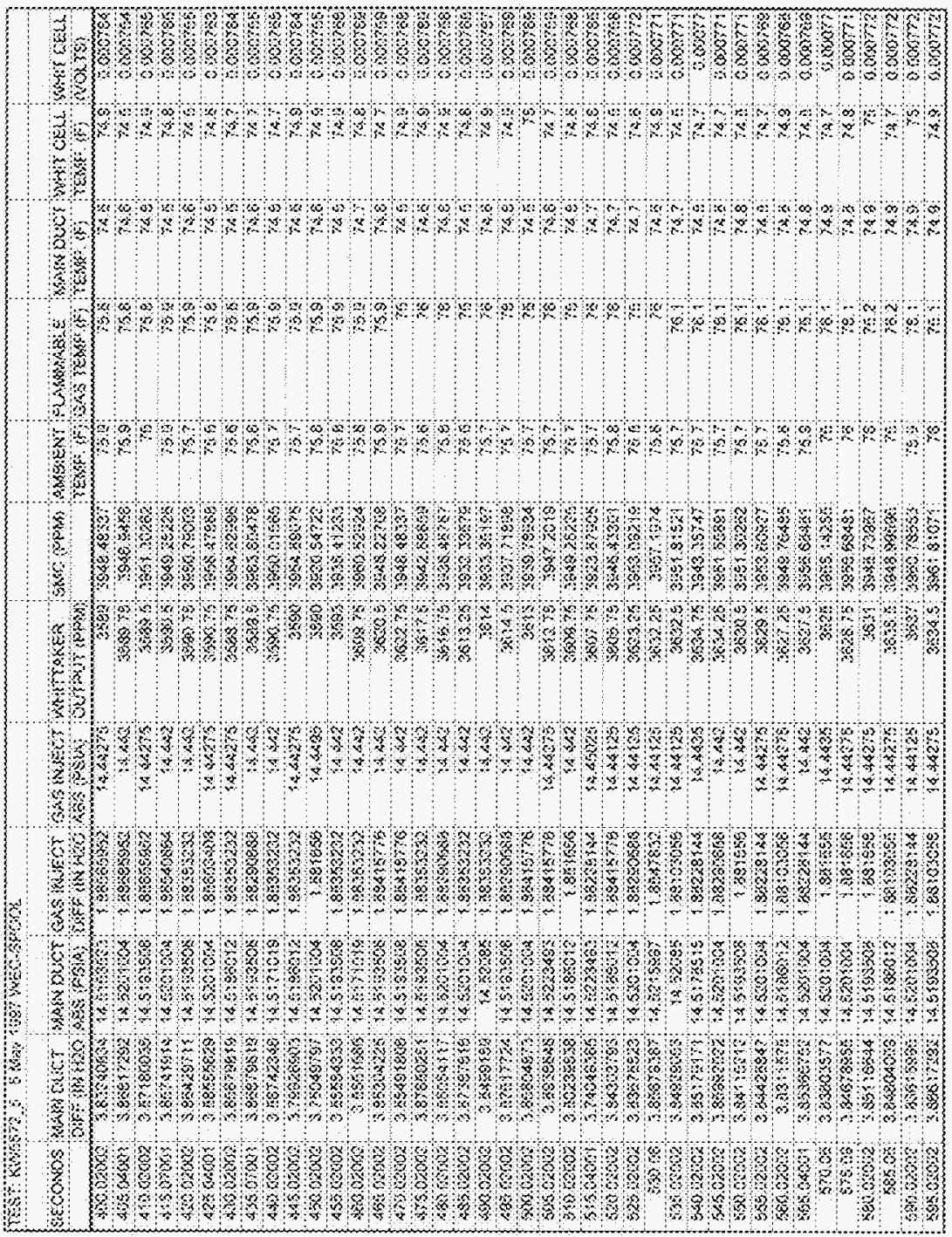




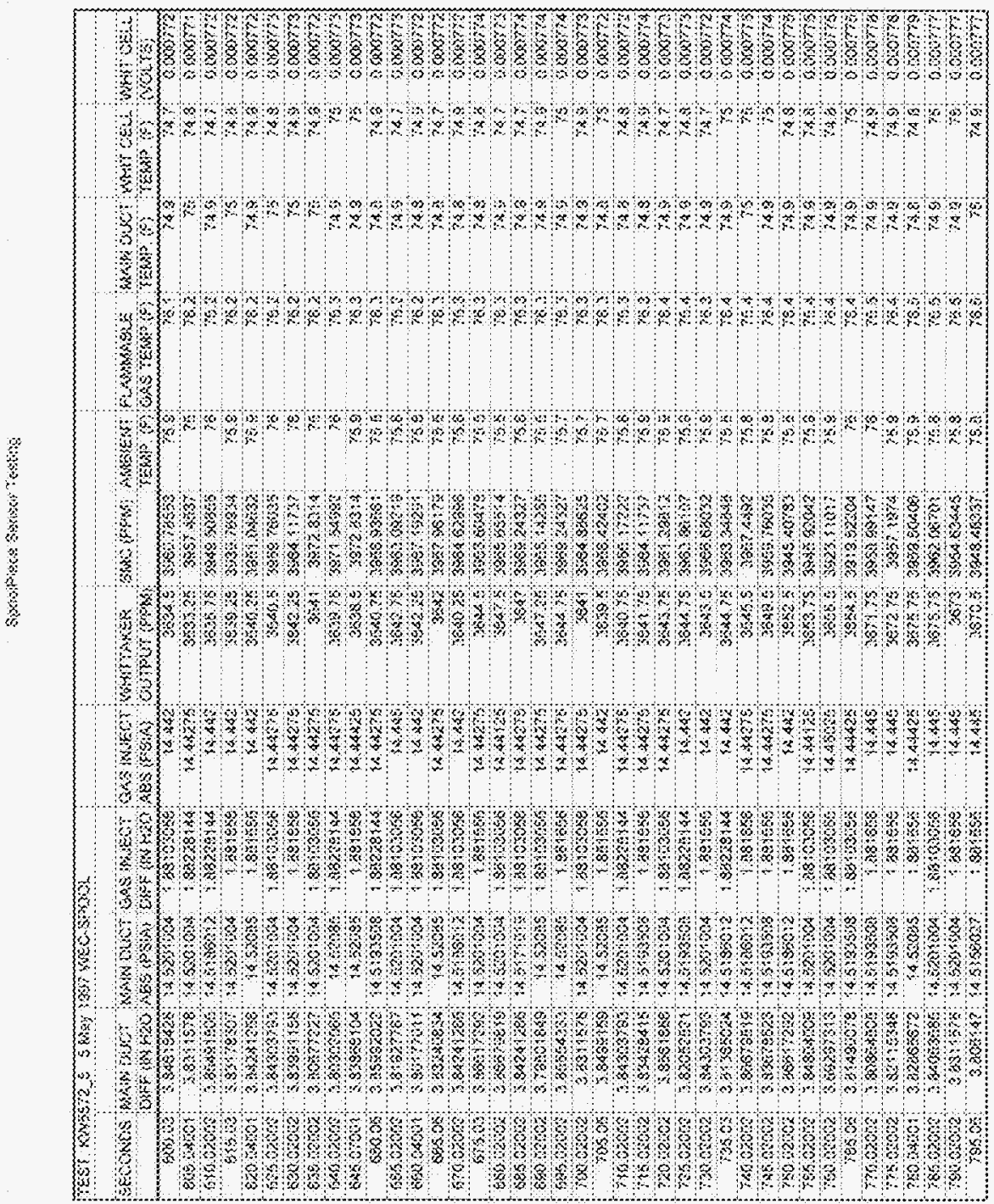




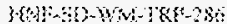

5x:? ?

iate !4?

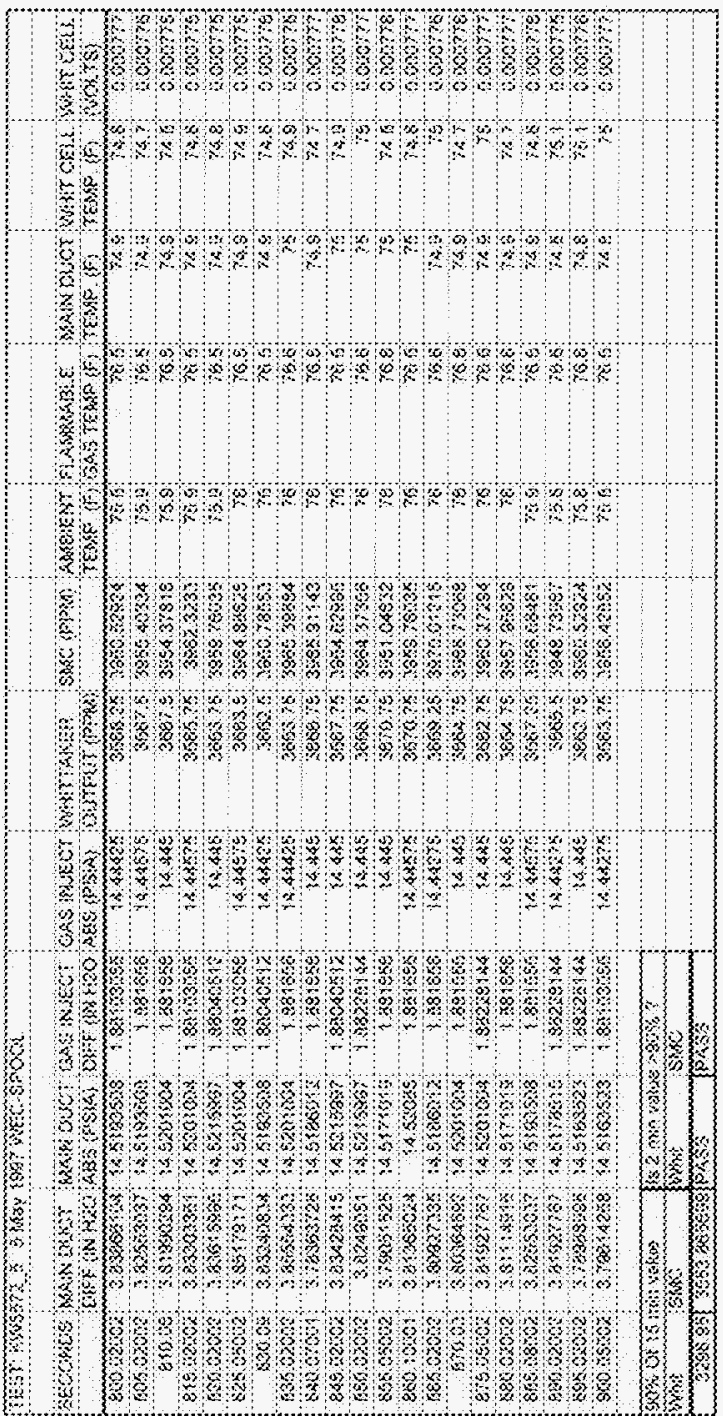




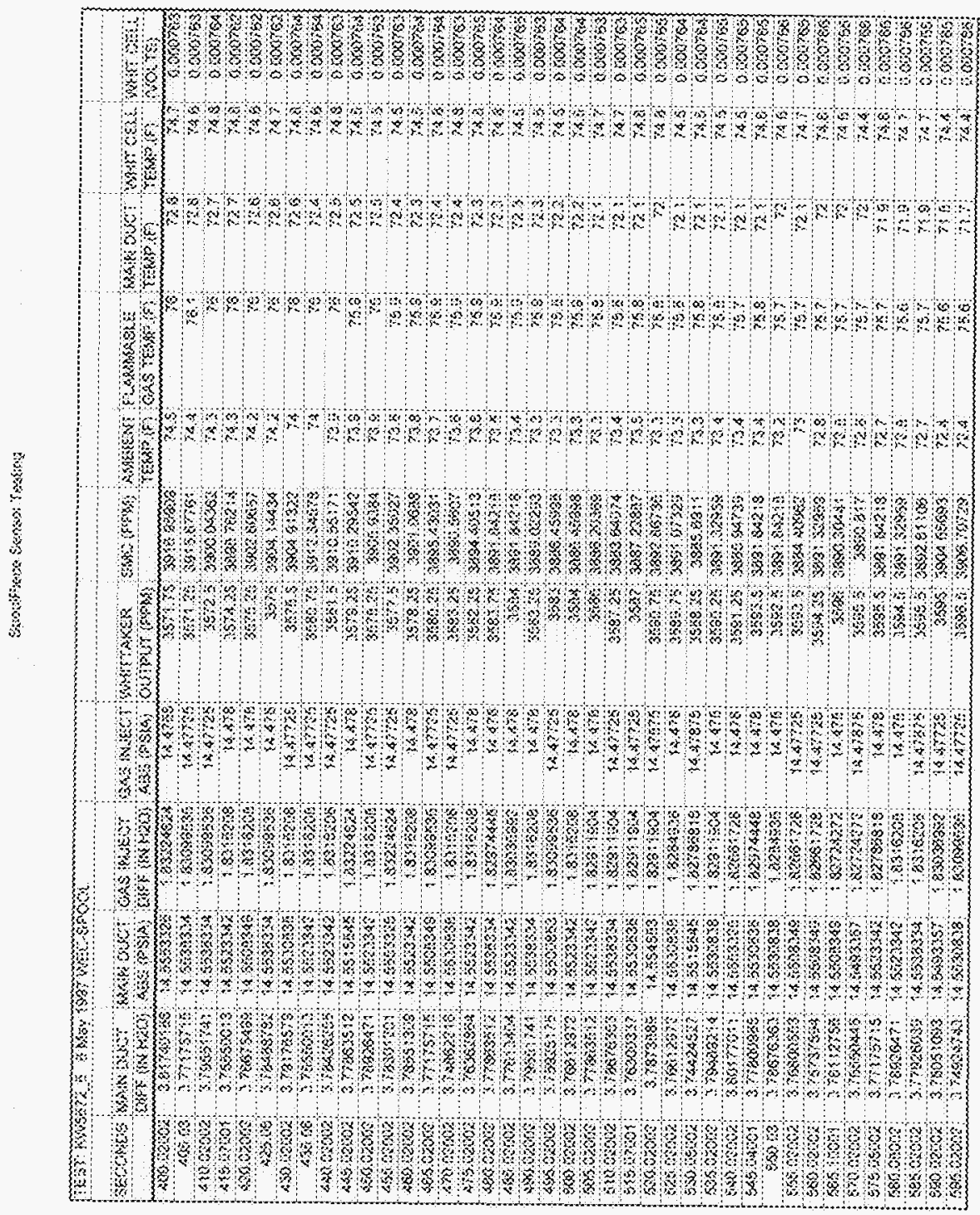




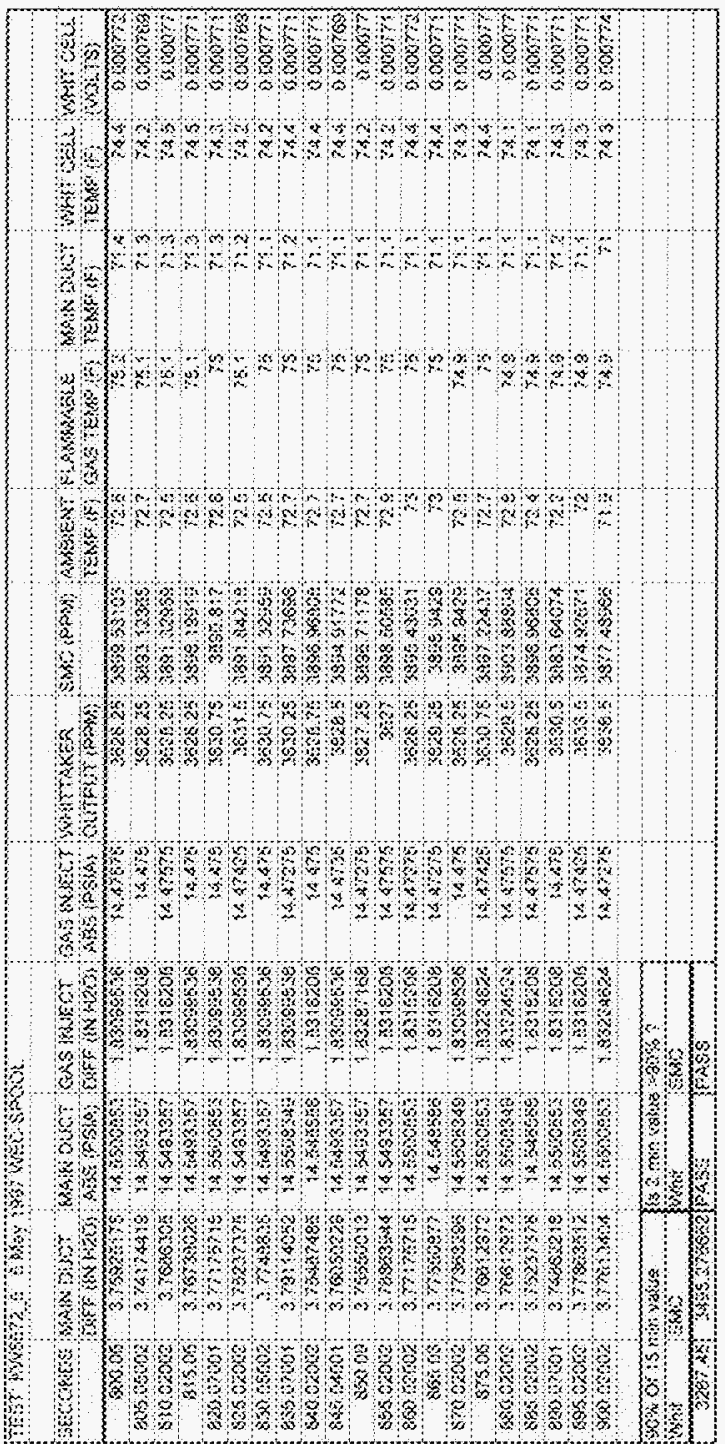




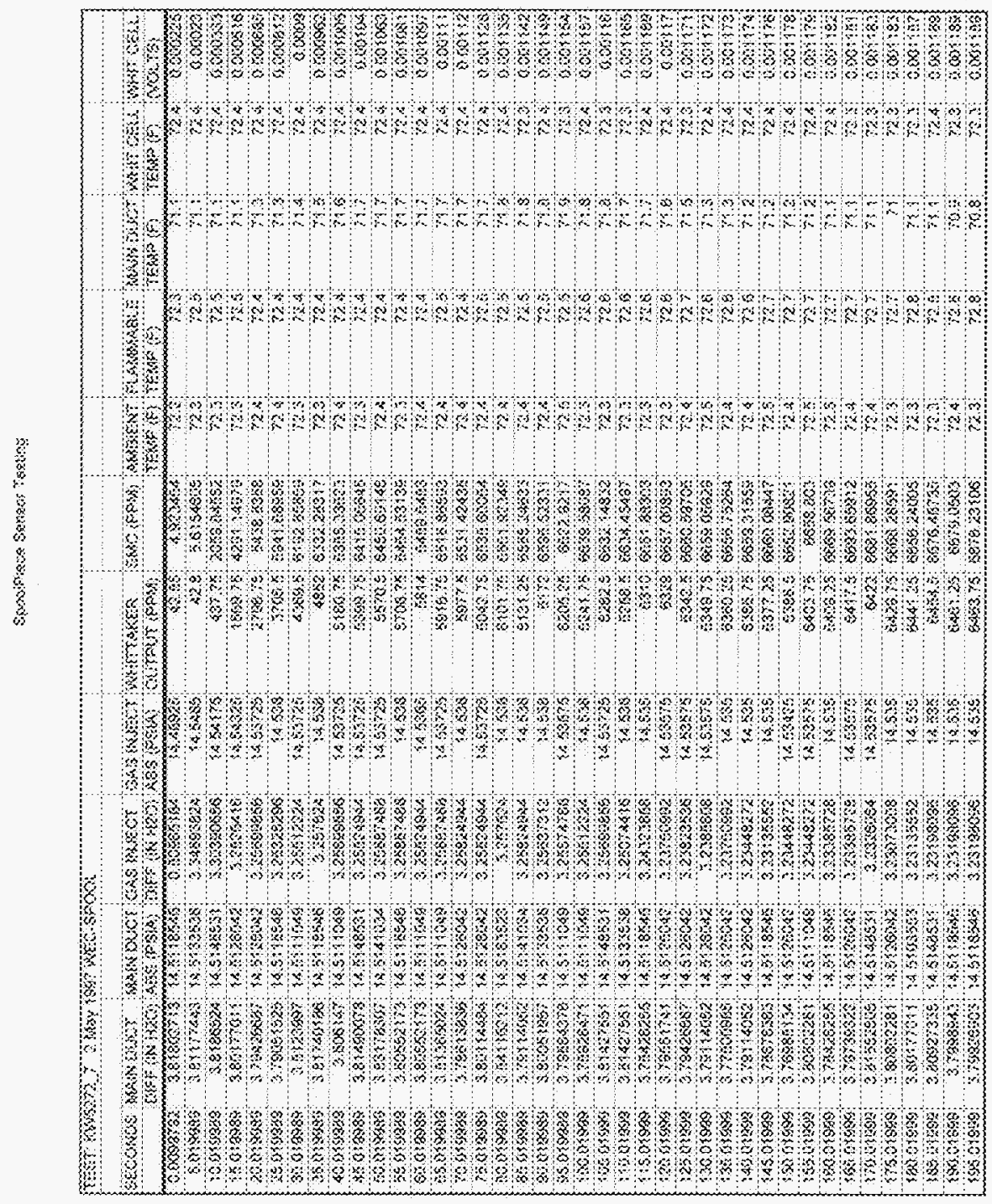




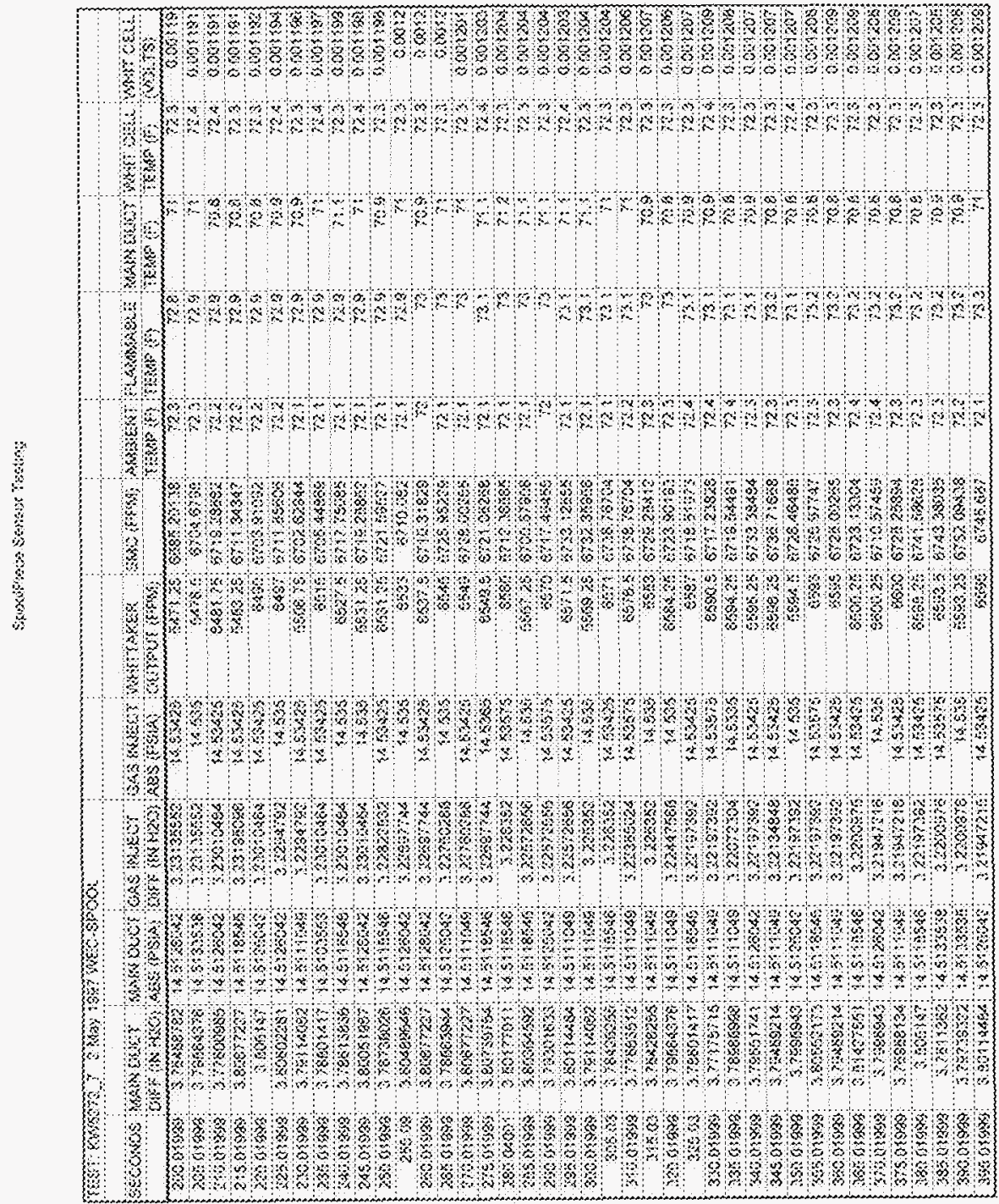




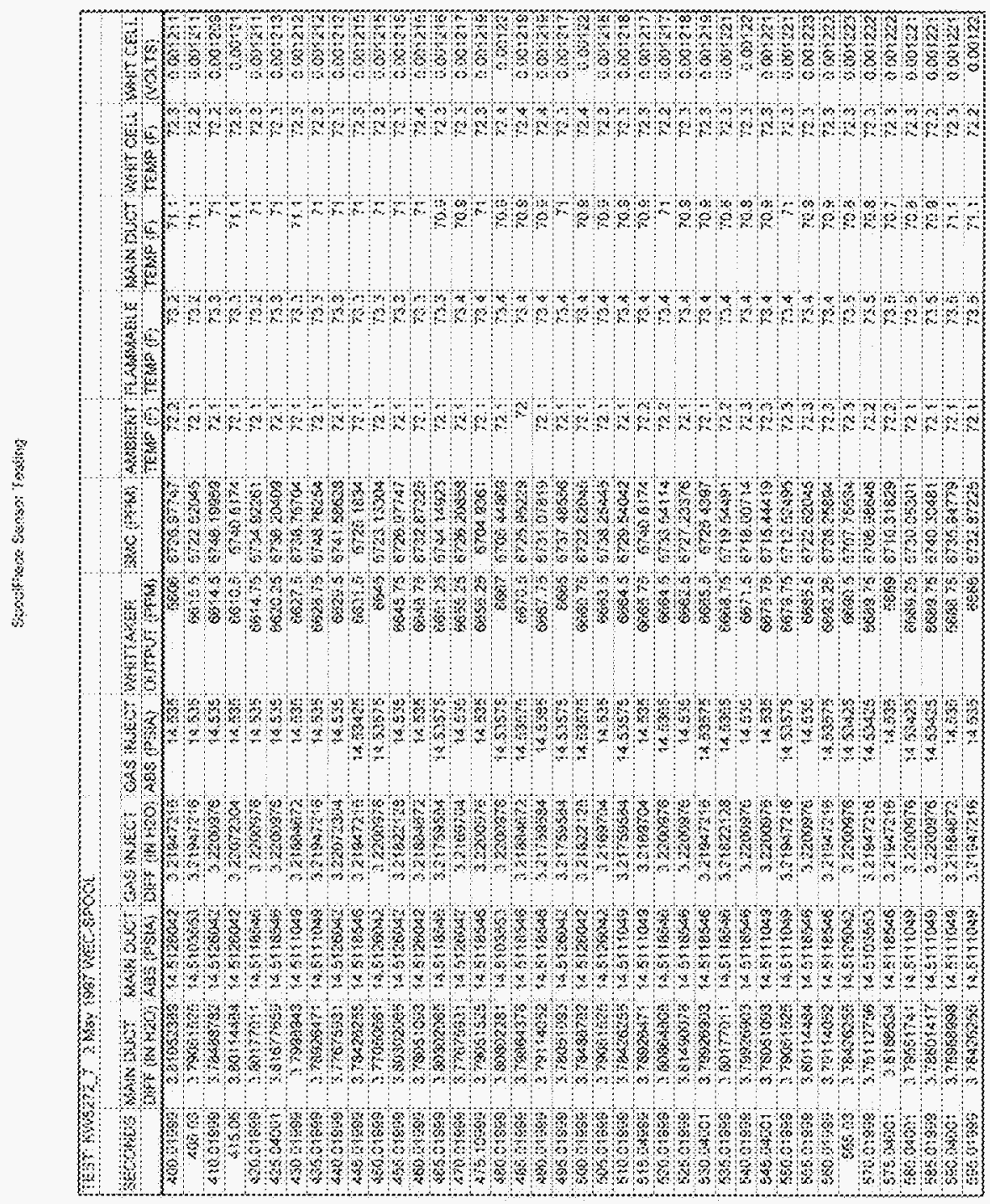




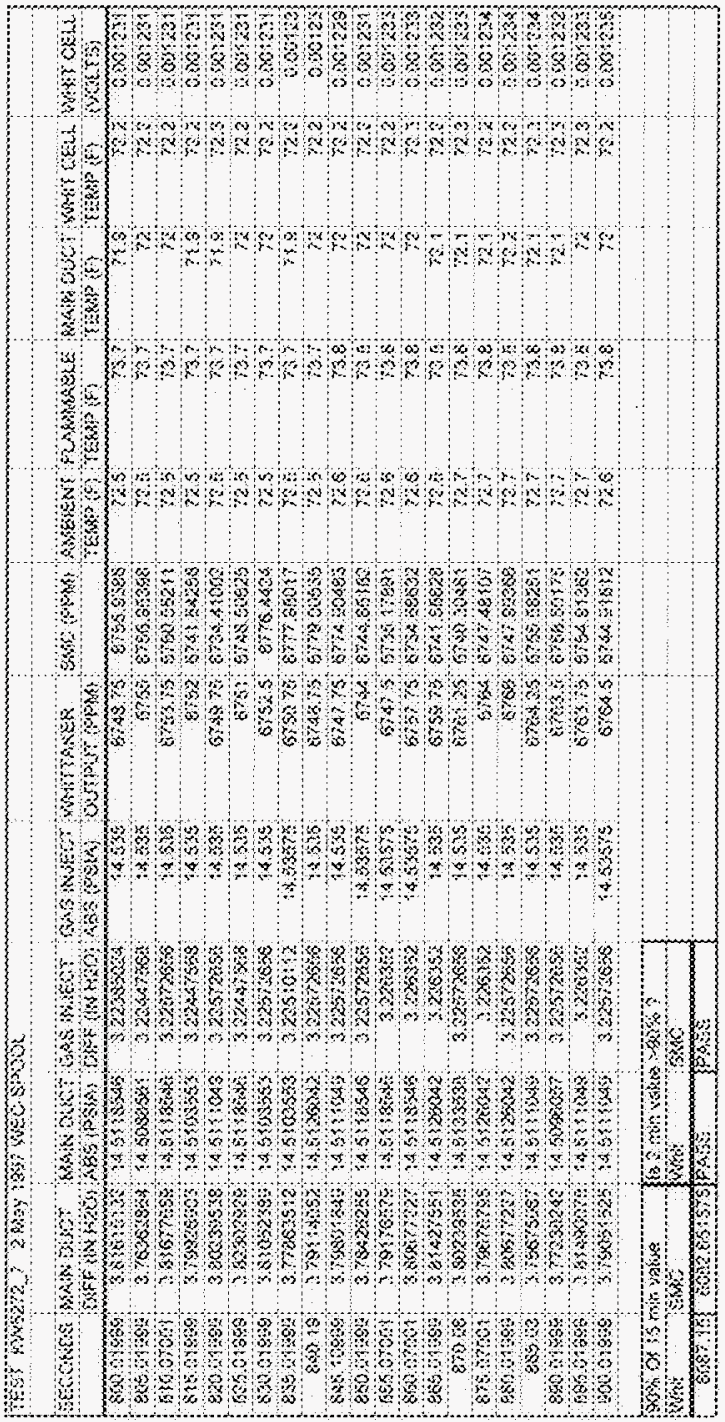




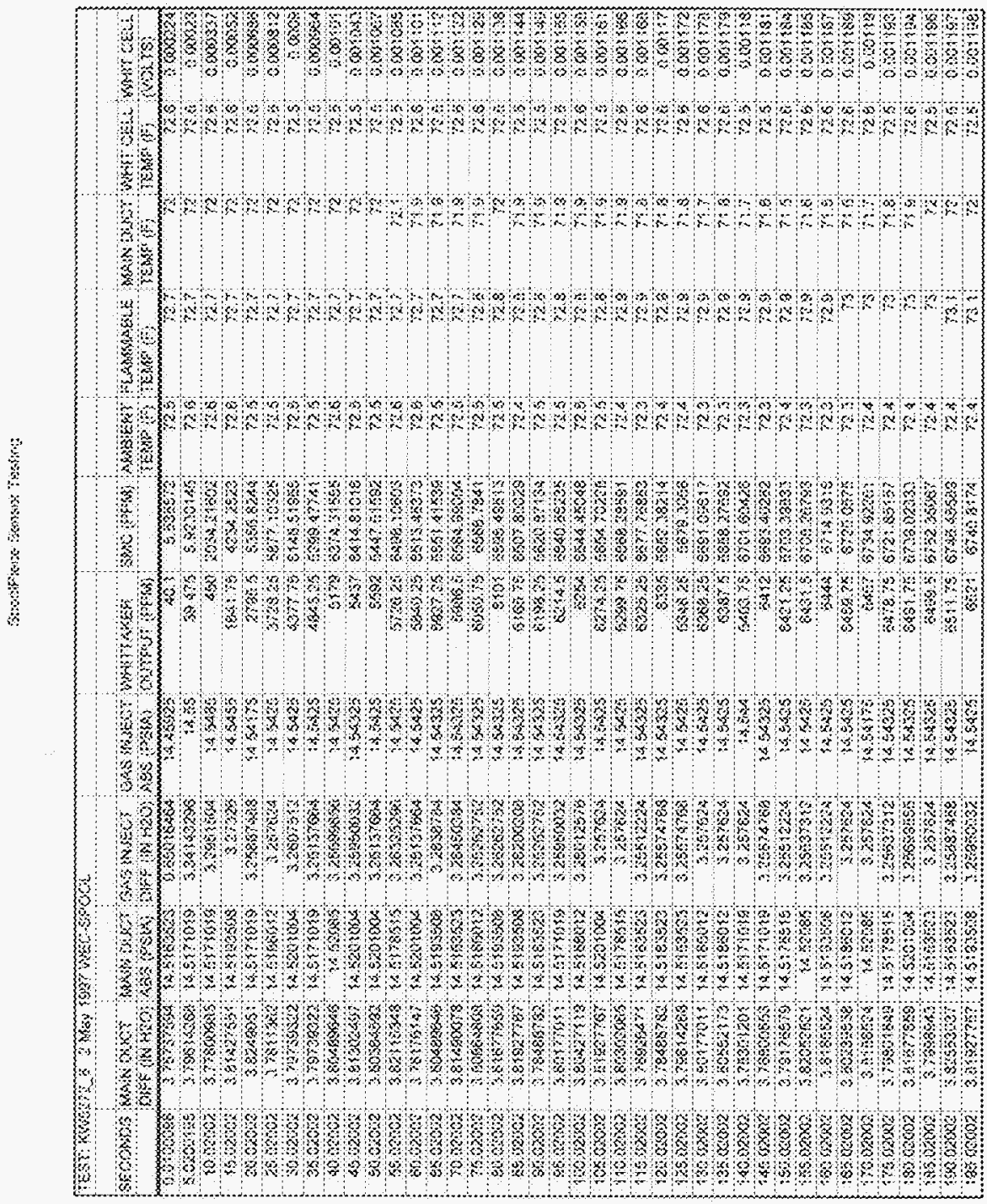




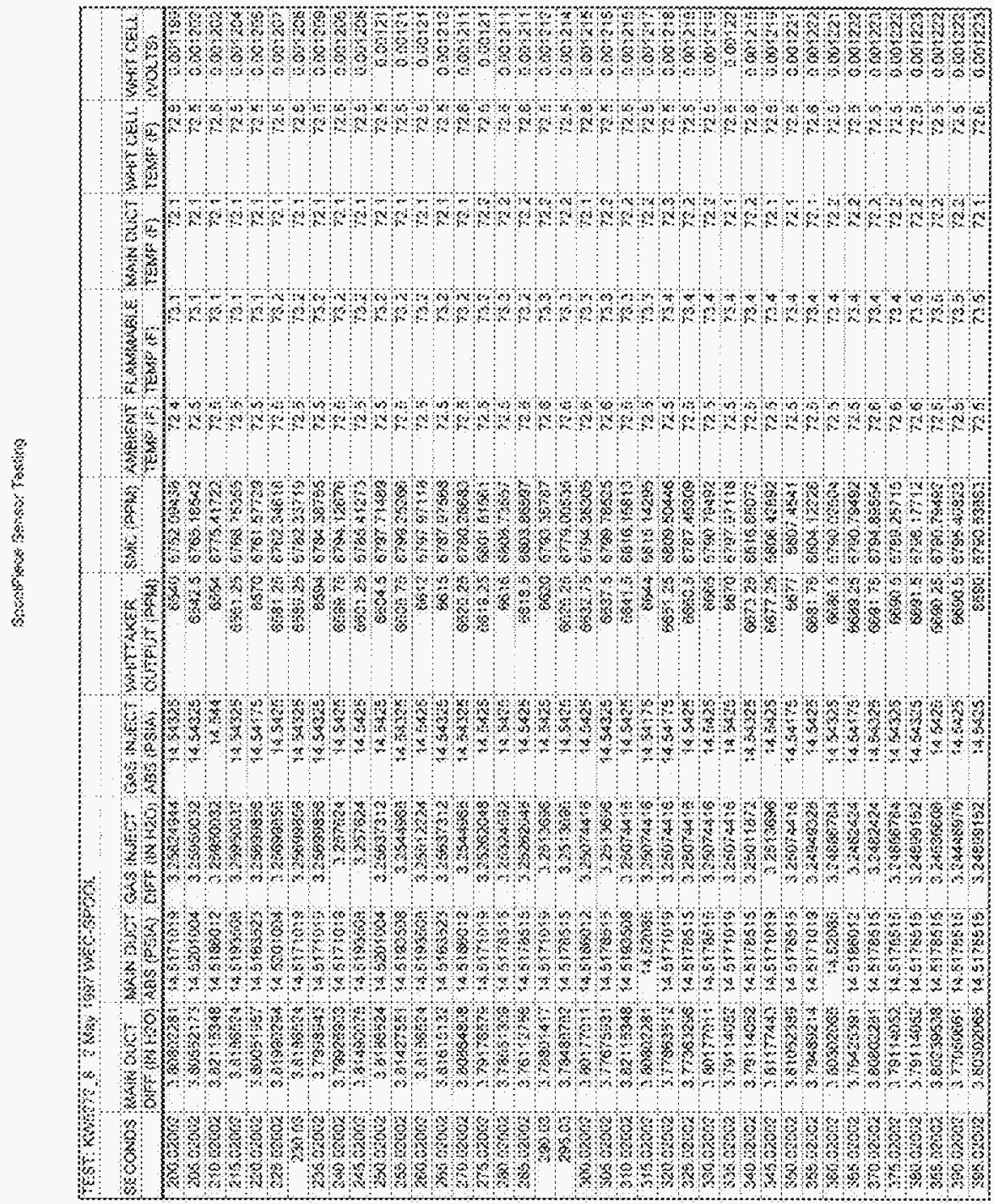


BNE. HOW

\{ter. 0

?as

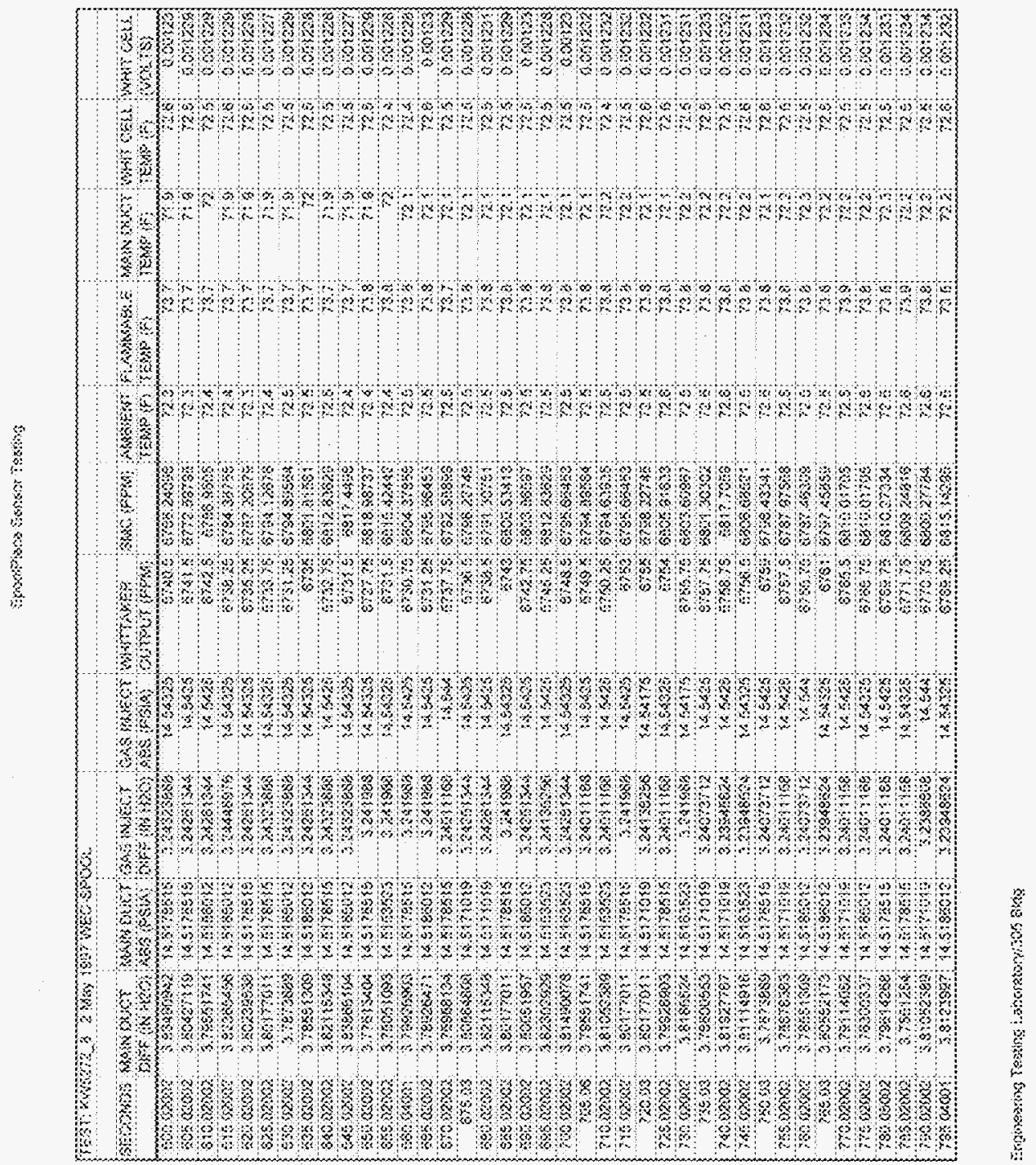




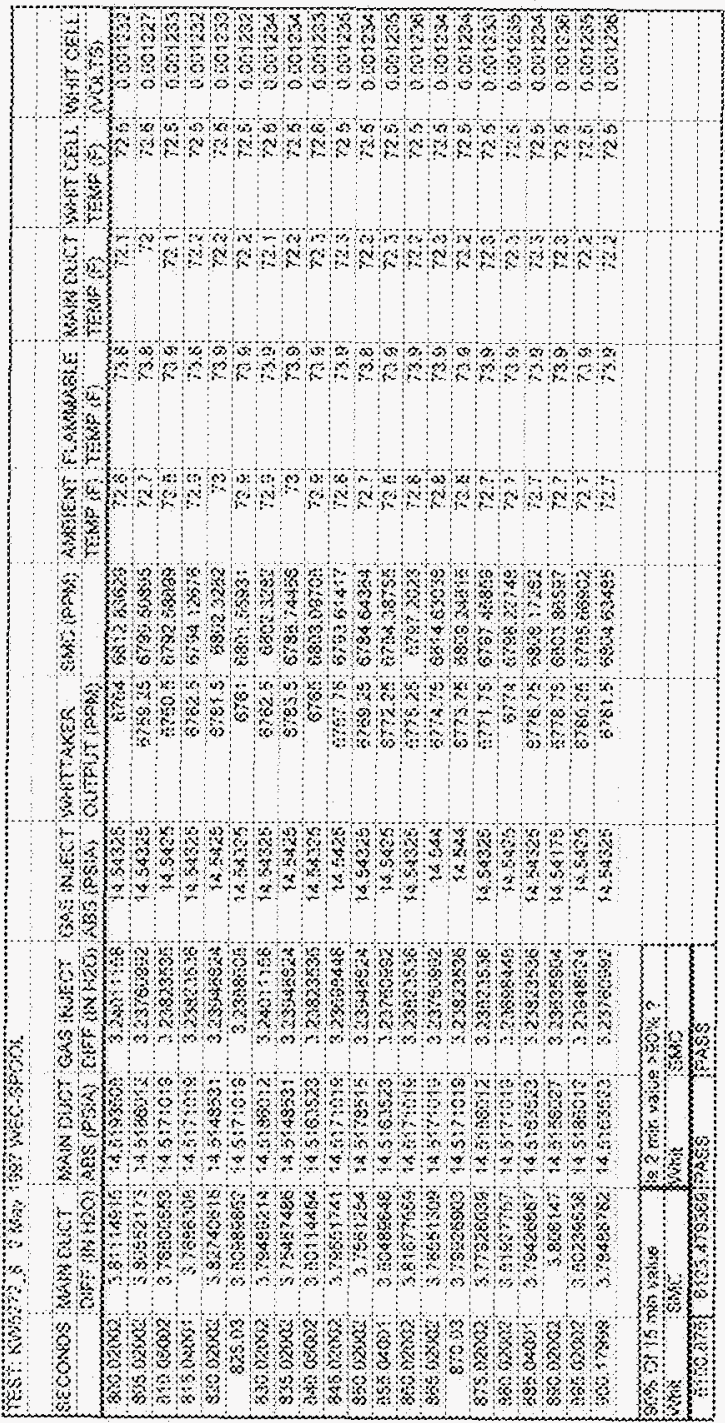




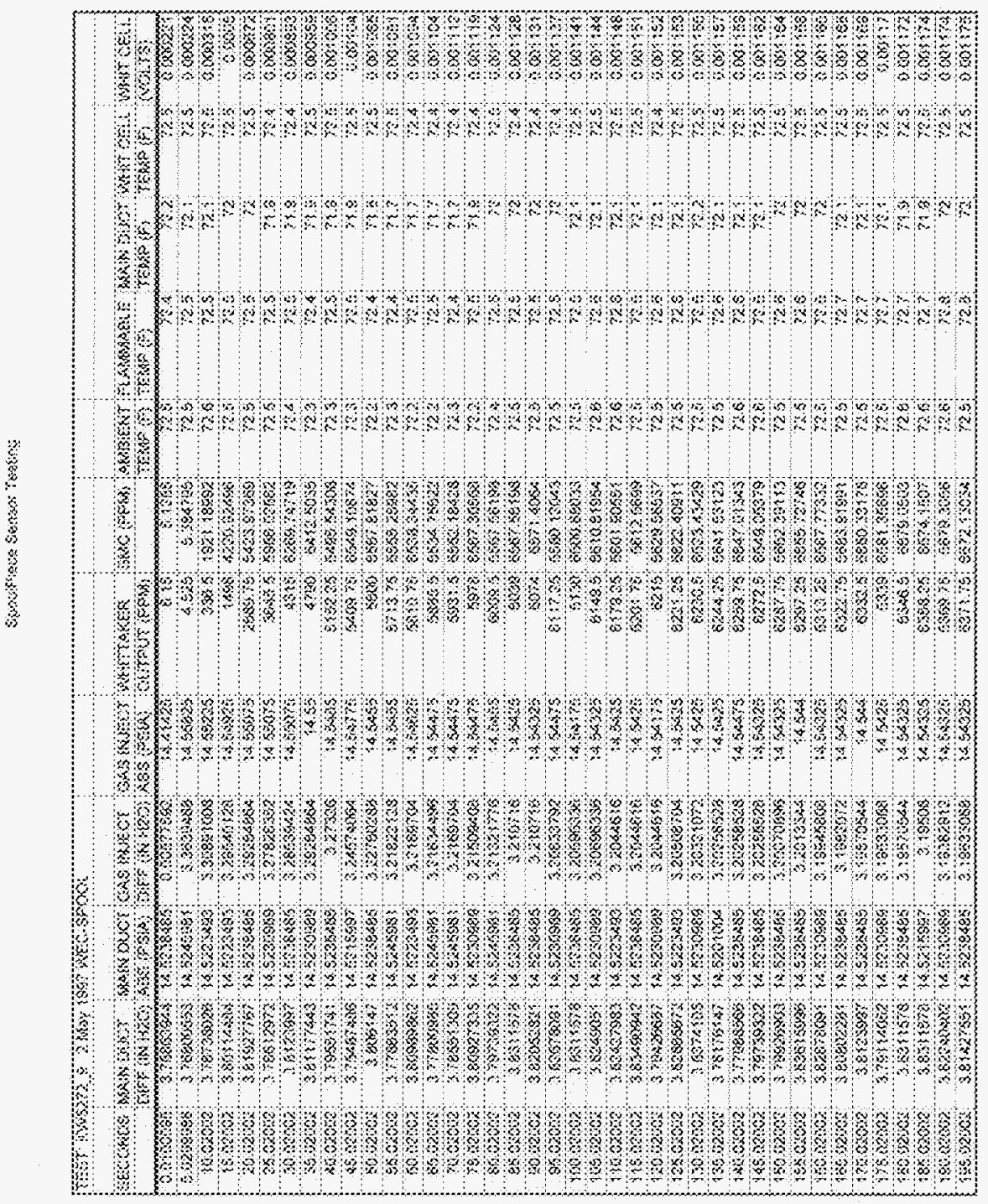




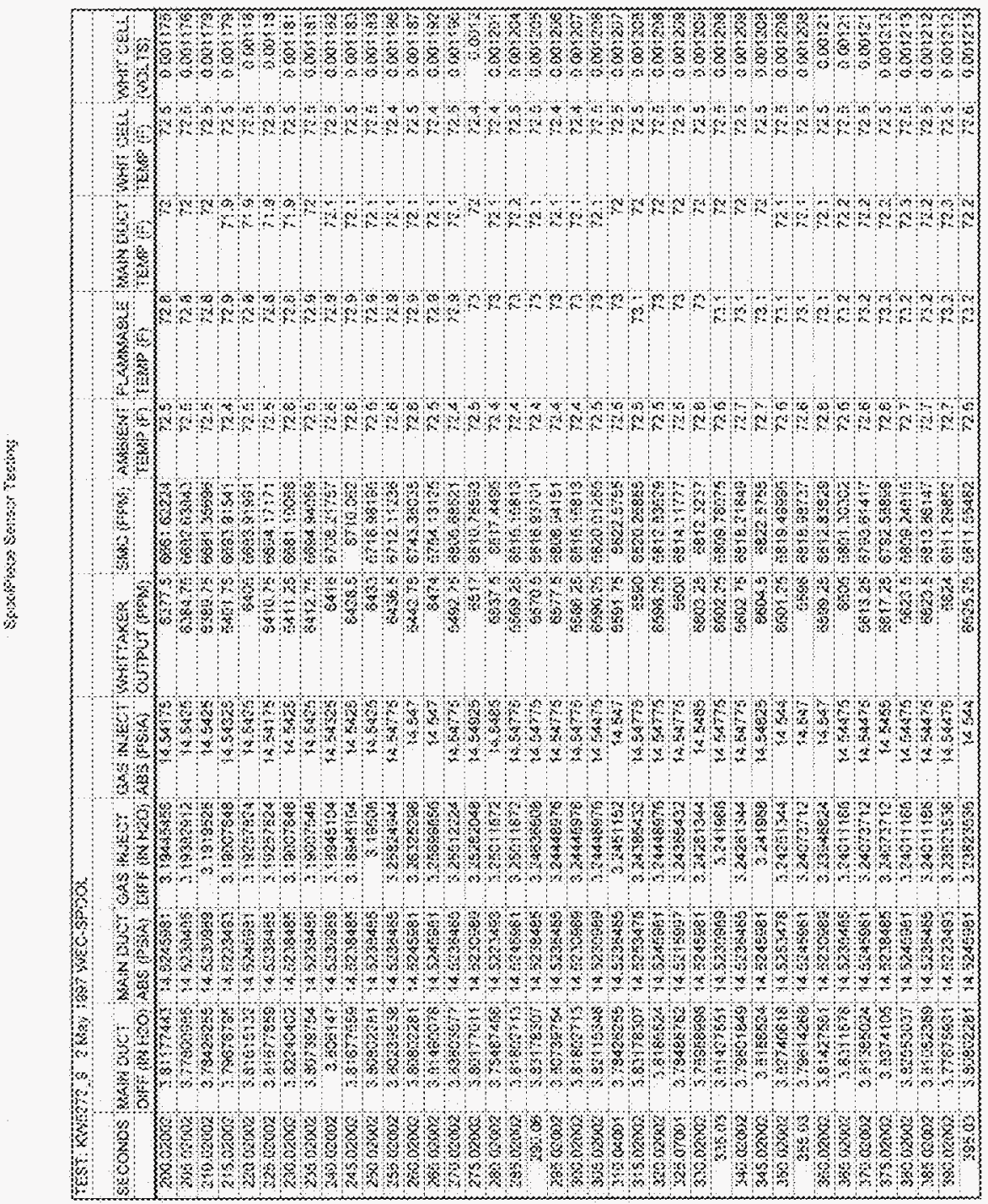


\{NF-SD-WM-TST-276

Poy is

Pro: 16

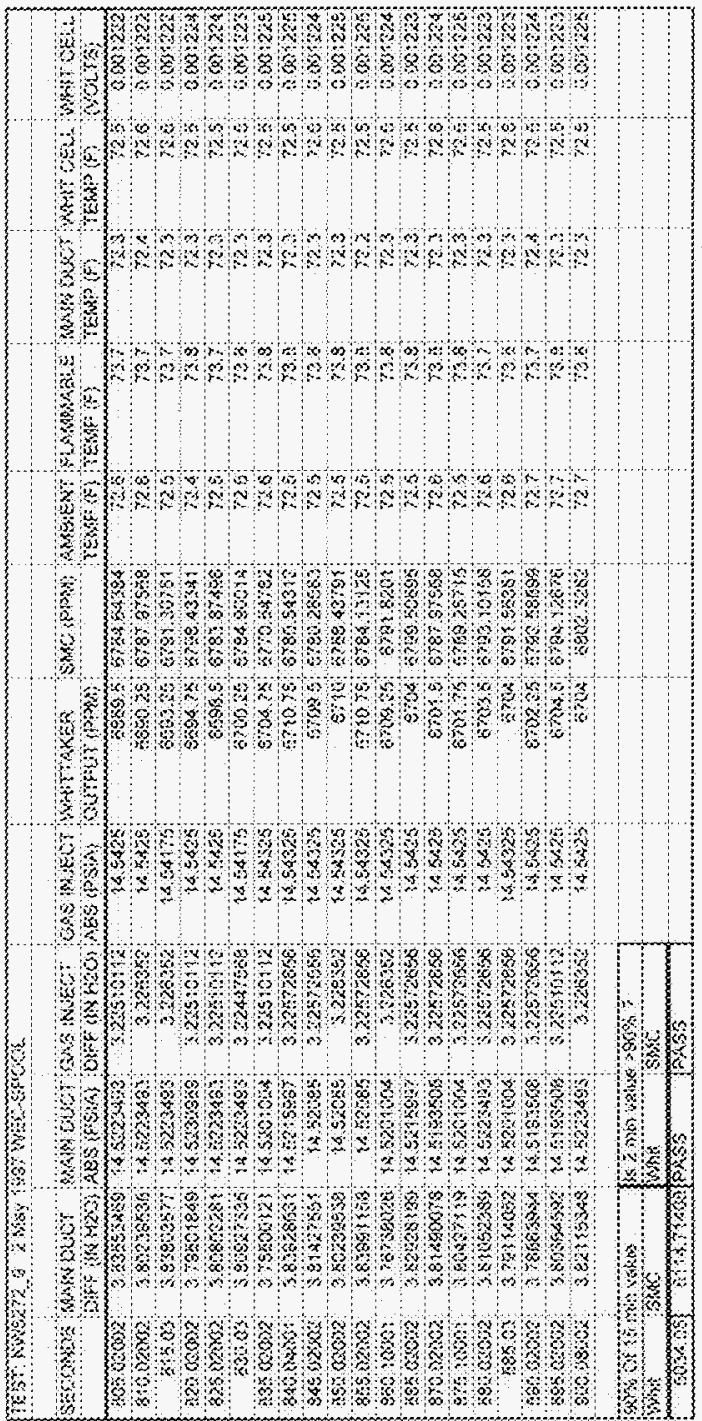




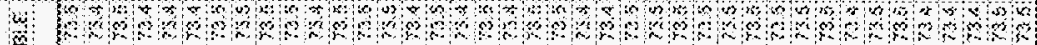
帘

3

के

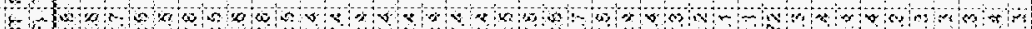

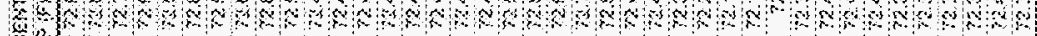
这

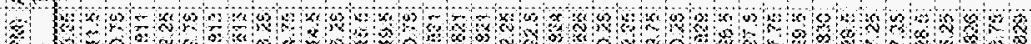

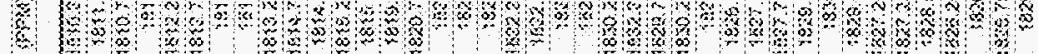

㲖

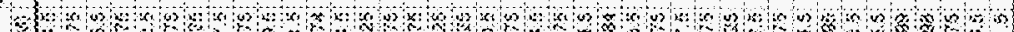

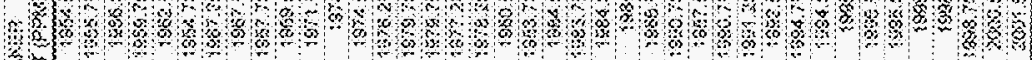

(3)

2

28

Wow Womb

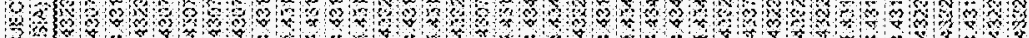

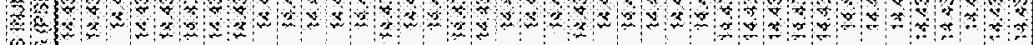
(3)

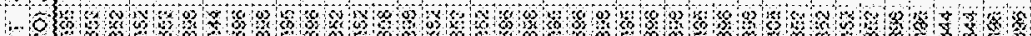

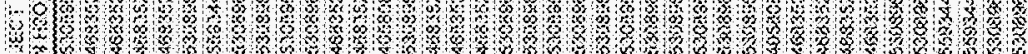

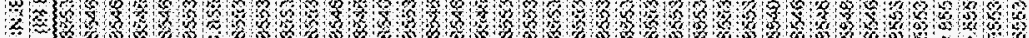

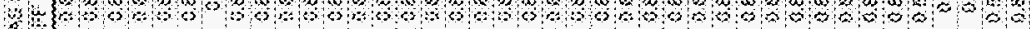
3.3

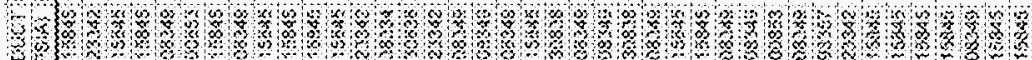

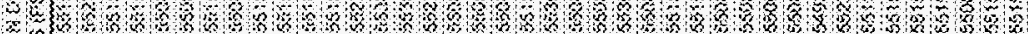

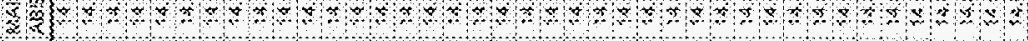

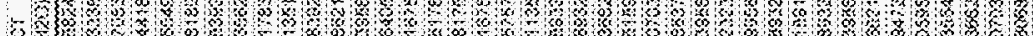

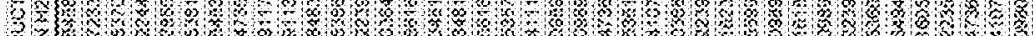
2.

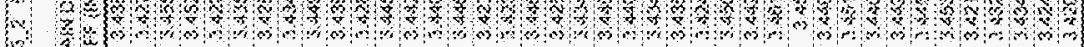

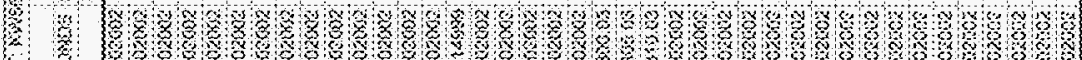




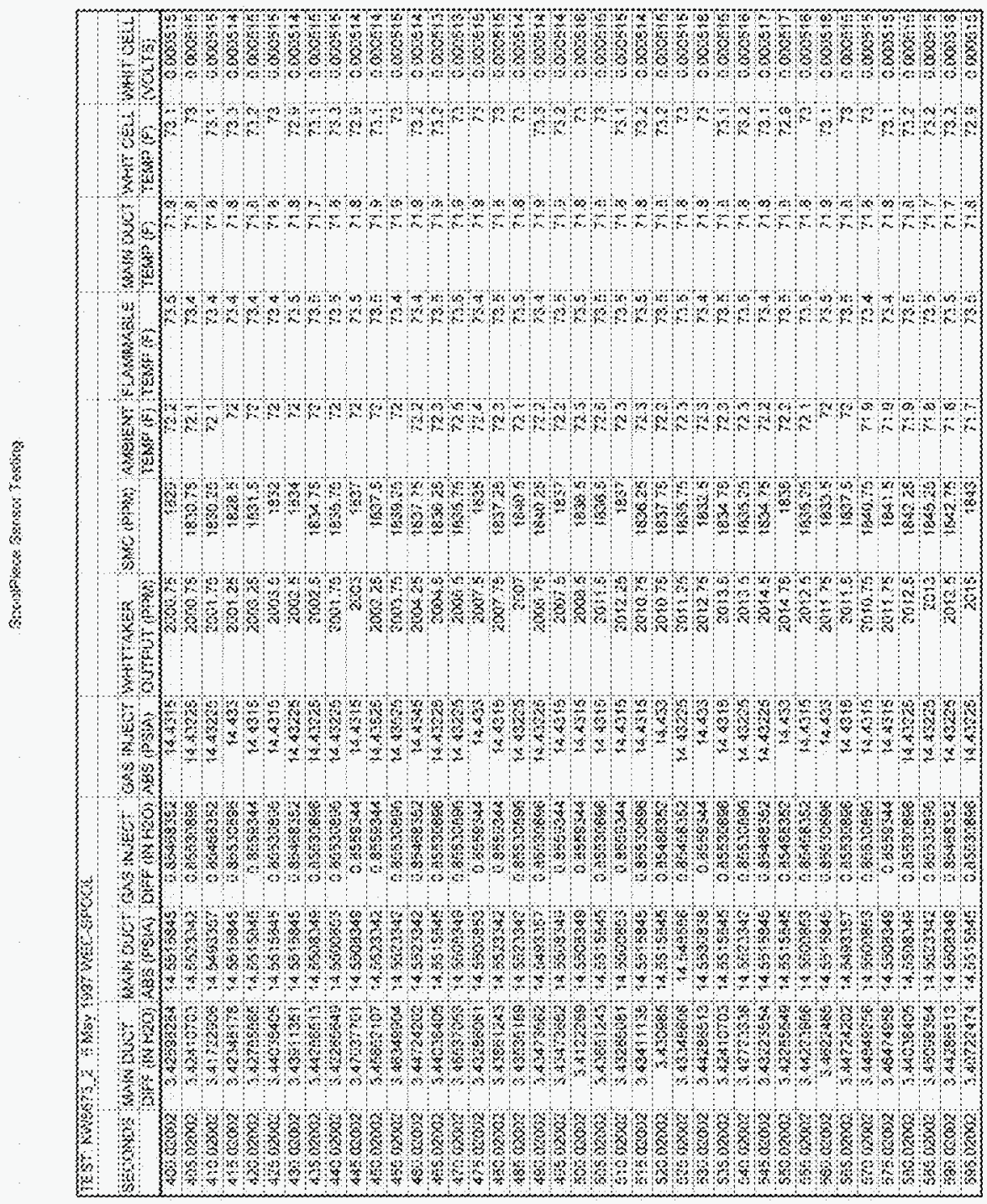




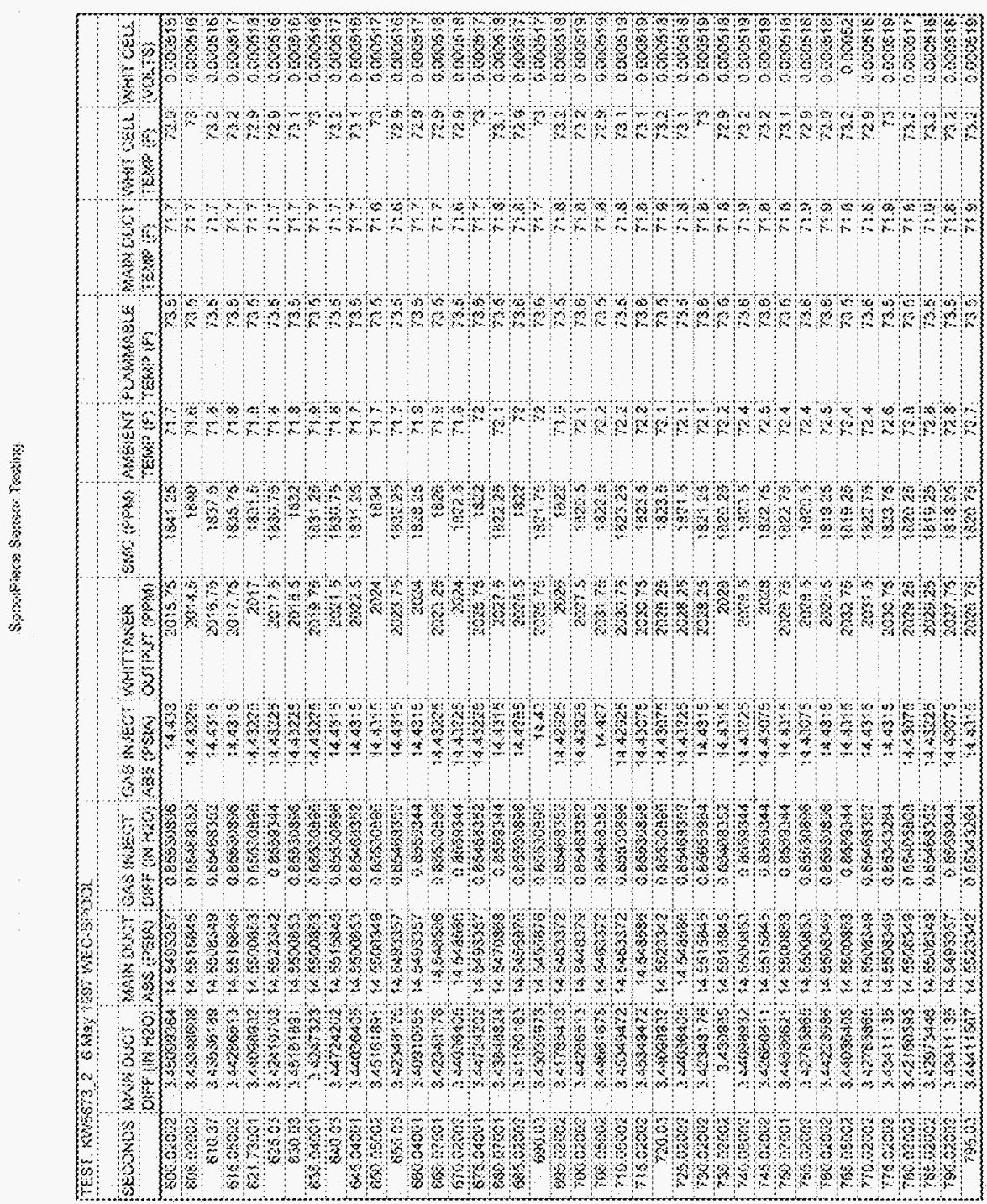




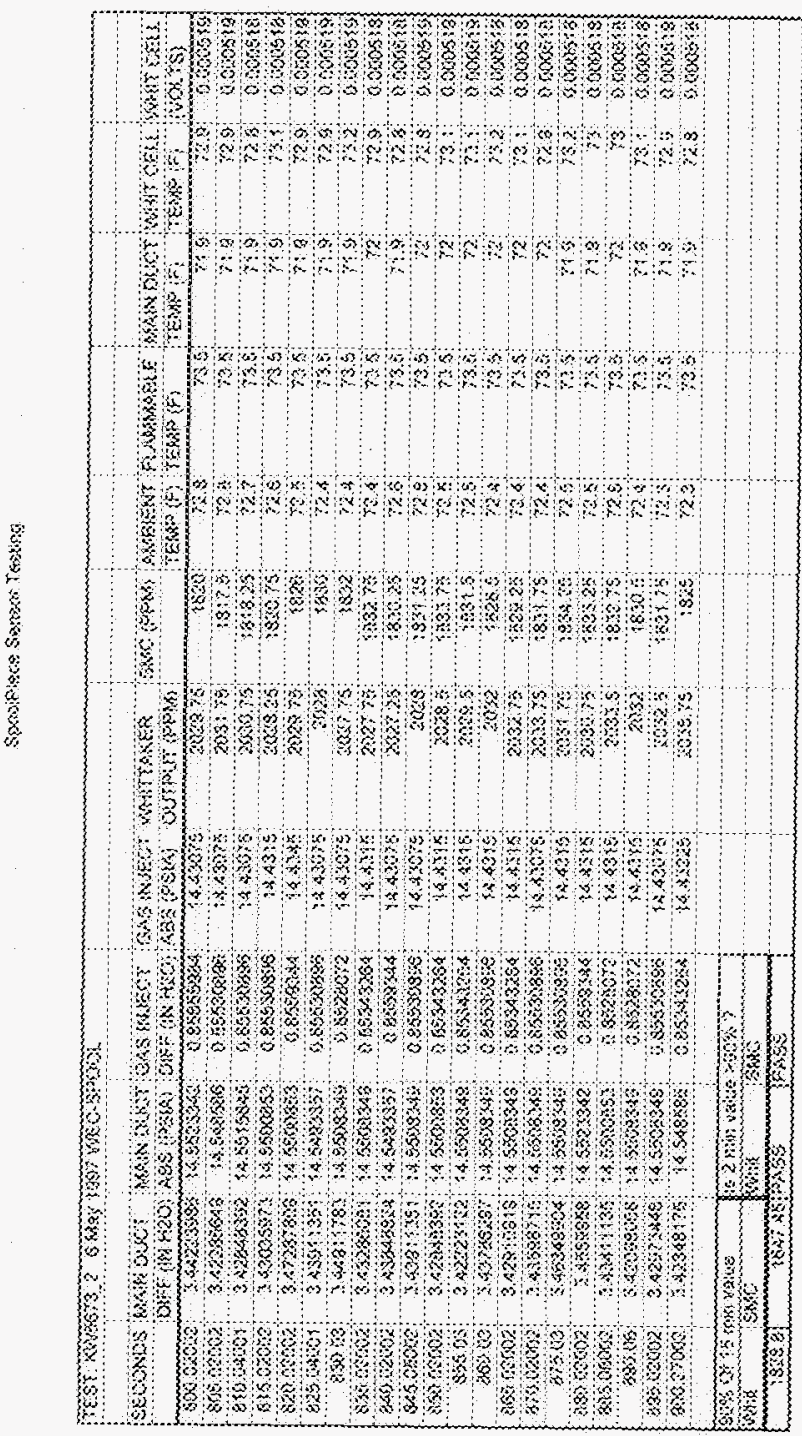




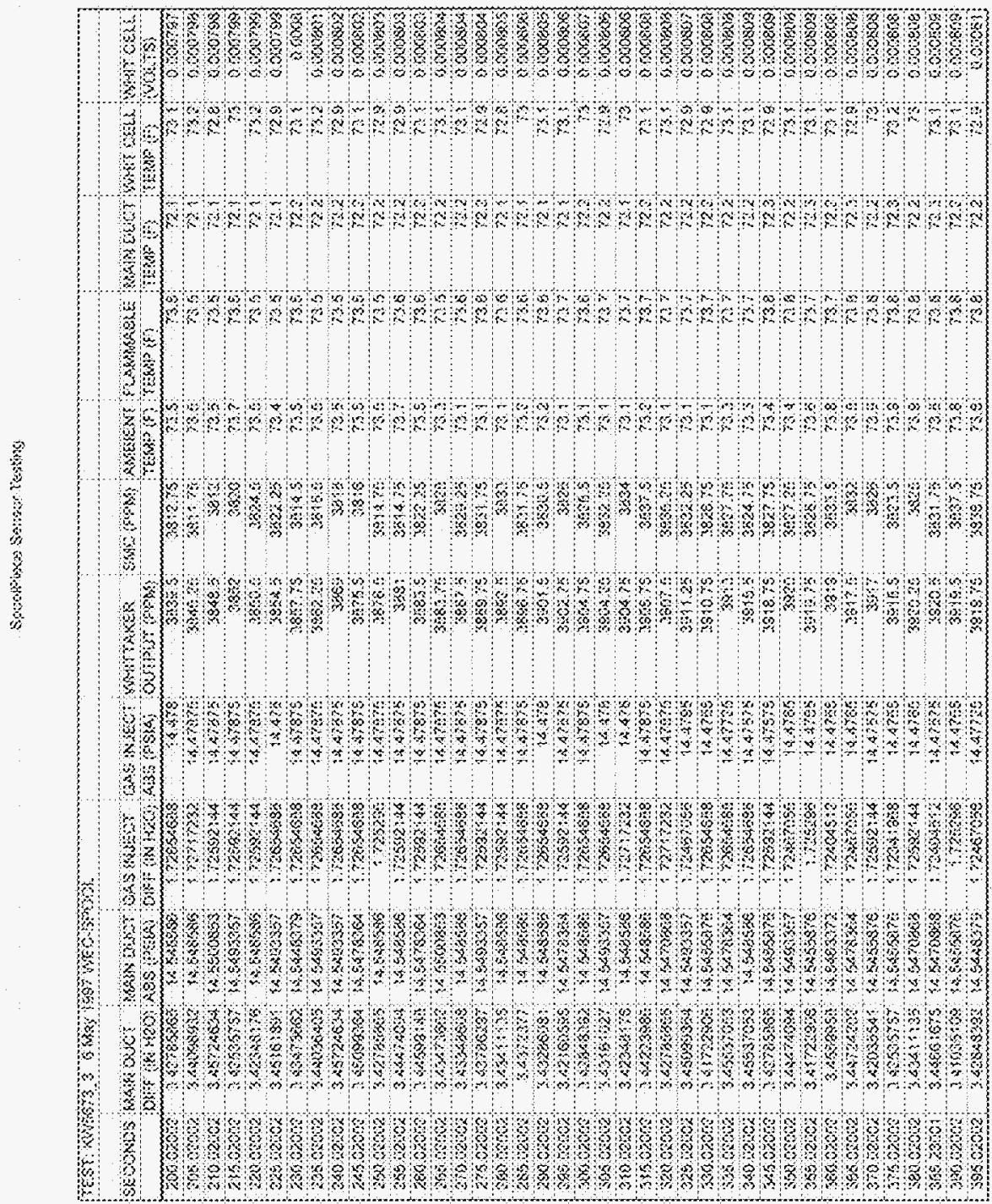




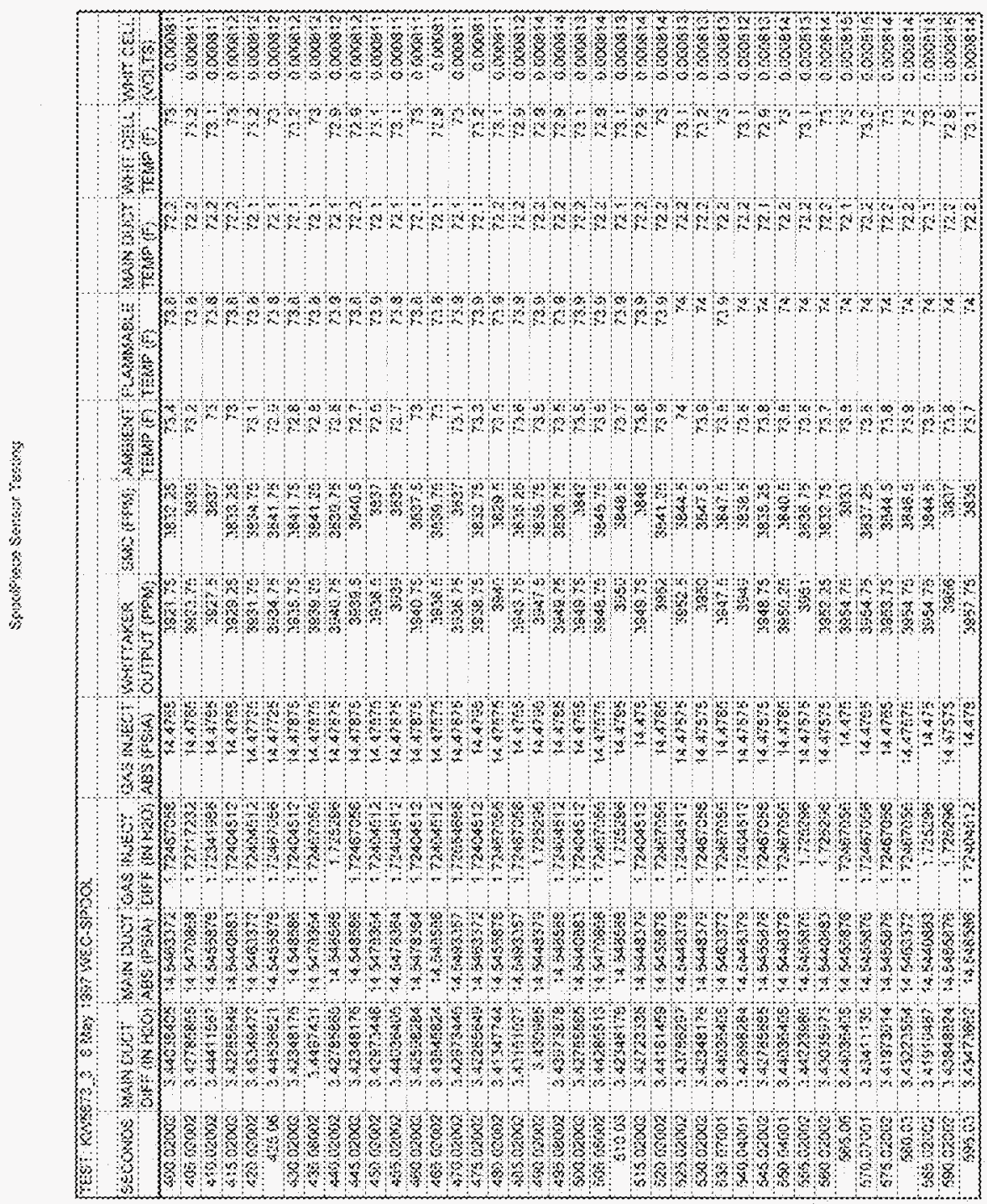




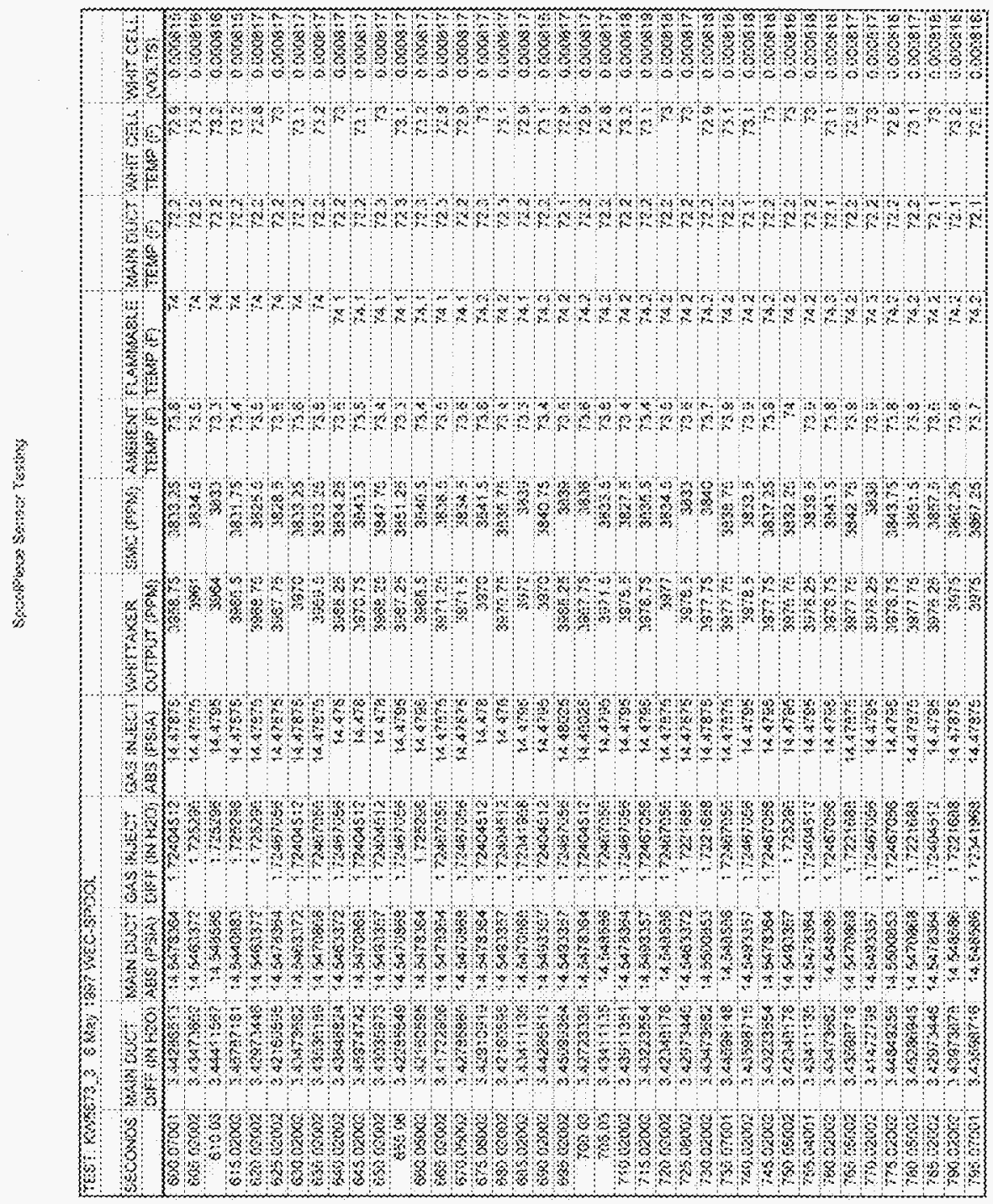




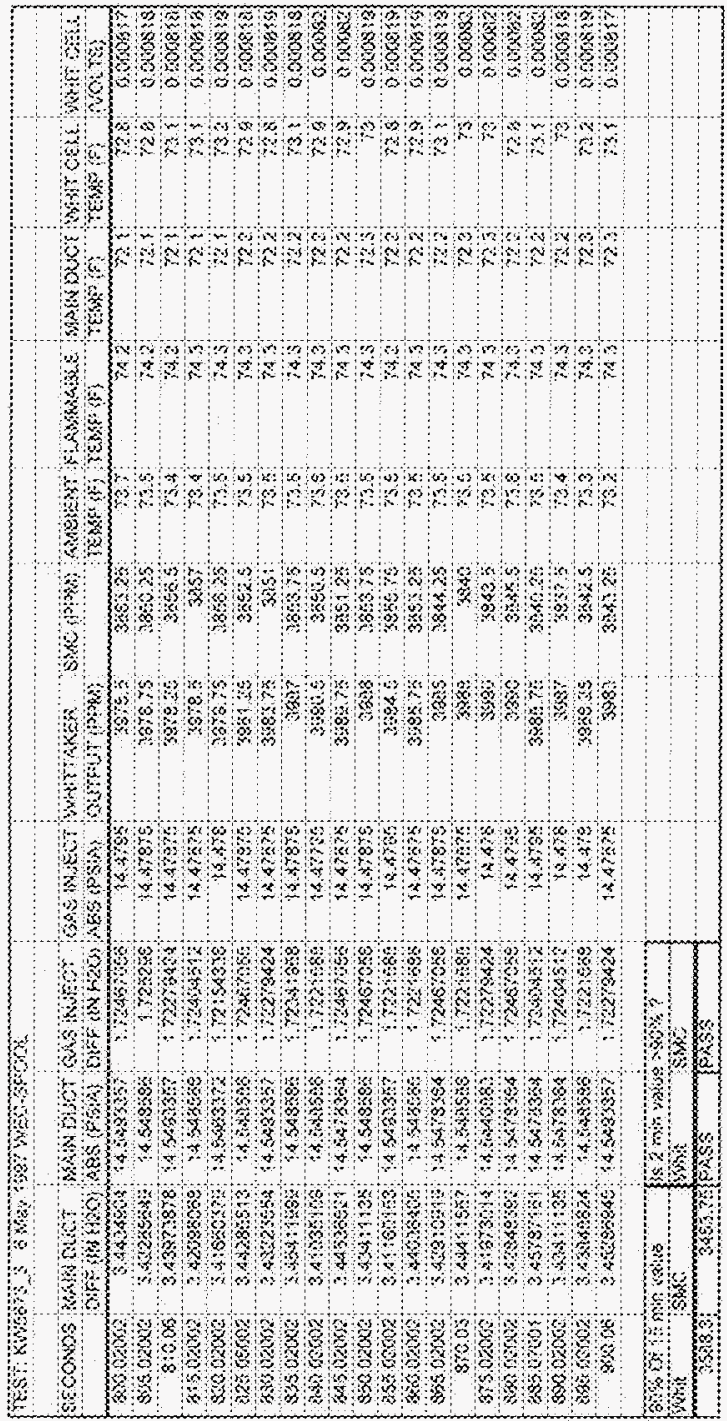




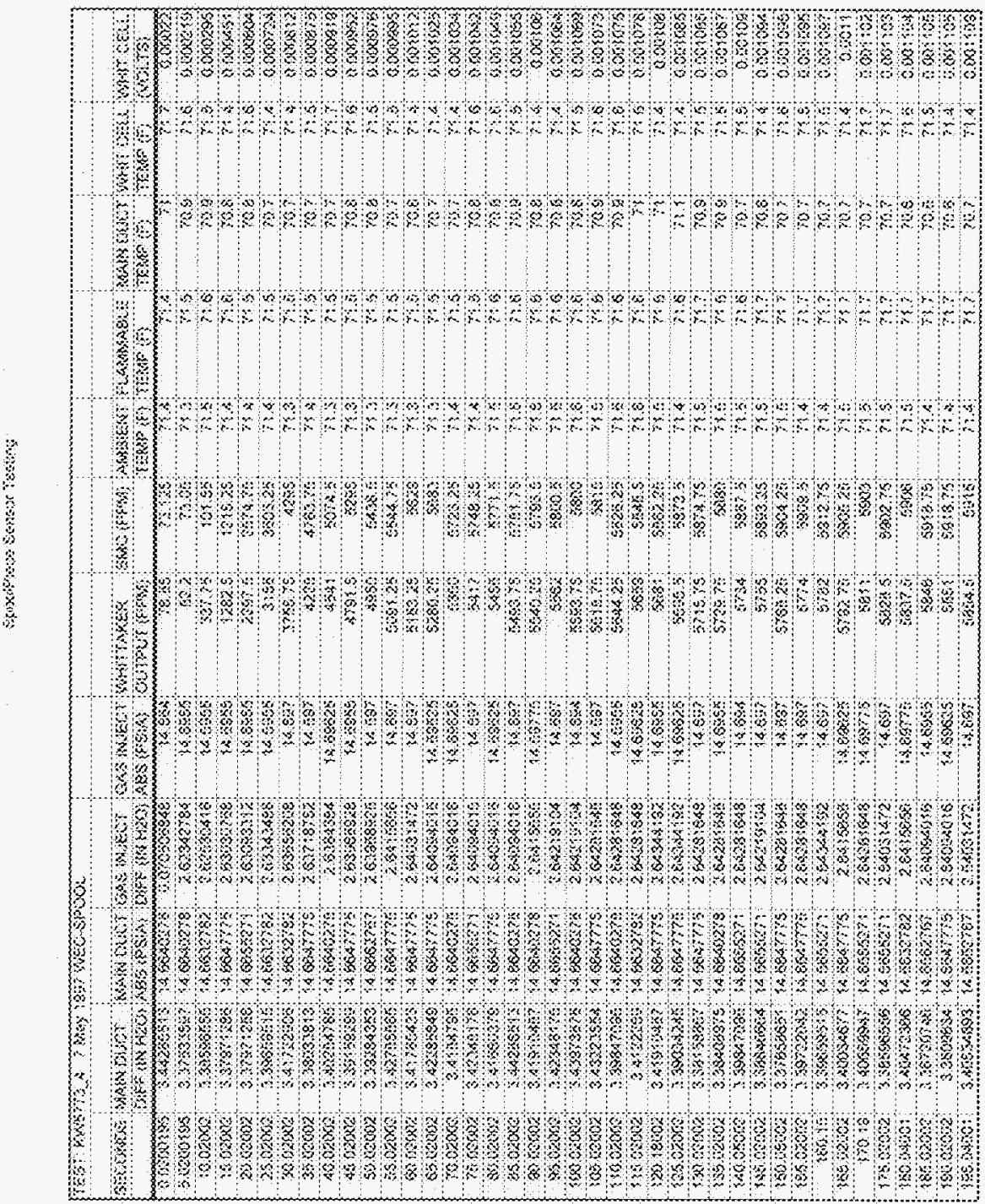




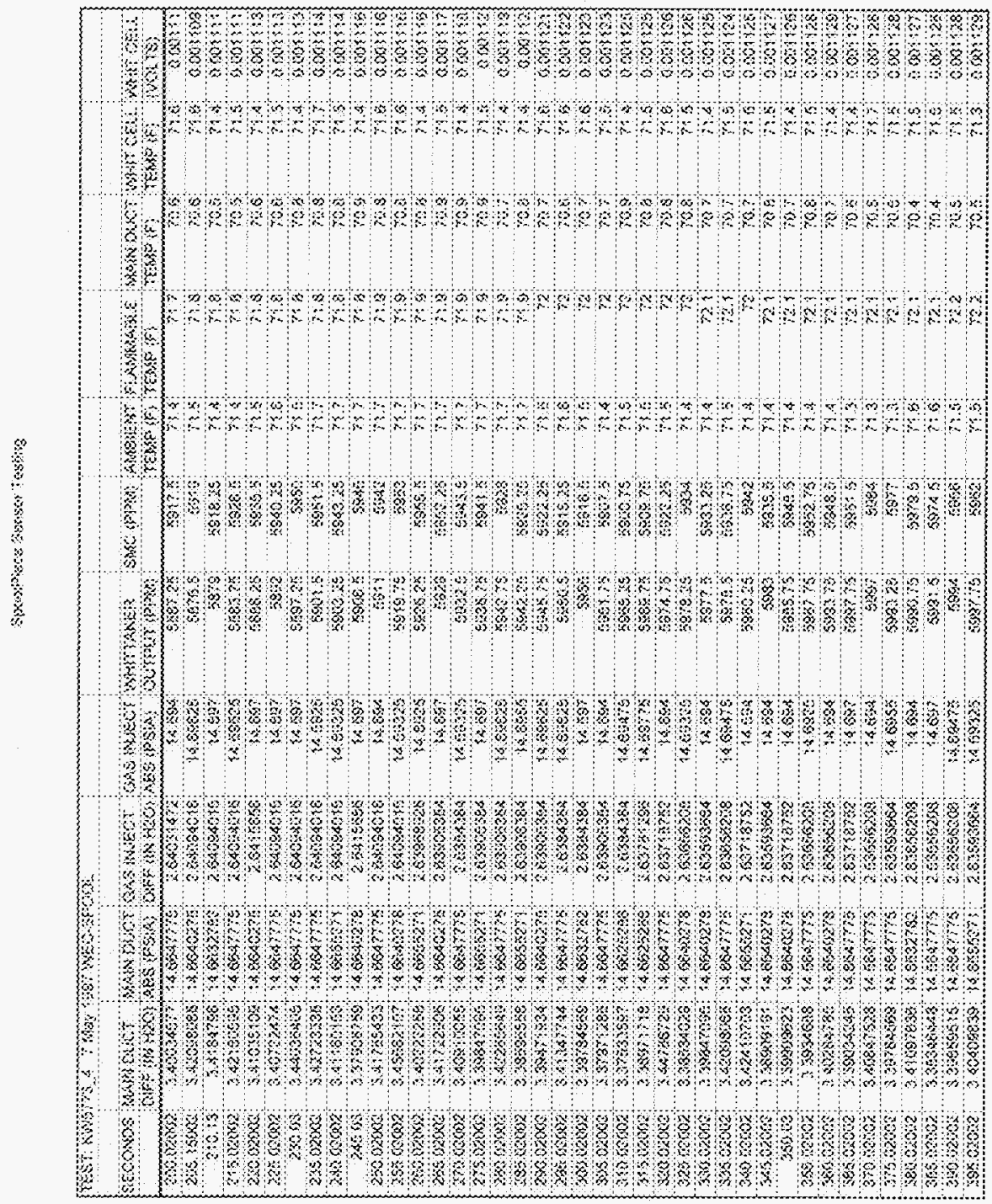




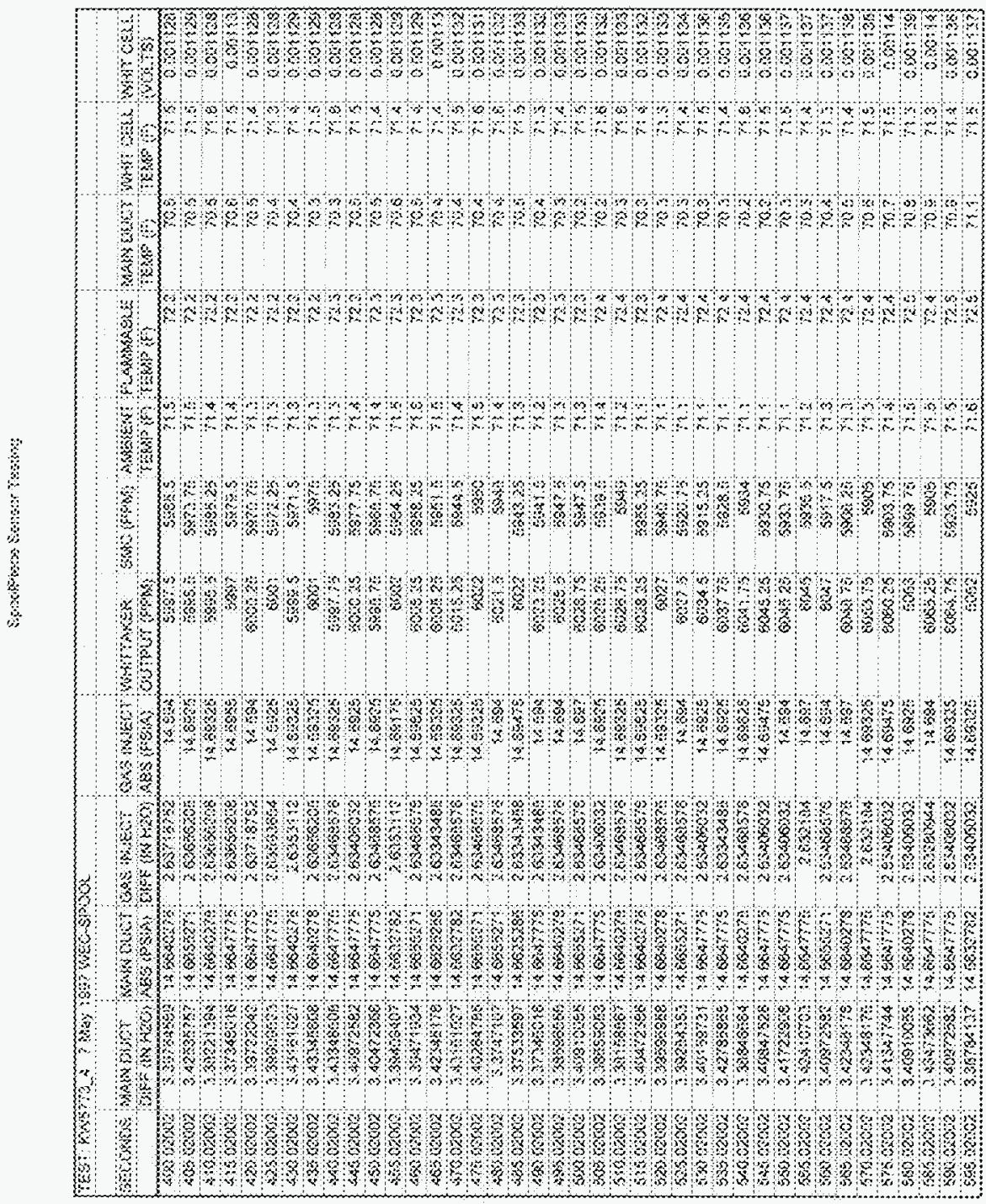




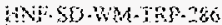

सकरे \{\}

Pros: ins

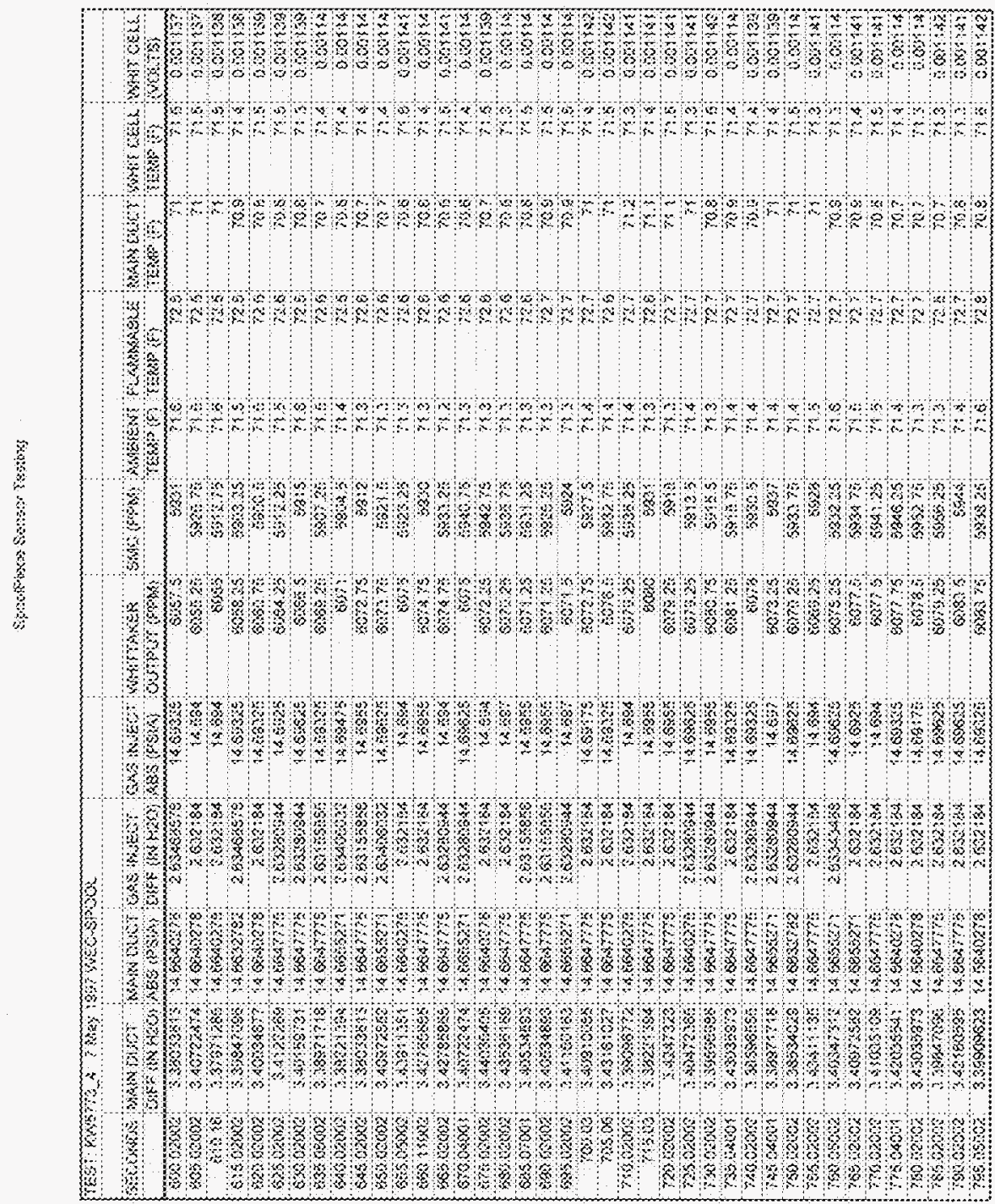




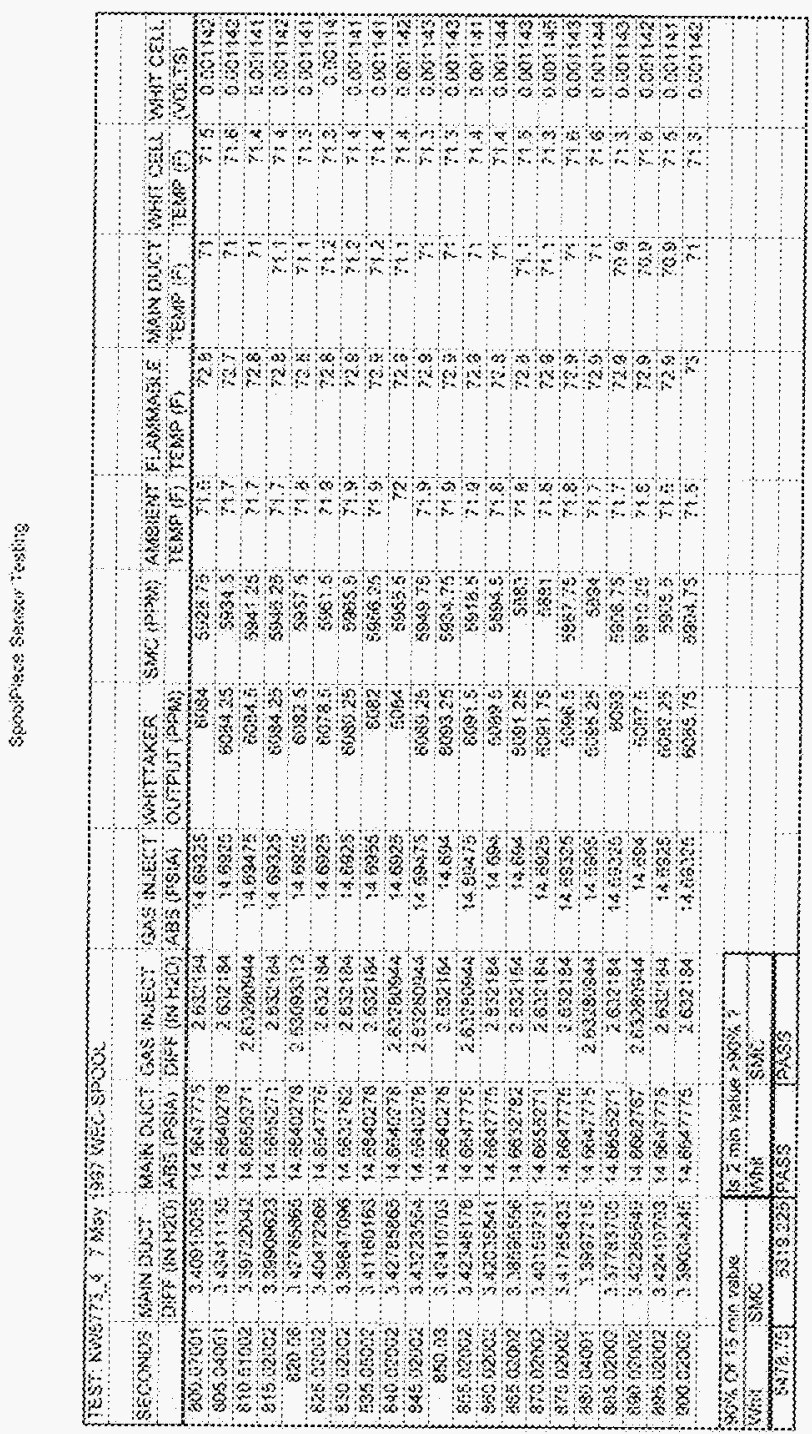




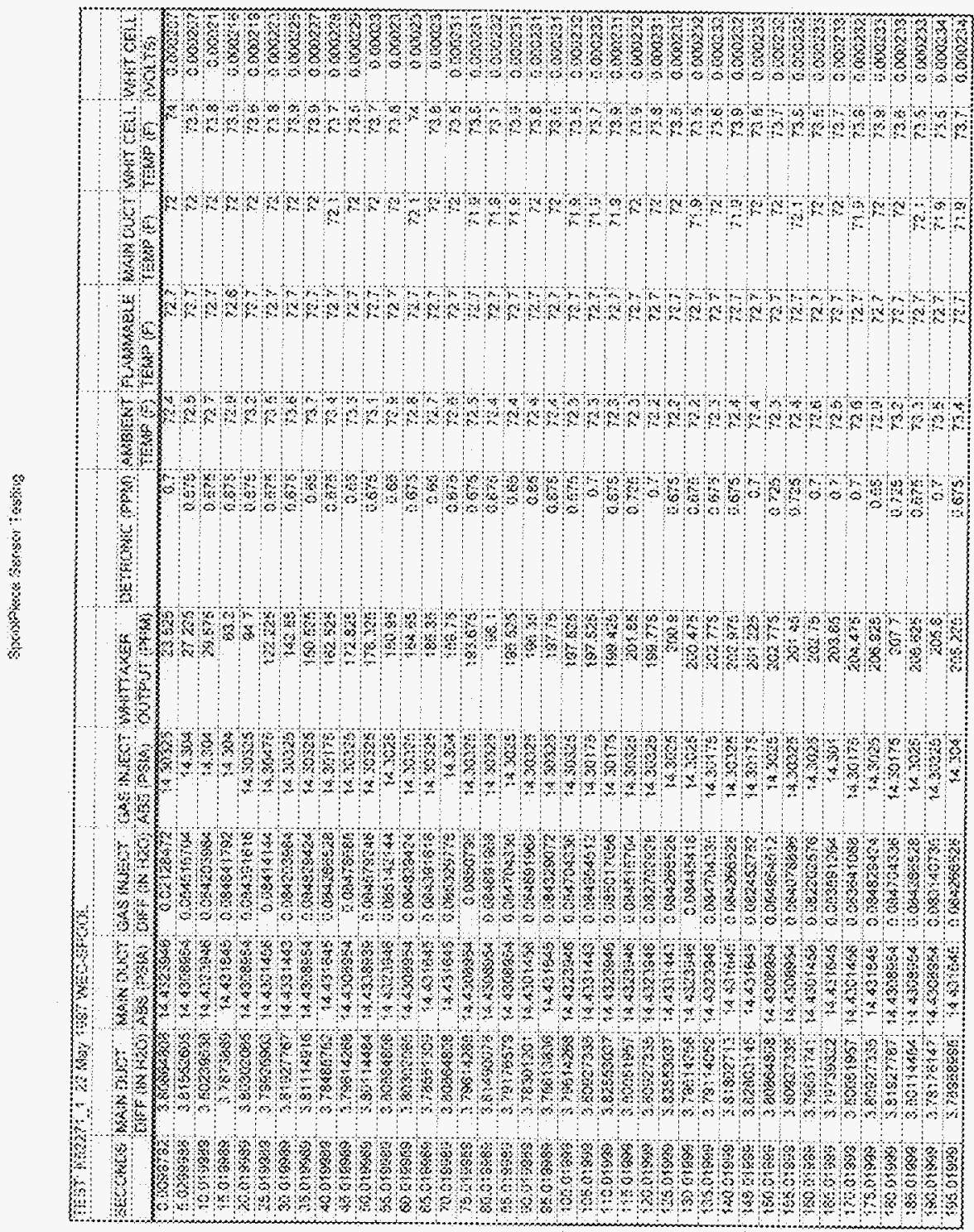




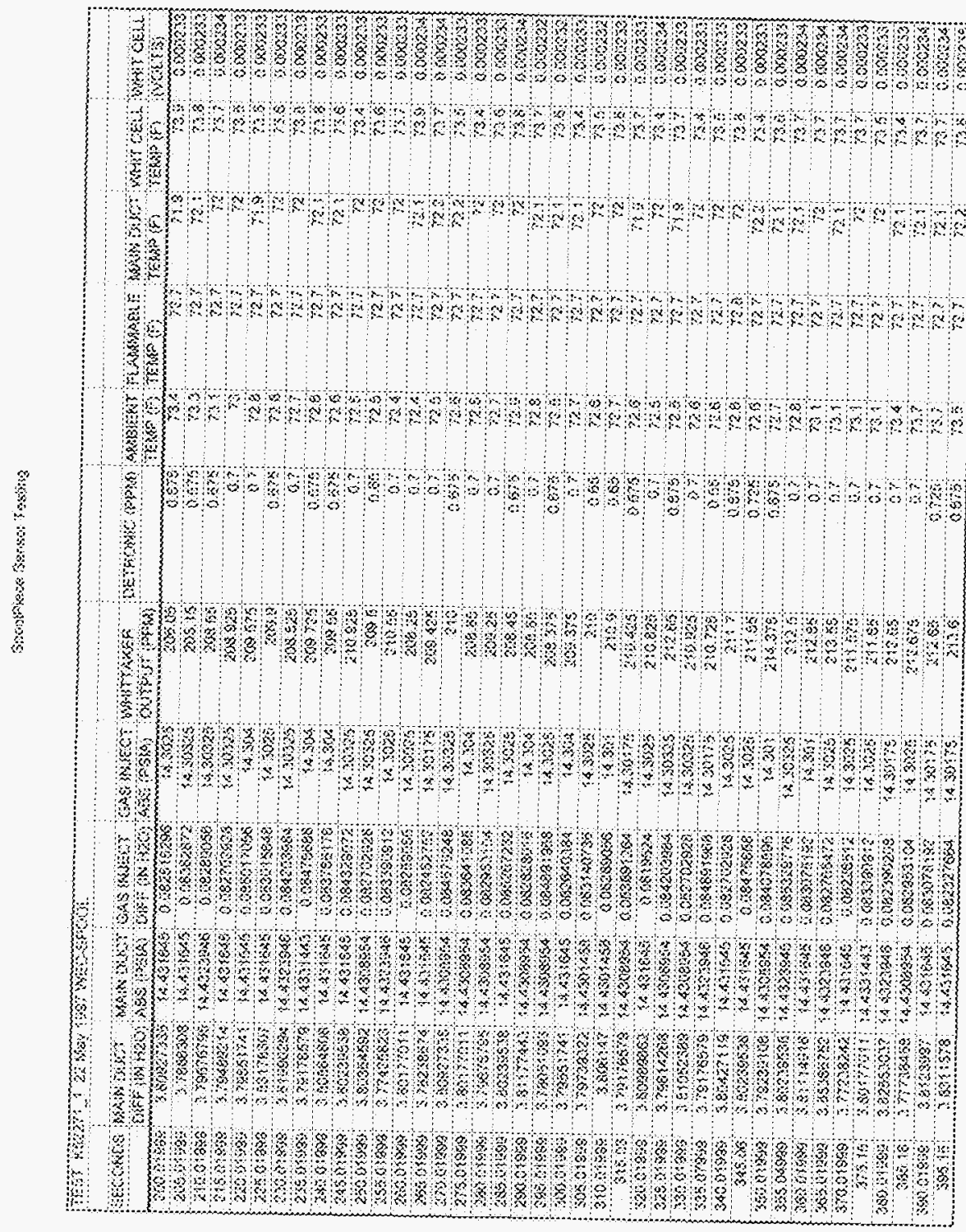




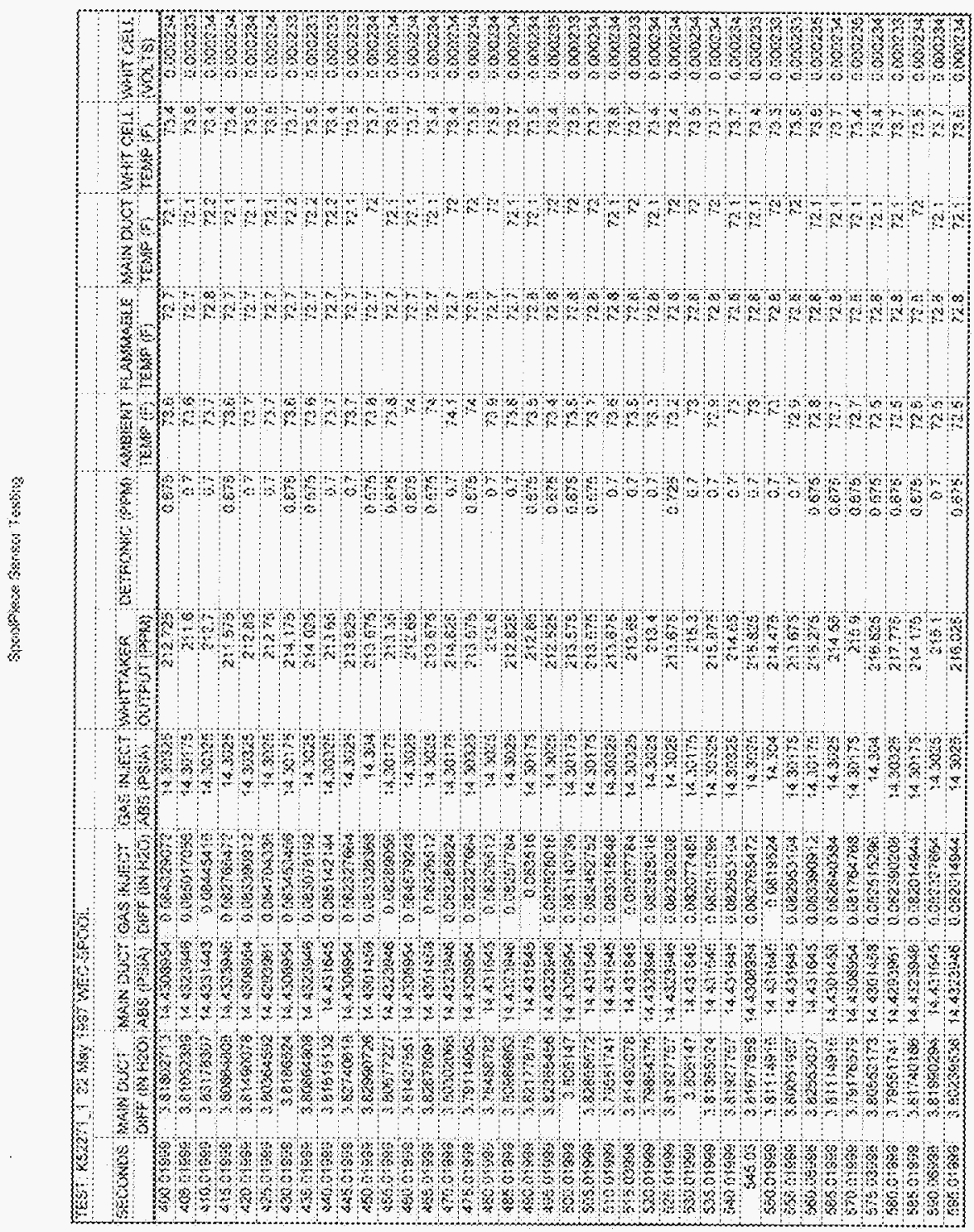




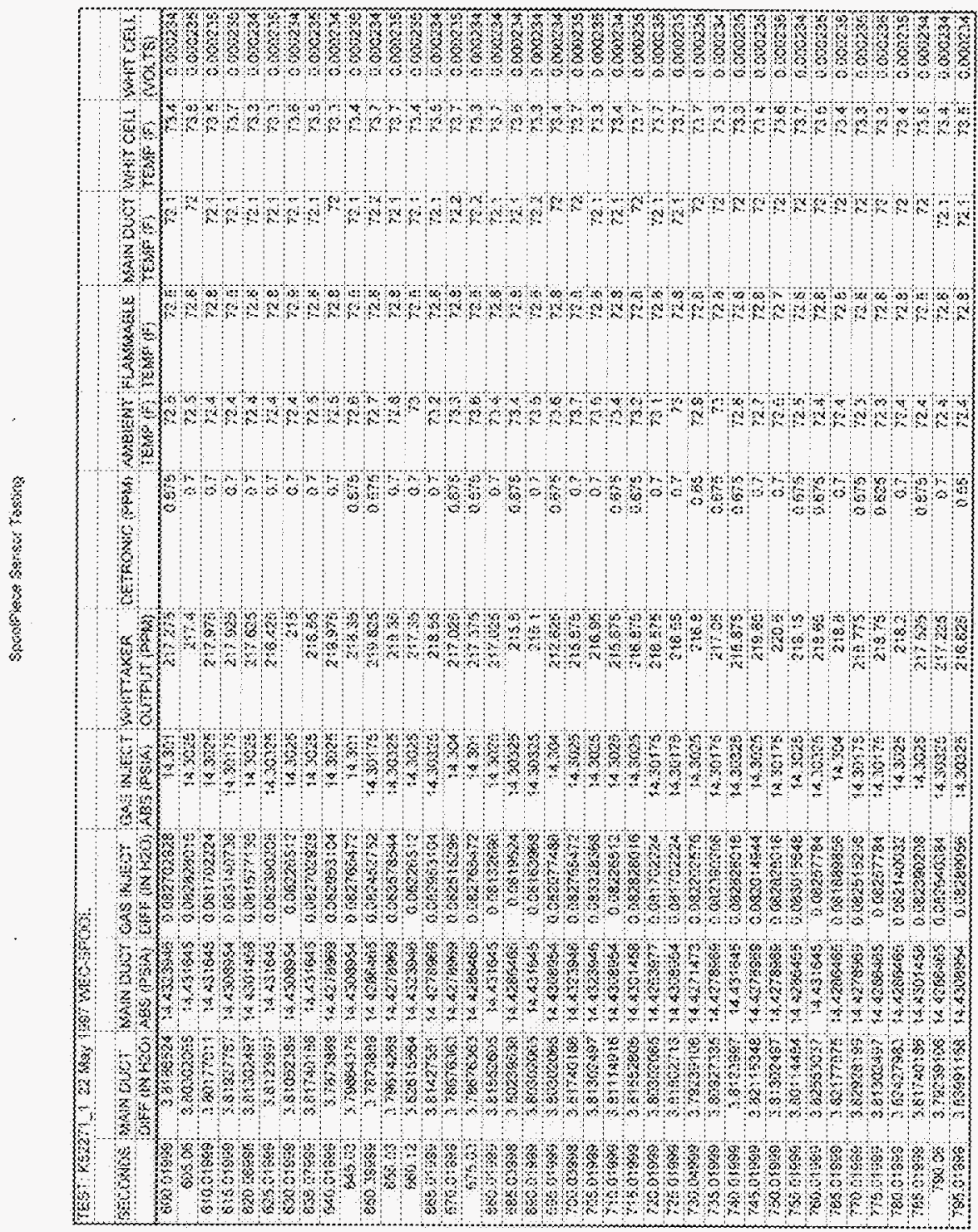




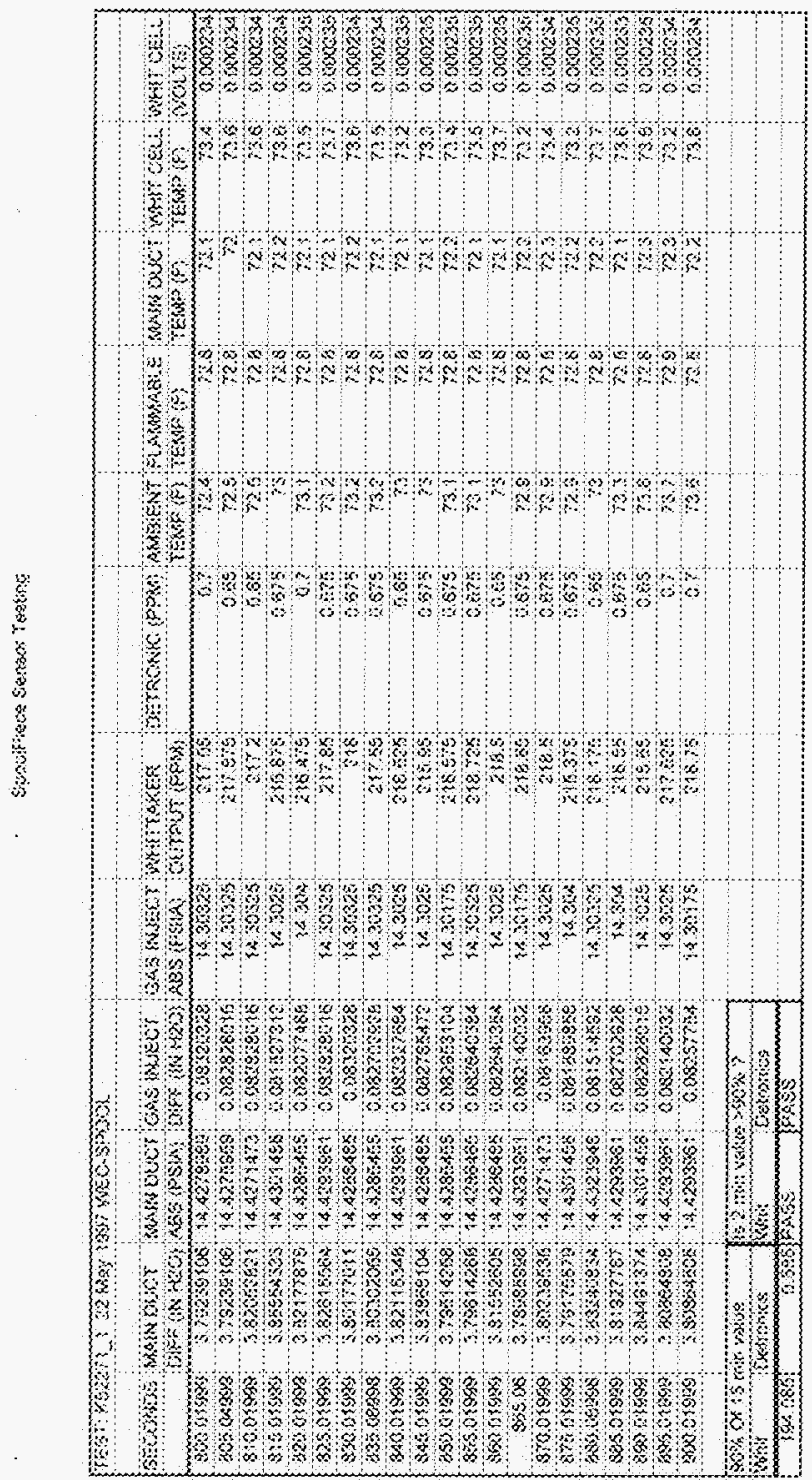




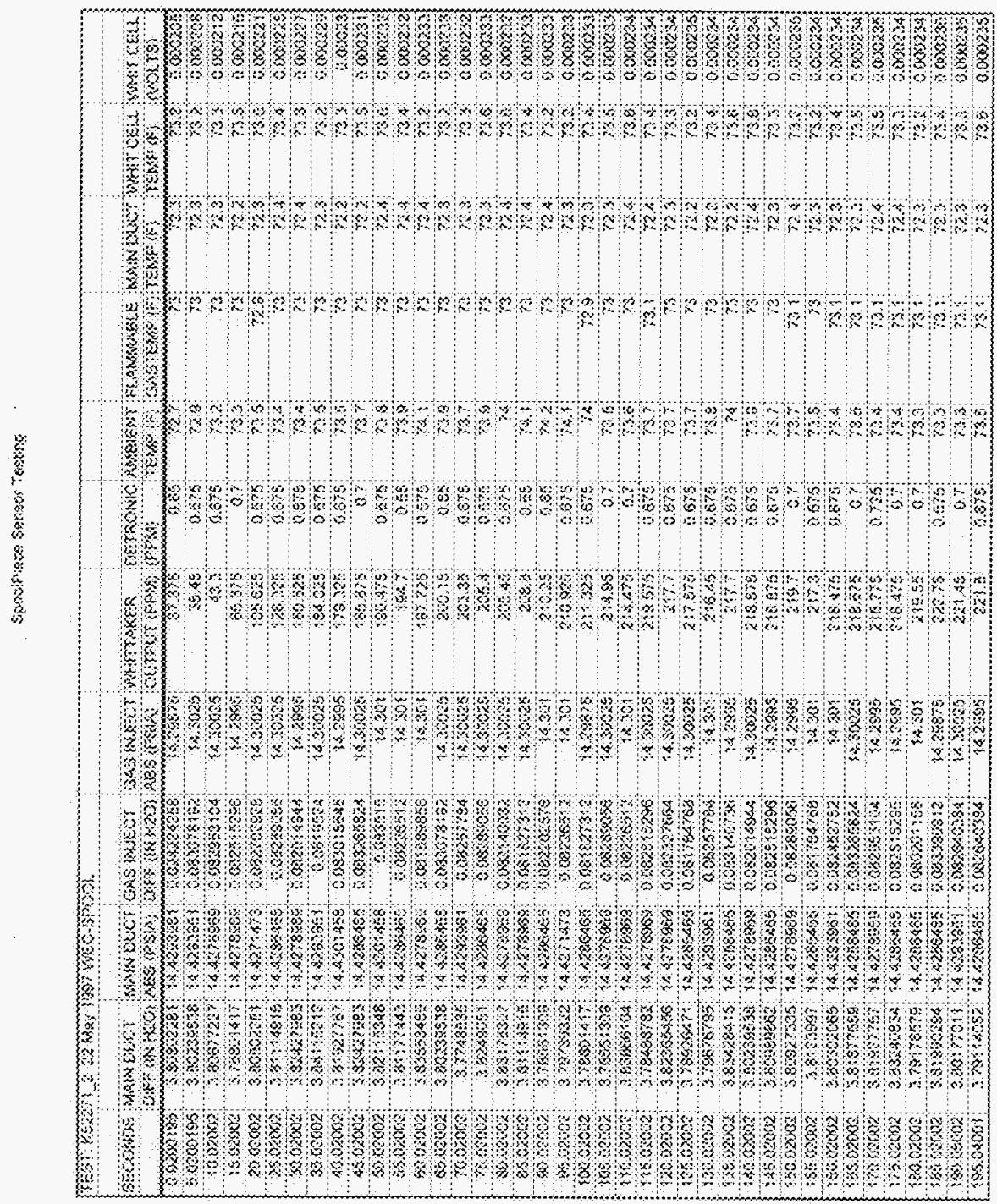




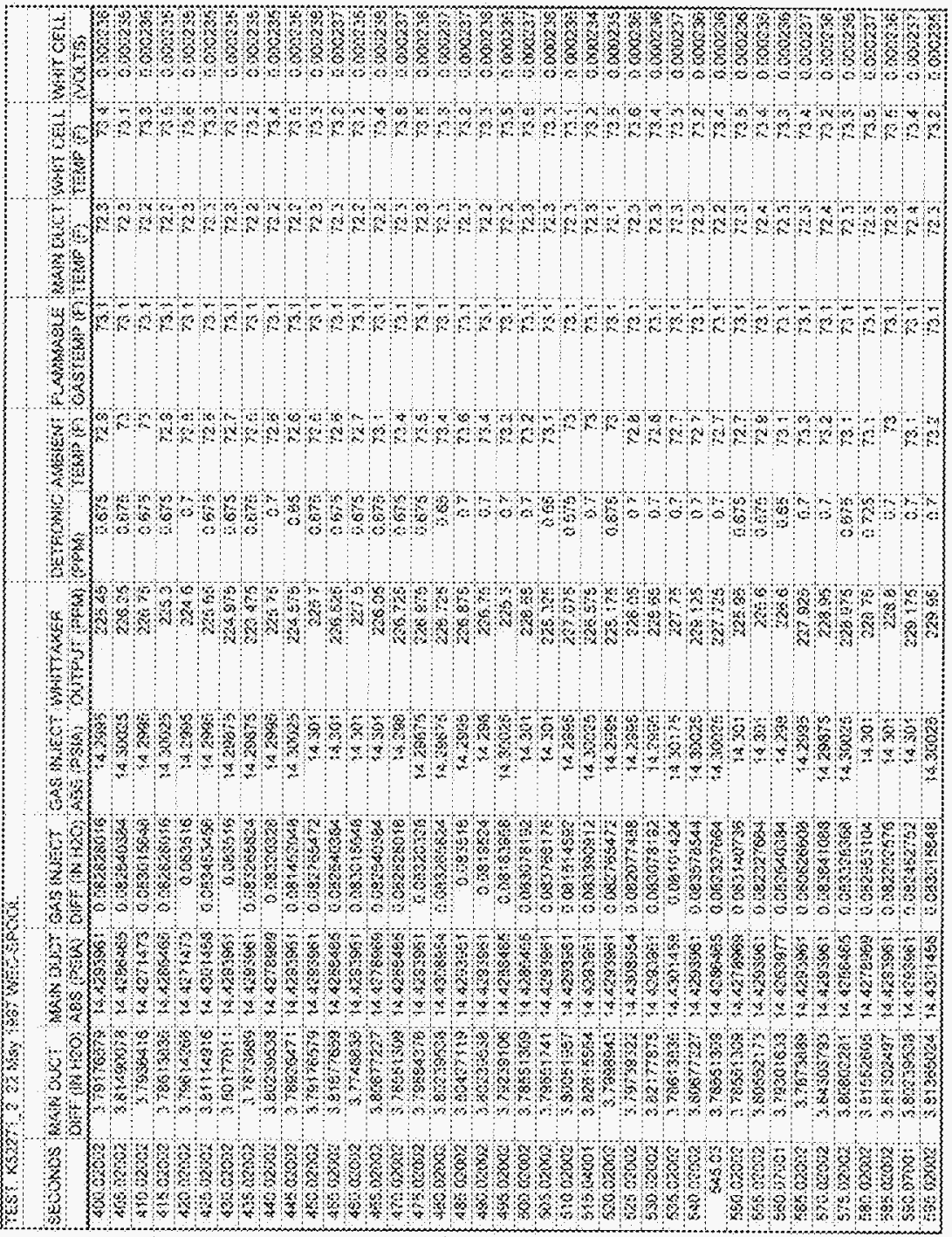




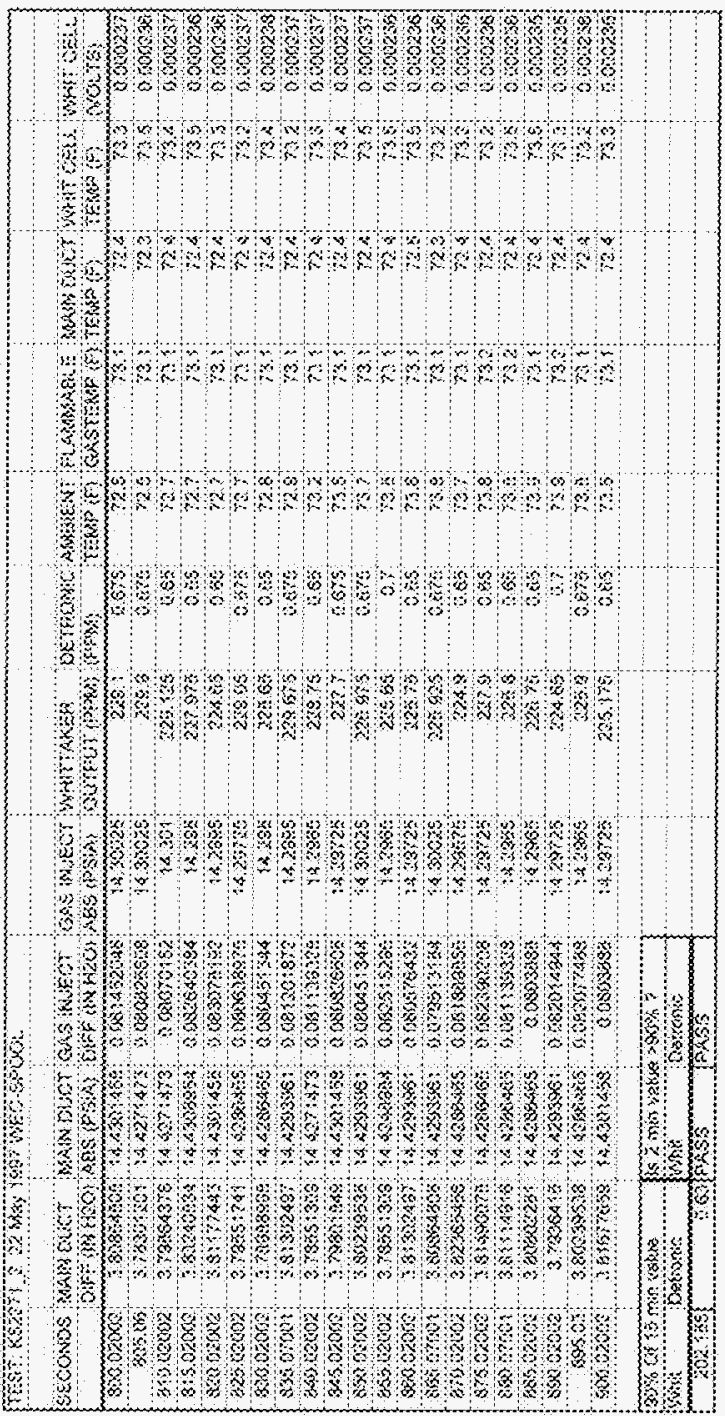




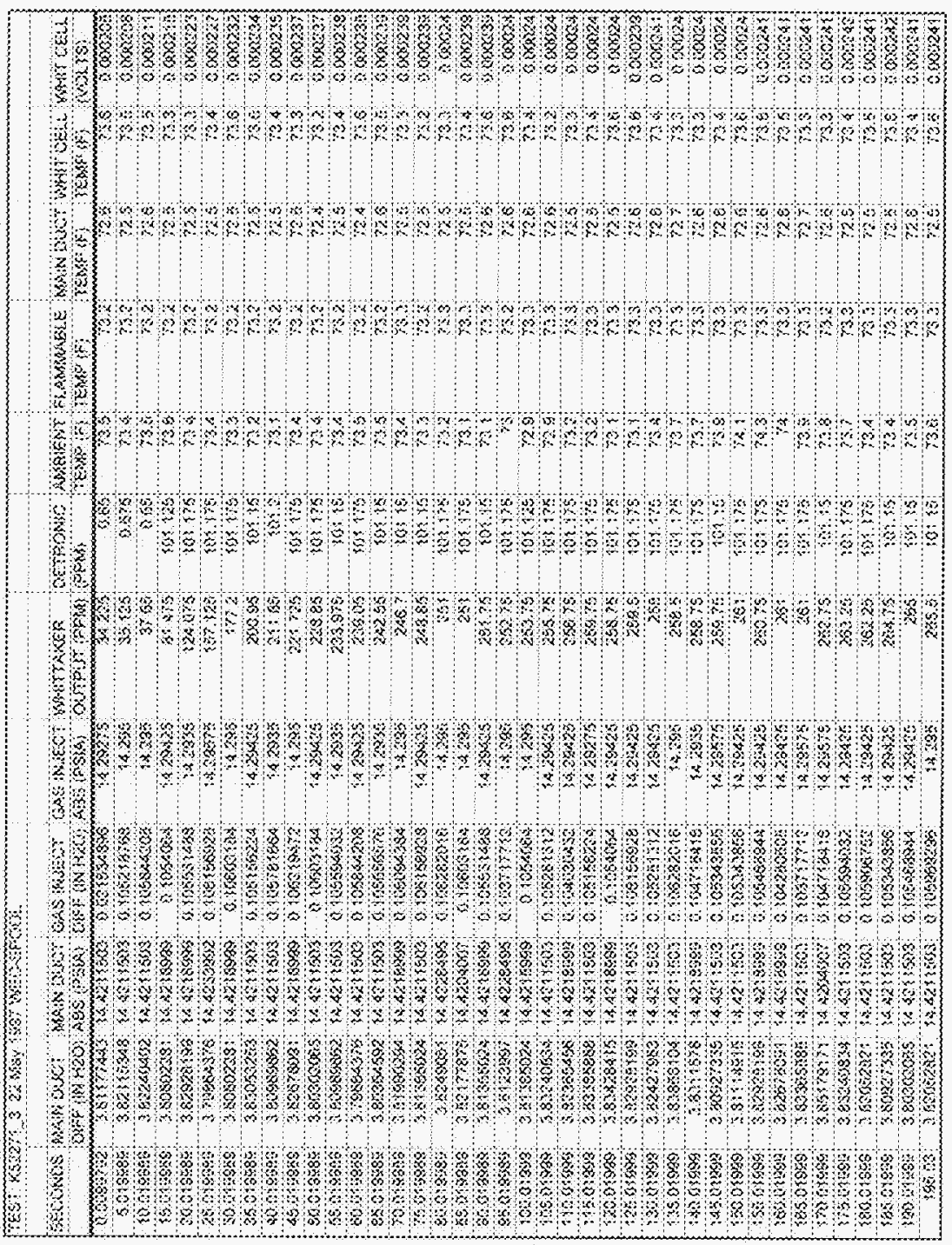




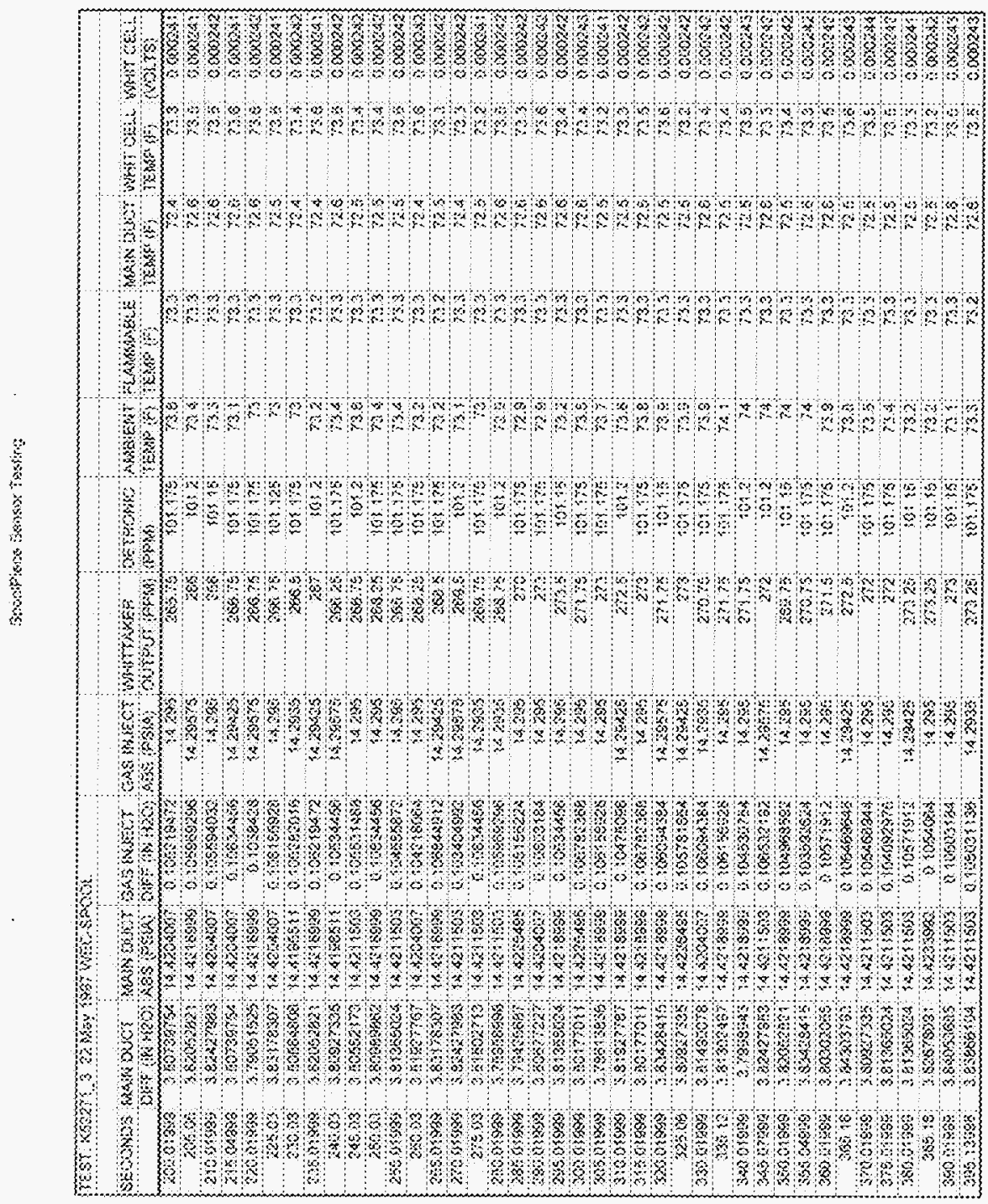




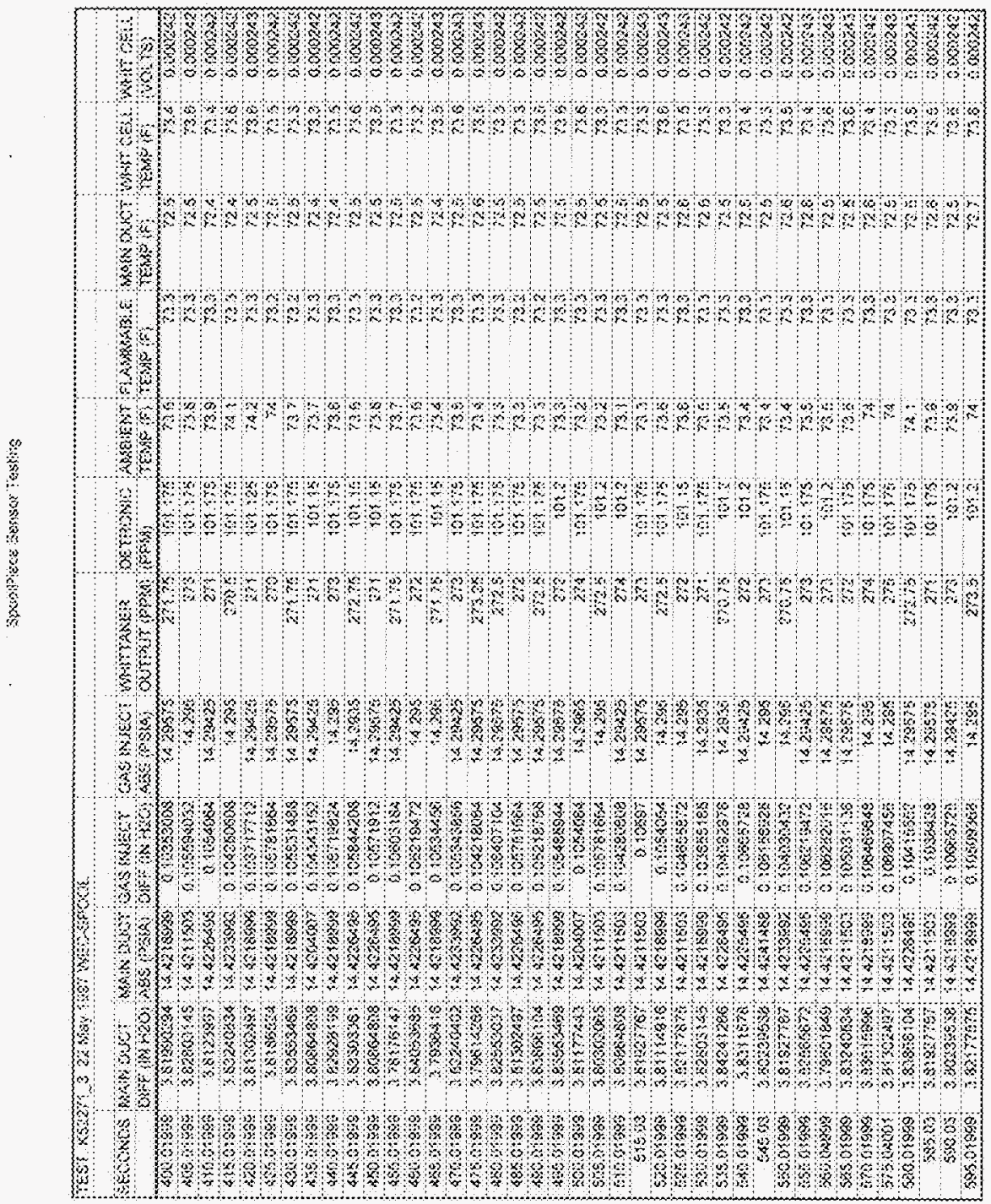




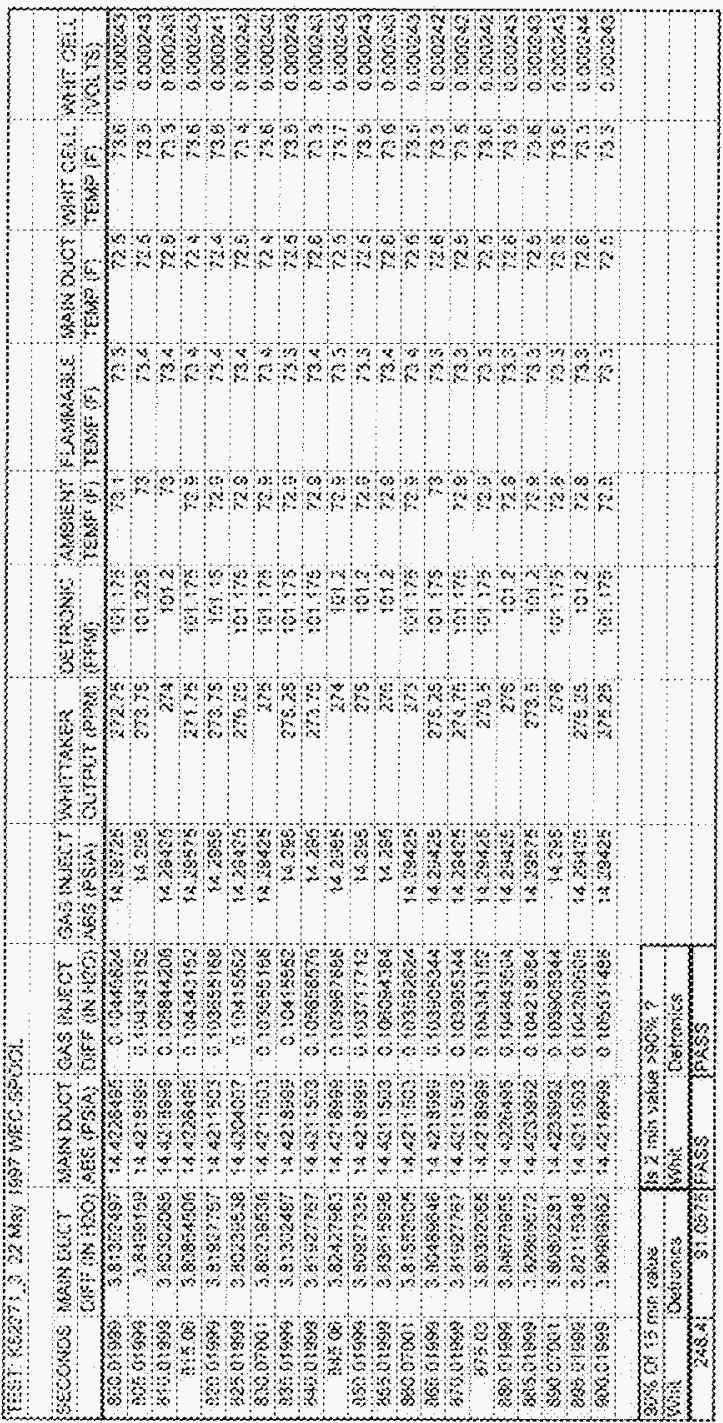




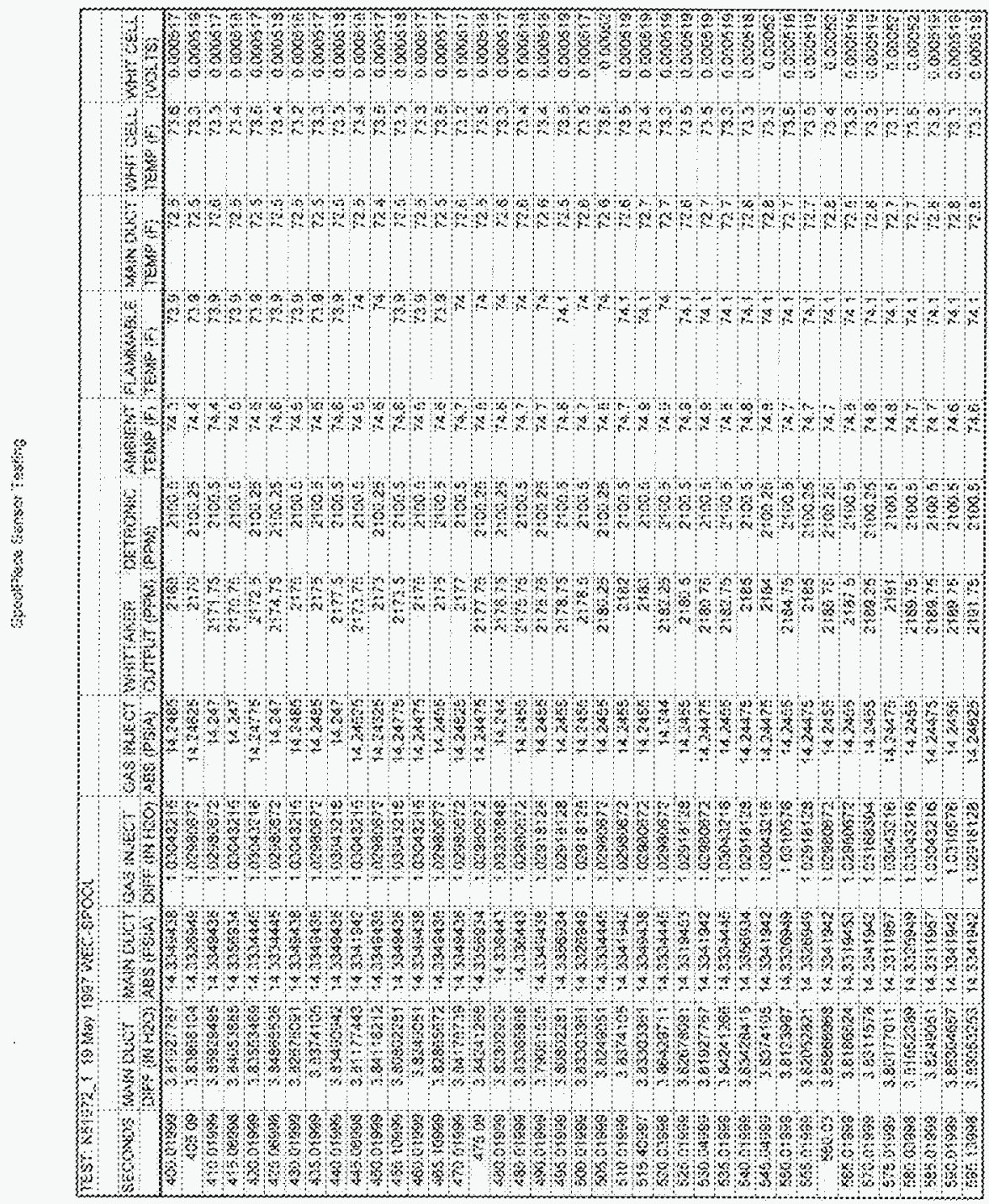


W

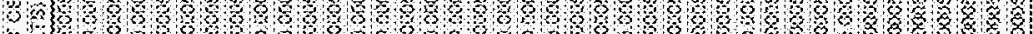
\%

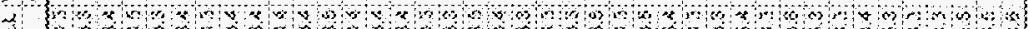

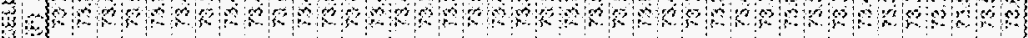

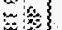
㫨

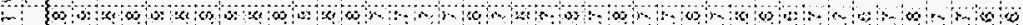

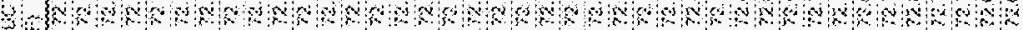
:

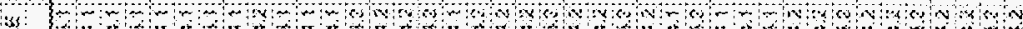
W 率:

13

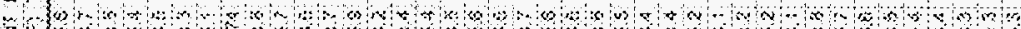
of (3) 4tas

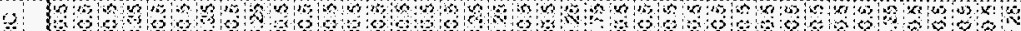

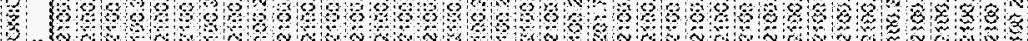

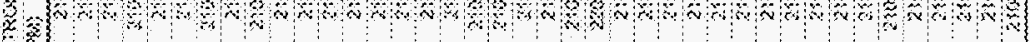
(a)

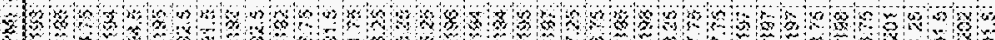

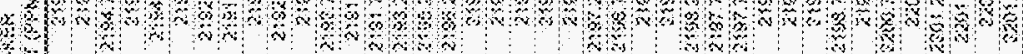

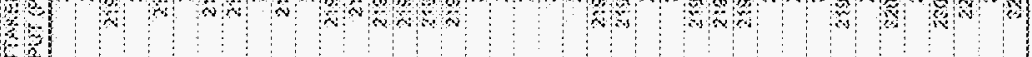
3

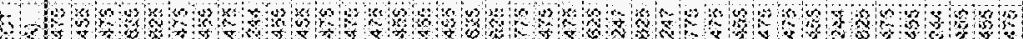

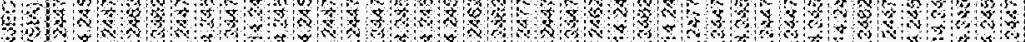

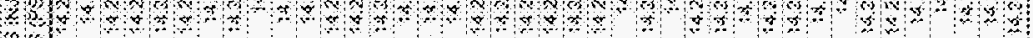
3

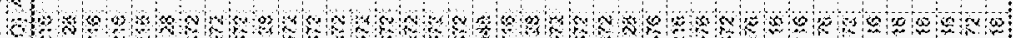

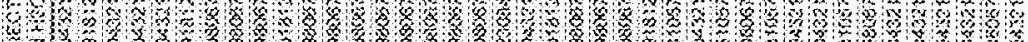

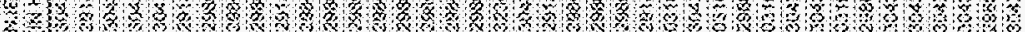

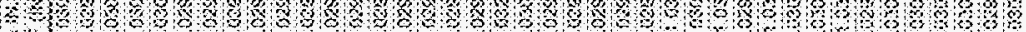

a

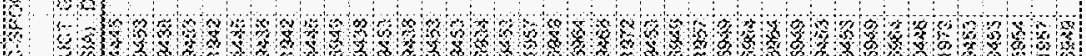

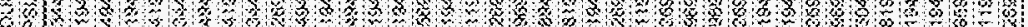
64,

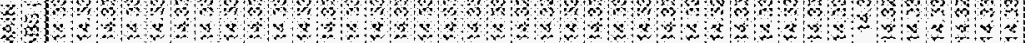
रे की

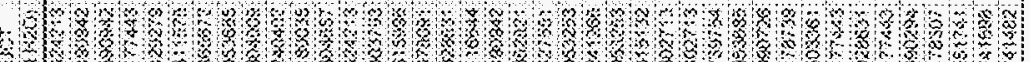

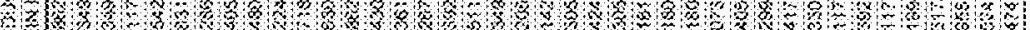

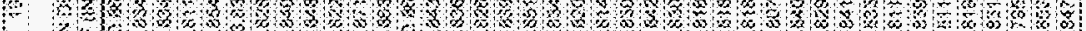
\% 4 \% W (a)

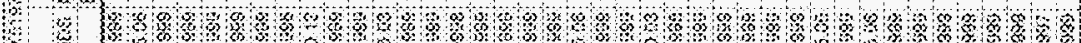

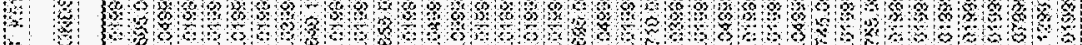

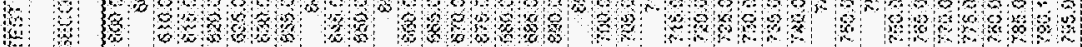




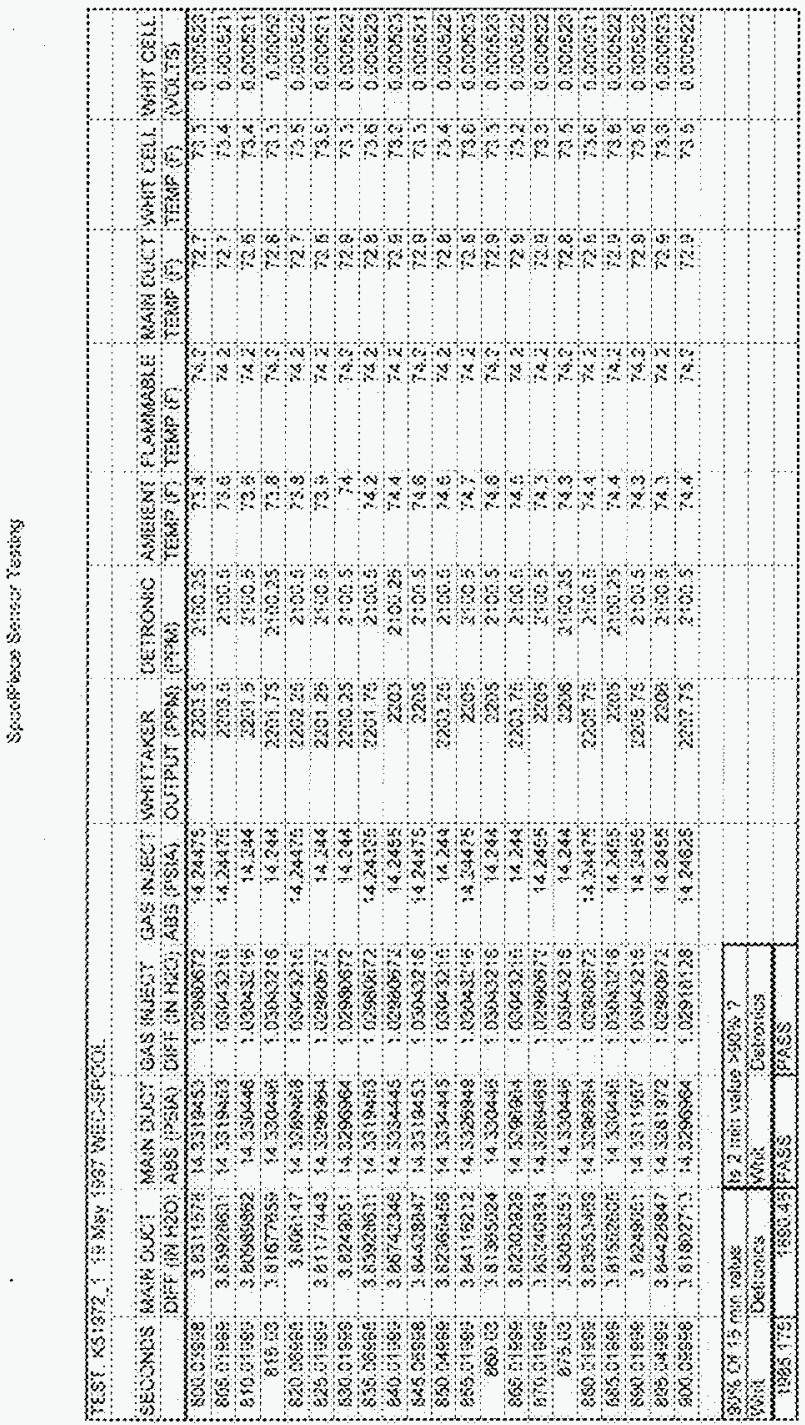




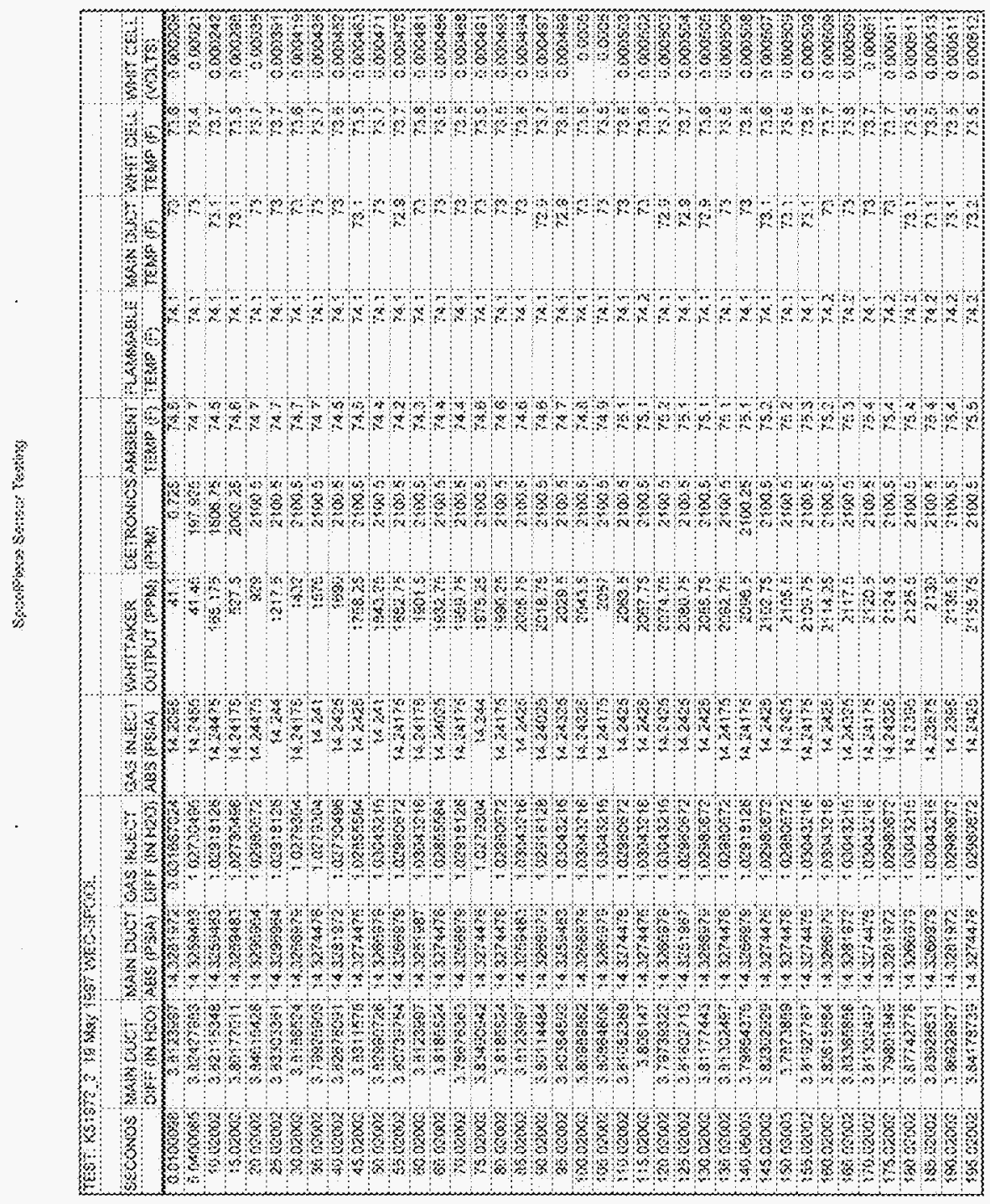




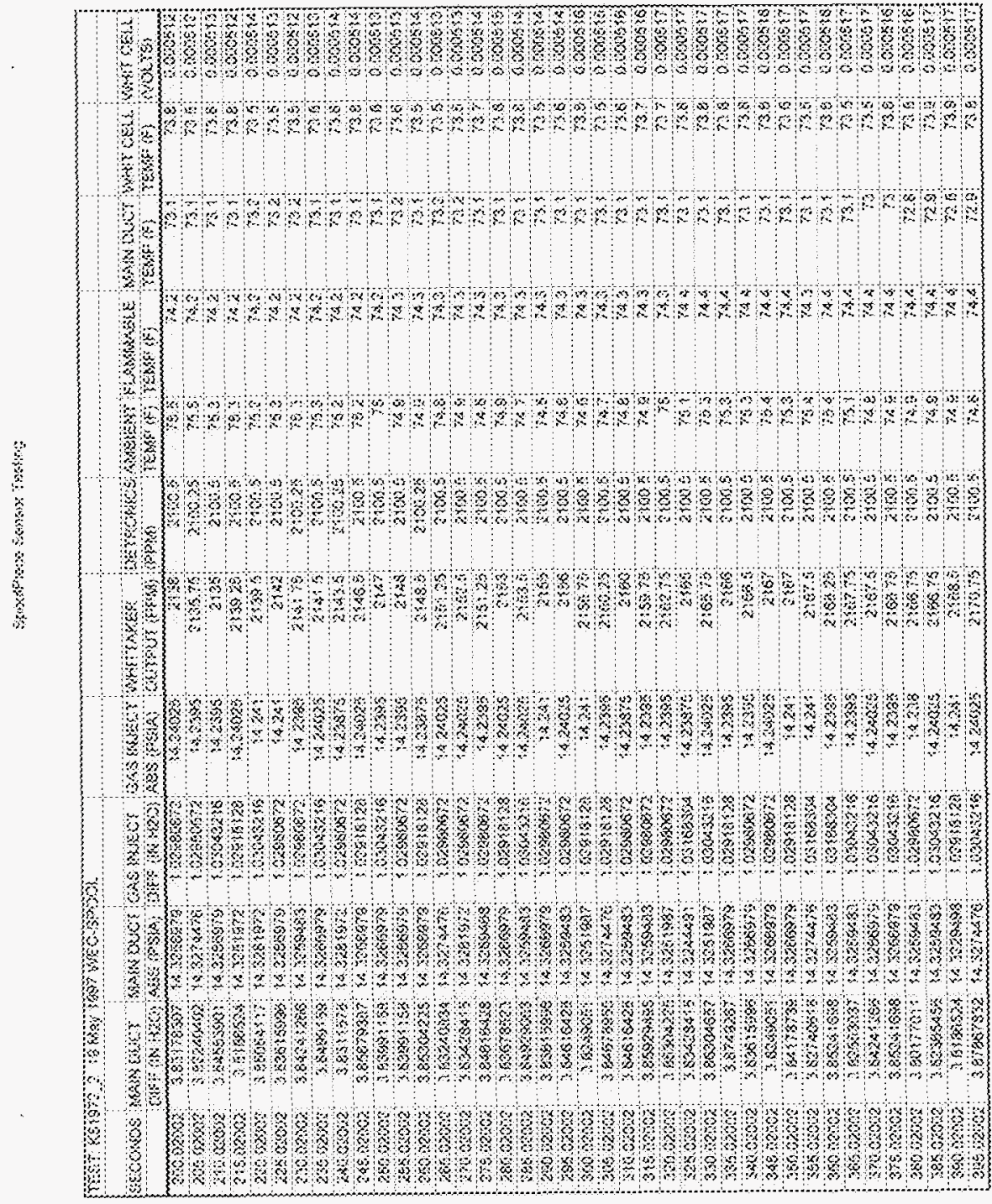


$\{34+53-142<-74\}^{3} 286$

सev, i)

(4) ?os

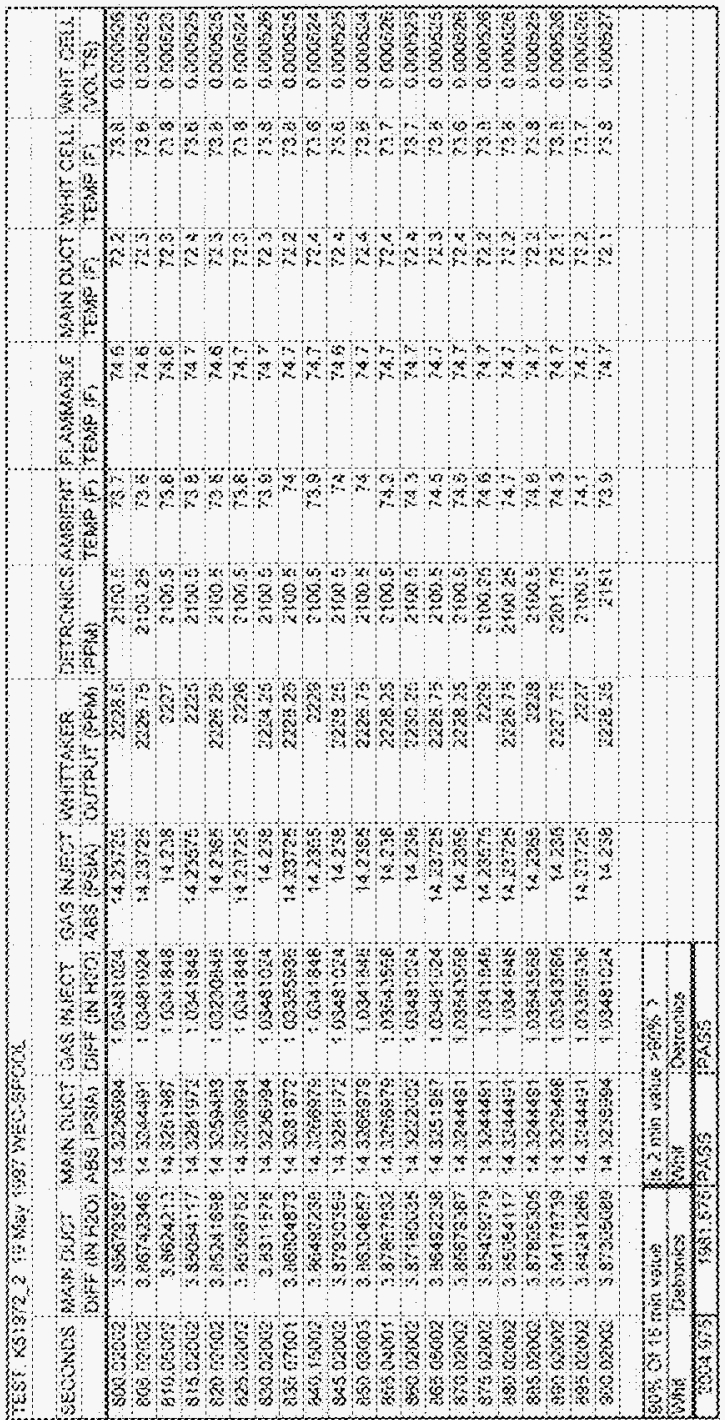




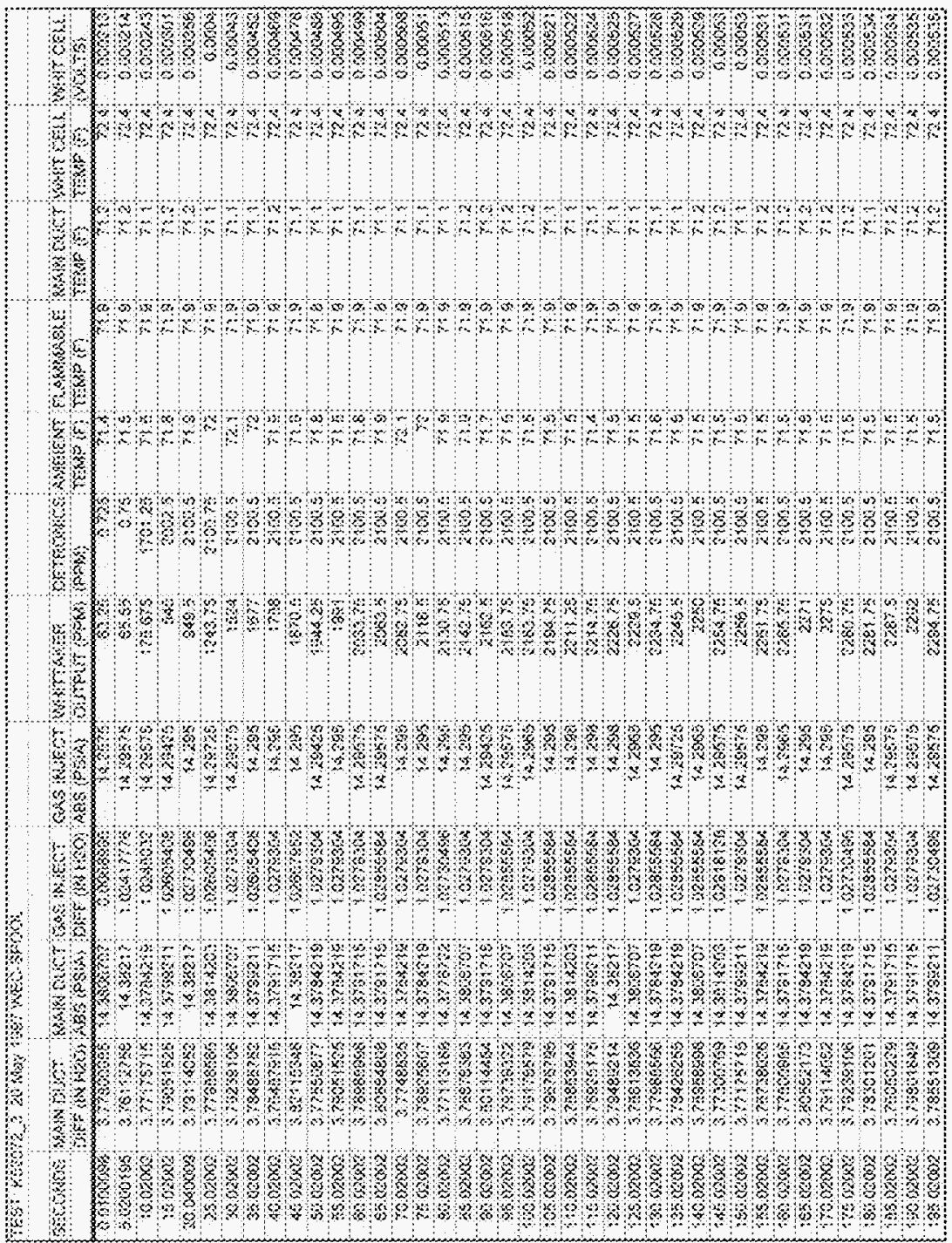




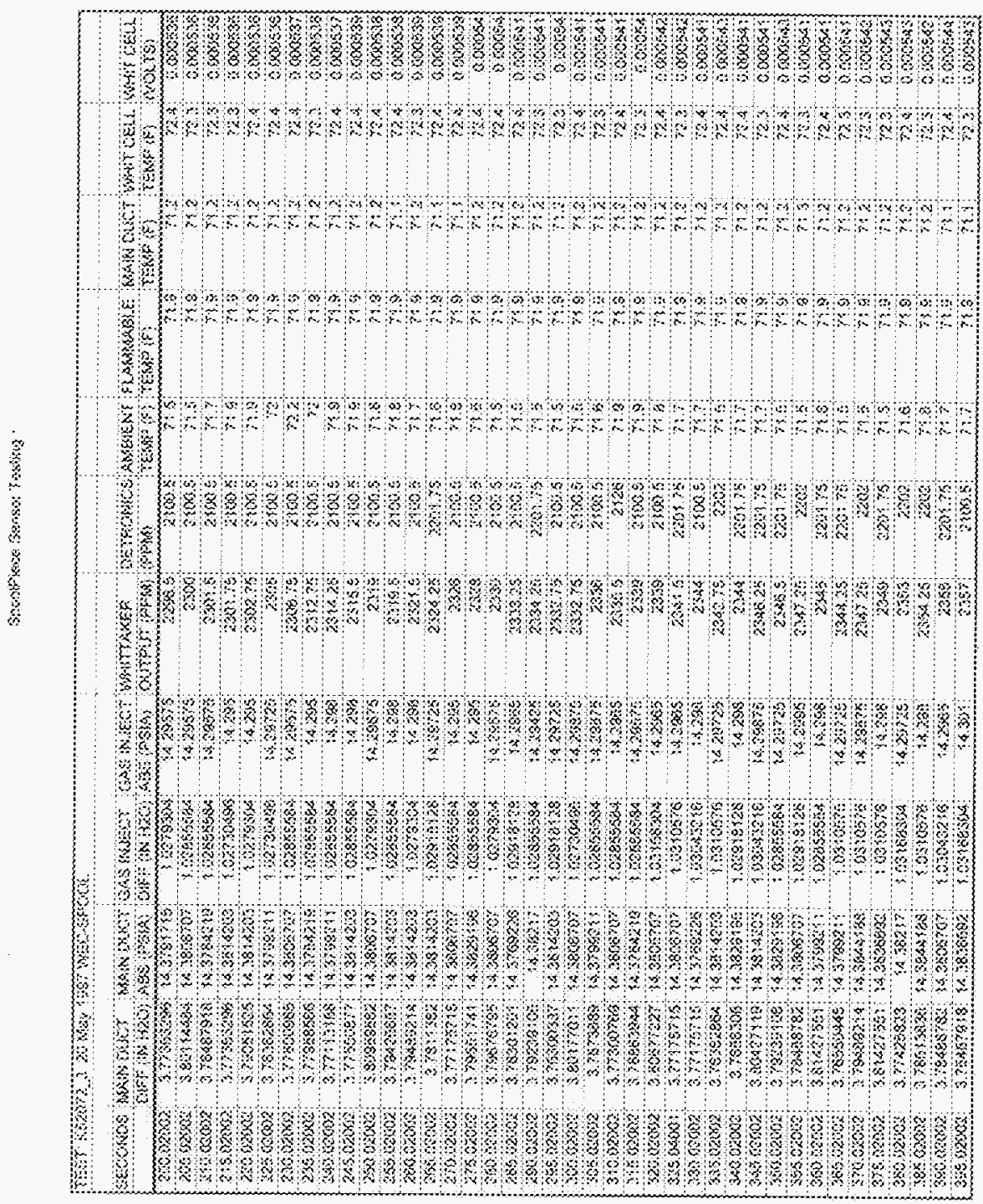




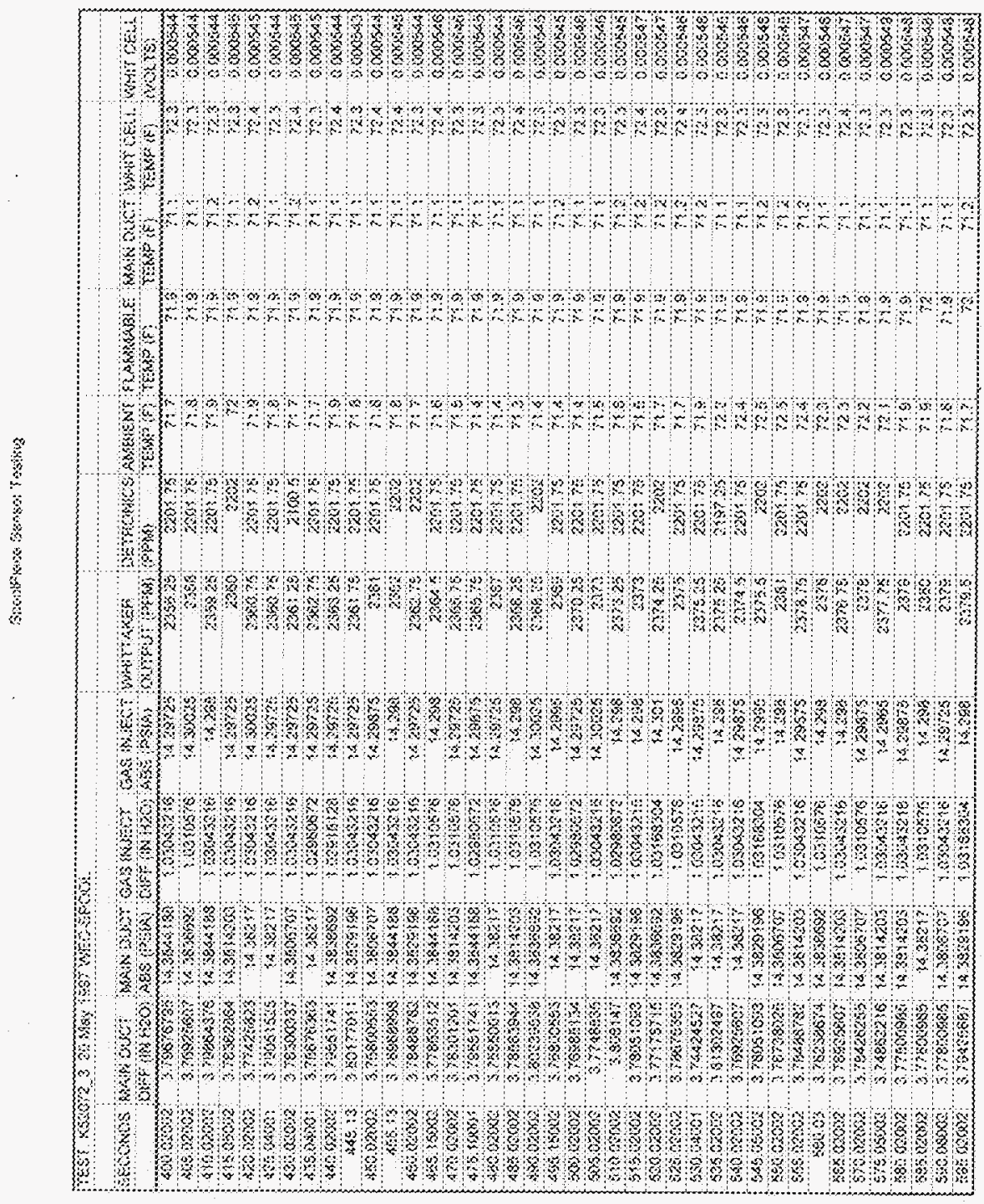




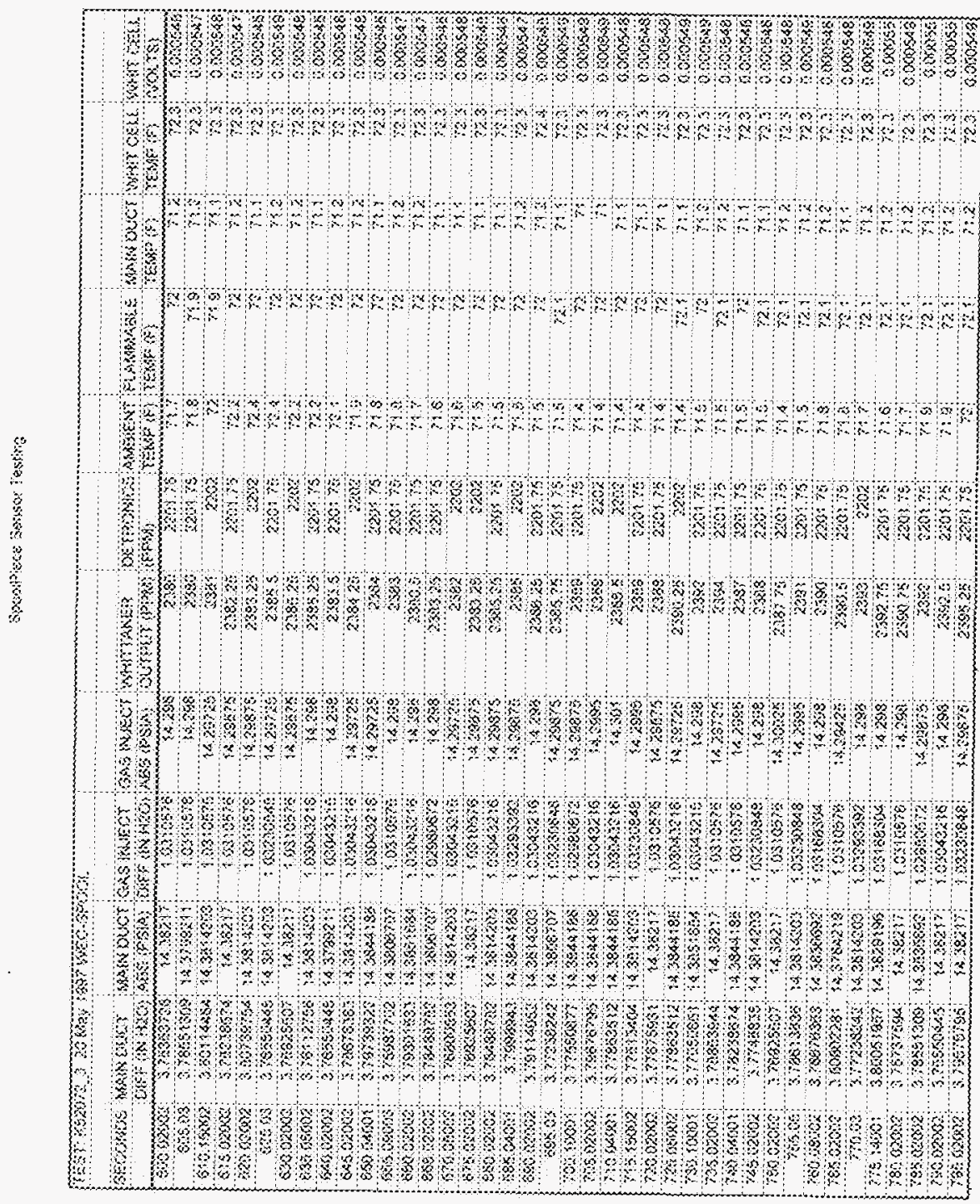




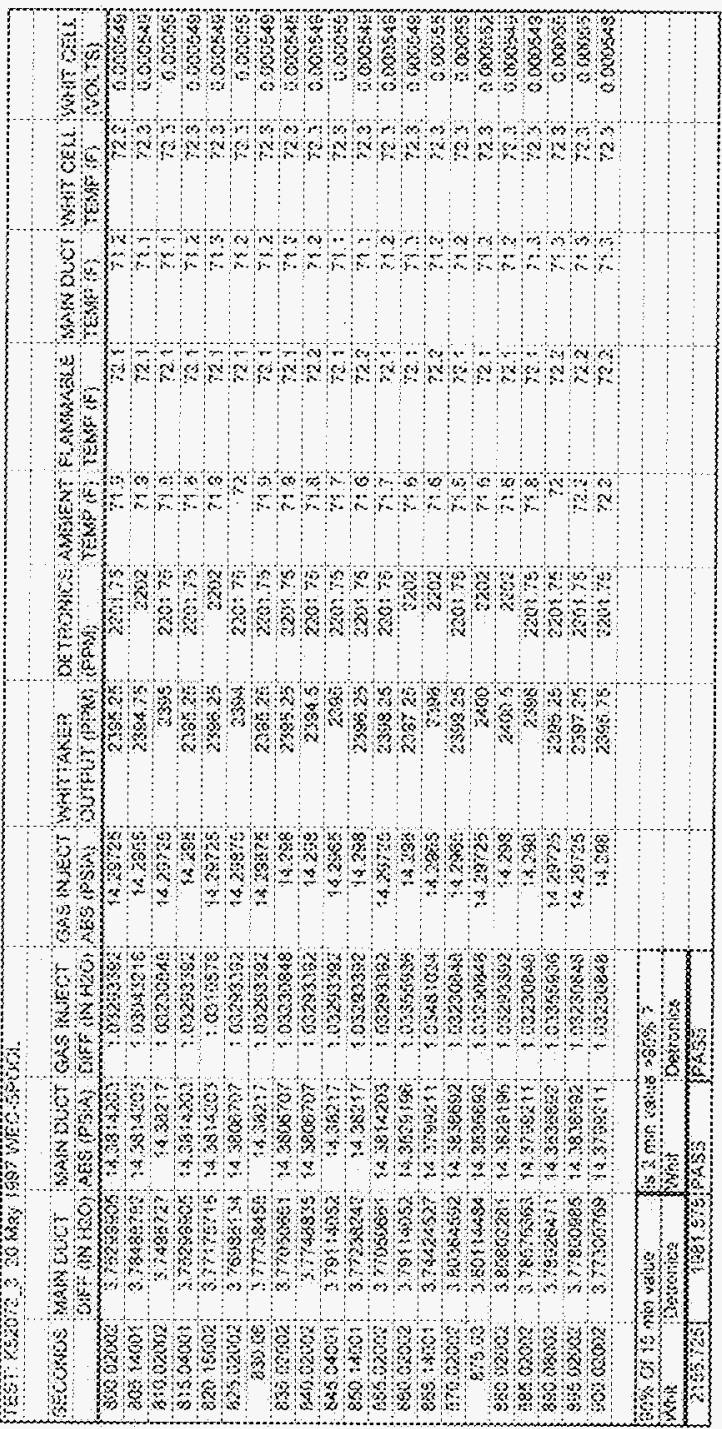




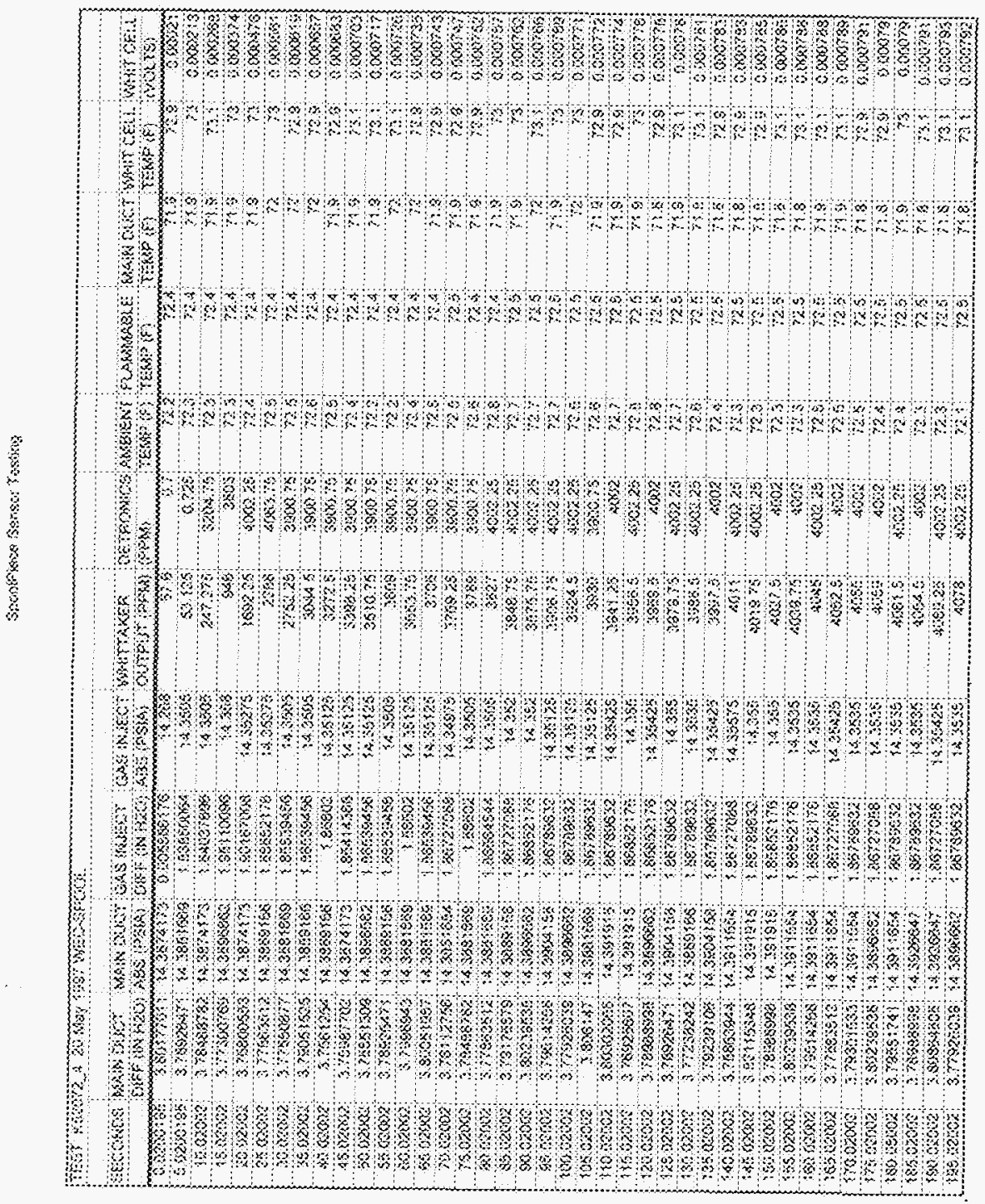




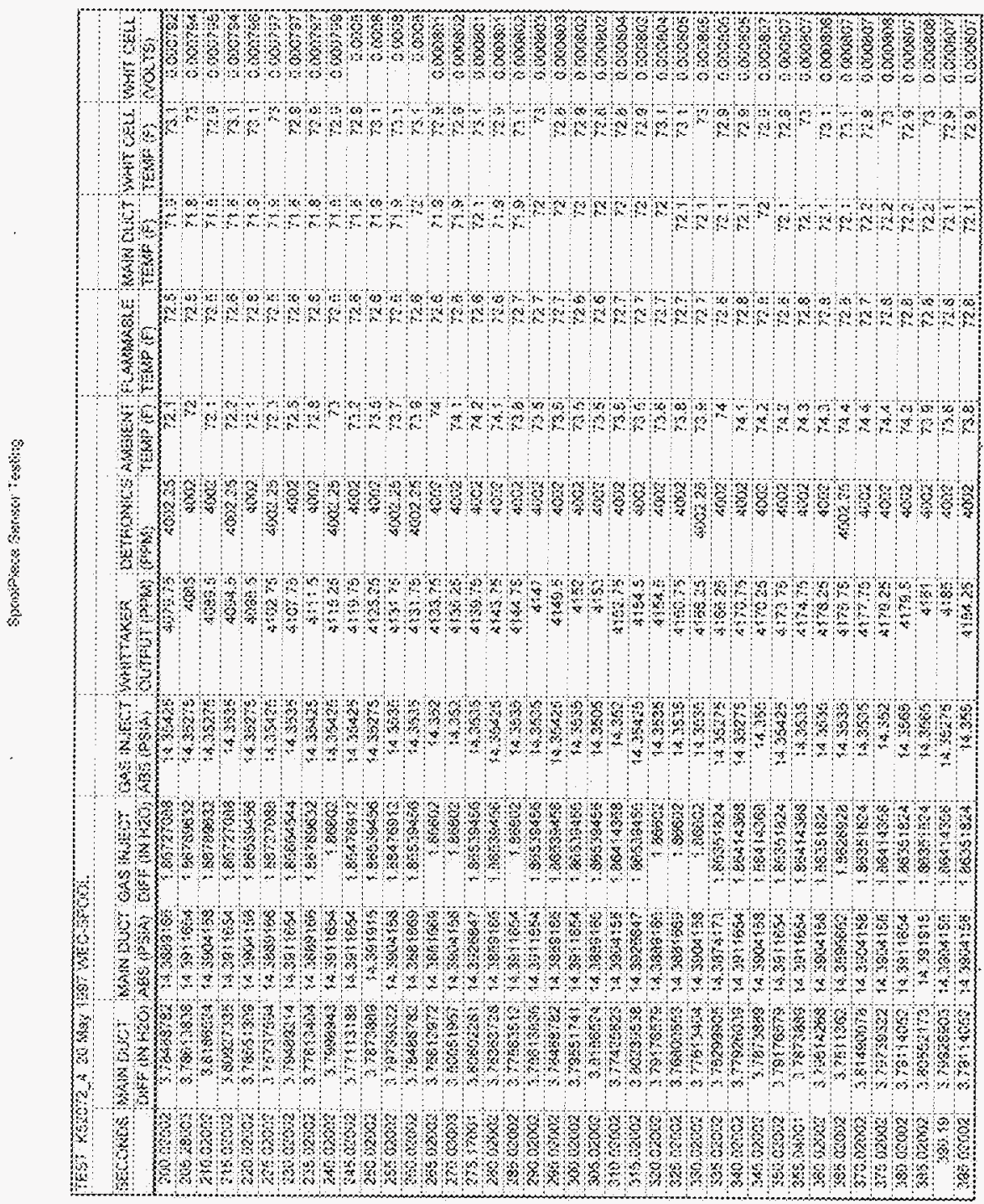




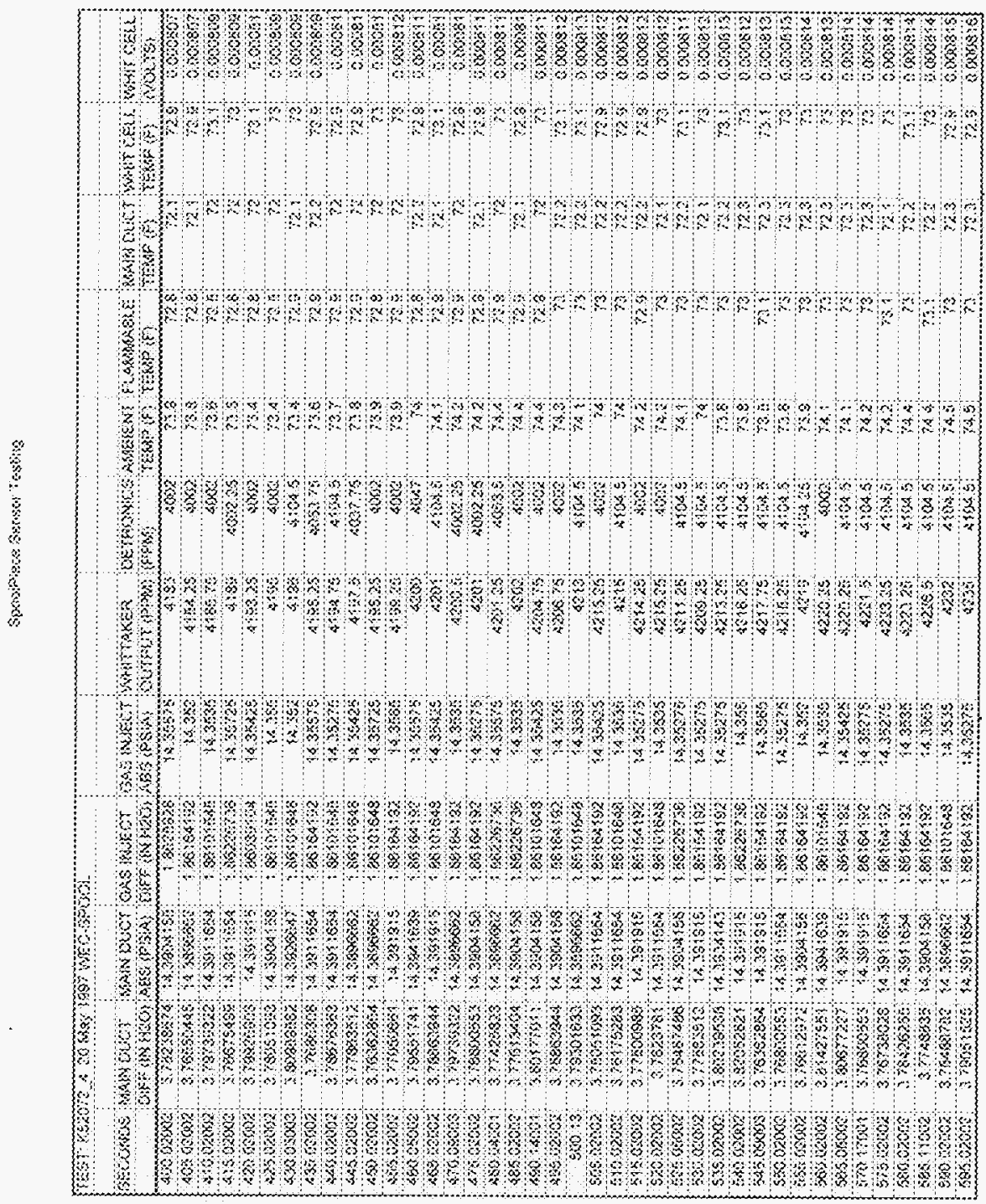




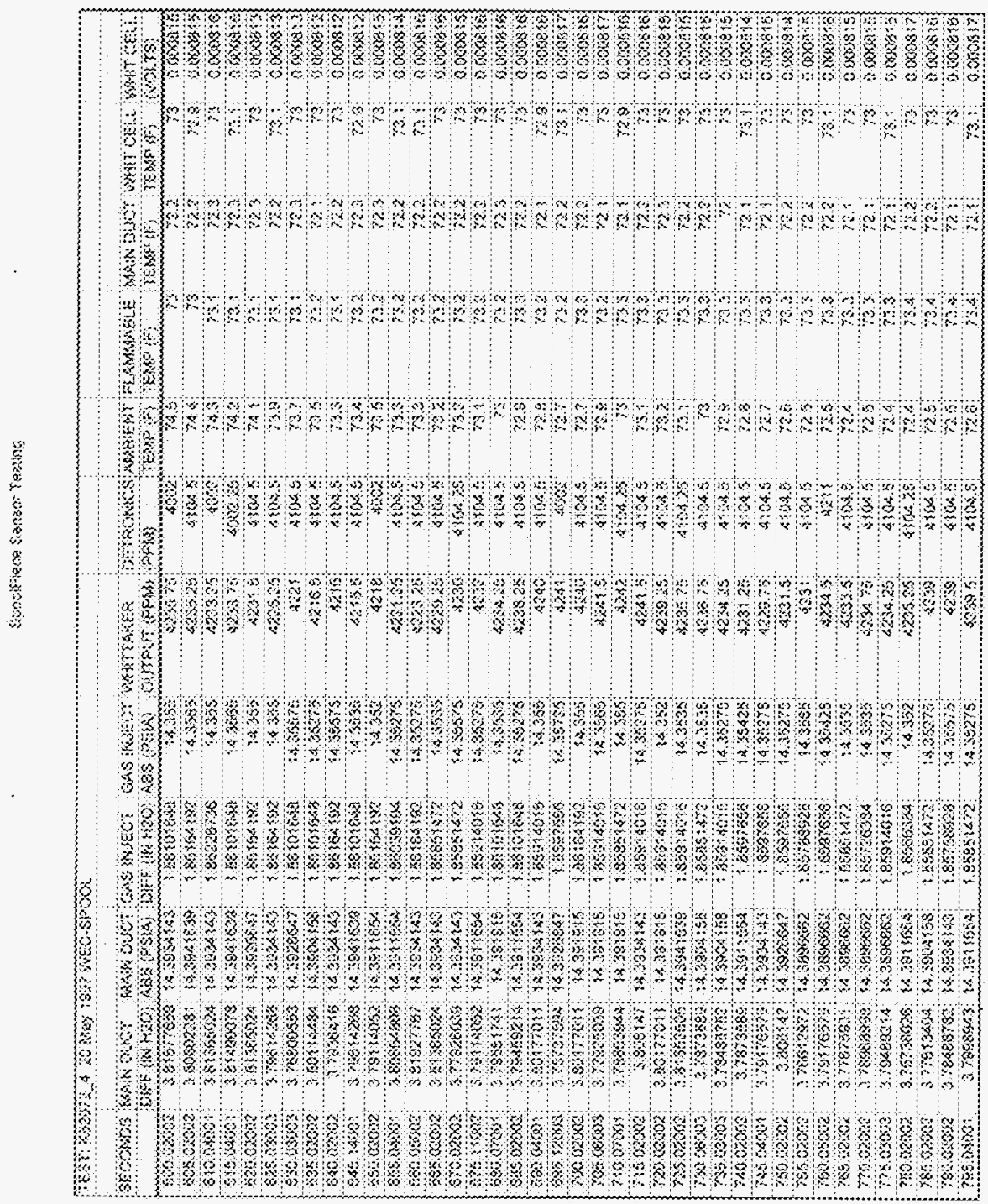




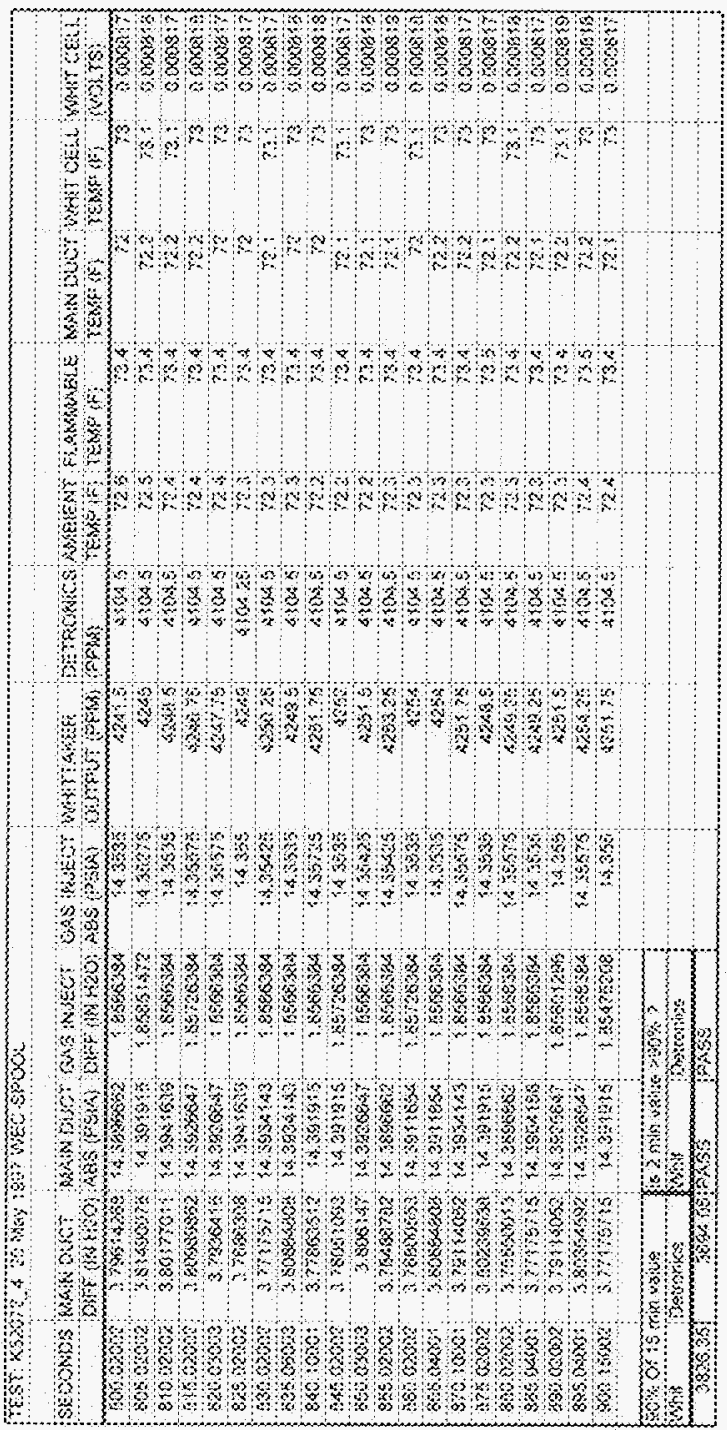




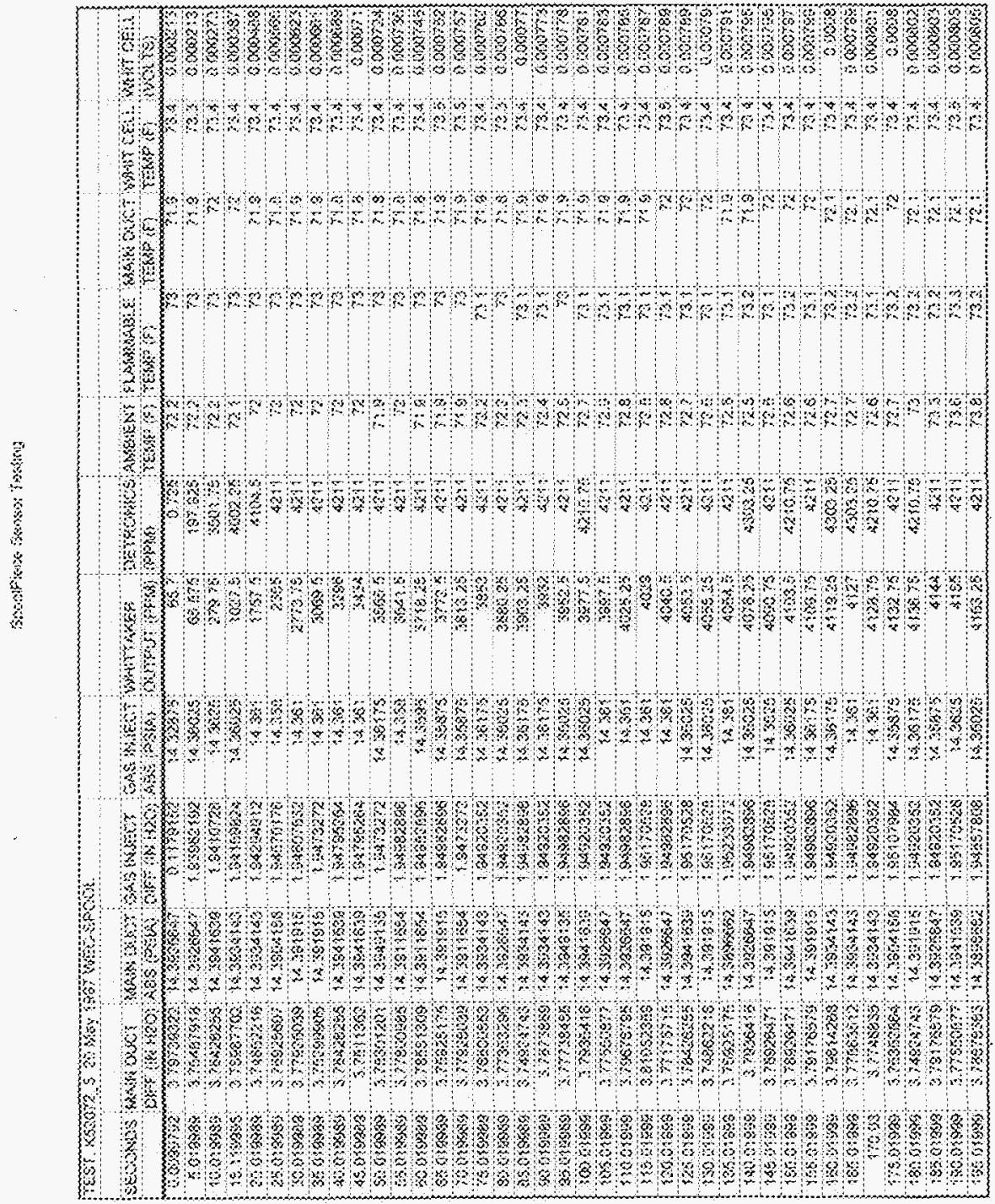




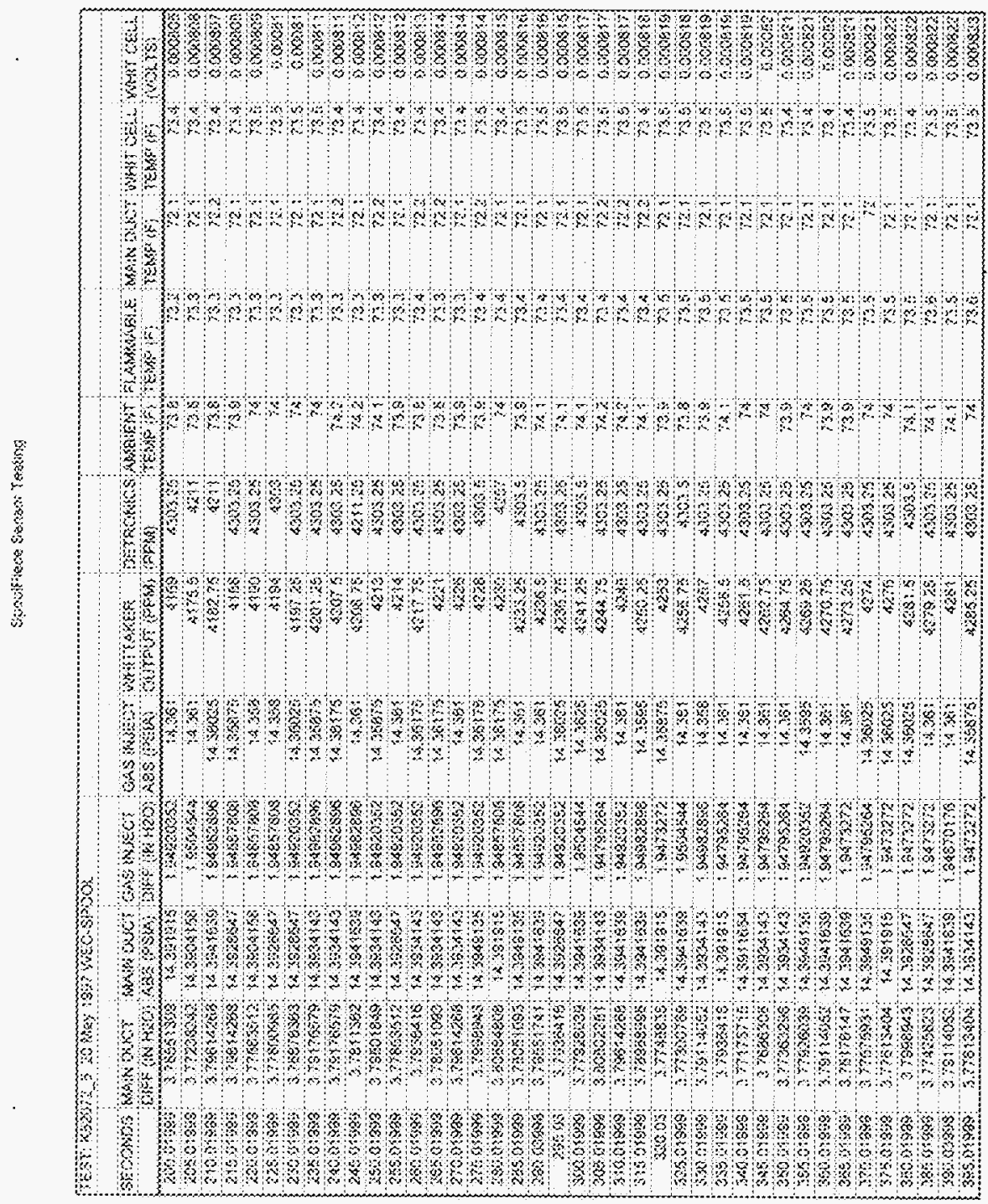




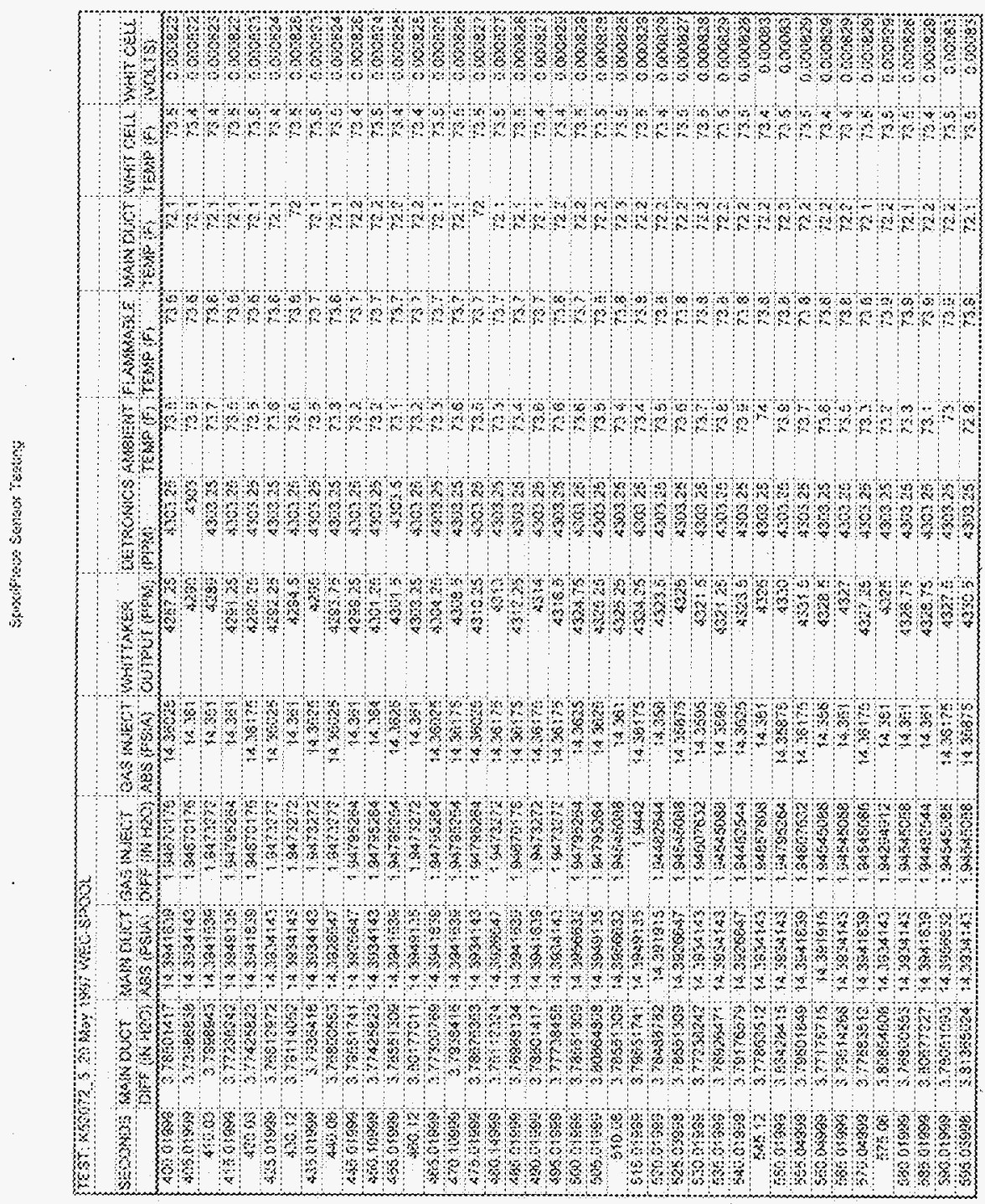




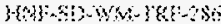

Bicy 9

pose 223

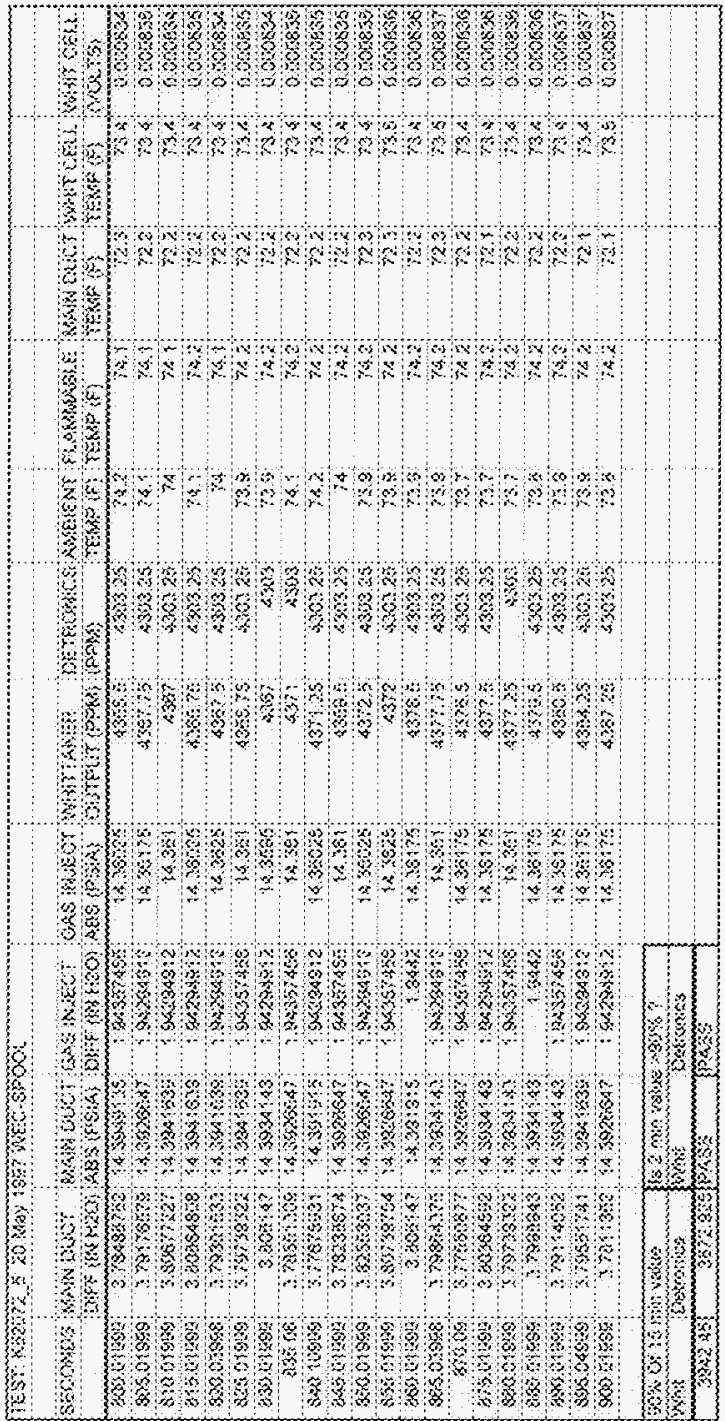




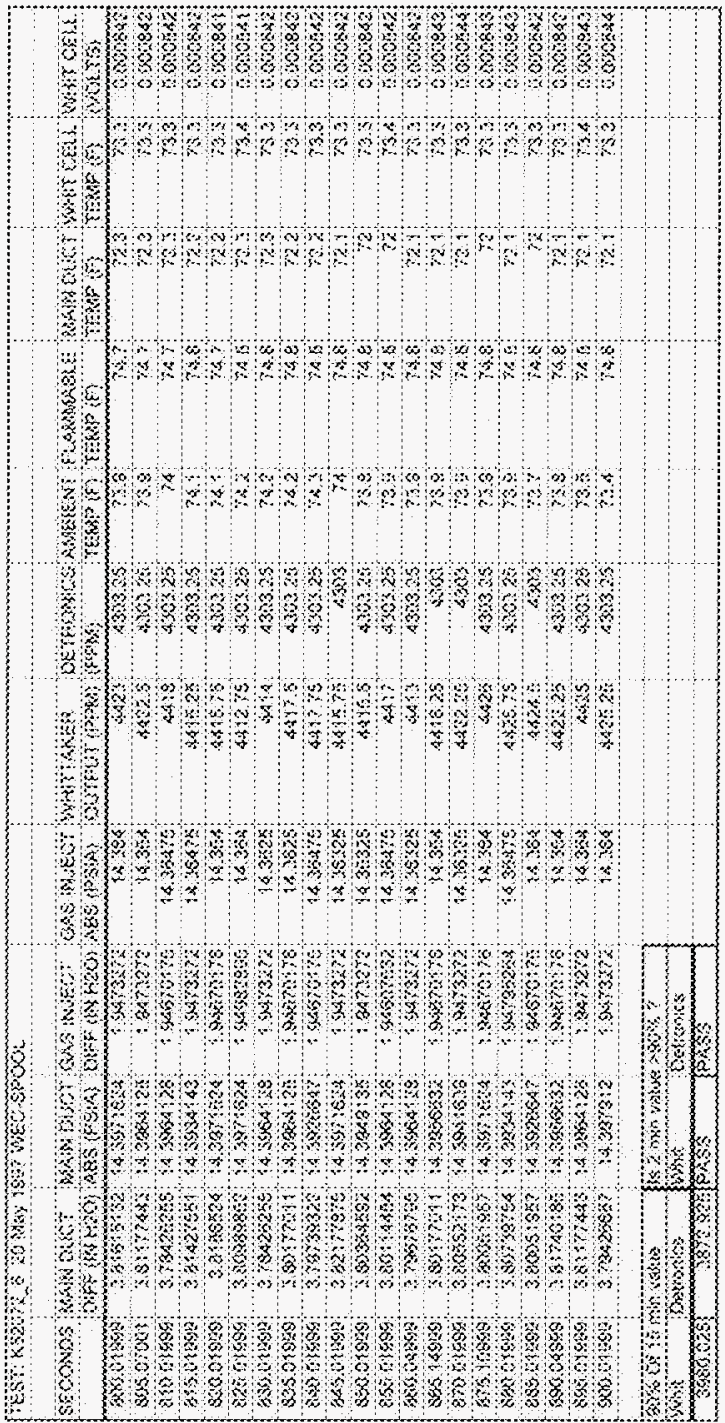




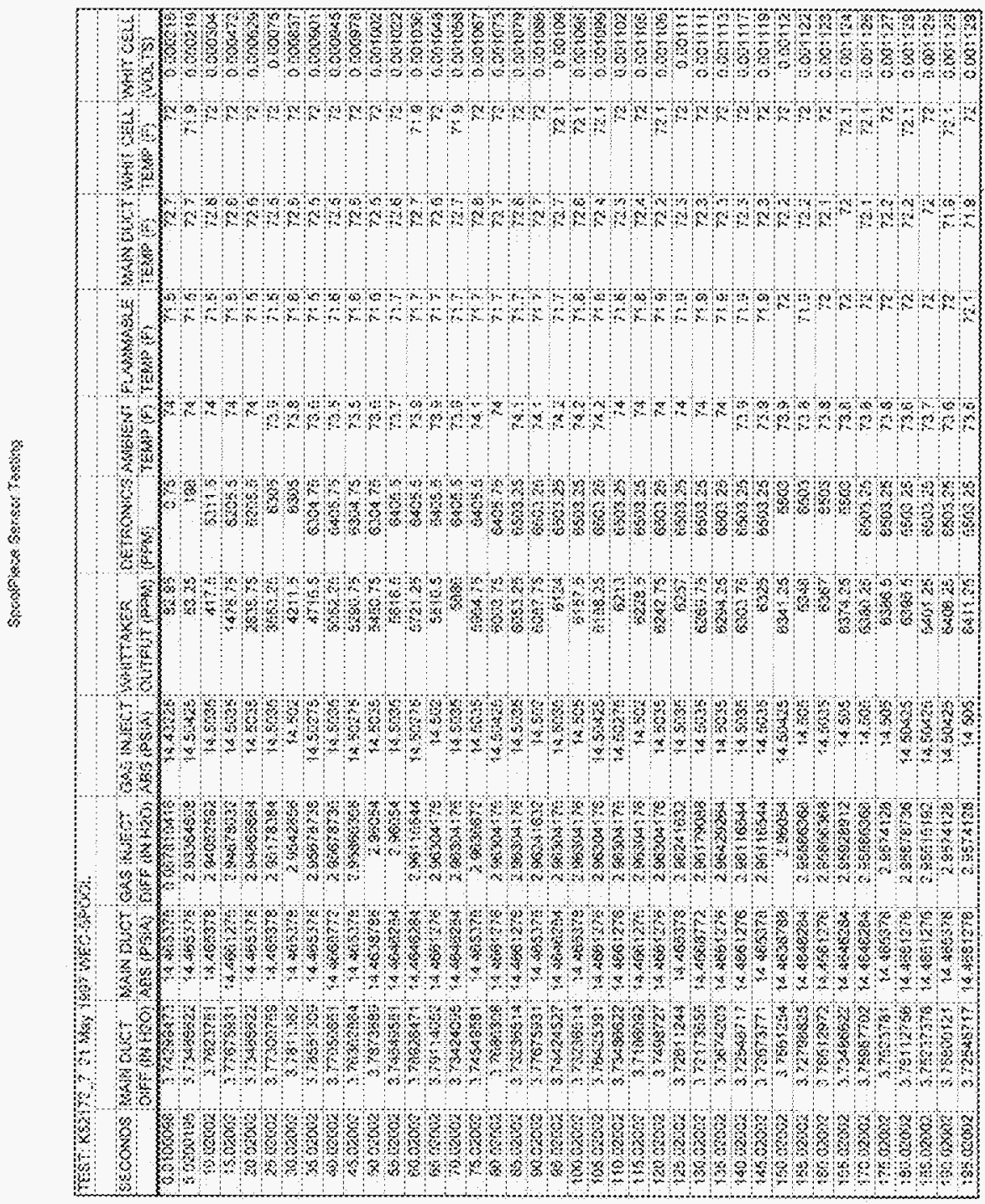




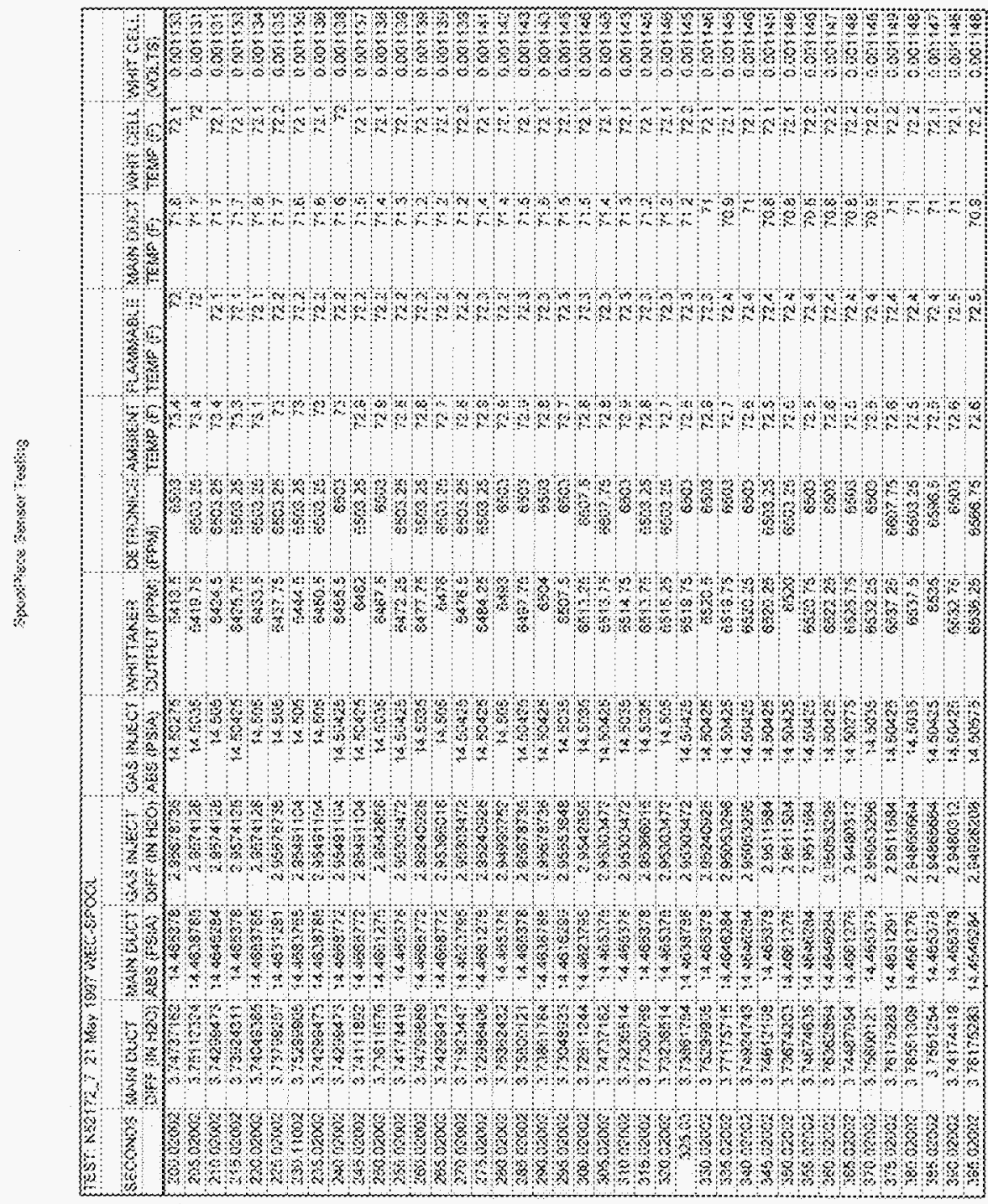




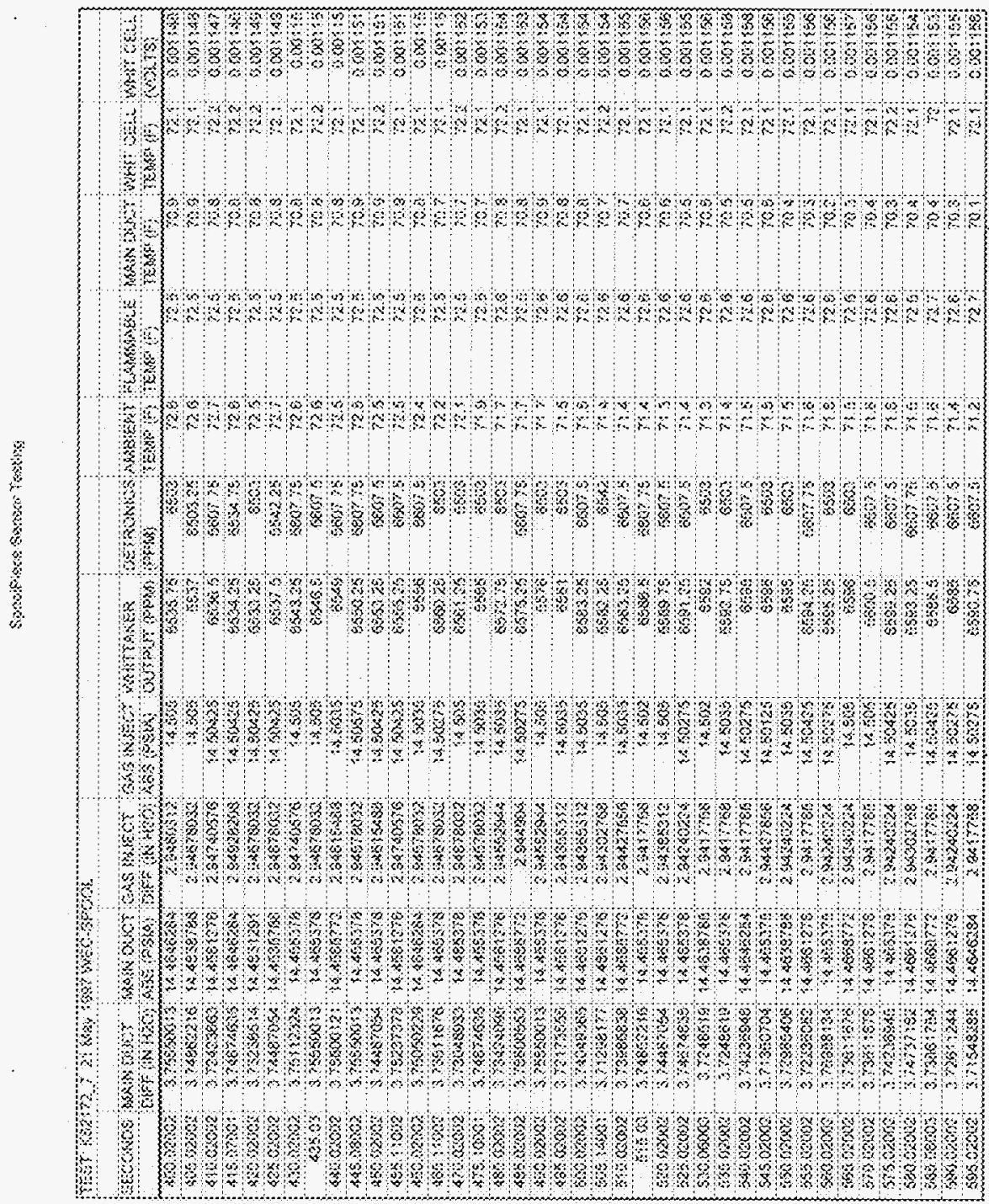




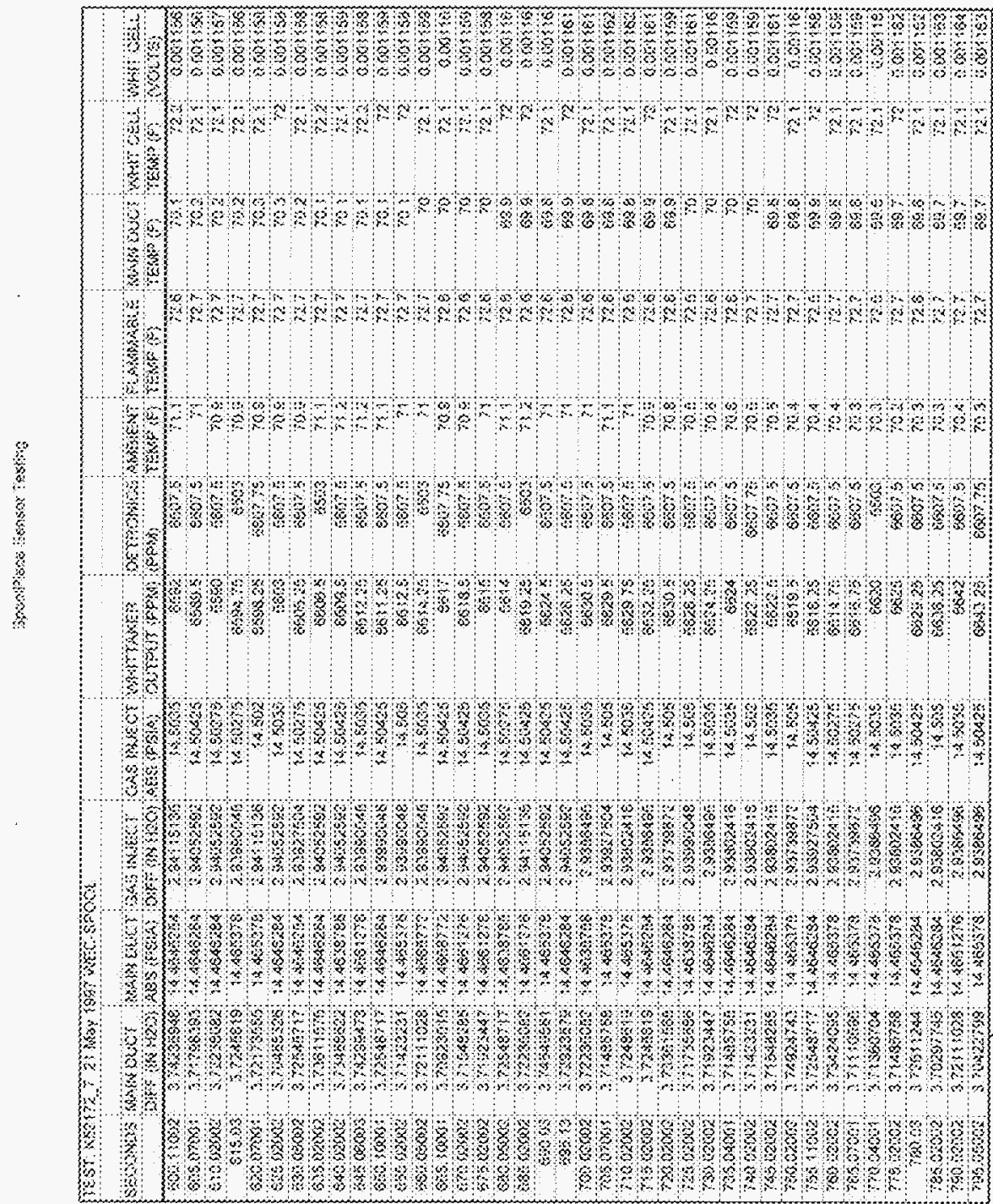




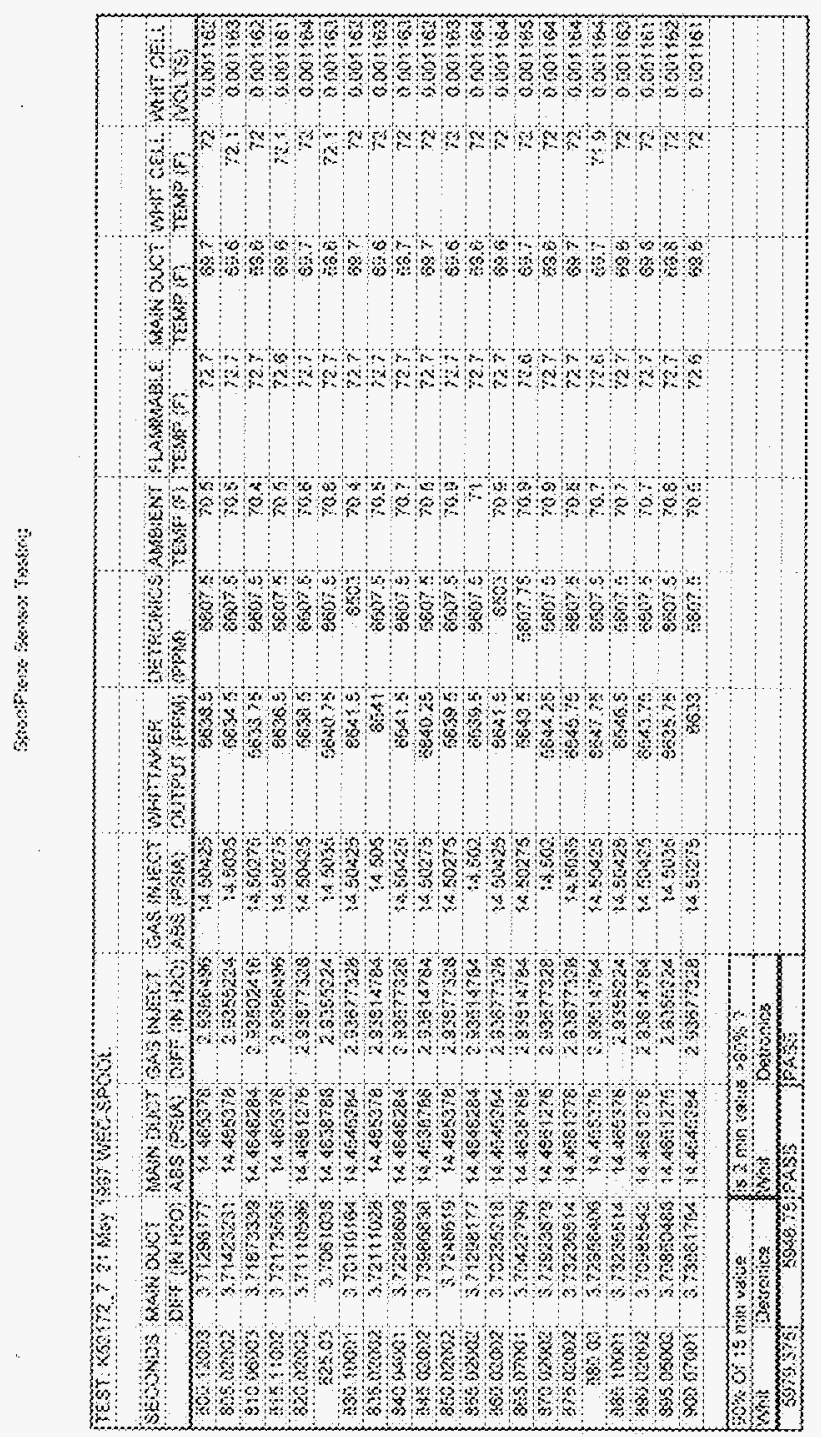




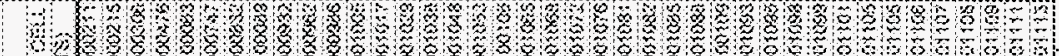

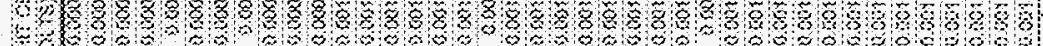

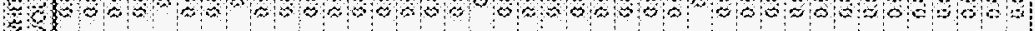
3

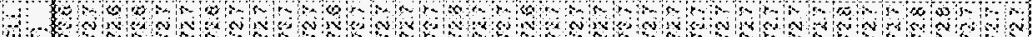

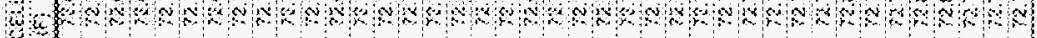
涴 (3)

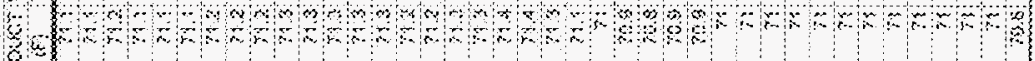
\%

3

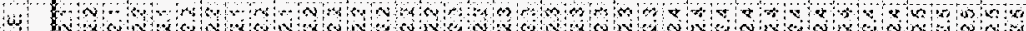

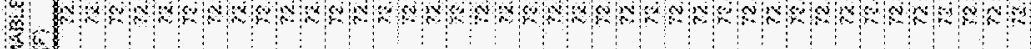
帘罂 (3)

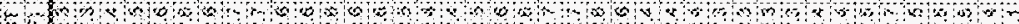

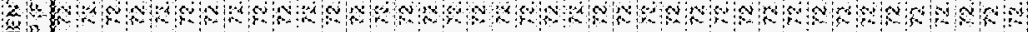
㫨

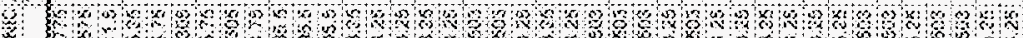
कo a

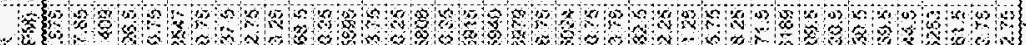
w

流

澏

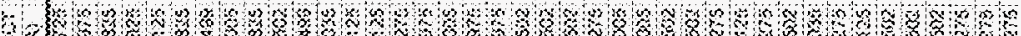

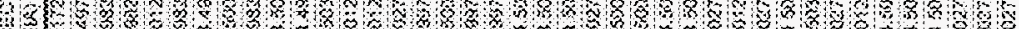
zसf o

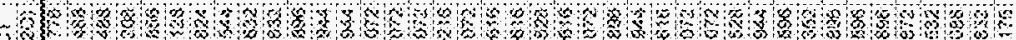
3. 4 \%

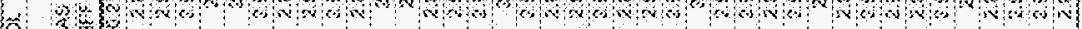

\$o $3 \mathrm{~s}$

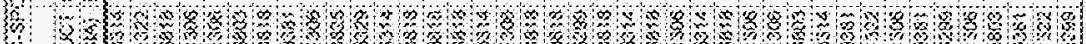

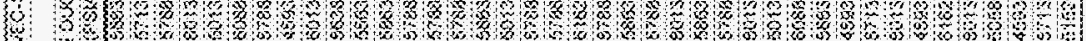

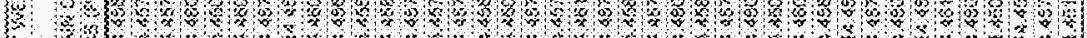

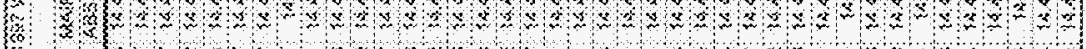

- कW

E 2 कs

I

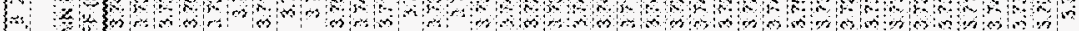

\&

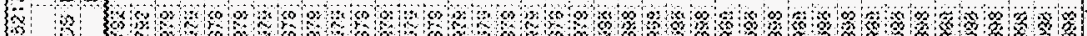
N 3 का

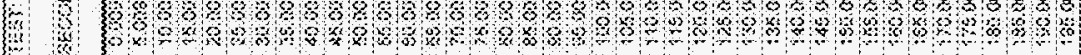




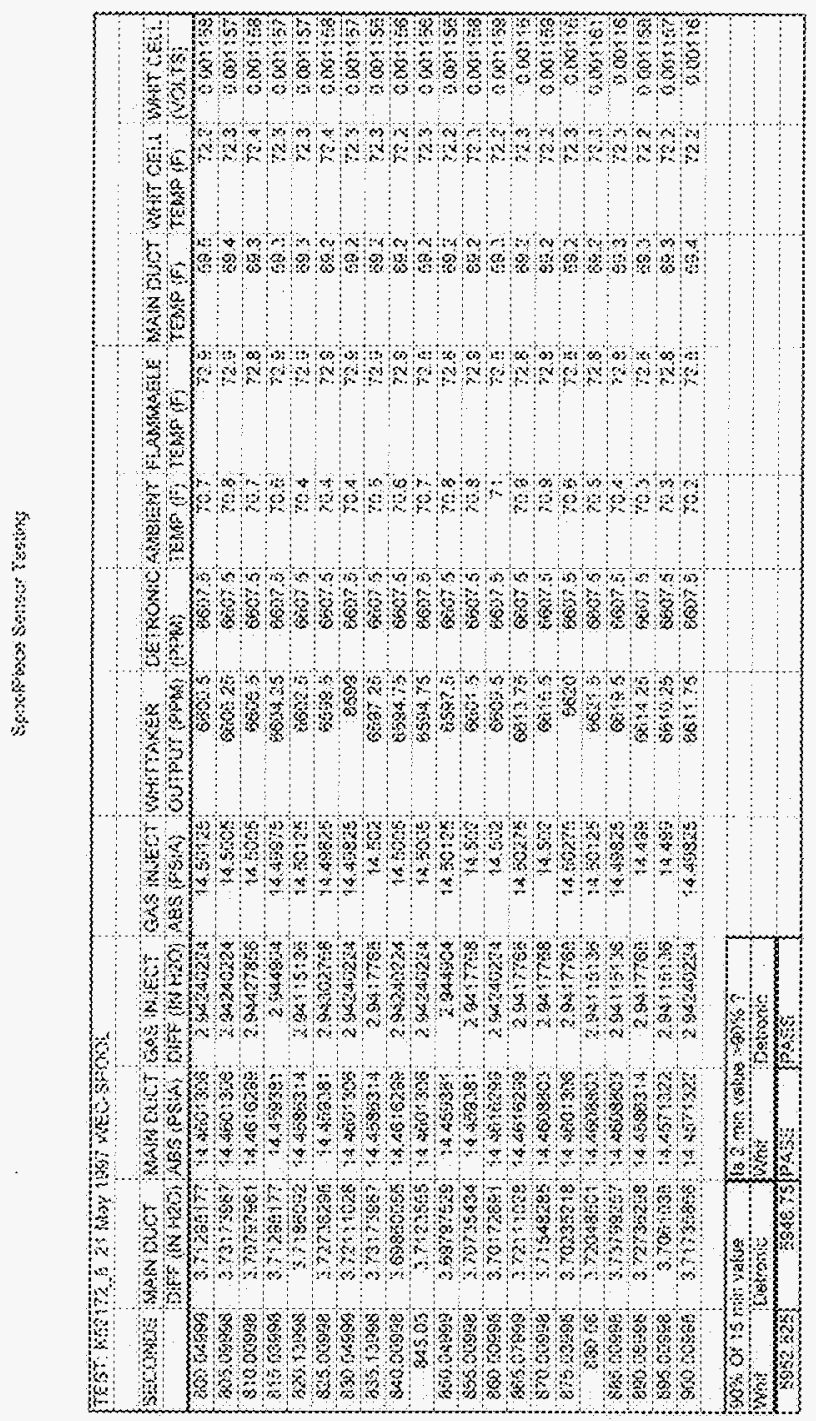




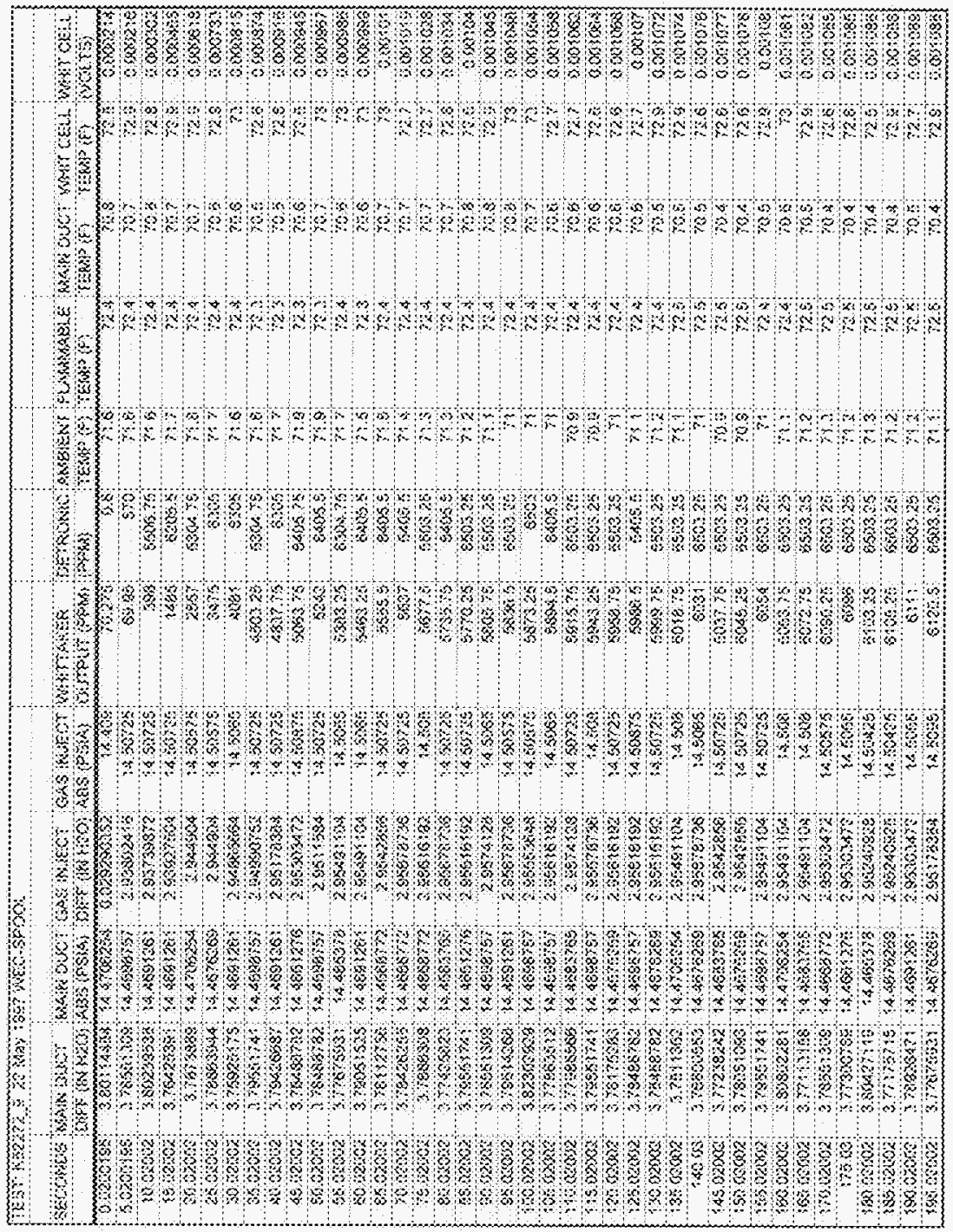


a

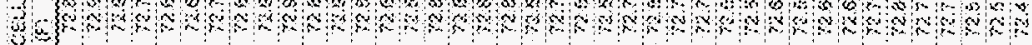

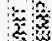

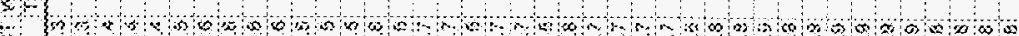

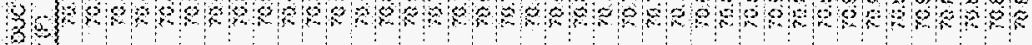

演

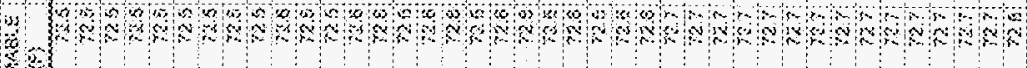

in

-

3

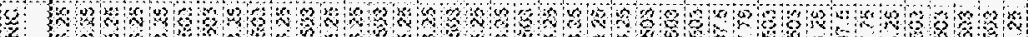
«स\% 㱐

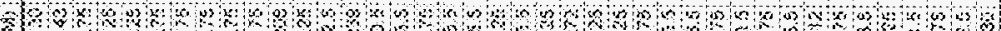
स\%

8

$\ldots$

漠:

m

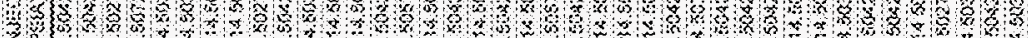

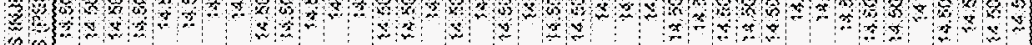

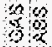

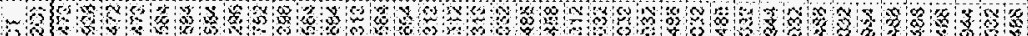
of 3 \%

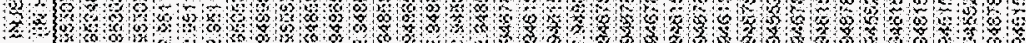

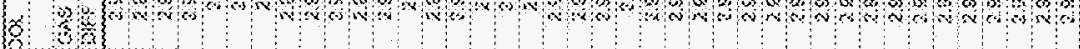

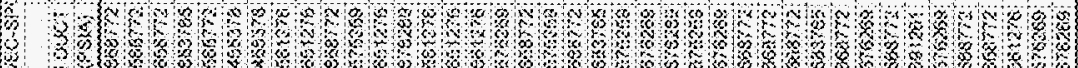

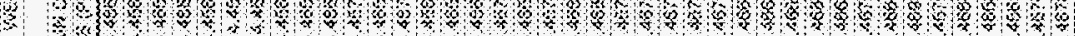

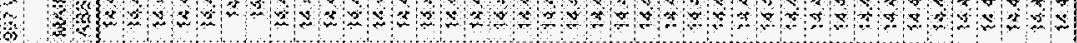

का

作

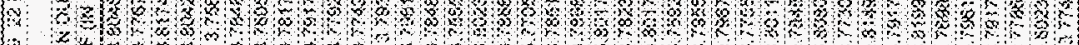

y

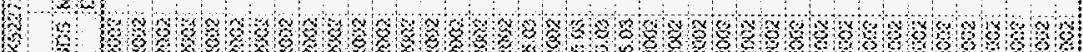

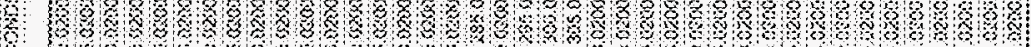

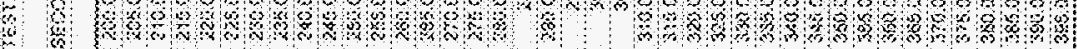




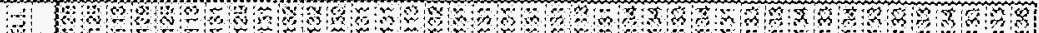

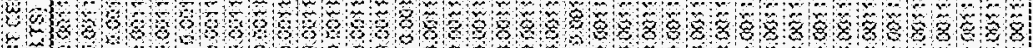
की 21

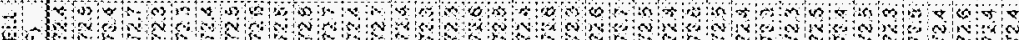

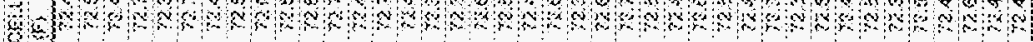
ia

3

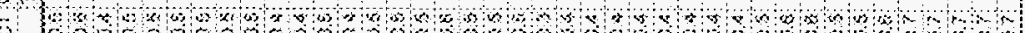

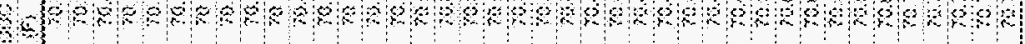
$2 a$

3

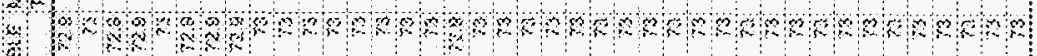
8

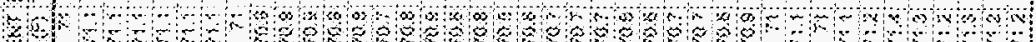

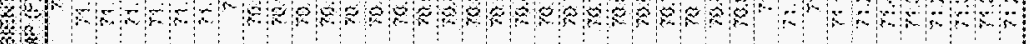

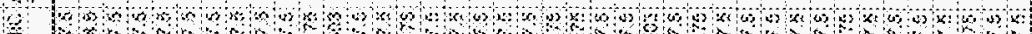

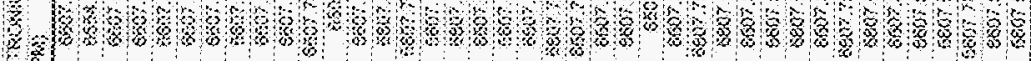
(3)

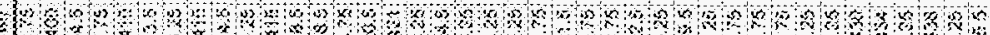

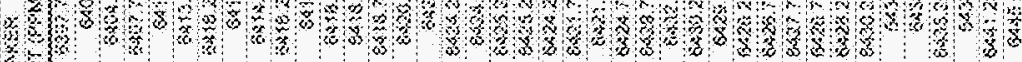
te

3

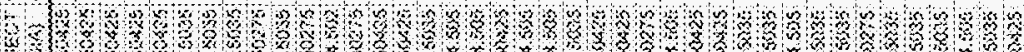

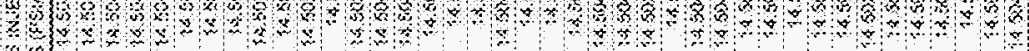
wa

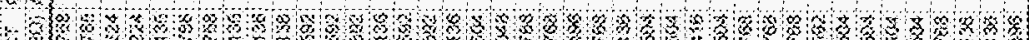

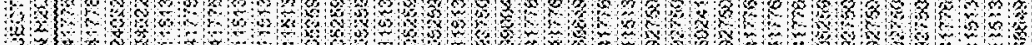

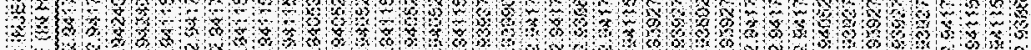
0 \%

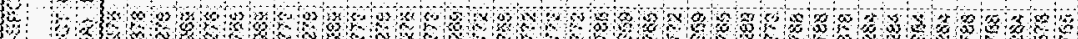

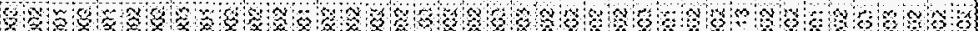

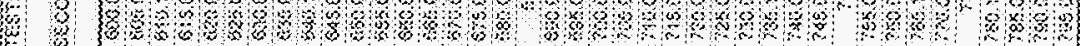




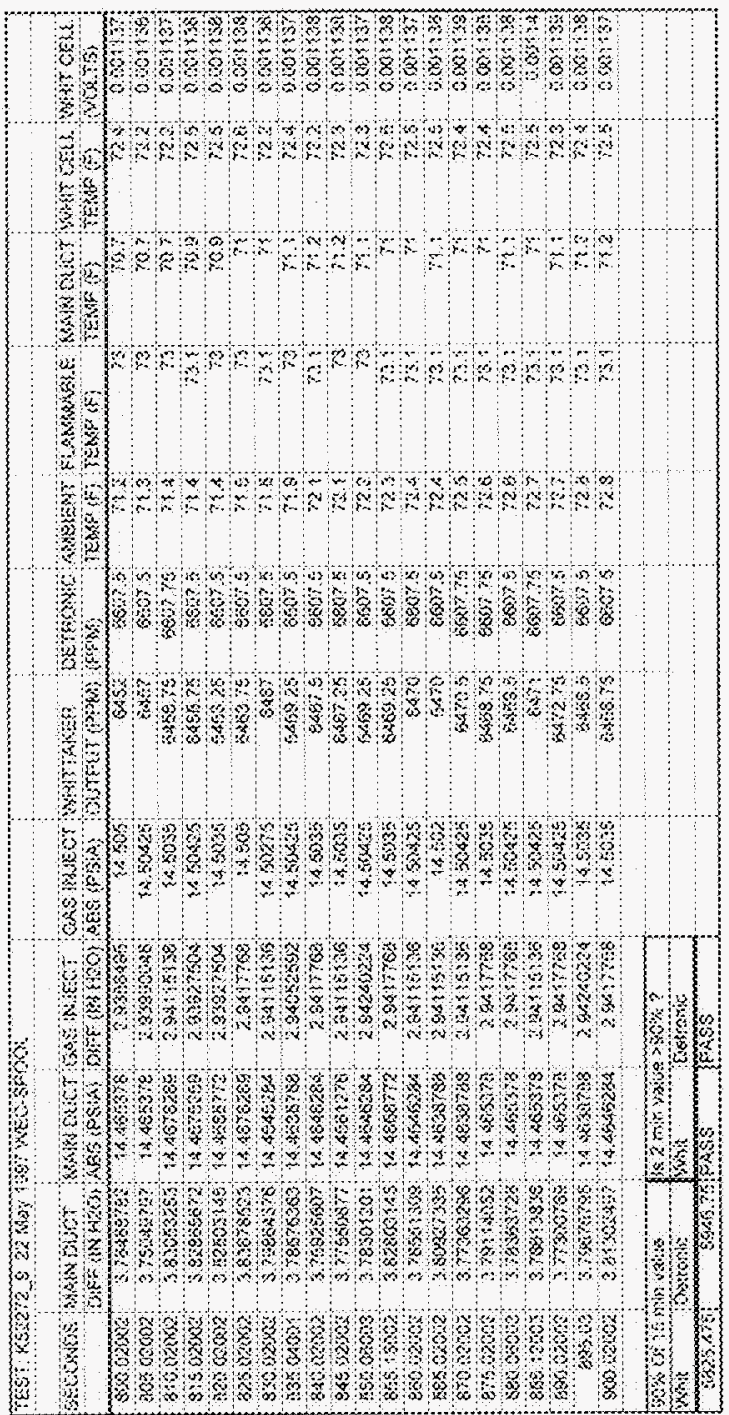




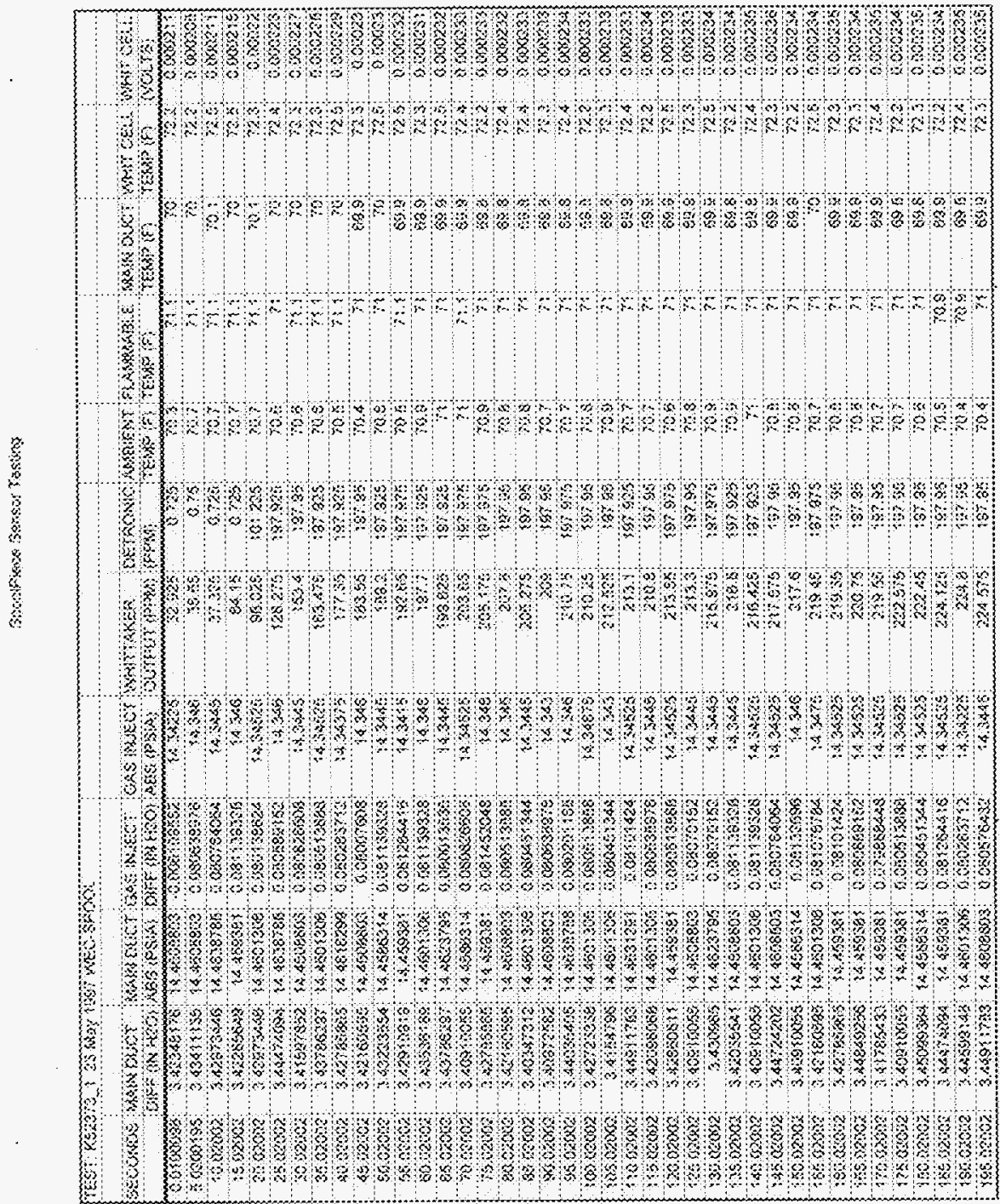




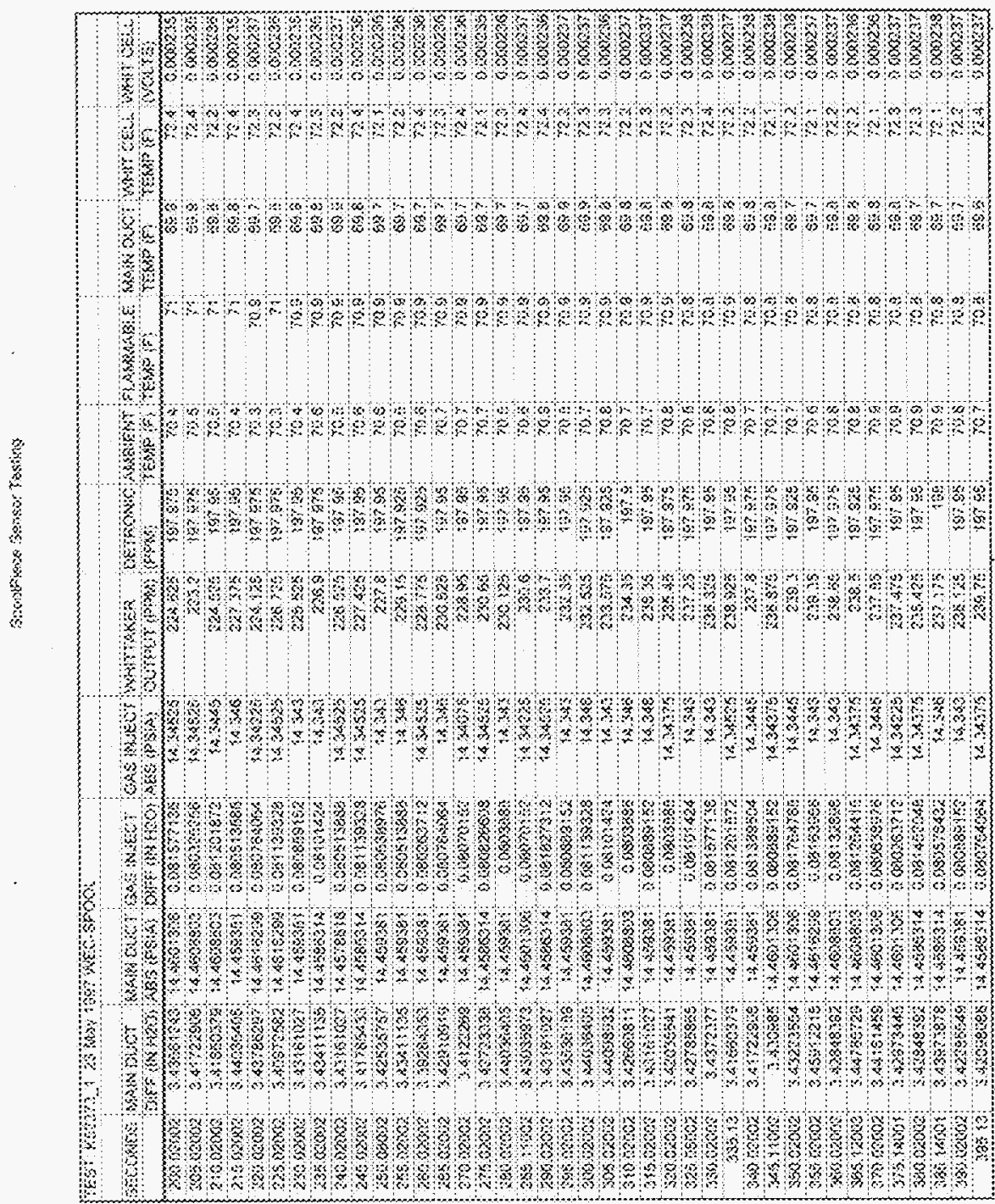




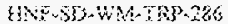

kevis

mase 14

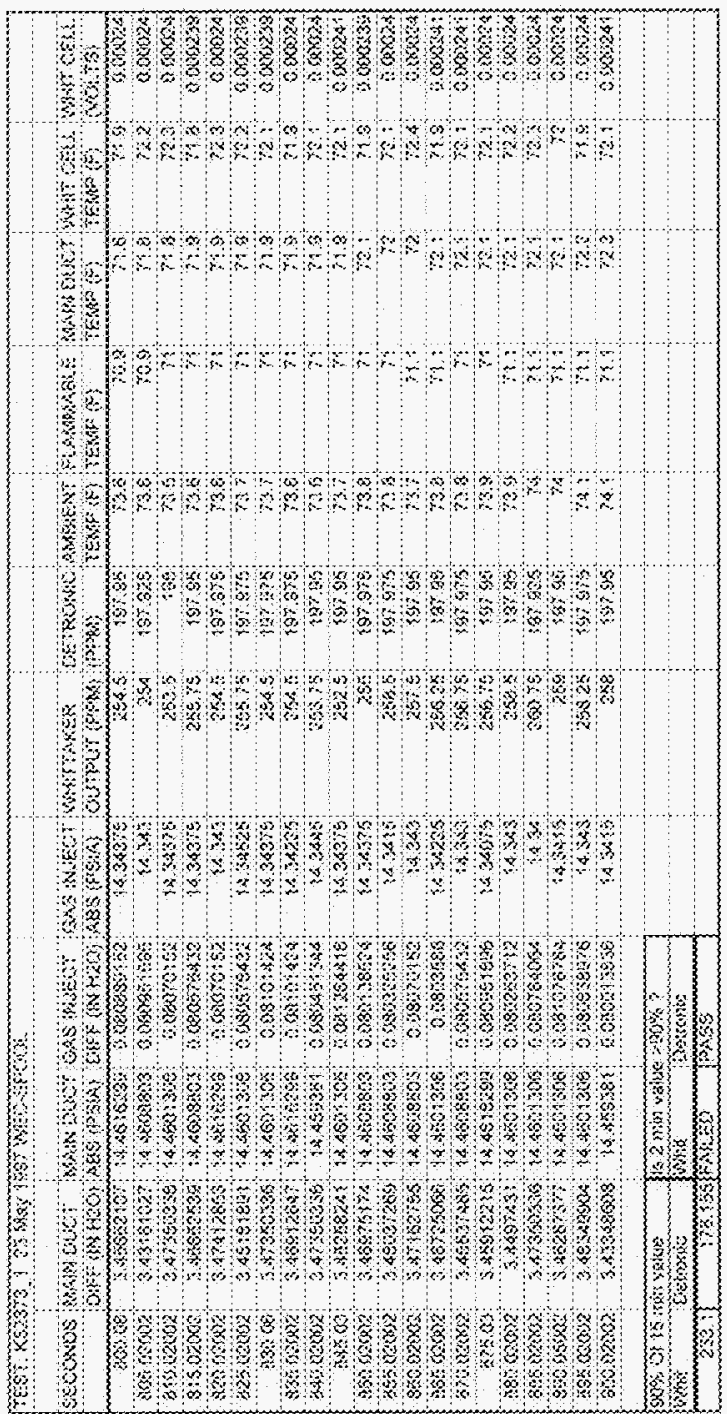




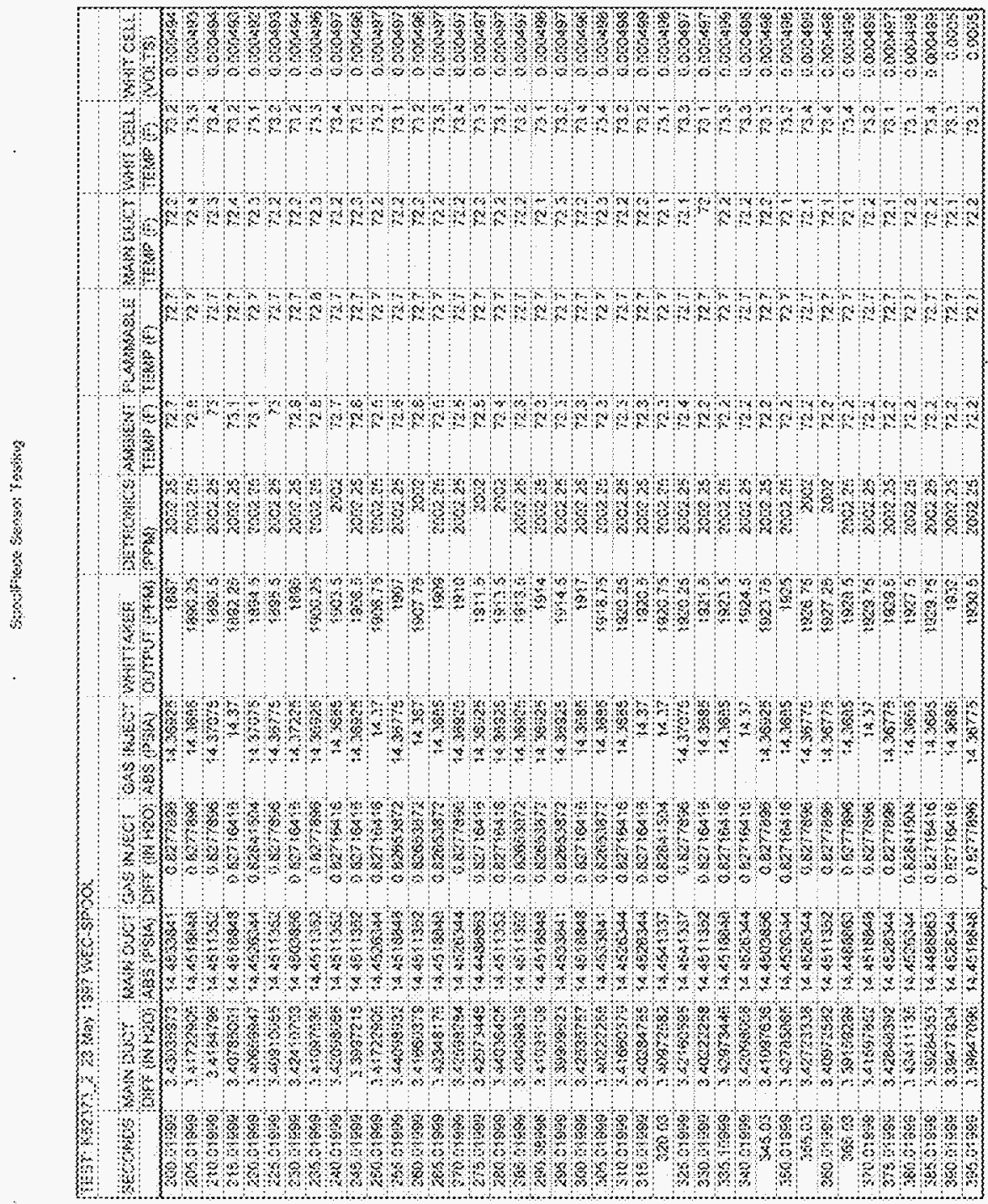




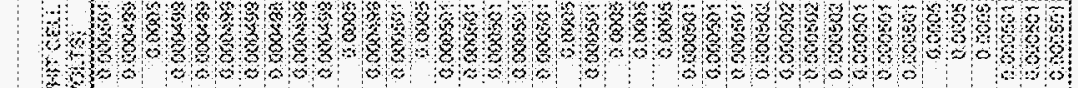 se

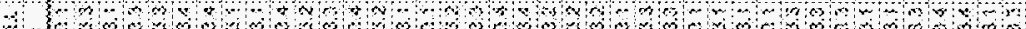

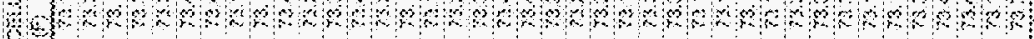
a

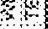

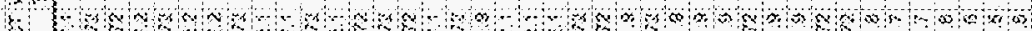

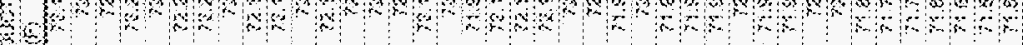
28

\$

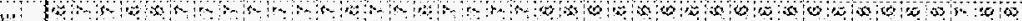
LAW

is

3

mWW Ww

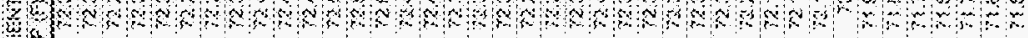

僢

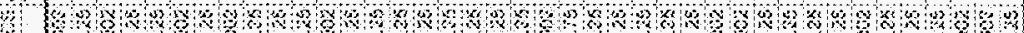

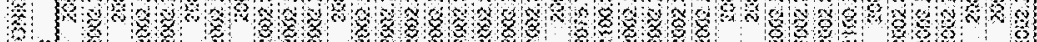

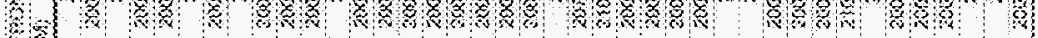
a

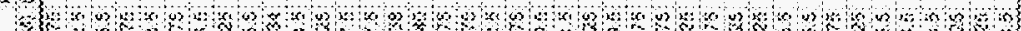

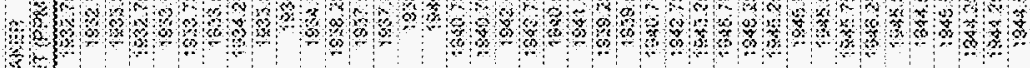

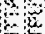

3

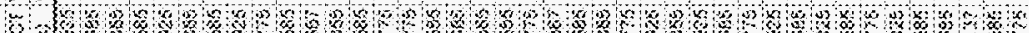

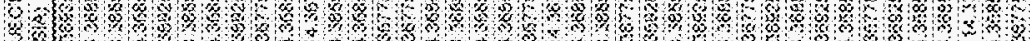

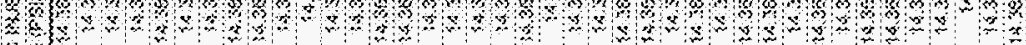
淨

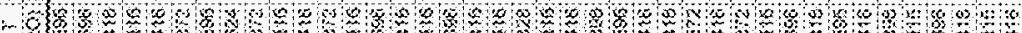

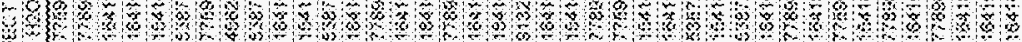

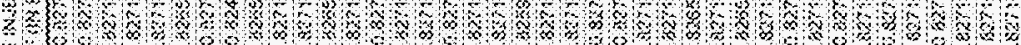

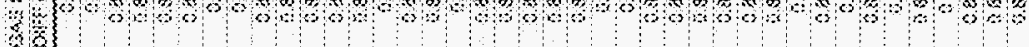

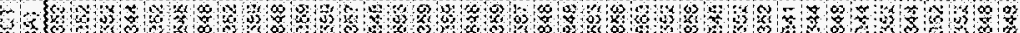

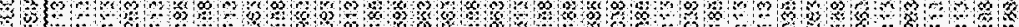

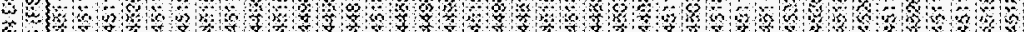

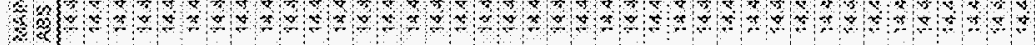

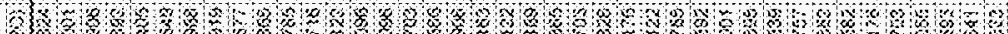

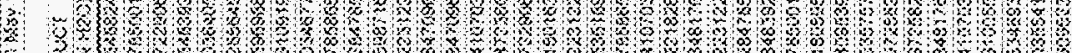

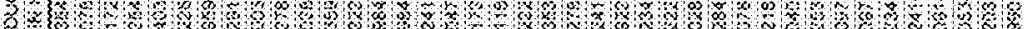

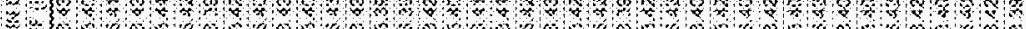

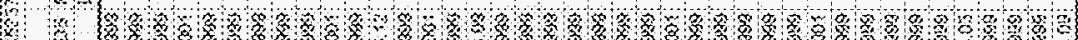

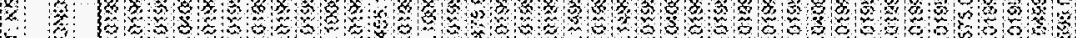




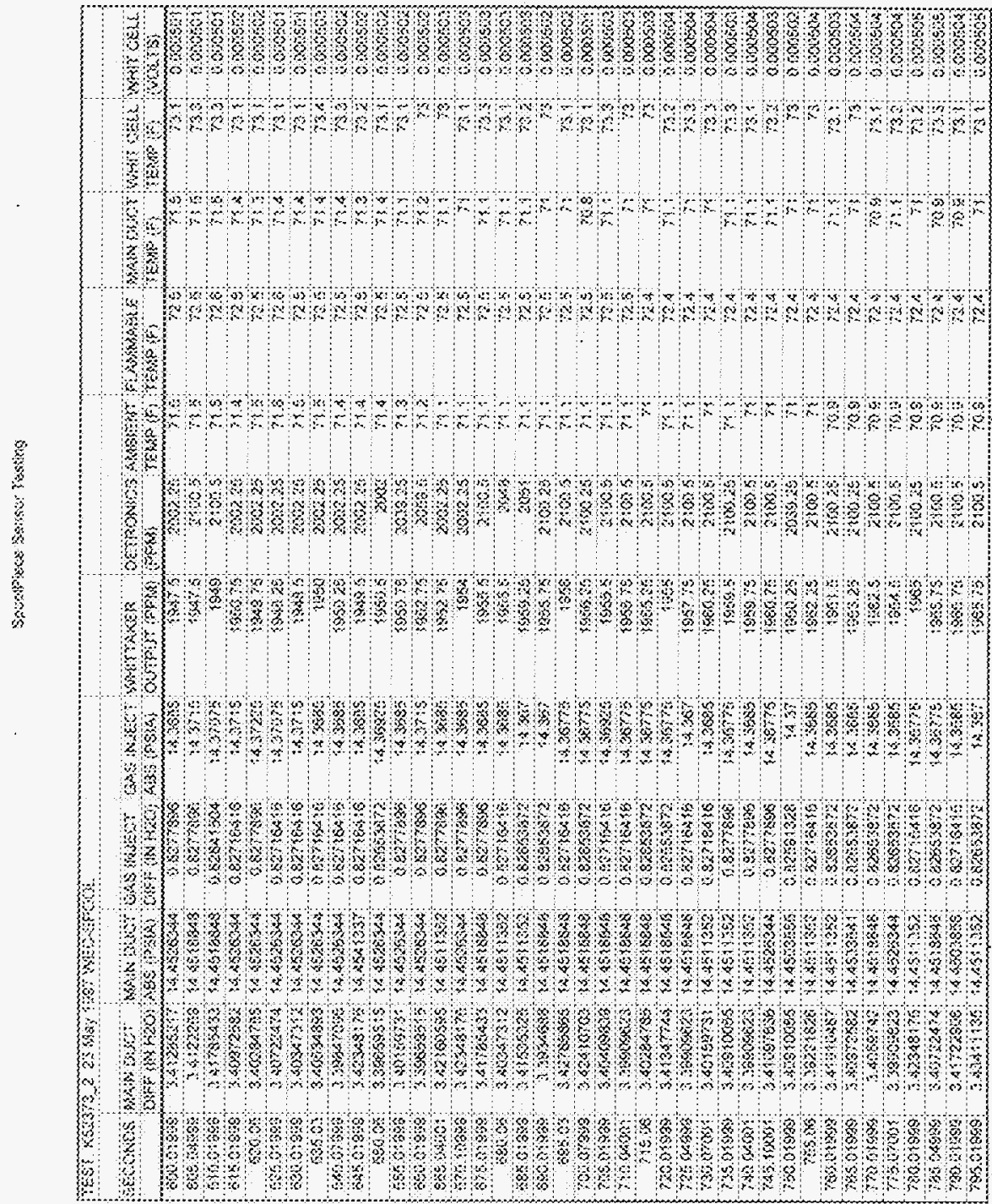




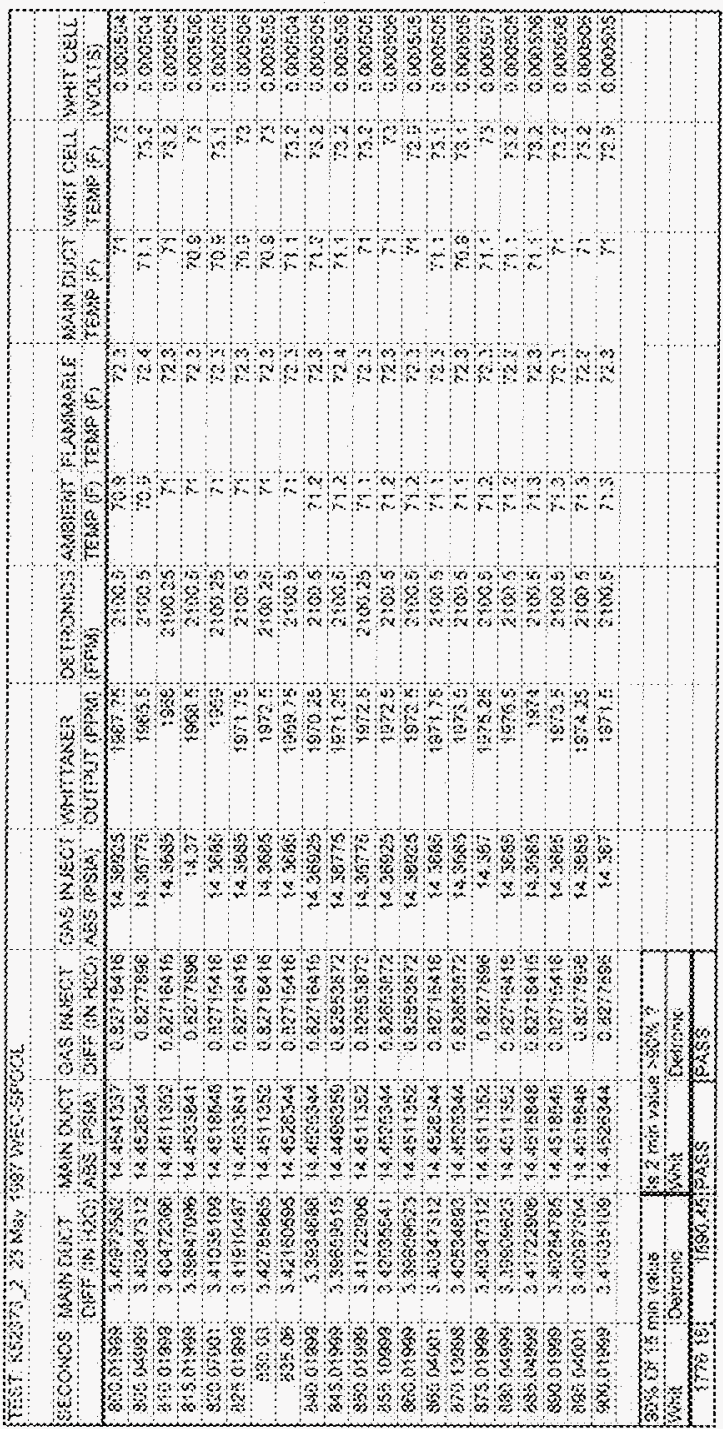




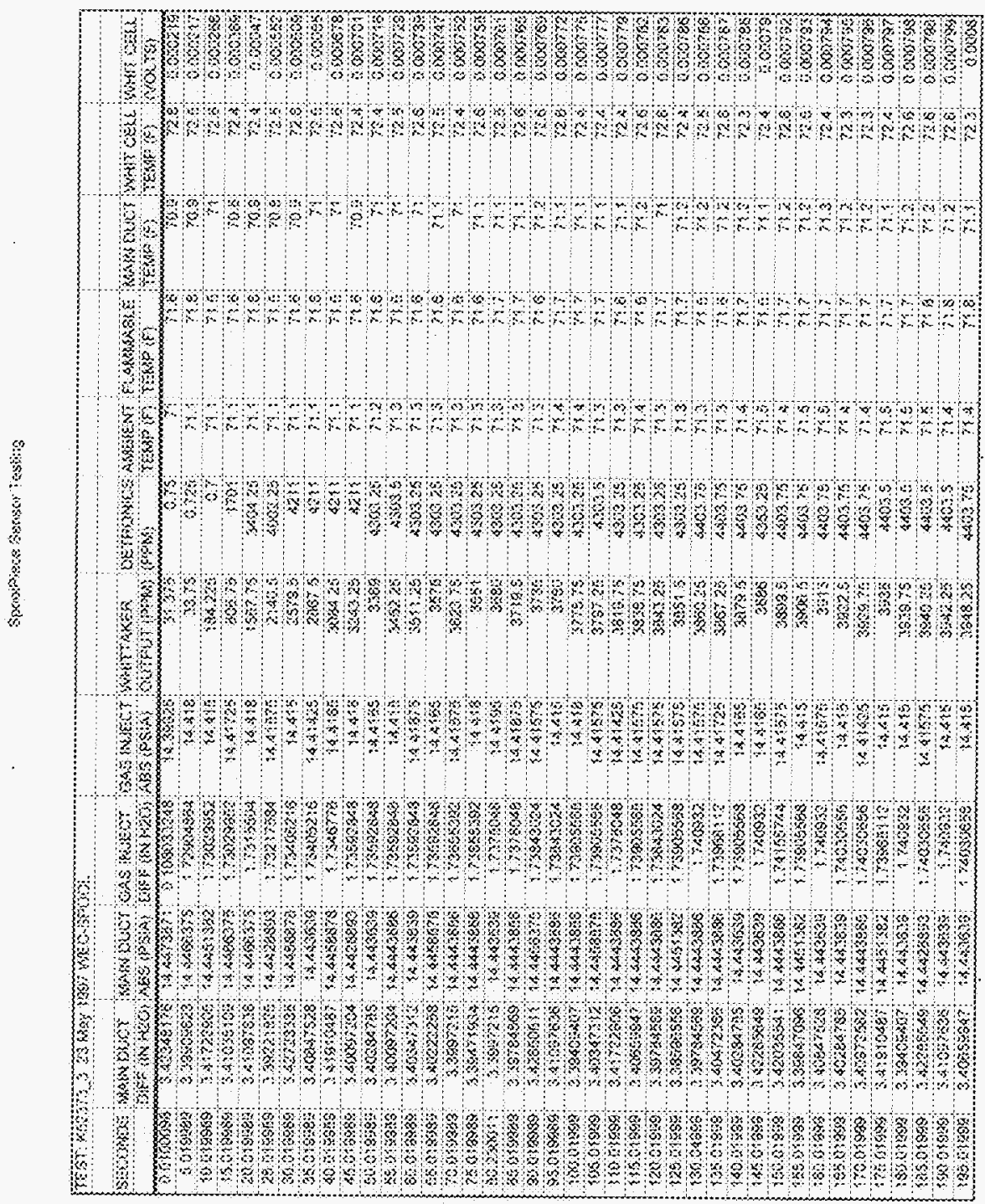




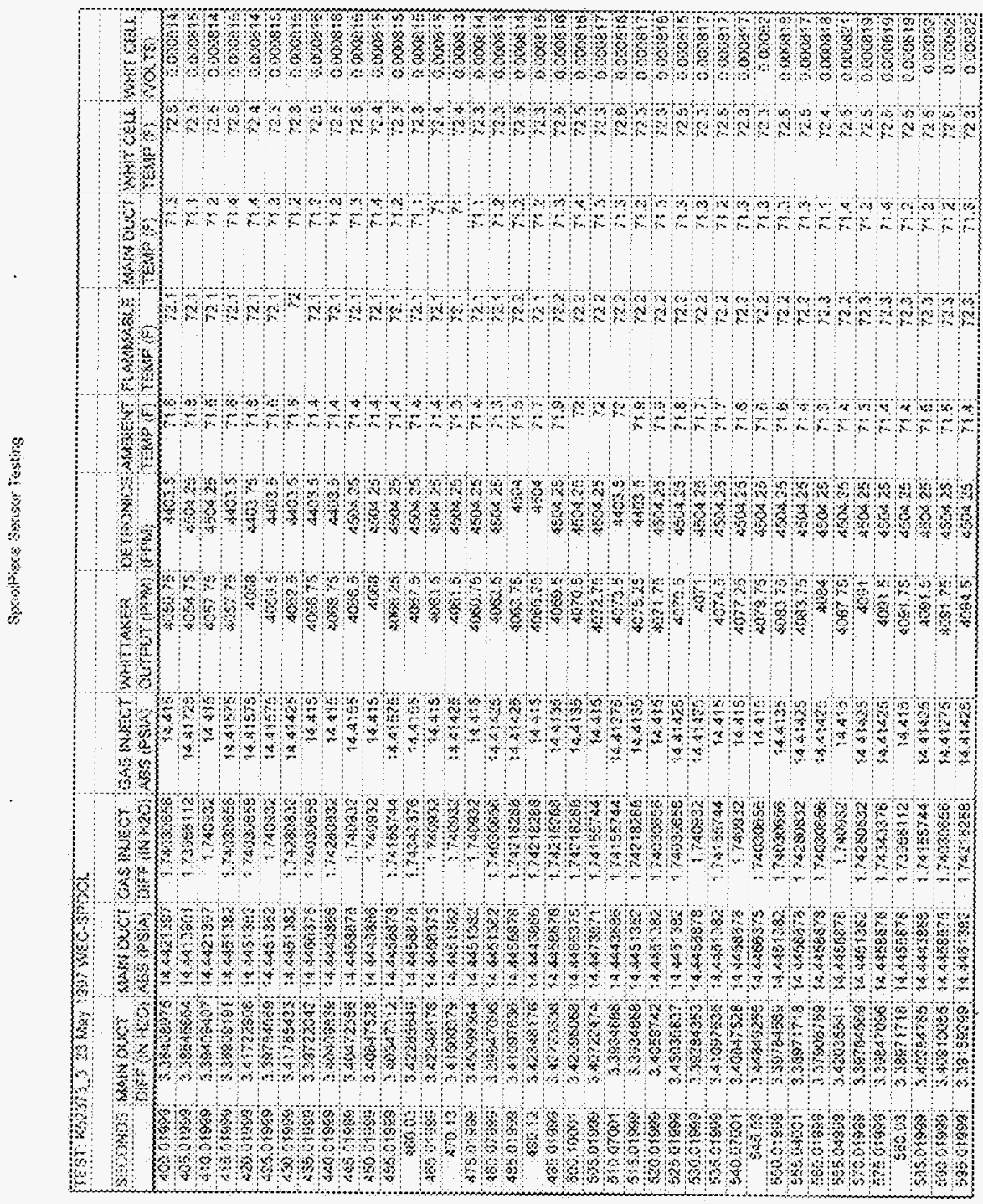




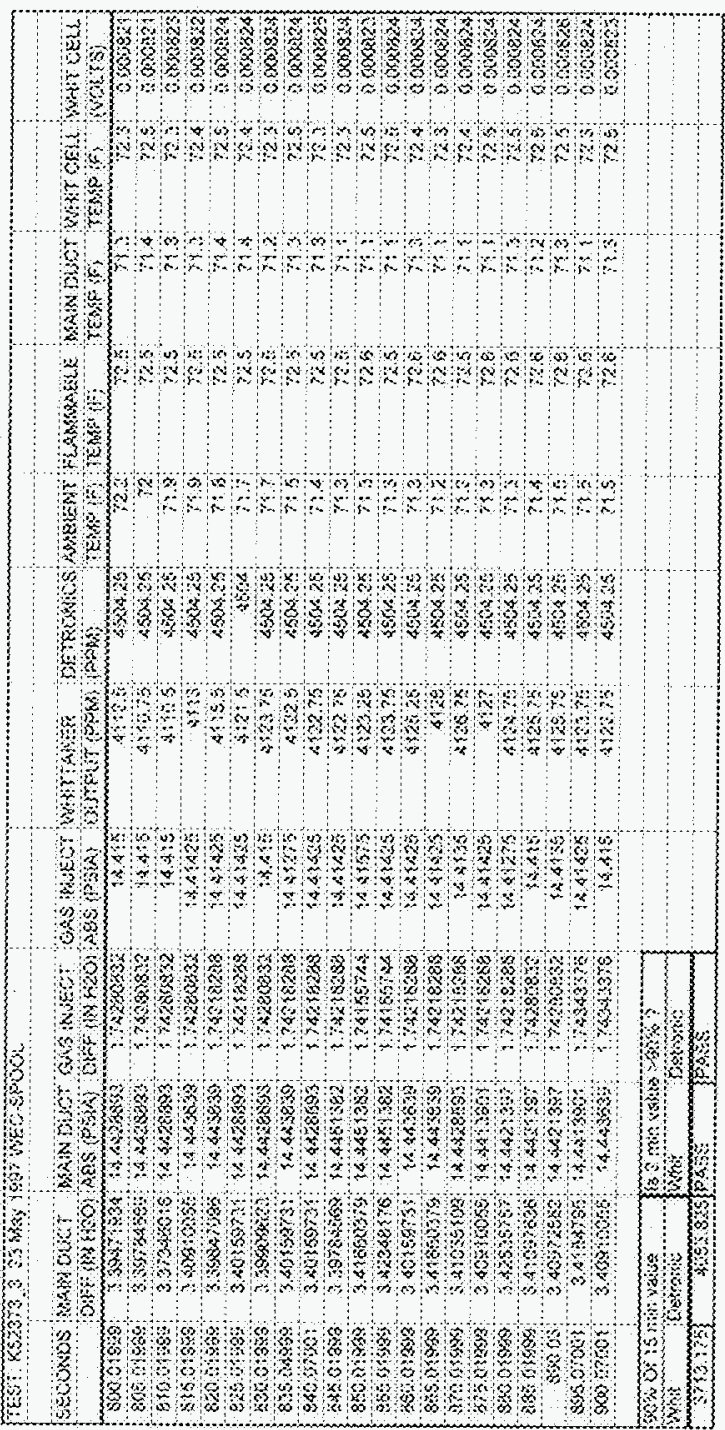




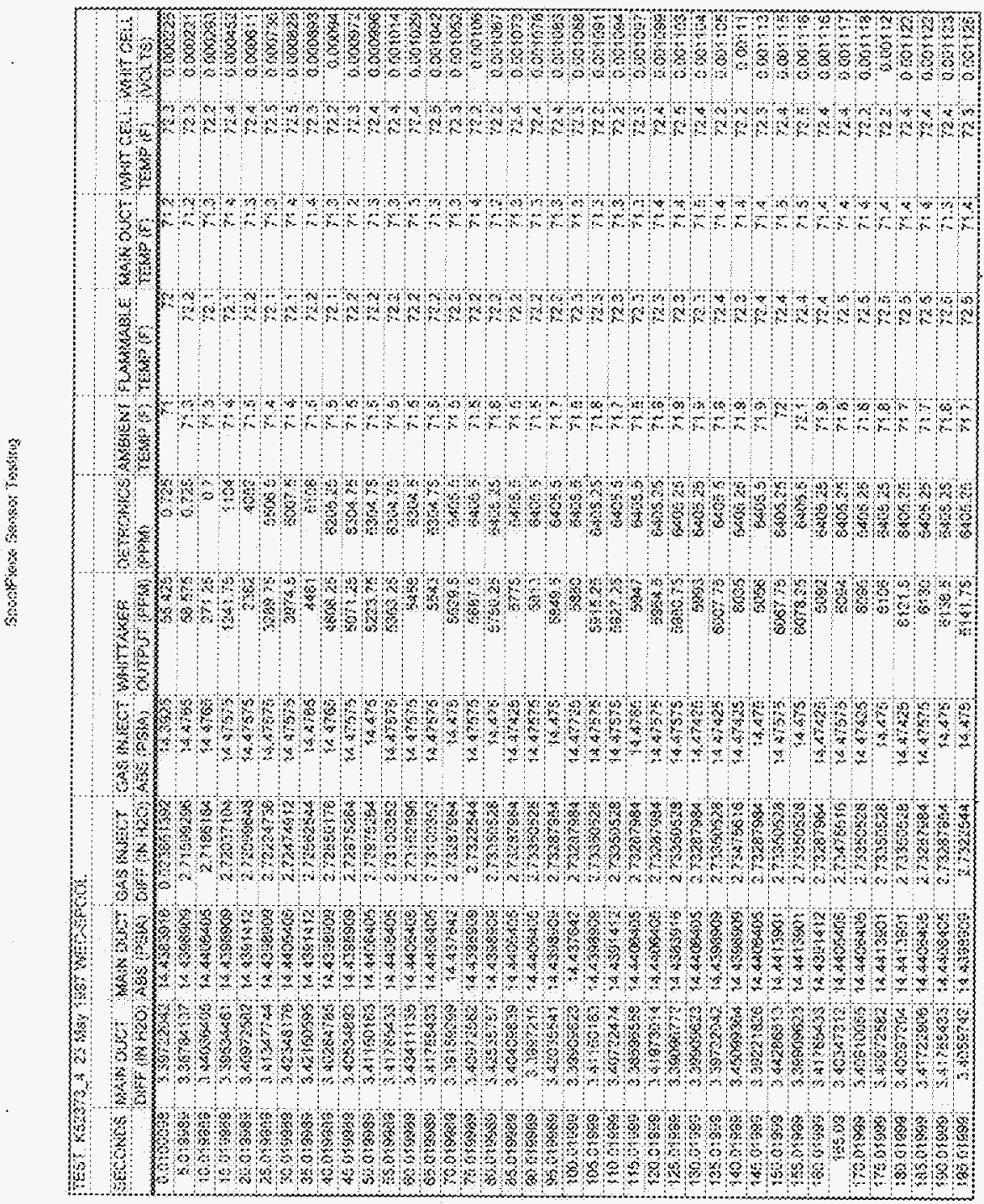




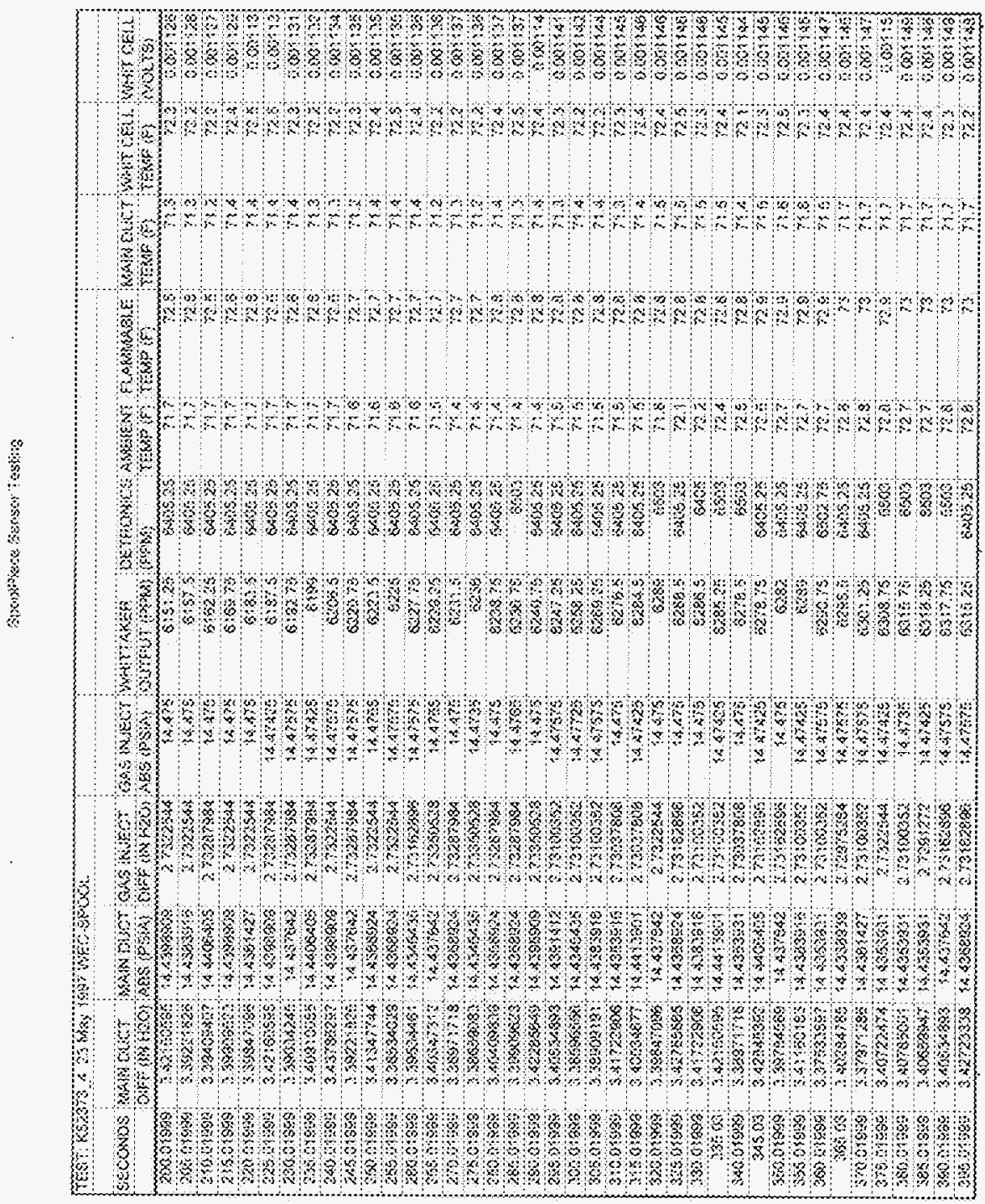




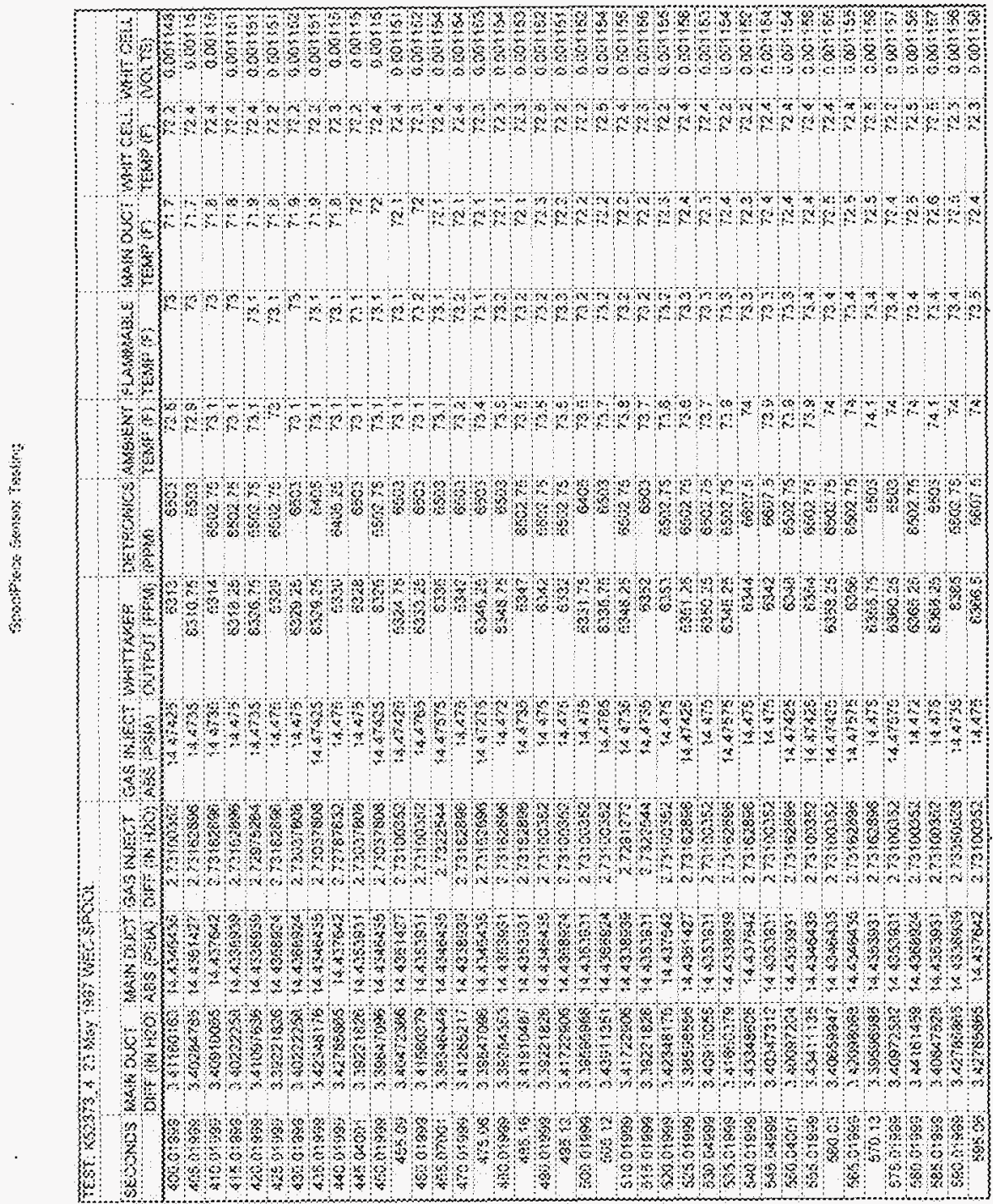




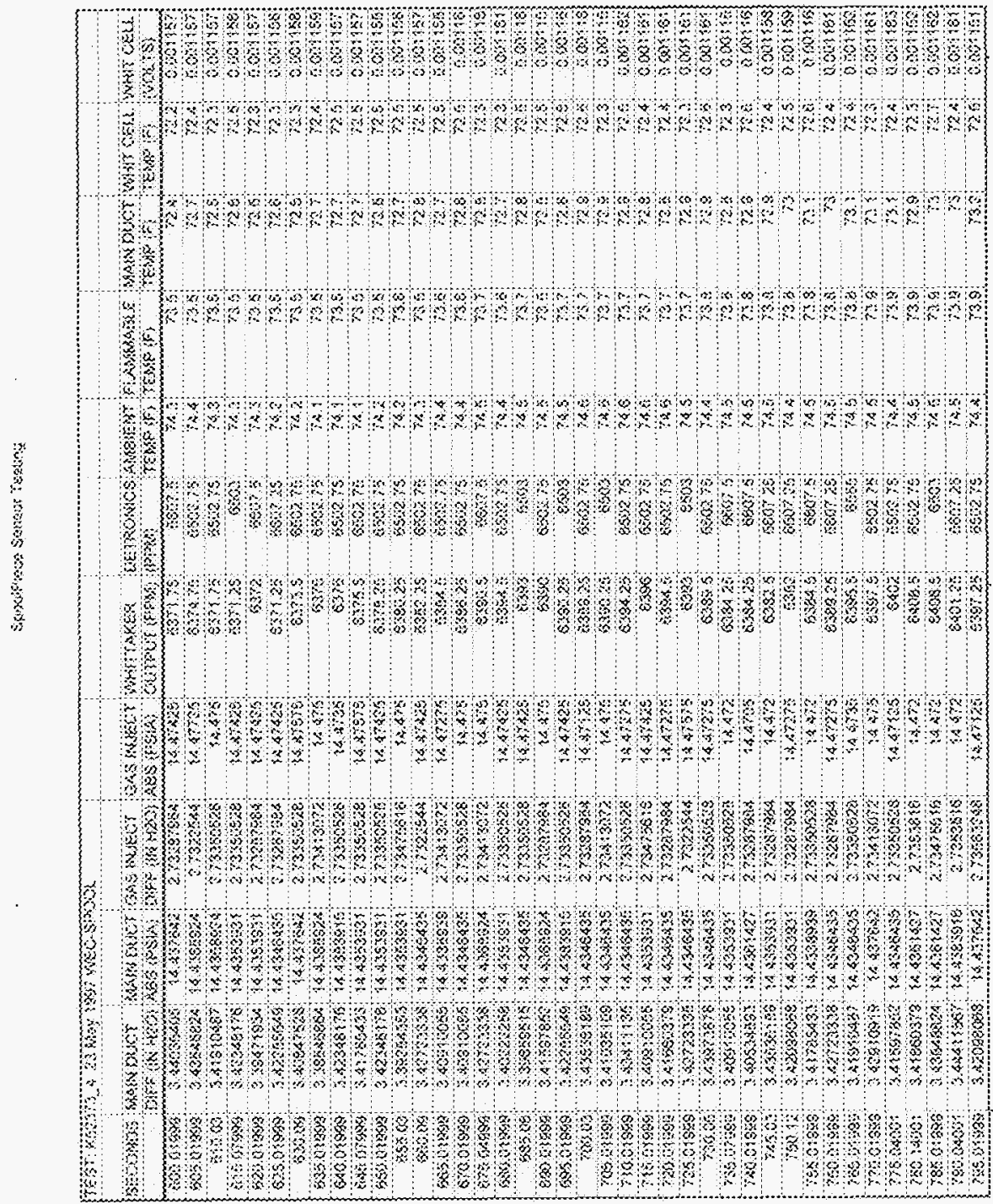




\section{DISTERบTHO SHEK}

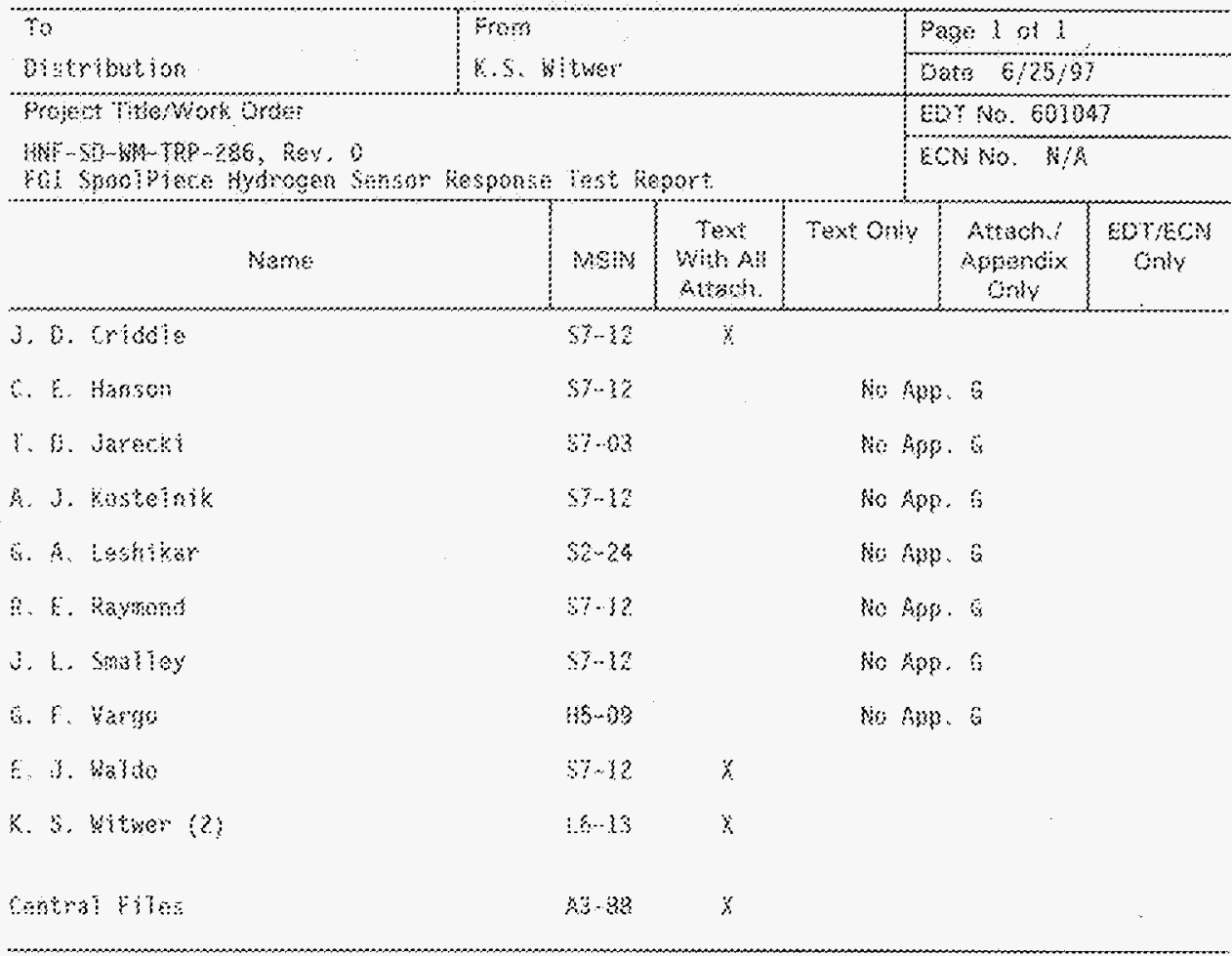

